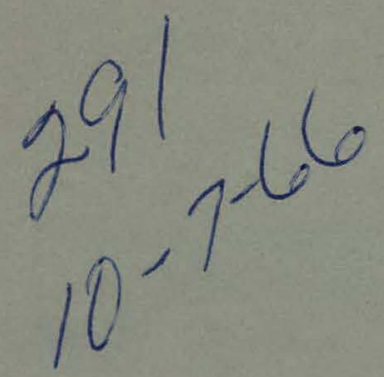

\title{
AN APT POST PROCESSOR \\ FOR POSITIONING MACHINES
}

AUTHORS:

M. G. Buchanan

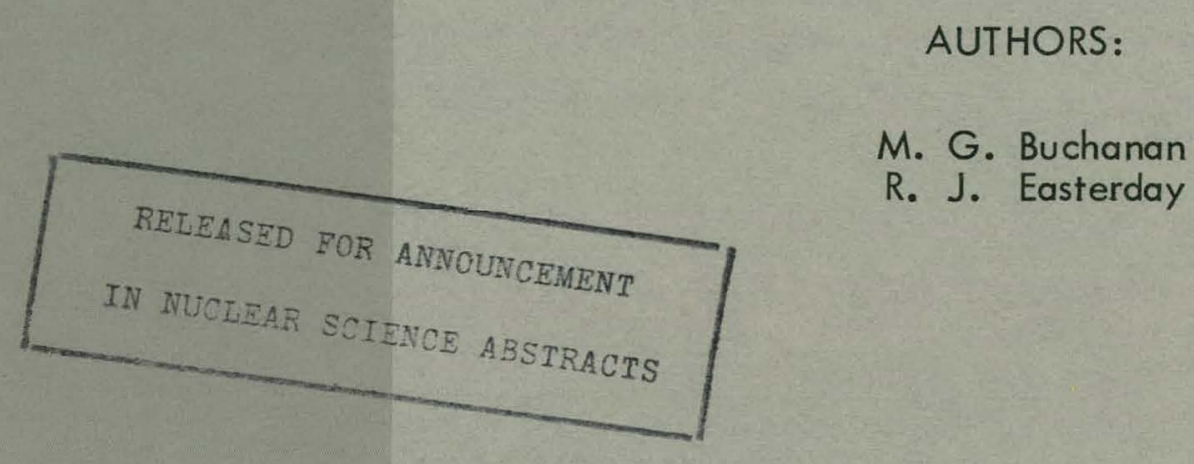

R. J. Easterday

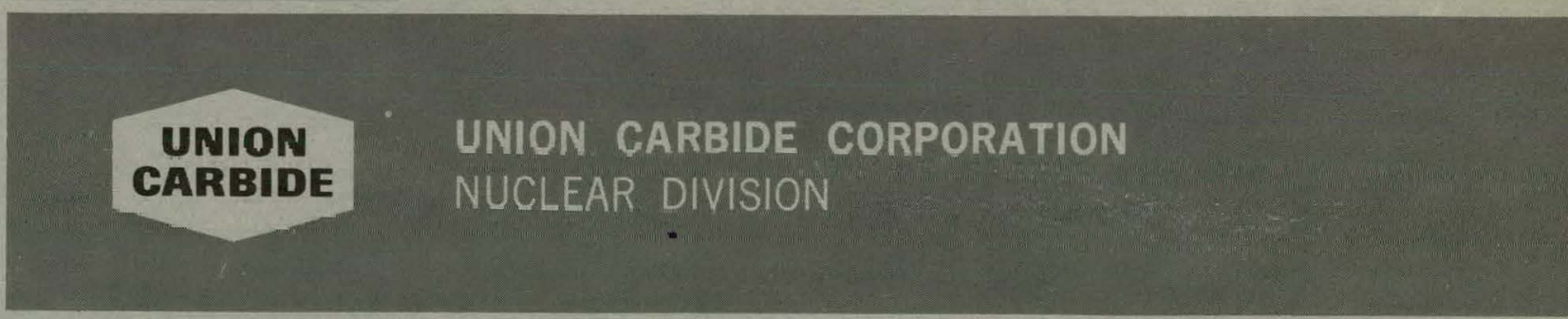

Operating the

OAK RIDGE GASEOUS DIFFUSION PLANT OAK RIDGE $Y$ - 12 PLANT
- OAK RIDGE NATIONAL LABORATORY

- PADUCAH GASEOUS DIFFUSION PLANT 


\section{DISCLAIMER}

This report was prepared as an account of work sponsored by an agency of the United States Government. Neither the United States Government nor any agency Thereof, nor any of their employees, makes any warranty, express or implied, or assumes any legal liability or responsibility for the accuracy, completeness, or usefulness of any information, apparatus, product, or process disclosed, or represents that its use would not infringe privately owned rights. Reference herein to any specific commercial product, process, or service by trade name, trademark, manufacturer, or otherwise does not necessarily constitute or imply its endorsement, recommendation, or favoring by the United States Government or any agency thereof. The views and opinions of authors expressed herein do not necessarily state or reflect those of the United States Government or any agency thereof. 


\section{DISCLAIMER}

Portions of this document may be illegible in electronic image products. Images are produced from the best available original document. 
Printed in USA. Price $\$ 6.00$. Available from the Clearinghouse for Federal Scientific and Technical Information. National Bureau of Standards,

U.S. Department of Commerce, Springfield, Virginia 22151

\section{LEGAL NOTICE}

This report was prepared as an account of Government sponsored work. Neither the United States, nor the Commission, nor any person acting on behalf of the Commission:

A. Makes any warranty or representation, expressed or implied, with respect to the accuracy, completeness, or usefulness of the information contained in this report, or that the use of any information, apparatus, method, or process disclosed in this report may not infringe privately owned rights; or

B. Assumes any liabilities with respect to the use of, or for damages resulting from the use of any information, apparatus, method, or process disclosed in this report.

As used in the above, "person acting on behalf of the Commission" includes any employee or contractor of the Commission, or employee of such contractor, to the extent that such employee or contractor of the Commission, or employee of such contractor prepares, disseminates, or provides access to, any information pursuant to his employment or contract with the Commission, or his employment with such contractor. 


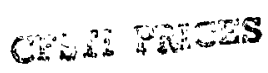

H. . . 6.00 .25

Date of Issue: September 29, 1966

RETEASED FOR ANNOUNCEMENT

Report Number: $\mathrm{K}-1675$

Subject Category: MATHEMATICS

AND

COMPUTERS

(TID-4.500)

IN NUCIEAR SOIENCE ABSTRACTS

AN APT

POST PROCESSOR

FOR

POSITIONING MACHINES

M. G. Buchanan

R. J. Easterday

This report was prepared as an account of Goveroment sponsored work. Neither the United States, nor the Commission, nor any person acting on behalf of the Commission:

A. Makes any warranty or representation, expressed or implied, with respect to the accuracy, completeness, or usefulness of the information contalned in this report, or that the use of any information, apparatus, method, or process disclosed in this report may not infringe privately owned rights; nr

B. Assumes any llabilltues with reapect to the use of, or for damages resultung from the use of any Information, apparatus, method, or process disclosed in this report.

As used in the above. "person acting on behalf of the Commission" includes any employee or contractor of the Commisalon, or employee of such contractor. to the extent that such employee or contractor of the Commission, or employee of such contractor prepares, disseminates, or provides access to, any information pursuant to his employment or contract with the Commission, or his employment with such contractor.

\section{UNION CARBIDE CORPORATION \\ NUCLEAR DIVISION \\ Computer Sciences Center}

Oak Ridge, Tennessee 


\section{THIS PAGE}

WAS INTENTIONALLY

LEFT BLANK 


\begin{abstract}
The APT Post Processor for Positioning Machines is a generalized post processor that produces command tapes for numerically controlled positioning machines. It provides a basic framework that allows easy implementation of new machines.
\end{abstract}


THIS PAGE

WAS INTENTIONALLY

LEFT BLANK 
TABLE OF CONTENTS

Page

1. Description......................... 7

2. Appendix.............................. 11

2.1 Subroutines Affected by Addition of New Machines.... 12

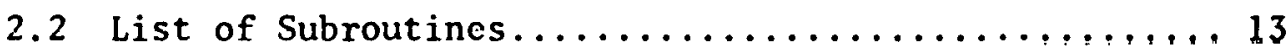

2.3 Descriptions of Subroutines............... 16

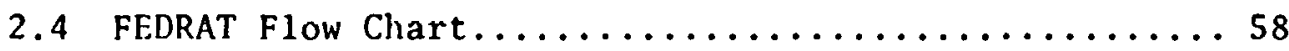

2.5 Description of Tables.................. 59

2.6 Variable Names and Definitions..............66 64

2.7 Post Processor Words.................. 67

2.8 Program Listing of Subroutines............. 70

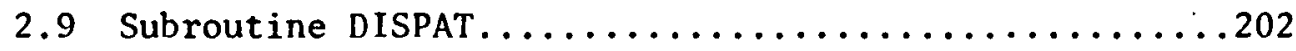


THIS PAGE

\section{WAS INTENTIONALLY LEFT BLANK}


An APT Post Processor for Positioning Machines

1.

Description

The APT Post Processor for Positioning Machines is intended to be a generalized post processor. This post processor provides a basic framework that allows new machines to be added without altering the framework or the machines that have previously been implemented. Features peculiar to a given machine may be taken care of by adding new subroutines and/or by adding new branches to existing subroutines. Section 2.1 of the Appendix makes note of the subroutines generally affected by the addition of new machines.

The flow of the post processor is controlled by subroutine POSITN. POSITN calls subroutine READER which reads the CLTAPE. POSITN then calls other subroutines to process the CLTAPE data. Different branches are made in POSITN according to the type of CLTAPE record that is read. After a record has been processed, READER is called to obtain the next record from the CLTAPE. A cross reference list of the subroutines that comprise the Positioning Post Processor appears in Section 2.2 of the Appendix. Descriptions of all of the subroutines are in Section 2.3 of the Appendix. A flow chart of FEDRAT is included in Section 2.4 because the routine contains a large number of machine dependent branches.

Data to be output is loaded into the PREBU array in floating point representation. Each of the machine's registers is assigned a particular element of PREBU. Prior to output, the floating point number in each element of PREBU is converted to $B C D$ as specified by the corresponding element of the FORMAT array and placed into the appropriate element of BUFFER. If word address is desired, the BCD letters are obtained from the WORDS array, which is ordered like PREBU and FORMAT, and placed in the appropriate elements of BUFFER. A description of the BUFFER, FORMAT, and WORDS arrays is given in Section 2.5 of the Appendix. 
When adding a machine to the Positioning Post Processor, there are two options pertaining to when the output from non-motion CLTAPE records is obtained. The output may either be put out in a block by itself, or it may be held for merging with the next motion block. In the case for which the non-motion output is in a block by itself, PREBU is loaded with the proper values and subroutines CARD and PRINT are called to prepare the punched output and to print the information, respectively. When a value is to be held for merging with the next motion block, the appropriate variable is loaded with the value and the corresponding flag is set to indicate that there is a value being held for later output. A list of variable names with definitions is in Section 2.6 of the Appendix.

When coordinate data is to be put out, POSITN calls subroutine DATA which prepares the calculated points for output as a motion record. To do this, DATA calls subroutine COMPUT which calculates the desired coordinate data and then returns to DATA which then calls subroutine SETUP. SETUP calls STAUxx to load any values being held for output in the next motion block into the appropriate elenents of PREBU. SETUP then loads the coordinate data into the proper PREBU elements. After SETUP completes its function; control is returned to DATA where the coordinate data is rounded to the particular machine accuracy and held as the previous point. DATA then returns to POSITN which calls subroutines CARD and PRINT to put out the information in PREBU. Descriptions of the above routines are included in Section 2.3 of the Appendix.

Tables of the options presently available in the Positioning Post - Processor are available in Section 2.5 of the Appendix. The IDUM array contains the fixed point values and the OPTAB array contains the floating point values. Some elements in each array are used as variable storage locations rather than options. The options and the variables are all initialized to preset values in subroutine INIT. If a particular machine requires that an option have another value, the option may be set to the desired value in MACHxx. 
Included in Section 2.7 of the Appendix is a dictionary of post processor words now available on the Positioning Post Processor. Program listings of all the subroutines are provided in Section 2.8 of the Appendix.

This positioning post processor is designed to operate with the APT III System; however, it is non-standard in that the subroutine XPOST in chain 400 of the standard system must be altered so that the machines called for in the machine statements will be recognized. Subroutine DISPAT in chain 400 must also be altered to place the machine number in MTABLE(6). The MTABLE array is included in the common package of the positioning post processor. If the machine statement format MACHIN/ machine name, machine number is used, the version of subroutine DISPAT listed in Section 2.9 of the Appendix will properly load the machine number in MTABLE(6). 
i

THIS PAGE

\section{WAS INTENTIONALLY \\ LEFT BLANK}


2.

APPENDIX 
2.1 Subroutines Affected by Addition of New Machines

The following subroutines are usually altered when a new machine is added to the Positioning Postprocessor.

Subroutine

INIT

MESSAG

PREINT (LL)

PRINT

PRTON

SETUP

\section{Change}

A call to MACHXX must be added. Machine information for the heading must be added. Tape leader and trailer information must be added. (LI = 0 , leader; LL a 1 , trailer) Machine heading information must be added. Machine heading information must be added for the on-line message.

A call to STAUXX must be added, if registers other than $\mathrm{n}, \mathrm{g}, \mathrm{x}, \mathrm{y}$, and $\mathrm{z}$ are needed.

New subroutines generally needed are:

\section{MACHXX}

STAUXX

NOTE: Other new subroutines may be required by any one machine, but the two mentioned above will be needed for most cases.

The following routines have a branch on the machine number and may be changed to suit the specifications peculiar to the machine. However, there may not be any need to alter these routines, while others not mentioned may need to be altered.

$\begin{array}{ll}\text { AUXFUN } & \text { OPSTOP } \\ \text { CARD } & \text { PREFUN } \\ \text { COOLNT } & \text { REWIND } \\ \text { DISPLY } & \text { SPINDL } \\ \text { END } & \text { SPLCOD } \\ \text { FEDCOD } & \text { STOP } \\ \text { FEDRAT } & \text { TOOLN }\end{array}$

LOADTL 
2.2

I. POSITN (Control Program)

A. AUXFUN

1. CARD

2. PRINT

B. CARD

1. CONBCD

2. PUNCHA

a. PUNCH

b. PUNIDS

3. SEQNO (2)

C. CLRSRF

D. COOLNT

1. CARD

2. COMENT

a. COMTI

3. PRINT

E. DATA

1. COMPUT

a. COMENT

2. SETUP

a. STAUXX

b. PERROR

F. DELAY

1. CARD

2. PRINT

G. DESPAT

1. DISPAT

H. DISPLY

1. CARD

2. COMENT

a. COMPI

3. PRINT

I. END

1. CARD

2. ENDMA4.

a. CONBCD

b. PRINT

c. PUNCHA

3. ENDMA6

a. PUNCHA

4. PRINT

5. PUNCHA

J. FEDRAT

1. $C \dot{A R D}$

2. COMENT

3. FEDCl

a. COMENT

b. FEDCOD

c. PERROR 
K. FINAI

1. DESPAT

2. PREINT

3. PRTON

L. INIT

1. INITI

2. MACHXX

3. PERROR

M. INSERT

1. MESSAG

2. PUNCHA

N. LEADER

1. CONBCD

2. MESSAG

3. PUNCHA

0. LOADTL

1. CARD

2. PRINT

P. MESSAG

Q. OPSTOP

1. CARD

2. PRINT

R. OPTION

S. PDUMP

T. PERROR

1. FINAL

2. PDUMP

U. PREFUIN

1. CARD

2. PRINT

V. PREINT

1. PARNEW

2. PARNOM

3. PUNCHA

4. PUNIDN

5. PUNSEQ

6. SWAP

W. PRINI

1. CONBCD

X. RAPI

Y. READER

Z. REWIND

1. CARD

2. PRINT

AA. ROTABL

1. CARD

2. COMEINI

3. DATA

4. PRINT 
BB. $\quad \operatorname{SEQNO}(\mathrm{N})$

1. COMENT

2. PUNCHA

CC. SPINDL

1. CARD

2. COMENTI

3. PRINT

4. SPINCI

a. COMENT

b. PERROR

c. SPLCOD

DD. STOP

1. CARD

2. PRINT

EE. TOOLN

1. CARD

2. PRINT

FF. TRANS

1. COMENT 
2.3 Descriptions of Subroutines

Subroutine AUXFUN

\section{Purpose}

To output or obtain for later output the auxiliary function number as given by the APT source statement "AUXFUN".

Input

CALL AUXFUN

Output

A block of tape and corresponding listing is produced containing the $\mathrm{m}$-code only, except that the sequence. number, if in the format of the machine, will be included or a parameter and a flag are set for output with next motion.

Method

For machines that output this command with the next motion, the parameter OPTAB (25) is set to the value following the slash in a AUXFUN/_ statement. The flag IDUM (24) is set so that the parameter will be picked up.

For machines that output immediately, the proper PREBU register is loaded and CARD is called to punch the record. PRINT is called to list the record. 
Subroutine CARD

Purpose

To output a block of punched tape

Input

CALI CARD

Output

A block of punched tape is produced.

Method

The buffer area BUFFER is first blanked out, then a representation of punched tape output. is built up. Each register (PREBU) is tested for having a value to be punched. Those registers having no value to be punched will have "BITS" in the appropriate element of PREBU. If the value is not already $B C D, C O N B C D$ is called to convert to $B C D$ per corresponding format in FORMAT table. The BCD representation is then placed in the appropriate location in BUFFER. If word address is desired, the alphabetic label for each register is taken from WORDS and placed in the appropriate location in BUFFER. A branch on machine is made to place tabs in the appropriate places in BUFFER. PUNCHA is called to punch per EIA standard code the BCD information in BUFFER. 
Subroutine CLRSRF

\section{Purpose}

To turn the "CLRSRF" flag on and to store the normal distance to the clearance plane from the origin in OPTAB(27)

Input

\section{CALL CLRSRF}

Output

The normal distance from the origin to the clearance plane is taken from TAPSTO(10) and stored in OPTAB(27). It is then put out in the next motion block.

Method

The "CLRSRF" flag, $\operatorname{IDUM}(34)$, is turned on and the contents of TAPSTO(10) are stored in OPTAB(27). 


\section{Subroutine COMENT}

\section{Purpose}

To write out the information received from COMPl and to increment the line counter for the number of lines output

Input

\section{CALL COMEIT}

Output

The message received from COMPl is printed. 
Subroutine COMPUT

\section{Purpose}

To compute increments of motion

Input

CALL COMPUT

Output

The coordinate points for the next motion block are placed in PRESTX, PRESTY, and PRESTZ.

\section{Method}

Coordinates from the CLTAPE record just read are placed into PRESTX, PRESTY, and PRESTZ. Increments of motion from the previous position are computed for $\mathrm{x}$ and $\mathrm{y}$, and for $\mathrm{z}$ if the machine has three axes. These increments are respectively $\operatorname{OPTAB}(2), \operatorname{OPTAB}(3)$, and $\mathrm{OPTAB}(4)$. If an increment exceeds the limit for a particular machine, a warning message is printed. If the machine has a special code for producing long motions in parts, the exceeding increment is halved and the CLTAPE record indexes are set to pick up the same coordinates again. 
Subroutine COMPI

\section{Purpose}

To provide the error messages required by certain of the subroutines

Input

CALL COMTI.

Outpust.

Subroutine COMENT is provided with the message to be output. 
Subroutine COOLNT

\section{Purpose}

To set up the coolant condition flag as specified by the APT source statement "COOLNT" or to output the appropriate coolant condition code

Input

CALL COOLNT

Output

The flag NCOOL is set indicating that the code for turning coolant on or off is to be punched and the parameter KOOL is set to represent flood (1), mist (2), or off (0) condition; or a block of tape and corresponding listing is produced containing the coolant code only, except that the sequence number, if in the format of the machine, will be included. 
Subroutine DATA

\section{Purpose}

To handle the loading of the registers

Input

CALL DATA

Output

The PREBU array will contain any values to be punched.

$\underline{\text { Method }}$

COMPUT is called to determine the present coordinate values and the increments of motion. SETUP is ral.led to load the coordinates, and any values being held for a motion block, into the PREBU array. Upon return from SETUP, the previous coordinates are rounded to agree with the values put into the punched lape. 


\section{Subroutine DELAY}

\section{Purpose}

To cause the tool to dwell for the number of counts designated in the "DELAY" statement

Input

CALL DELAY

Output

Either a block of tape is punched for each count designated in the "DELAY" statement or the counter on m-codes in hold is incremented, depending on the machine. The DELAY flag is turned on in the latter case.

Method

The number of counts to be held is taken from TAPSTO (4) and stored in the variable "IN" used for the limit in the loop that outputs the m-codes. 
Subroutine DISPLY

\section{Purpose}

To output or obtain for later output the display number as given by the APT source statement "DISPLY"

Input

\section{CALL DISPLY}

Output

A block of punched tape and corresponding listing is produced or parameters and flags are set for output with next motion.

\section{Method}

For machines that output this command with the next motion, parameters are set to the values following the slash in a DISPLY/--statement. Flags are set so that these parameters will be picked up.

For machines that output immediately, the proper registers are loaded and CARD is called to punch the record. PRINT is called to list the record. 
Subroutine END

\section{Purpose}

To produce appropriate output block for the APT source statement "END"

Input

CALL END

Output

Any special end codes will be punched and anything remaining in the punch buffer will be punched.

Method

If special codes are required for an END statement, a call is made to ENDMAx to output them. A call is made to PUNCHA to punch out any information remaining in the punch buffer. 
Subroutine ENDMAx

\section{Purpose}

To produce a block of punched tape containing special end codes appropriate for the machine

Input

CALL ENDMAX

Output

The special end codes will be punched along with any information remaining in the punch buffers.

Method

The required codes are stored in buffer locations and then subroutine PUNCHA is called to punch the information. 
Subroutine FEDC1

\section{Purpose}

To search the feed rate table to find a value corresponding to the specified value from the APT source statement "FEDRAT"

Input

CALL FEDCl

Output

The applicable element number of the feed rate table is placed in NFEEDC.

\section{Method}

The feedrate table (FEDTAB) is searched for the closest value that is not bigger than the specified feed rate value. FEDTAB is arranged as $F(I, J)$ with $J$ being the index of feedrate values and $I$ the index for range. $F(1,1), F(2,1), F(1,2), F(2,2), F(1,3), F(2,3), \ldots$, $F(1, n), F(2, n)$ for example is the order of elements for two ranges and $n$ speeds per range. NFEEDC is set equal to the element number of FEDTAB at which the desired feed rate value is located and a call is made to FEDCOD to obtain the corresponding feed rate code.

\section{Error Checks}

Feed rate table search fails -- Error number 2 
Subroutine FEDCOD

\section{Purpose}

To determine the code corresponding to a value from the feed rate table

Input

CALL FEDCOD. NFEEDC will contain the clcment number of the feed rate table.

Output

Feed rate code will be in NFEEDC.

Method

The feed rate codes for some machines correspond to the value of NFEEDC as determined by FEDCl. For machines where the codes do not correspond, the appropriate code is elther delerinined by a formula or obtained from a table. When a machine having fced rate codes is added, its table of codes or its code determination procedure is added to this routine. 
Subroutine FEDRAT

\section{Purpose}

To obtain values from the APT. source statement "FEDRAT", and output or hold for later output the appropriate feed rate code.

Input

CALL FEDRAT

Output

Feed rate value will be in FEDIPR, range will be in OPTAB(6), and FEDFLG will be set to indicate that a feed rate code is to be picked up, or a block of tape containing the proper feed rate code will be produced along with the corresponding listing.

\section{Method}

If IPR is specified the value of feed rate goes into FEDIPR, if IPM is specified, the value goes into FEDIPM. Any range specified is placed in OPTAB(6) and the flag FEDFLG is set so that the feed rate code will be picked up. The feed rate code is obtained either here, or by a call to FEDCl, and placed in NFEEDC. 
Subroutine FINAL

\section{Purpose}

To punch tape trailer, tally tape footage, and return control to Section 4 control program

Input

CALL FINAL

Output

BCD identification is punched into tape. The length of tape used is printed on line.

Method

PREINT is called to punch trailer. Tape footage is computed and PRTON is called to print on line the tape footage. Control is returned to DESPAT, the main section 4 control program. 
Subroutine INIT

\section{Purpose}

To initialize the program's flags, indexes, parameters, and buffer storages

Input

CALL INIT

Output

The postprocessor program is prepared to process the CLTAPE.

Method

INITl is called to initialize common. Upon return from INITl, the FORMAT and PREBU arrays are filled with bits and the WORDS array is filled with blanks. Feed rate and spindle ranges are initialized to one. The sequence counter increment is set to one. A call is made to the appropriate MACHxx.

\section{Error Checks}

Machine number is greater than 15 - Error number 5 
Subroutine INITI

Purpose

To initialize common storage

Input

CALL INIT],

Output

Common from BITS to ZCAM will be zeroes except that PRESTX, PRESTY, PRESTZ, PREVX, PREVY, and PREVZ will be all bits. 
Subroutine INSERT

\section{Purpose}

To produce a block of punched tape containing the hand-coded block given in the APT source statement INSERT

Input

CALL INSERT

Output

A block of punched tape containing the hand-coded block given in the APT source statement INSERT is produced. 
Subroutine LEADER

\section{Purpose}

To put out the number of inches of leader specified in the "LEADER" statement

Input

CALL LEADER

Oxdput

The number of inches of leader designated in the "LEADER" statement are puriched into the output tape. 
Subroutine LOADTL

\section{Purpose}

To output the codes necessary to load the tool specified by the "LOADTL" statement

Input

CALL LOADTL

Output

The tool length will be in OPTAB(28). A block of tape is punched containing the $\mathrm{m}$-code for a tool change and, if appropriate for the machine involved, the tool and sequence numbers. If required by the machine, a movement to the clearance plane is provided in the block of tape.

\section{Method}

If there are four words per record in the "LOADTL" statement, the fourth word is assumed to be the tool number and the information is output. If there are more than four words in the record associated with the "LOADTL" statement, then the tool length from TAPSTO(10) is stored in OPTAB(28) and the tool number from TAPSTO(8) and the m-code are output. 
Subroutine $\mathrm{MACH} \underline{\mathrm{xx}}$

Purpose

To establish the machine's options, tables, and flags

Input

CALL MACII $\underline{\mathrm{xX}}$

Output

Tables, flags, and options will be loaded with desired values.

Method

The FORMAT, WORDS, SPNTAB, and FEDTAB arrays are loaded with desired information, and OPTAB and IDUM elements are loaded if required. In addition, any standard initial codes may be set up here and punched by calling PUNCHA. 
Subroutine MESSAG

\section{Purpose}

To print the BCD information from the APT source statement "MESSAG"

Input

CALL MESSAG

Output

The message provided in the MESSAG statement is printed. 
Subroutine OPSTOP

Purpose

To provide a planned operator stop

Input

CALL OPSTOP

Output

A block of tape containing the "OPSTOP" m-code is punched. 
Subroutine PERROR

\section{Purpose}

To print out error number when an unrecoverable error'is detected

$\underline{\text { Input }}$

\section{CALL PERROR}

Output

Error number is printed and a core dump'may be given.

Method

The appropriate error number is printed, and if OPTAB. (23) is zero, a core dump is printed. FINAL is then called to terminate the postprocessor. 
Subroutine POSITN

\section{Purpose}

To provide a main control program for the positioning postprocessors

Input

CALL POSITN

Output

A complete CLTAPE is processed and output is prepared ror the printer and punched paper tape.

\section{Method}

The program initializes all necessary parameters and sets up all required tables. The CLTAPE is read by subroutine REALER, and branches are made to major subroutines according to the type of CLTAPE record. Control then returns to READER to obtain another CLTAPE record. For coordinate data records, this subroutine processes one set of coordinates at a time and writes out the results before going on to the next set of coordinates.

\section{Error Checks}

CLTAPE read Failure - Error No. 1

\section{Limitations and Restrictions}

None 


\section{Purpose}

To output, or prepare for later output, the predefined function number as given by the APT source statement "PREFUN"

Input

CALL PREFUN

Output

OPTAB (5) will contain the specified g code and the flag IDUM (16) will be set to pick up the g code at next motion, or a block of punched tape and corresponding listing will be produced containing the g code. 
Subroutine PREINT

\section{Purpose}

PREINT punches a leading or trailing identification on paper tape and also punches an end of block (\$) mark preceding data

Input

CALL PREINT(O) for leader or CALL PRHLNT(1) for trailer.

Output

The routine punches a 90 character I.D. consisting of 24 characters isdentifying the machine and 66 characters as found in PARTNO. The routine also outputs a $(\$)$ punch after the leader.

Method

Each machine has a 24 character BCD identification associated with j.t. These 24 characters and the 66 characters picked up from PARTNO constitute the tape I.D. PARTNO has been stored by the Section 400 "DESPAT" routine in COMMON storage. The PARTIO I.D. is picked up from common and stored along with the machine identification and punched by the routine PARNOM.

\section{Error Check}

None

\section{Limitations}

The Section 400 "DESPAT" routine in use by Union Carbide is nonstandard. The standard Section 400 will not save PARTNO information. 


\section{Subroutine PRINT}

\section{Purpose}

To list the BCD representation of the information punched into the tape

Input

\section{CALL PRINT}

Output

A listing corresponding to the block of information that has been punched into tape is made.

\section{Method}

At the top of each page of output, the machine identification and a heading labeling the registers is written. For each entry, the BCD representation of the punched output is written as located in BUFFER except that decimal points are included in coordinates by an appropriate call to CONBCD which converts the coordinate value in PREBU. Before return, the PREBU array is filled with bits. 


\section{Subroutine PRTON}

\section{Purpose}

To print on line the length of tape punched and the name of the machine being processed.

Input

CALL PRTON

Output

The on-line message is printed. 
Subroutine RAPID

\section{Purpose}

To set the flag that indicates that feed rate is to be at rapid speed

$\underline{\text { Input }}$

CALL RAPID

Output

The rapid flag IDUM (20) will be set to indicate rapid feed rate. 
Subroutine REWIND

\section{Purpose}

To produce the appropriate output block for the APT source statement "REWIND"

Input

CALL REWIND

Output

A block of tape is punched containing only the m-code for a tape rewind except that the sequence number, if in the format of the machine, will be included. A listing is made of the sequence number and rewind code.

\section{Method}

A branch on machine is made to set up the rewind codc in PREBU. CARD is called to output the code. PRINT is called to list the code. 
Subroutine ROTABL

\section{Purpose}

To produce the appropriate output block for the APT source statement "ROTABL"

Input

CALL ROTABL

Output

A block of punched tape and the corresponding listing is produced.

Method

The statement ROTABL/--- is interpreted, and the desired position of the rotary table, if included, or the degrees to rotate if ATANGL is specified is placed into OPTAB (2l). If a value is placed in OPTAB (2l), the flag IDUM (18) is set to pick up rotary table movement. If RPM-is specified, the code for the specified value is determined and placed in OPTAB (24) and the flag IDUM (19) is set to pick up the RPM code. The flag IDUM (15) is set so that COMPUT will be skipped, and DATA is called to load the registers for which there are values being held. CARD is called to output these registers. PRINT is called to list the BCD representation of the punched output. 
Subroutine SEQNO

\section{$\underline{\text { Purpose }}$}

To output the stop rewind code or to setup parameters so that the proper sequence number is produced as requested in the APT source statement "SEQNO"

Input

CALL SEQNO

Output

The stop rewind code is punched or the sequence counter (SEQCTR) and the sequence counter increment (OPTAB(2C)) are set.

\section{Method}

For a SEQNO/START statement PUNCHA is called to punch a stop rewind code. For a SEQNO/k, INCR, $n$ statement the value $n$, if specitied, is placed in OPTAB (22) as the sequence counter increment. If $\mathrm{n}$ is not specified, the value one is assigned. The value $k$ is reduced by OPTAB (22) and placed in SEQCTR so that when incremented by OPTAB (22) prior to next output, the value $\mathrm{k}$ will be the sequence number. 
Subroutine SETUP

\section{Purpose}

To load the registers

Input

CALL SETUP

Output

PREBU registers will contain appropriate values.

\section{Method}

SETUP first loads the sequence counter register, then if the motion is linear, SETUP loads the X, Y, Z registers with their present values. If it is rotary motion, a call to STAUXx is made to load the appropriate registers. In either case, STAUX $\underline{x}$ is called to load appropriate registers with the values being held for a motion record.

\section{Error Checks}

Machine number is greater than 15 - Error number 5 
Subroutine SPINCl

\section{Purpose}

To search the spindle table to find a value corresponding to the specified value from the APT source statement "SPINDL"

Input

CALL SPINC1

Qutput

The applicable element number of the spindle table is placed in NSPIN.

Method

The spindle table (SPNTAB) is searched for the closest value that is not bigger than the specified spindle speed. SPNTAB is arranged as $S(I, J)$ with $J$ being the index of spindle speed values and $I$ the index for range. NSPIN is set equal to the element number of SPNI'AB at which the desired spindle value is located.

\section{Error Checks}

Spindle speed table search fails - Error number 3 
Subroutine SPINDL

\section{Purpose}

To obtain values from the APT source statement "SPINDL"

Input

CALL SPINDL

Output

The spindle speed value will be in RPM, the range will be in $\operatorname{OPTAB}(7)$, and SPIFLG will be set to indicate either that a spindle speed code is to be picked up, or that a block of tape containing the proper spindle speed code will be produced along with corresponding listings.

Method

If RPM is specified, the spindle speed specified goes into RPM. If SFM is specified, RADIUS must also be specified; RPM will be computed using the SFM value and the RADIUS value and will go into RPM. The flag SPIFLG is set so that the spindle speed.code will be picked up. If the machine has multiple ranges, any specified range is placed in OPTAB(7). SPINCl is called to obtain code. 
Subroutine SPLCOD

\section{Purpose}

To determine the code corresponding to a value from the spindle speed table

$\underline{\text { Input }}$

CALL SPLCOD. NSPIN will contain element number of spindle speed table.

Output

NSPIN will contain spindle speed code.

Method

The spindle codes for some machines correspond to the value of NSPIN as determined by SPINCI. For machines where the codes do not correspond, the appropriate code is determined by a formula or obtained from a table. 
Subroutine STAUX $\underline{x}$

\section{Purpose}

To load the registers for auxiliary function values

Input

CALL STAUX $\underline{x}$

Output

The PREBU registers will contain appropriate values. Flags will be cleared.

Method

Flags are tested for those registers applicable to the particular machine for which the routine is written to see. if there are values to be picked up. These values are placed in the appropriate PREBU register and the flags are cleared. 
Subroutine STOP

\section{Purpose}

To produce the appropriate output block and program conditions for the APT source statement "STOP"

Input

CALL STOP

Output

Any special stop codes will be punched and flags for values being held will be cleared.

Method

Any flags set for picking up register values are cleared. If the particular machine requires a special code, it is set up in PREBU, CARD is called to output the code, and PRINT is called to list the code. 
Subroutine TOOLN

\section{Purpose}

To output; or obtain for later output, the" tool number as specified by the APT source statement "TOOLNO"

Input

CALL TOOLN

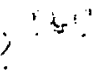

Output

The TOOLNO will be in $\operatorname{OPTAB}(19)$ and the fiag.IDUM(17) will be set.

Method

In a TOOLNO/m, $\mathrm{n}$ statement, if $\mathrm{n}$ is zero or not specified, the value $\mathrm{m}$ is placed in OPTAB(19) and DUUM(17) is set so that the tool number value will be picked up. If $\mathrm{n}$ is non-zero, the tool number value is placed in the appropriate element of PREBU, CARD is called to output the tool number, and PRINT is called to list the tool number. 
Subroutine TRANS

\section{Purpose}

To store the values of $x, y$, and $z$ as given by the APT source statement "TRANS" into the translation vector that is used to translate the coordinate data

Input

CALL TRANS

\section{Output}

TRANSX, TRANSY, and TRANSZ contain the given values of $x, y$, and $z$. The flag NTRANS is set so that the translation will be applied in SETUP. 
2.4

\subsection{SUBROUTINE FEDRAT}

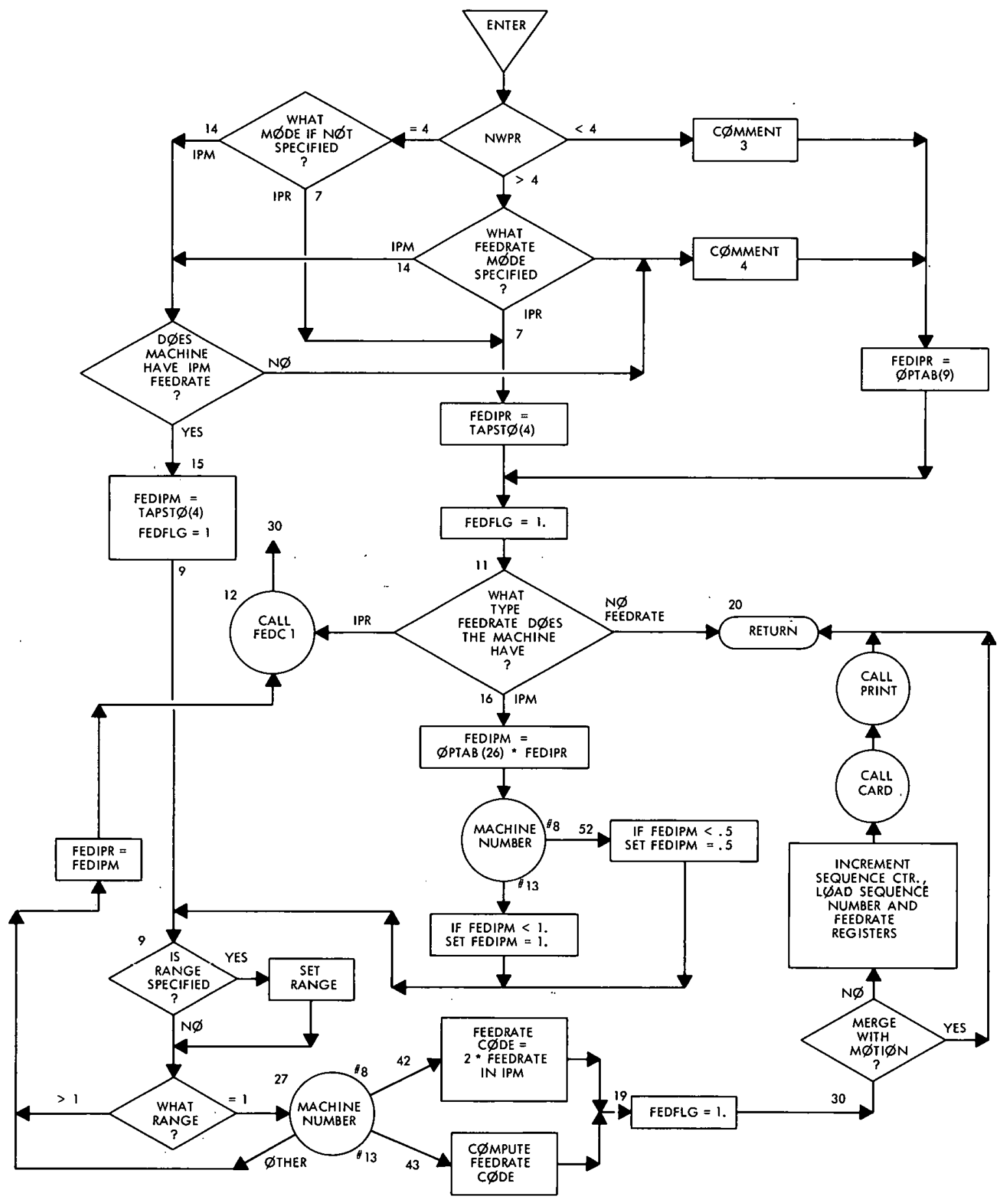




\subsection{Description of Tables}

\section{FORMAT TABLE}

Different machine tools require different output format specifications. Some machines have an $f$ or $s$ register while others do not; some machines require 5 digits for a given register, while others need 6 digits. It is the function of the FORMAT table to handle these variations.

The FORMAT table is an ordered table of 14 locations; each location represents a machine register, and the number assigned to each location specifies the required punch format for values of that register. The assigned numbers are two-digit floating point integers. The first digit of the floating point integer represents the total number of digits to be punched for the specified register, and the second digit indicates the number of digits to the right of the decimal point. If the floating point integer has a minus value, then the sign of the value also will be punched. Thus, the value -54.0 , in the FORMAT table, means that the specified register must have a total of 5 digits punched, four digits being to the right of the decimal point, and one digit to the left of the decimal point, and also that the sign must be punched. The integer 20.0 means that a total of two digits must be punched; two to the left and none to the right of the decimal point, and that there should be no sign.

\section{WORDS TABLE}

$\begin{array}{cc}\text { WORDS } & \text { BCD CONTENTS } \\ \text { 1) } & \mathrm{N} \\ 2) & \mathrm{G} \\ 3) & \mathrm{X} \\ 4) & \mathrm{Y} \\ 5) & \mathrm{Z} \\ 6) & \mathrm{B} \\ 7) & \\ 8) & \mathrm{F} \\ 9) & \mathrm{E} \\ 10) & \mathrm{S} \\ 11) & \mathrm{R} \\ 12) & \mathrm{T} \\ 13) & \\ 14) & \mathrm{M}\end{array}$


BUFFER STORAGE

The BCD images for punched output are set up in common storage in the parameter table BUFFER. The following list shows the format of BUFFER.

BUFFER

$\begin{array}{ll}\text { 1) } & \text { Blank } \\ \text { 2) } & \text { BCD N or blanks } \\ 3 \text { 3) } & \text { Value of N (PREBU(1)) or blanks } \\ 4) & \text { Tab or blanks } \\ 5) & \text { BCD G or blanks } \\ 6) & \text { Value of G (PREBU(2)) or blanks } \\ 7 \text { ) } & \text { Tab or blanks } \\ 8) & \text { BCD X or blanks } \\ 9)-10) & \text { Value of X (PREBU(3)) or blanks } \\ 11) & \text { Tab or blanks } \\ 12) & \text { BCD Y or blanks } \\ 13)-14) \text { Value of Y (PREBU(4)) or blanks } \\ 15) & \text { Tab or blanks } \\ 16) & \text { BCD Z or blanks } \\ 17)-18) & \text { Value of Z (PREBU(5)) or blanks } \\ 19) & \text { Tab or blanks } \\ 20) & \text { BCD B or blanks } \\ 21) & \text { Value of B (PREBU(6)) or blanks } \\ 22) & \text { Tab or blanks } \\ 23) & \text { Blanks } \\ 24) & \text { Blanks } \\ 25) & \text { Blanks } \\ 26) & \text { BCD F or blanks } \\ 27) & \text { Value of F (PREBU(8)) or blanks } \\ 28) & \text { Tab or blanks } \\ 29) & \text { BCD E or blanks } \\ 30) & \text { Value of E (PREBU(9)) or blanks } \\ 31) & \text { Tab or blanks } \\ 32) & \text { BCD S or blanks } \\ 33) & \text { Value of S (PREBU(10)) or blanks } \\ 34) & \text { Tab or blanks } \\ 35) & \text { BCD R or blanks } \\ 36) & \text { Value of R (PREBU(11)) or blanks } \\ 37) & \text { Tab or blanks } \\ 38) & \text { BCD T or blanks } \\ 39) & \text { Value of T (PREBU(12)) or blanks } \\ 40) & \text { Tab or blanks } \\ 41) & \text { Blanks } \\ & \end{array}$


BUFFER

$\begin{array}{ll}\text { 42) } & \text { Blanks } \\ 43) & \text { Blanks } \\ 44) & \text { BCD M or blanks } \\ 45) & \text { Value of M (PREBU(14)) or blanks } \\ \text { 46) } & \text { Carriage return (E.O.B) }\end{array}$

The subroutine CARD takes the values stored in $\operatorname{PREBU}(I)$, where I $=1-14$, and sets up the BUFFER as shown in the previous table with the assistance of the subroutine CONBCD. When a complete line of BUFFER is set up the subroutine PUNCH is called which actually punches the card in EIA standard format. 
OPTION TABLE (Fixed Point)

IDUM
1). 'Is $\mathrm{N} \mathrm{BCD}$ or Float
$(1=\mathrm{BCD} ; \mathrm{O}=$ Float $)$
2) Is G BCD or Float
3) Is $X$ BCD or Float
4) Is $Y$ BCD or Float
5) Is Z BCD or Float
6) Is B BCD or Float
7) Not assigned
8) Is F BCD or Float
9) Is $\mathrm{E} B C D$ or Float
10) Is $S B C D$ or Float
11) Is R BCD or Float
12) Is $T$ BCD or Float
13) Not assigned
14) Is M BCD or Float
15) Do we skip COMPUT
16) PREFUN flag
17) TOOLNO flag
18) ROTABL angle flag
19) ROTABL Speed flag
20) RAPID flag
21) Is Coolant to be by self ( 1 - yes, $0=$ no)
22) Is Feed Rate to be by self ( $I=$ yes, $0=$ no)
23) Is Spindle to be by self ( $1=$ yes, $0=$ no)
24) AUXF'UN Flag
25) Do we delete repeated coordinates ( $0=$ yes, $1=$ no)
26) Flag for testing if first data point
27) Do we delete trailing tabs $(0=$ yes, $1=$ no)
28) Feearrate mode to assume if not specified ( $0=I P R, 1=$ IPM)
29) Seqno flag ( $1=\mathrm{ON} ; 2=\mathrm{OFF} ; 3=\mathrm{ON}$, INCR, $N ; 4=\mathrm{k} ; 5=\mathrm{k}$, INCR, N)
30) Counter on $m$ codes being held for motion block
31) Flag for spindle code being held for motion block
32) Code for spindle condition
33) Delay flag
34) CLRSRF flag 


\section{OPTION TABLE (Floating Point)}

\section{OPTAB}

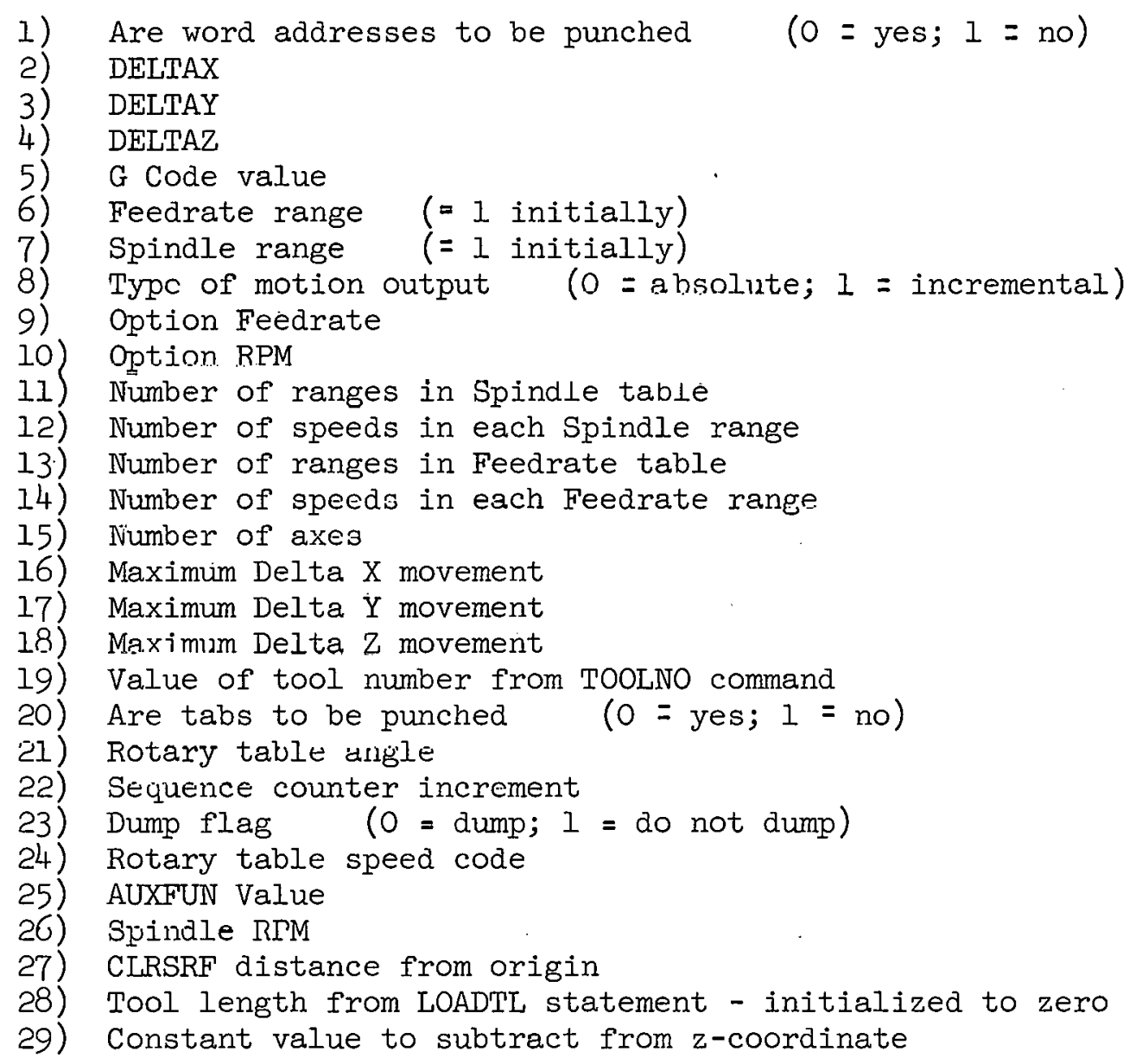




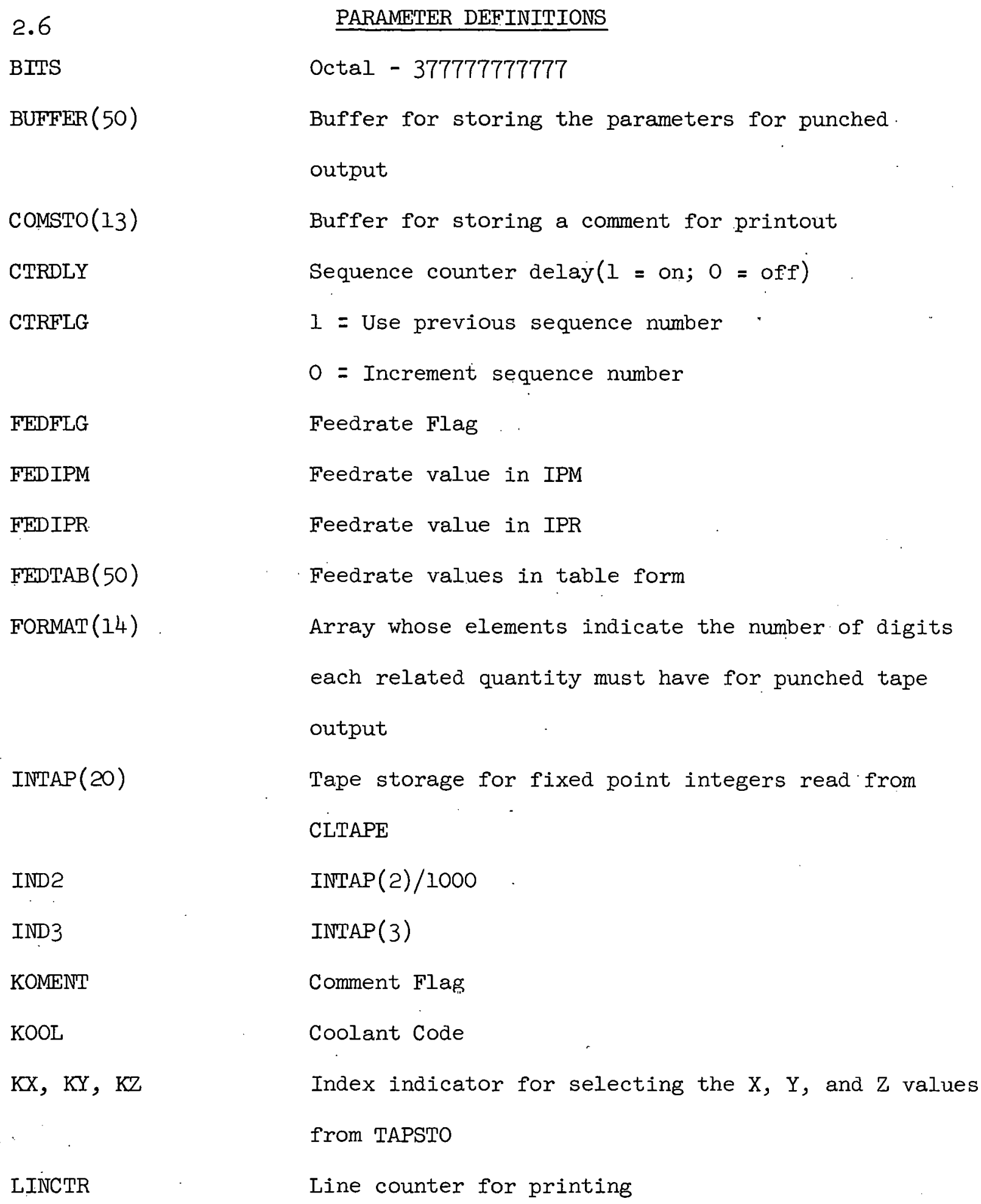




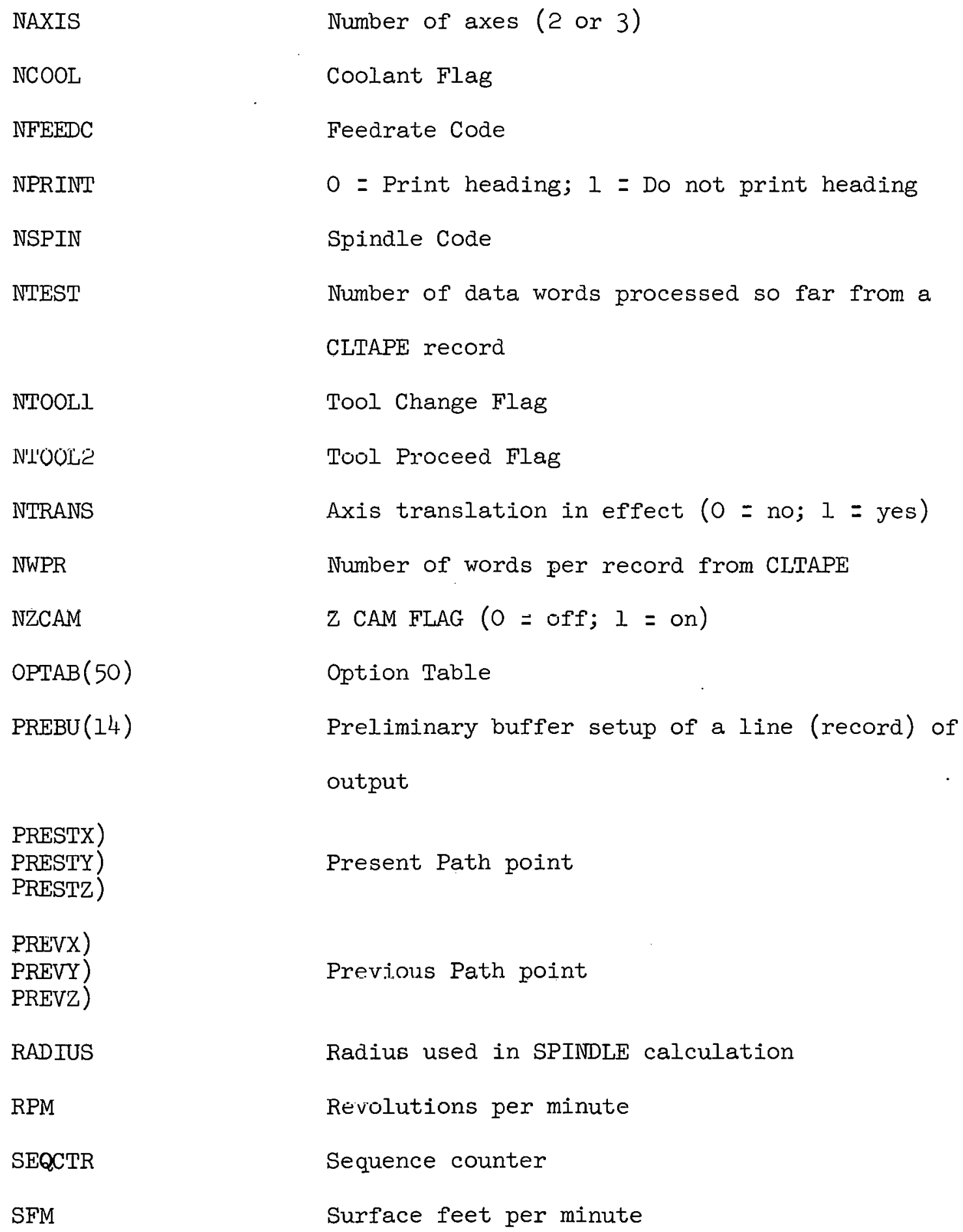


SPIFLG

$\operatorname{SPINAB}(100)$

TAPSTO(250)

TOOLNO

TRANSX )

TRANSY )

TRANSZ )

ZCAM
Spindle Flag

Spindle speed values in table form

Floating point storage of CLTAPE data

Tool number value from DISPLY command

Axis translation values

$\mathrm{Z}$ cam value 
The following auxiliary functions may be used by some or all of the positioning machines.

A. AUXFUN $/ \mathrm{n}$

This instruction outputs the auxiliary function (M Code) specified by $\mathrm{n}$.

B. CLRSRF/(symbolic name of a previously defined surface) CLRSRF/(definition of a surface)

This instruction establishes a clearance for tool retraction.

C. COOLNT/ (ON or OFF)

D. DELAY $/ n$,

$$
/ \mathrm{n}, \mathrm{REV}
$$

$\mathrm{n}$ : Time in seconds of no motion.

$\mathrm{n}$, REV: $\mathrm{n}$ is the number of revolutions of no motion.

E. DISPLY $/ \mathrm{n}, \mathrm{Z}$

This instruction is used to call for a tool change. The " $\mathrm{Z}$ " component of the instruction is used for the Heald only. This indicates depth cam selection with the use of digits 0-9. The " $n$ " can be any number. between 1 and 99 except for the Heald. For the Heald, " $n$ " can be efther the digit 1 or 2. The digit 1 indicates drilling operation; the digit 2 indicates a tapping operalion.

$\begin{array}{llll}\text { F. FEDRAT/f, IPR , RANGE, HIGH } \\ & \text { IPM } & \text { MEDIUM }\end{array}$ LOW

$\mathrm{n}$

$f:$ Desired value of feedrate ${ }^{l}$. IPM): Dimensions of feedrate value; inches per minute or inches per

RANGE: Specifies that the next following word sets the range to

G. LOADTL/n l(LOW), 2(MEDIUM), 3(HIGH), or range number $n$. (modal)

This instruction loads the tool defined into the proper holder; $\mathrm{n}$ may be either a tool number or the symbolic name of a previously defined tool.

I. OPSTOP

This instruction provides a planned operator stop.

I. PREFUN/n

This instruction outputs the predefined function (G code) specified by $n$.

J. RAPD

Specifies rapid feedrate; outputs rapid code in next motion block.

K. REW.LND

This instruction outputs rewind code in block by itself. 
L. ROTABL/m, RPM, $\mathrm{n}$

$\mathrm{m}$ : Desired position of rotary table

RPM: Revolutions per minute

$\mathrm{n}$ : Desired RPM

M. SEQHO/START

This command puts an end-of-record code in the control tape.

$\mathrm{SEQNO} / \mathrm{k}$, INCR, $\mathrm{n}$

The sequence number starts at $\mathrm{k}$ and is incremented by $\mathrm{n}$ for each succeeding block. If $\mathrm{n}$ is not specified, it is assumed to be 1 . $\mathrm{SEQNO} / \mathrm{n}$

The sequence number $\mathrm{n}$ is inserted for the next block only. The succeeding block will be numbered by the previously existing SEQNO option. SEQNO/OPER

The letter"O" code is put in a block by itself.
N. SPINDL/s, RPM, CLW , RANGE, LOW, RADIUS, $r$ SFM, CCLW
n

MEDIUM

$\mathrm{HIGH}$

a: Desired value of spindle speed ${ }^{2}$. RPM) : Dimensions of spindle speed value; revolutions per minute or
surface feet per minute.

RADIUS: The next following word $(r)$ sets the radius for computing RPM.

RANGE: Specifies that the next following word sets the range to 1

0. STOP (LOW), 2(MEDIUM), 3(HIGH), or range number n. (modal)

This instruction clears any code in hold and outputs stop code in block by itself.

P. TOOLNO/m

This instruction generates code for selecting tool number specified by $\mathrm{m}$.

Q. TRANS/ $\mathrm{X}, \mathrm{Y}, \mathrm{Z}$

This instruction can be used to translate cutter coordinates (usually into the first quadrant). " $X$ ". indicates the numerical value of translation along the $X$-axis and " $Y$ " for the $Y$-axis. " $Z$ " indicates the numerical translation along the Z-axis. This instruction is ignored for incremental machines.

R. MACHIN/

1. MAC $\overline{\mathrm{HIN}} /$ GIDLTS, TABLE, 1

2. MACHIN/DVLIEG, JIGMIL, I

3. MACHIN/FOSDIK, JIGBOR, I

4. MACHIN/HEALD, DRILL, 1

5. MACHIN/LAHR, DRILL, 1

6. MACHIN/AMERCN, TABLE, 1

7. MACHIN/KNIGHT, JIGBOR, I

8. MACHIN/BURG, DRILL, 1

9. MACHIN/GIDLWS, DRILL, I 
10. MACHIN/LUCAS, BORMIL, 2

11. MACHIN/GDLWS, DRILL, 2

12. MACHIN/PRATTW, DRILL, 1

13. MACHIN/KRITR, MCHCNT, SERISE

14. MACHIN/GIDLWS, BORMIL, 6INCH

In addition to the above words, the following words are also used:

1. END

2. FINI

3. PARTNO OY TAPENO

1

${ }^{1}$ If $f$ is not exactly available from the feed rate table, the next lowest table value is used. If $f$ is outside the limits of the table for the specified range, the limit nearest $f$ is used.

${ }^{2}$ If $\mathrm{s}$ is not exactly available from the spindle speed table, the next lowest table value is used. If $s$ is outside the limits of the table for the specified range, the limit nearest $s$ is used. 
2.8

*

$\begin{array}{ll}\text { FAP } & \\ \text { PZE } & 0,0,460 \\ \text { TSX } & \text { \$POSITN } \\ \text { MZE } & \text { LAST } \\ \text { BES } & 6940\end{array}$

LAST BES 6940

* END

* count 50

* connector program

ENTRY TAPERD

ENTRY TAPEWT

ENTRY TAPEOP

ENTRY TAPECH

ENTRY SEARCH

ENTRY BUFFTP

ENTRY CARDPN

ENTRY PRCNTL

ENTRY LBSRCH

ENTRY DYNDMP

ENTRY DUMP

ENTRY POUMP

ENTRY (STH)

ENTRY (FIL)

ENTRY (TSH)

ENTRY (RTN)

ENTRY SIN

ENTRY COS

ENTRY SQRT

ENTRY ATAN

ENTRY (STHM)

ENTRY (TSHM)

ENTRY REDPRT

LOCATES SECTION O ROUTINES FOR THIS SECTION

40501100

40501200

40501300

40501400

40501500

40501600

40501700

40501800

40501900

40502000

40502100

40502200

40502300

40502400

40502509

40502600

40502700

40502800

40502900

40523000

40503100

4050320 ?

40503300

40503400

40503500

40503600

$\begin{array}{ll}\text { ENTRY } & \text { CARDRD } \\ \text { ENTRY } & \text { SPECPN }\end{array}$

ENTRY (SPH)

FIRST EQU

100

FIRST + 1

FIRST + 2

FIRST + 3

FIRST +4

FIRST +5

FIRST +6

$4050370 n$

40503702

FORTRAN LOADING POINT

40503800

40503900

ROUTINES VIA THE

SECTION O MAIN PROGRAM

40504000

40504100

40504200

40504300

40504400

40504500

$405046 n$ n

FIRST + 8

FIRST +9

40504700

40504800

40504900

FIRST + 11

40505000

$405051 \mathrm{nn}$

40505200

$4 n 505300$

40505400

40505500

40505600

40505700

40505800

40505900

40506000

(TSHM) TRA FIRST+21

40506100 


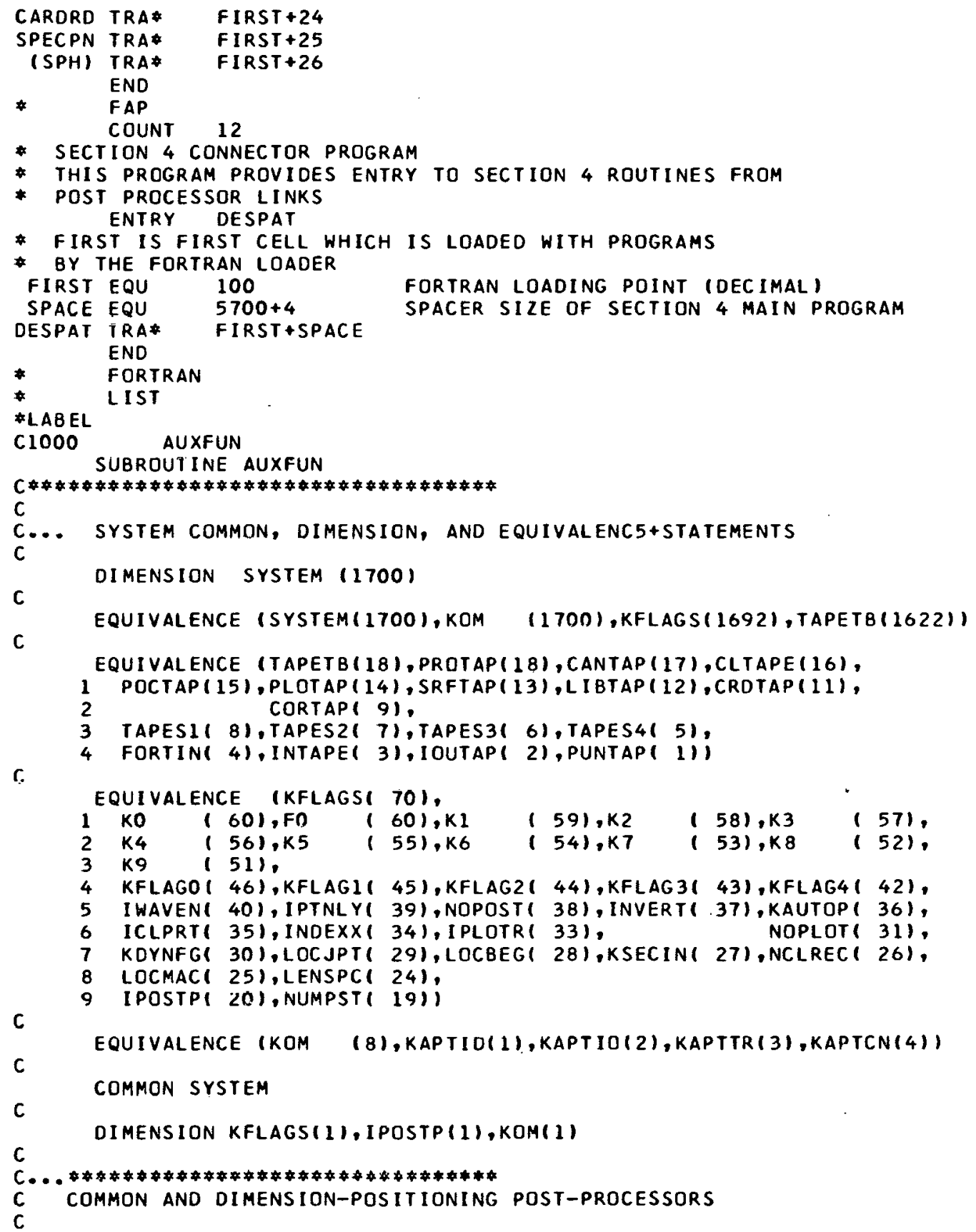

COMMON MACH, NLODP, NI, RIPI, PNOFLG, MTABLE, NID, BITS, BUFFER, COMSTO, 1CTRDLY,CTRFLG, FEDFLG, FEOIPR, FEDTAB, FORMAT, GGL, INTAP, IND2, INO3, 2KOMENT, KOOL, KX, KY, KZ, L INCTR, NAXIS, NCOOL, NFEEDC, NPRINT, 3NODAT, NSPIN, NTEST, NTOOL 1, NTOOL 2 , NTRANS, NWPR, NZCAM, OPTAB,

40506200

40506300

40506400

40506600 40506700 40506800 40506970 40507000 40507300 40507400 4050750 n 40507600 40507700 40506400 


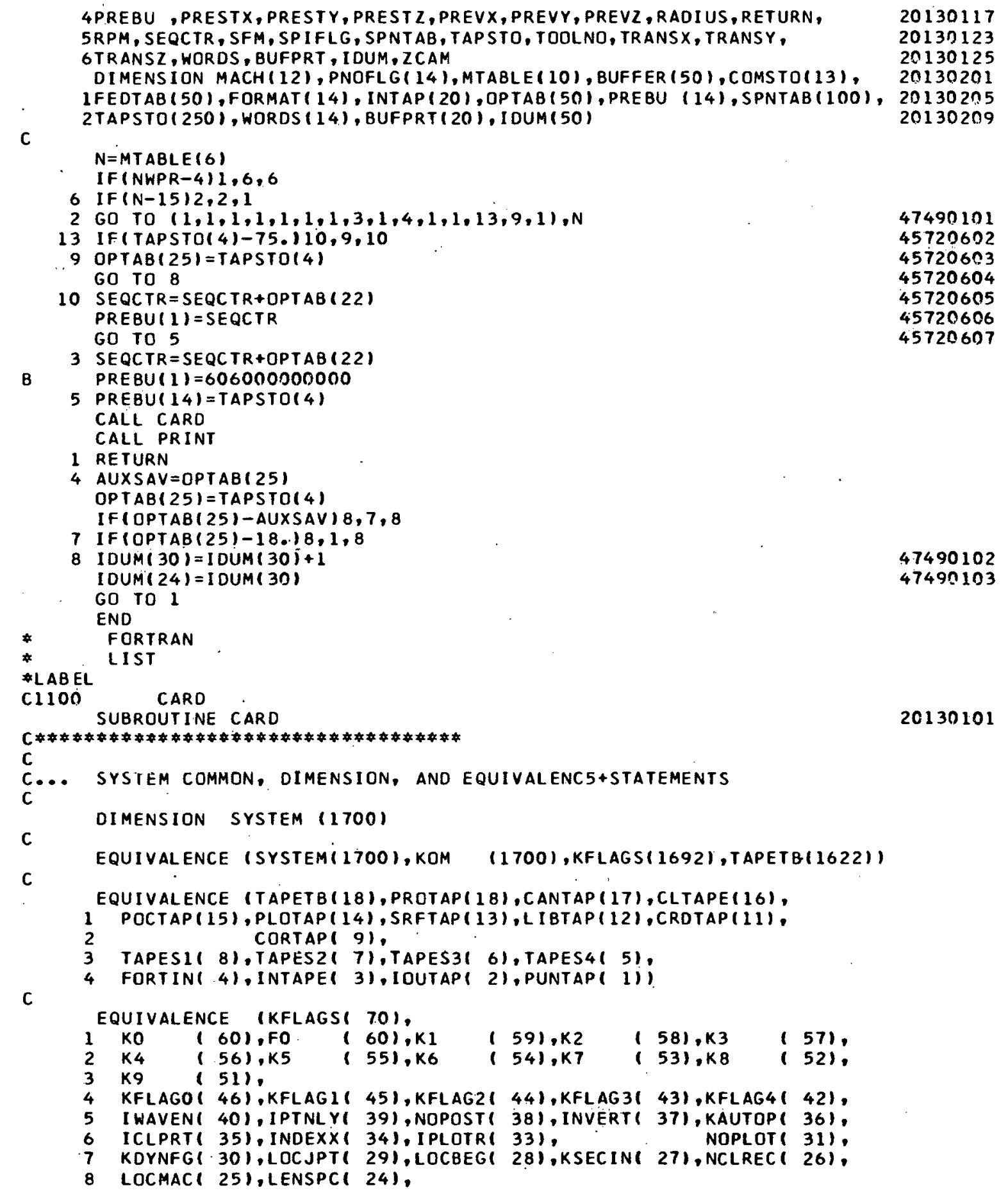


C

9 IPOSTP( 20), NUMPST( 19))

C

EQUIVALENCE IKOM

(8), KAPTID(1), KAPTIO(2), KAPTTR(3), KAPTCN(4))

C COMMON SYSTEM

C DIMENSION KFLAGS(1), IPOSTP(1),KOM(I)

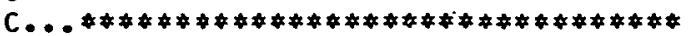

C COMMON AND DIMENSION-POSITIONING POST-PROCESSORS

C

COMMON MACH,NLDOP, NLOOPI, PNOFLG, MTABLE,NID,BITS, BUFFER, COMSTO, 1CTRDL $Y, C T R E L G, F E O F L G, F E D I P R, F E D T A B, F O R M A T, G G L$, INTAP, IND2, IND3, 2KOMENT, KOOL, KX,KY,KL, LINCTR, NAXIS, NCOOL, NFEEDC, NPRINT, 3NODAT, NSPIN, NTEST, NTOOL 1 , NTOOL2, NTRANS, NHPR, NZCAM, OPTAB, 4PREBU, PRESTX, PRESTY, PRESTZ, PREVX,PREVY, PREVZ, RADIUS, RETURN, 5RPM, SEQCTR, SFM, SPIFLG, SPNTAB, TAPSTO, TOOLNO, TRANSX, TRANSY, 6TRANSZ, HORDS, BUFPRT, I DUM, ZCAM

DIMENSION MACH(12), PNOFLG(14),MTABLE( 10), BUFFER(50),COMSTO(13), IFEDTAB ( 50$)$, FORMAT ( 14), INTAP (20), OPTAB (50), PREBU (14), SPNTAB (100), 2TAPSTO $(250)$, WOROS $(14)$, BUFPRT $(20), I D U M(50)$

20130101 20130105 20130111 20130113 20130117 20130123 20130125 20130201 20130205 20130209

C
B
B $B B=606060606060$
BT $=546060606060$ IF (OPT $\triangle B(20)) 18,19,18$

$18 \mathrm{BT}=\mathrm{BB}$

$19 \mathrm{NT}=\mathrm{MTABLE}(6)$ DO $65 \quad J=1,50$

65 BUFFER $(\mathrm{J})=\mathrm{BB}$ DO $50 \quad M M=1,14$ IF (PREBU(MA)-BITS) $35,50,35$

35 IF (FORMAT (MM)-BITS) $25,17,25$

25 AA $=$ FORMAT $(M M) / 10.0$

$N 2=A A+.001$

$B=N 2$

$E=A B S F(F O R M A T(M M)-(B * 10.0))$

$\mathrm{N} 1=\mathrm{E}+.001$

$17 \mathrm{GO} \mathrm{TO}(1,2,3,4,5,6,7,8,9,10,11,12,13,14), \mathrm{MM}$

$1 \mathrm{~N} 3=3$ GO TO 15

$2 N 3=6$ GO TO 15

3 N3 $=9$ GO TO 15

4 N3 $=13$ GO TO 15

$5 \quad N 3=17$ GO TO 15

$6 \quad N 3=21$ GO TO 15

7 N3 $=24$ GO TO 15

$8 \quad N 3=27$ GO TO 15

$9 \quad N 3=30$ GO TO 15

$10 \quad N 3=33$ GO TO 15

11 N $3=36$ 
GO TO 15

12 N3 $=39$

GO TO 15

13 N3 $=42$

GO TO 15

$14 N 3=45$

$15 \operatorname{IF}(\operatorname{IDUM}(M M)-1) 16,40,16$

40 BUFFER $(N 3)=$ PREBU $(M M)$

GO VO 50

16 CALL CONBCD(PREBU(MM), BUFFER (N3), N2,N1)

IF (OPTAB (1)-1.0)20,50,20

20 BUFFER $(N 3-1)=$ WORDS $(M M)$

50 CONT INUE

45 IFINT-15) $46,46,54$

46 GO TO $(51,53,52,51,52,55,55,56,57,58,59,70,80,67,55), N T$

80 . BUFFER $(19)=B T$ GO TO 52

70 BUFFER $(7)=B$ T BUFFER $(11)=B$ T GO TO 55

59 BUFFER (3) $=B B$ GO TO 62

53 BUFFER $(2)=B B$ GO TO 52

67 BUFFER $(23)=B T$ GO TO 68

58 BUFFER $(37)=B T$ BUFFER $(22)=B T$

68 BUFFER $(19)=B T$ BUFFER (31):=BT BUFFER $(37)=B T$

56 BUFFER $(40)=B T$ GO TO 51

57 BUFFER $(31)=B T$ GO TO 51

51 BUFFER (7) = BT

52 BUFFER(4) =BT BUFFER $(28)=B T$

$62 B U F F E R(1)=B T$ BUFFER $(15)=B$ T I F (NT-9) 61, 55,61

61 BUFFER (34)=BT

B 55 BUFFER $(46)=536060606060$ IFI IDUM ( 27$) 160,71,60$

71 DO $72 \quad I=2,14$ $K=16-I$ IFIPREBU(K)-BITS160,100,60

100 GO TO $154,73,74,75,76,77,78,79,81,82,83,84,85,861,1$

73 N3 $=43$ GO TO 72

74 N3 $=40$ GO TO 72

75 N3 $=37$ GO TO 72

76 N $3=34$ GO TO 72

77 N3 $=31$ GO TO 72

$78 \quad$ N $3=28$ 


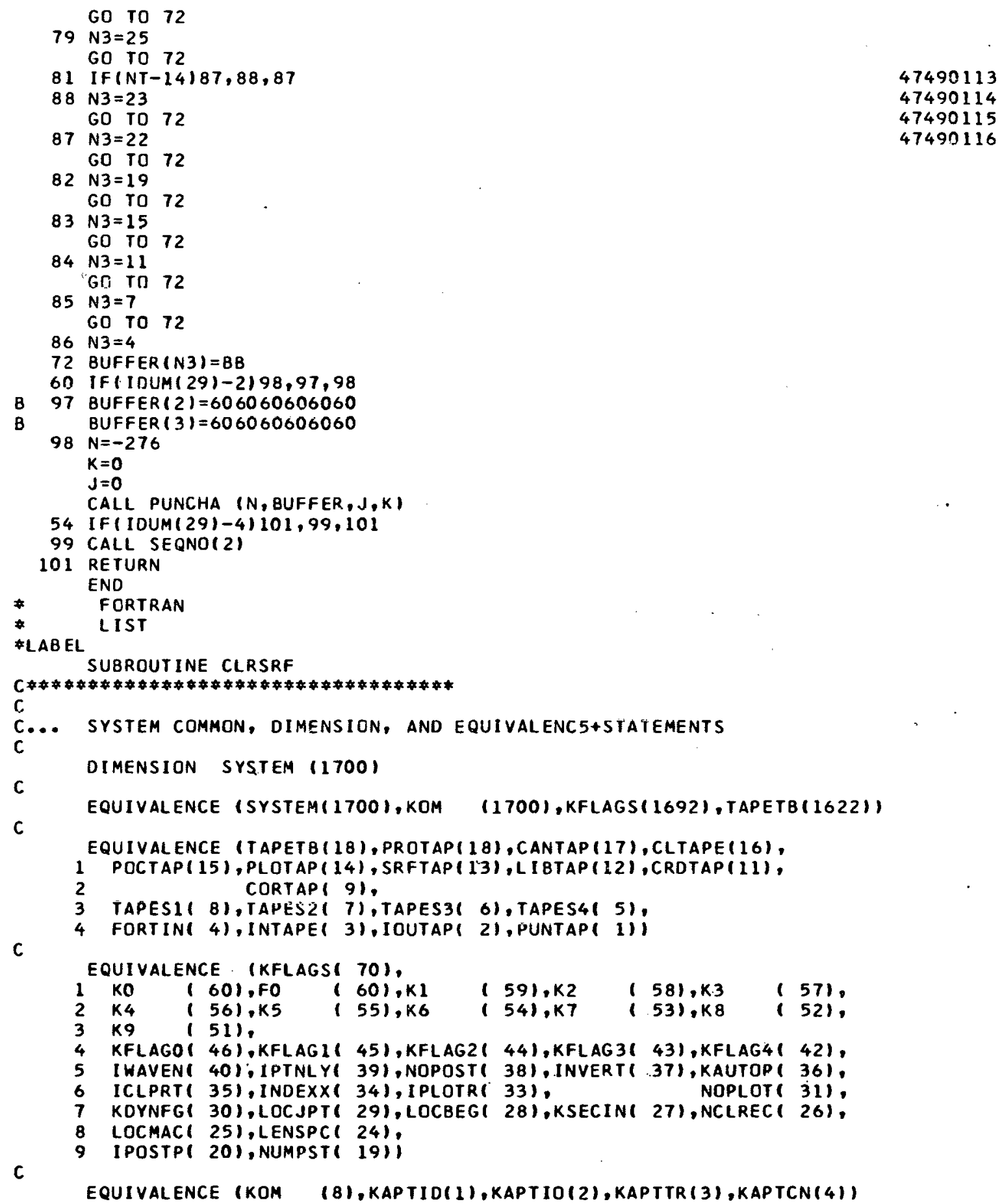


c

C

COMMON SYSTEM

C

OIMENSION KFLAGS(1), IPOSTP(1),KOM(1)

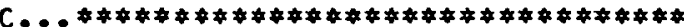

C COMMON AND DIMENSION-POSITIONING POST-PROCESSORS

C

COMMON MACH,NLOOP, NLOOPI, PNOFLG, MTABLE,NID,BITS,BUFFER,COMSTO, ICTRDLY, CTRFLG, FEDFLG, FEDIPR, FEDTAB, FORMAT, GGL, INTAP, IND2, IND3, 20130101 $2 K O M E N T, K O O L, K X, K Y, K Z, L I N C T R, N A X I S, N C O O L, N F E E D C$, NPRINT, 3NODAT, NSPIN,NTEST, NTOOL 1 , NTOOL2, NTRANS, NWPR, NZCAM, OPTAB, 20130105 4PREBU, PRESTX, PRESTY, PRESTZ, PREVX, PREVY, PREVZ, RADIUS, RETURN 5RPM, SEQCTR, SFM, SPIFLG, SPNTAB, TAPSTO, TOOLNO, TRANSX, TRANSY, 6TRANSL, WORDS, BUFPRT, IDUM, ZCAM

DIMENSION MACH(12), PNOFLG(14), MTABLE(10), BUFFER $(50), C 0 M S T O(13)$, 20130111 20130113 20130117 20130123 20130125 IFEDTAB ( 50 ), FORMAT (14), INTAP (20), DPTAB (50),PREBU (14), SPNTAB(100). 20130205 2TAPSTO(250), WORDS( 14$),$ BUFPRT (20), IDUM(50) $\operatorname{IDUM}(34)=1$ OPTAB $(27)=$ TAPSTO $(10)$ RETURN

END

* Fortran

* LIST

\% ABEL

C1200 COMENT

SUBROUTINE COMENT

20130101

C

C... SYSTEM COMMON, DIMENSION, AND EQUIVALENC5+STATEMENTS

C DIMENSION SYSTEM $(1700)$

EQUIVALENCE (SYSTEM(1700),KOM (1700),KFLAGS(1692),TAPETB(1622)

C

EQUIVALENCE (TAPETB(18), PROTAP(18),CANTAP (17), CLTAPE 16$),$ 1 POCTAP (15),PLOTAP (14), SRFTAP(13),LIBTAP(12),CRDTAP 111 ,

2 CORTAP( 9).

3 TAPES1 8 ), TAPES2 ( 7), TAPES3( 6),TAPES4! 5),

C

FORTIN( 4), INTAPE( 3), IOUTAP( 2), PUNTAP( 1)

EQUIVALENCE. (KFLAGS(-70),

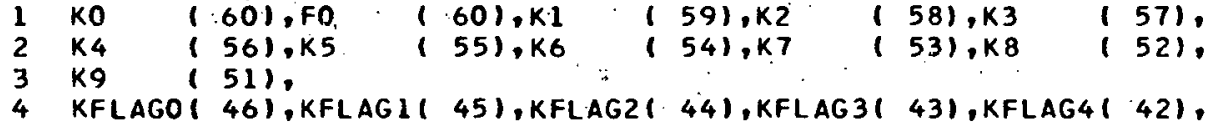

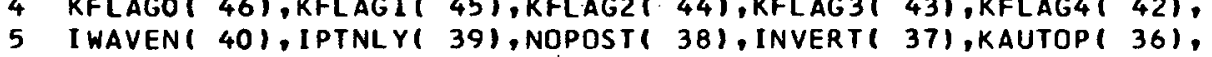
6 ICLPRT( 35), INDEXX( 34), IPLOTR( 33), NOPLOT(.31),
7 KOYNFG( 301 , LOCJPT( 29), LOCBEG( 28),KSECIN( 27), NCLREC( 26$),$
8 LOCMACI 25), LENSPC( 24$)$,
9 IPOSTPI 20I. NUMPST 191$)$

C

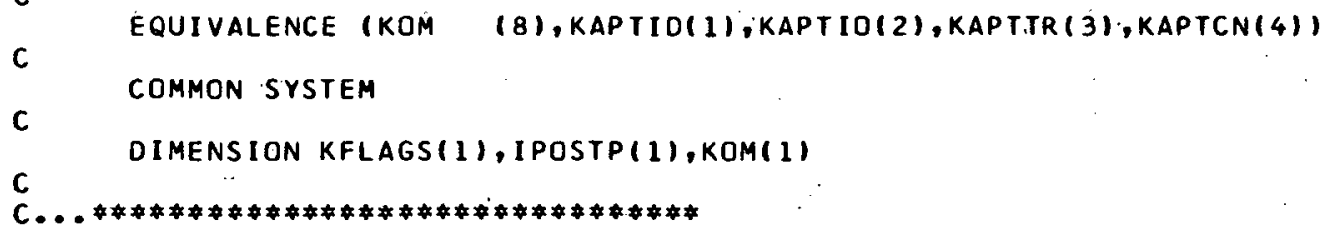


C COMMON ANO DIMENSION-POSITIONING POST-PROCESSORS

C

COMMON MACH, NLOOP, NLOOP 1, PNOFLG,MTABLE,NID,BITS,BUFFER,COMSTO, 20130101 1CTRDLY, CTRFLG, FEDFLG, FEDIPR, FEDTAB, FORMAT, GGL, INTAP, IND2, IND3, 20130105 2KOMENT, KOOL, KX,KY, KZ, LINCTR, NAXIS, NCOOL, NFEEDC, NPRINT, 3NODAT, NSPIN, NTEST, NTOOL I, NTOOL 2, NTRANS, NWPR, NZCAM, OPTAB, 4PREBU ,PRESTX, PRESTY, PRESTZ, PREVX, PREVY, PREVZ, RADIUS, RETURN, SRPM, SEQCTR, SFM, SPIFLG, SPNTAB, TAPSTO, TOOLNO, TR ANSX, TRANSY, GTRANSZ, WORDS, BUFPRT, IDUM, ZCAM

DIMENSION MACH (12), PNOFLG(14), MTABLE(10), BUFFER (50), COMSTO(13), IFEDTAB (50), FORMAT ( 14), INTAP(20), OPTAB (50), PREBU (14), SPNTAB (100),

C 2TAPSTO (250), WORDS $(14)$, BUFPKT $(20), 1$ OUM(50)

CALL COMTI

WRITE DUTPUT TAPE $6,101,($ COMSTO $(\mathrm{J}), \mathrm{J}=1,13)$

LINCTR=LINCTR+1

RETURN

101 FORMAT (5X13A6)

END

* FORTRAN

* LaBel

CI3ON COMPUT

SUBROUT INE COMPUT

20130111

20130113

20130117

20130123

20130125

20130201

20130205

20130209

20130103

20130105

20130107

20130109

20130111

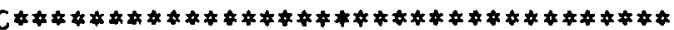

$\mathrm{C}$

C... SYSTEM COMMON, DIMENSION, AND EQUIVALENCS+STATEMENTS DIMENSION SYSTEM (1700)

C

EQUIVALENCE (SYSTEM(1700),KOM (1700),KFLAGS(1692), TAPETB(1622))

C

EQUIVALENCE (TAPETB(18),PROTAP(18), CANTAP (17),CLTAPE (16),

1 POCTAP (15), PLOTAP (14), SRFTAP (13), L IBTAP (12), CRDTAP $(11)$,

2 CORTAPI 9 ),

3 TAPES1 181 , TAPES $2($ 7), TAPES3 $(6)$, TAPES $4(5)$,

$C$

FORTIN( 41 , INTAPEI 31 , IOUTAP( 21 , PUNTAP( 11$)$

EQUIVALENCE (KFLAGST 70$)$,
KO (60),FO (60),K
$\begin{array}{ll}(50), F O & (160), K 1 \\ (56), K 5 & (55), K 6\end{array}$
( $591, \mathrm{~K} 2$
$(58), K 3$
$(57)$,
$K 4(56), K 5$
$(54), K 7$
( 53$), \mathrm{KB}$
1521 ,
KFLAGO ( 46), KFLAG1( 45), KFLAG2 ( 44), KFLAG3( 43), KFLAG4 ( 42$)$, IWAVEN ( 40$)$, IPTNLY( 39 ), NOPOST 38 ), INVERT( 37$)$, KAUTOP ( 36$)$,
ICLPRT( 35), INDEXX( 34), IPLOTRI 33), NOPLOT 311 . KDYNFG( 30 ), LOCJPT( 29), LOCBEG 28 ), KSECIN( 27$)$, NCLREC ( 26); LOCMAC( 25), LENSPC( 241 ,
9 IPOSTP( 201 , NUMPST 191$)$

C

C

EQUIVALENCE (KOM (8), KAPTID(1),KAPTIO(2), KAPTTR(3), KAPTCN(4))

COMMON SYSTEM

C

DIMENSION KFLAGS $(1), I P O S T P(1), K O M(1)$

C

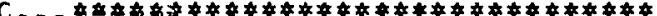

C COMMON AND OIMENSION-POSITIONING POST-PROCESSORS

COMMON MACH. NLOOP, NLOOPI, PNOFLG, MTABLE, NID, BITS, BUFFER, COMSTO, 
1CTRDLY,CTRFLG, FEDFL G, FEDIPR, FEDT AB, FORMAT, GGL, INT AP, IND2, IND3,

20130105

2KOMENT, KOOL, KX, KY, KZ, L INCTR, NAXIS, NCOOL, NFEEDC, NPRINT,

20130111

3NODAT, NSPIN, NTEST, NTOOL 1 ; NTOOL 2 ; NTRANS, NWPR, NZCAM, OPTAB,

20130113

4PREBU ,PRESTX, PRESTY PRESTZ, PREVX, PREVY, PREVZ, RADIUS, RETURN,

20130117

5RPM, SEQCTR, SFM, SP IFLG, SPNTAB, TAPSTO; TOOLNO, TRANSX, TRANSY,

20130123

GTRANSZ, WORDS, BUFPRT, IDUM, ZCAM

DIMENSION MACH (12), PNOFLG 14 , MTABLE (10), BUFFER (50), COMSTO (13),

20130125

C

$N T=M T A B L E(6)$

DELMAX $=0$

IF(IDUM $(15)) 12,13,12$

20130209

$12 \operatorname{IDUM}(15)=0$

GO TO 3

13 IF (PREVX-BITSI5, 1,5

1 IF (PREVY-BITSI 5,2,5

2 PREST $X=T A P S T O(K X)$

PRESTY $=$ TAPSTO $(K Y)$

PRESTZ=TAPSTO(KZ) + OPTAB(28) - OPTAB(29)

OPTAB $(2)=$ PRESTX

OPTAB (3) = PRESTY

OPTAB $(4)=$ PREST 2

3 RETURN

5 PRESTX $=$ TAPSTO $(K X)$

PRESTY $=$ TAPSTO $(K Y)$

PRESTZ=TAPSTO(KZ) + OPTAB(28) - OPTAB (29)

20130113

OPTAB $(2)=$ =PRESTX-PREVX

OPTAB $(3)=$ PRESTY-PREVY

OPTAB ( 4$)=$ PRESTZ-PREVZ

10 IF ( (ABSF (OPTAB ( 2$))$-OPTAB (16) $15,15: 11$

i1 DELMAX $=1$

IF (NT-1) $15,14,15$

20130102

PTAB $(2)=0 P$ TAB $(2) / 2.0$

PREST $X=P R E V X+O P T A B(2)$

15 IF ( (ABSF(OPTAB (3) ) )-OPTAB $(17)) 25,25,20$

20 DELMAX $=1$

IF(NT-1) $25,21,25$

21 OPTAB ( 3 ) =OPTAB (3)/2.0

PRESTY = PREVY +OPTAB (3)

25 IF IOPTAB (15)-2.0735,35,26

26 IF( (ABSF(OPTAB (4)))-OPTAB ( 18$)) 35,35,30$

30 DELMAX $=1$

IF (NT-1) $35,31,35$

20130103

20130105

20130107

20130109

31 OPTAB (4) =OPTAB $(4) / 2.0$

PREST $Z=P R E V Z+O P T A B(4)$

35 IF(DELMAX) 3,3,32

32 IF (NT-1) 37, 36,37

36 NTEST $=$ NTEST -3

$K X=K X-3$.

$K Y=K Y-3$

$K Z=K Z-3$

CTRFLG $=1.0$

20130115

20130117

37 KOMENT $=13$

GO TO 3 END

*1400

COMT 1

ENTRY COMTI 


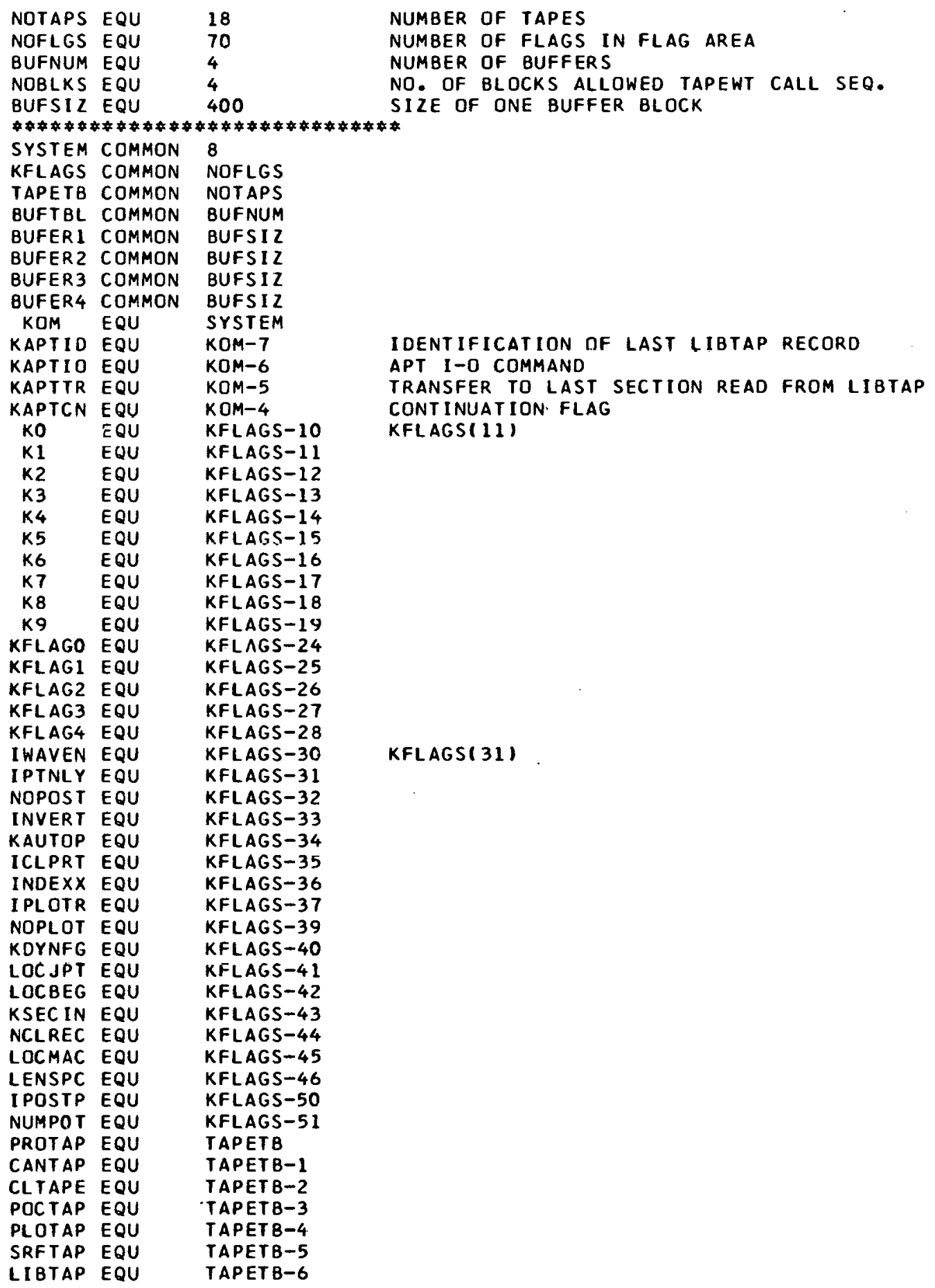




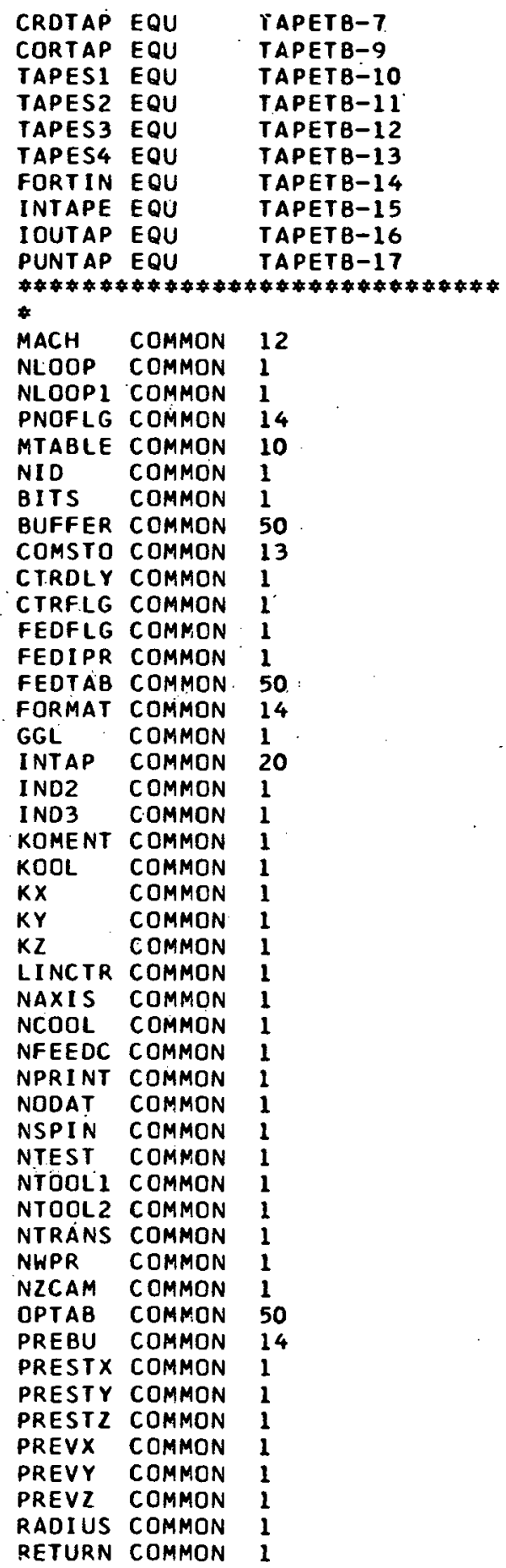




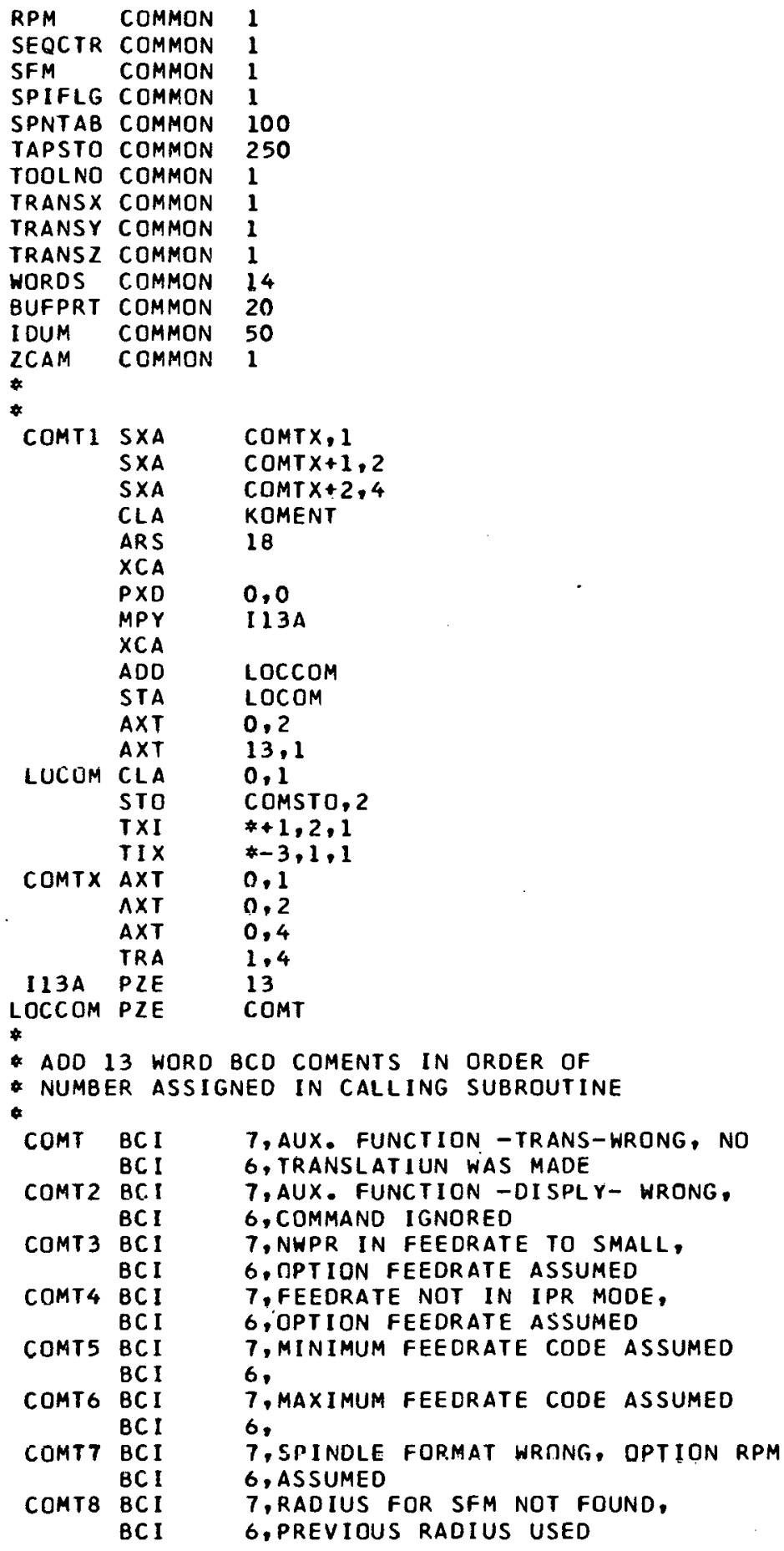




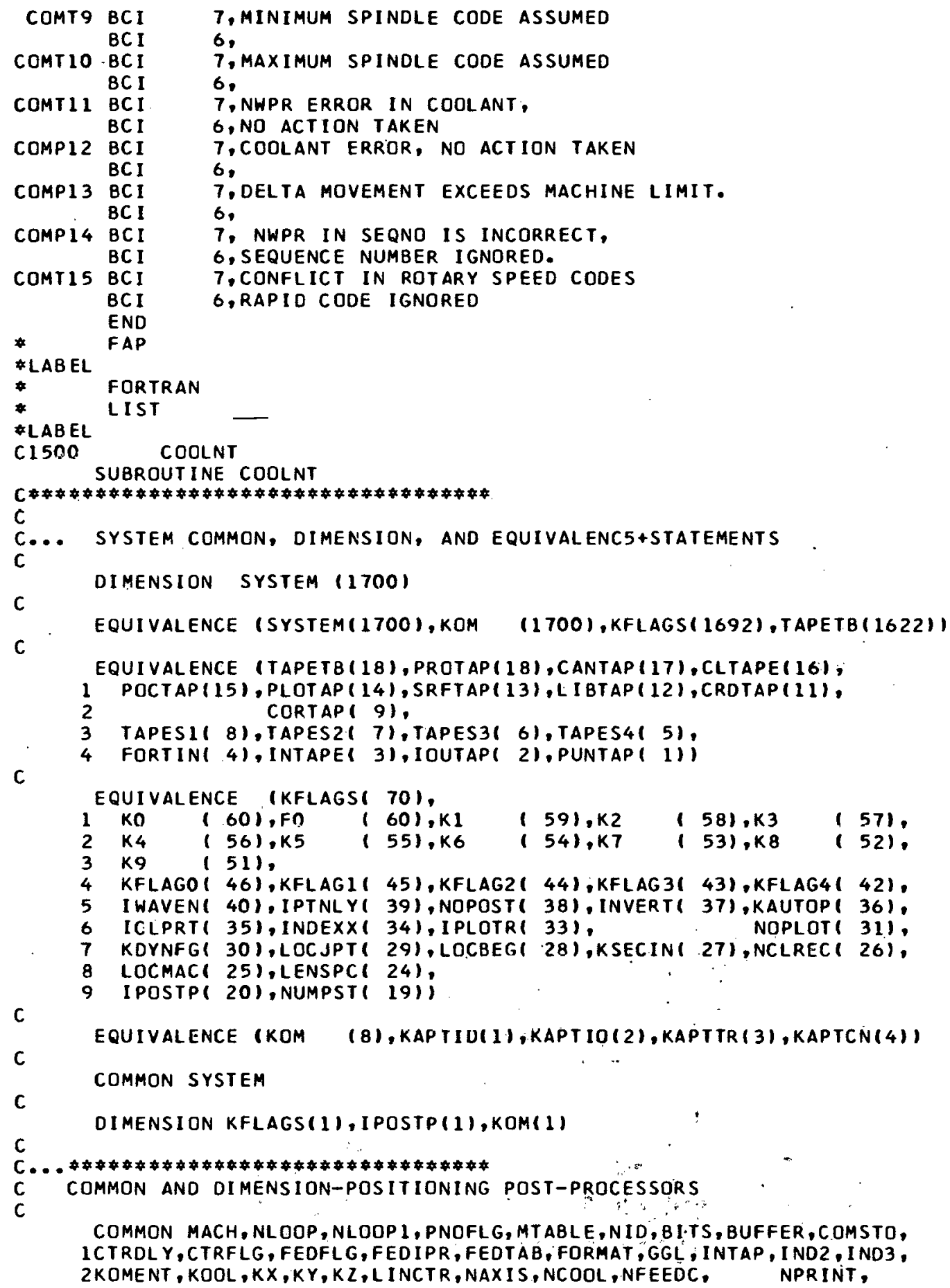

COMMON MACH, NLOOP, NLOOP I, PNOFLG, MTABLE, NID, BI.TS, BUFFER, COMSTO, ICTRDLY, CTRFLG, FEDFLG, FEDIPR, FEDTAB, FORMAT, GGL, INTAP, IND2, I ND3, 2KOMENT, KOOL, KX,KY,KZ,LINCTR, NAXIS, NCOOL, NFEEDC, NPRINT, 
3NODAT, NSPIN, NTEST, NTOOL 1, NTOOL 2, NTRANS, NWPR, NZCAM, OPTAB,

20130113

4PREBU, PRESTX, PRESTY, PRESTZ, PREVX, PREVY, PREVZ, RADIUS, RETURN,

5RPM, SEQCTR, SFM, SPIFLG, SPNTAB, TAPSTO, TOOLNO, TRANSX, TRANSY,

20130123

GTRANSZ, WORDS, BUFPRT, IDUM, ZCAM

20130125

DIMENSION MACH $(12)$, PNOFLG $(14)$, MTABLE $(10), B U F F E R(50)$, COMSTO (13), 20130201

I FEDTAB $(50)$, FORMAT $(14)$, INTAP $(20)$, OPTAB (50), PRE BU $(14)$, SPNTAB $(100)$, 2 TAPSTO (250), WORDS $(14)$, BUFPRT $(20), \operatorname{IDUM}(50)$

20130205

$N=M T A B L E(6)$

IF (NWPR-4) $1,5,5$

20130209

1 KOMENT $=11$

CALL COMENT

2 RETURN

5 IF (INTAP (4)-71)10,20,6

6 IF ( INTAP(4)-72) $2,30,7$

7 IF (INTAP (4)-89)10,20,8

8 IF (INTAP (4)-91) 21,20,10

21 I $F(N-13) 20,22,22$

$22 \mathrm{KOOL}=2$ GO TO 31

10 KOMENT $=12$

CALL COMENT

GO TO 2

$20 \mathrm{KOOL}=1$ GO TO 31

$30 \mathrm{KOOL}=0$

$31 \operatorname{IDUM}(30)=1$ OUM $(30)+1$ NCOOL $=1$ DUM $(30)$ IF(IDUM( 21$) 40,2,40$

40 GO TO $(2,2,2,42,2,2,2,41,2,42,2,2,42,2,2), N$

41 SEQCTR=SEQCTR+OPTAB (22)

B PREBU $(1)=606000000000$ GO TO 43

42 SEQCTR = SEQCTR+OPTAB (22) PREBU $(1)=$ SEQCTR

$43 \mathrm{NCOOL}=0$

PREBU $(14)=9-K O O L$

CALL CARD

CALL PRINT

GO TO 2

END

20130211

* fortran

* LIST

* LAB EL

4 FURTIN( 4 ), INTAPEI 3 ), IOUTAPI 2 ),PUNTAP ( 111 DATA

SUBROUTINE DATA

45721007

47490119

45721009

45721010

20130119

20130121

20130123

20130201

22130203

20130206

47490120

47490121

45721011

C1600

C

20130101

C... SYSTEM COMMON, DIMENSION, AND EQUIVALENC5+STATEMENTS DIMENSION SYSTEM (1700)

C

EQUIVALENCE (SYSTEM(1700),KOM (1700),KFLAGS(1692), TAPETB(1622))

EQUIYALENCE (TAPETB (18), PROTAP(18), CANTAP (17), CLTAPE (16),

POCTAP (15), PLOTAP (14), SRFTAP (13), LI BTAP $(12)$, CRUTAP (11),

CORTAPI 91 ,

TAPES1( 81 ,TAPES $2(7)$,TAPES 316$)$,TAPES41 51 , 
C

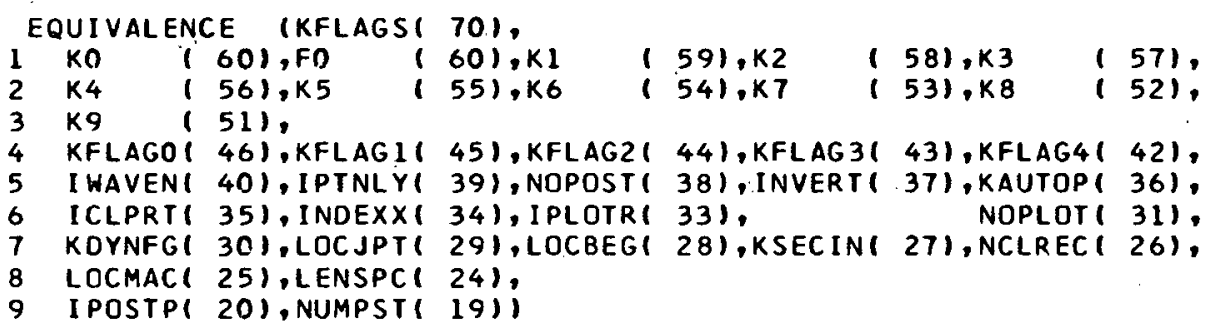

$\mathrm{C}$ EQUIVALENCE (KOM (8), KAPTIO(1), KAPTIO(2), KAPTTR(3), KAPTCN(4))

C COMMON SYSTEM

C DIMENSION KFLAGS(1), IPOSTP(1),KOM(1)

C

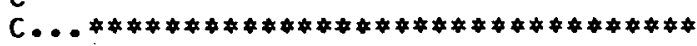

C COMMON AND DIMENSION-POSITIONING POST-PROCESSORS

C

COMMON MACH, NLOOP, NLOOPI, PNOFLG, MTABLE,NID, BITS, BUFFER, COMSTO, 1 CTRDL $Y, C T R F L G$, FEDFL G, FED IPR, FEDTAB, FORMAT, GGL, INTAP, IND2, IND3, 2KOMENT, KOOL, KX,KY,KZ, LINCTR, NAXIS, NCOOL, NFEEDC, NPRINT, 3 NODAT, NSPIN, NTEST, NTOOL 1 , NTOOL 2 , NTRANS, NWPR, NZCAM, OPTAB, 4PREBU ,PRESTX, PRESTY, PRESTZ, PREVX, PREVY, PREVZ, RADIUS, RETURN, SRPM, SEQCTR, SFM, SPIFLG, SPNTAB, TAPSTO, TOOLNO, TRANSX, TRANSY, 6TRANSZ, WORDS, BUFPRT, IDUM, ZCAM

DI MENSION MACH (12), PNOFLG (14), MTABLE(10), BUFFER(50),COMSTO(13), IFEDTAB ( 50$)$, FORMAT $(14)$, INTAP $(20)$, OPTAB (50), PREBU $(14)$, SPNTAB $(100)$,

C 2 TAPSTO $(250)$, WORDS $(14)$, BUFPRT $(20)$, I DUM(50)

20130101

20130105

20130111

20130113

20130117

20130123

20130125

20130201

20130205

20130209

30 CALL COMPUT

40 SEQCTR $=$ SEQC TR+OPT $A B(22)$ IF (CTRFLG) $1,2,1$

20130103

1 CTROLY $=1.0$ CTRFLG $=0.0$ GO TO 5

2 IF(CTRDLY) $3,5,3$

3 SEQCTR =SEQCTR-OPTAB (22) CTROL. $Y=0.0$ GO TO 5

5 CALL SETUP IF (PREBU (3)-BITS) 6,7,6

7 IF ( PREVX-BITS) $11,6,11$

$6 N=$ FORMAT (3)/10.0+SI GNF (.00 I, FORMAT (3) ) $A L=N$ $N=A B S F(F O R M A T(3)-(A 1 * 10.0))+.001$ $A 1=I N T F(P R E S T X *(10 . * * N)+S I G N F(.5, P R E S T X))$ PREVX $=A 1 /(10 . * * N)$

11 IF (PREBU (4)-BITS) $8,9,8$

9 IF (PREVY-BITS) $12,8,12$

8 N=FORMAT (4)/10.0+SIGNF $(.001$, FORMAT (4) ) $A 1=N$ $N=A B S F(F O R M A T(4)-(A 1 * 10.0))+.001$ $A I=I N T F(\operatorname{PRESTY} *(10 . * * N)+\operatorname{SIGNF}(\cdot 5, \operatorname{PRESTY}))$ PREVY $=A 1 /(10 . * N)$

12 IF (PREBU (5)-BITS) 10,20,10

20 IF(OPTAB $(15)-2.0) 15,15,14$ 


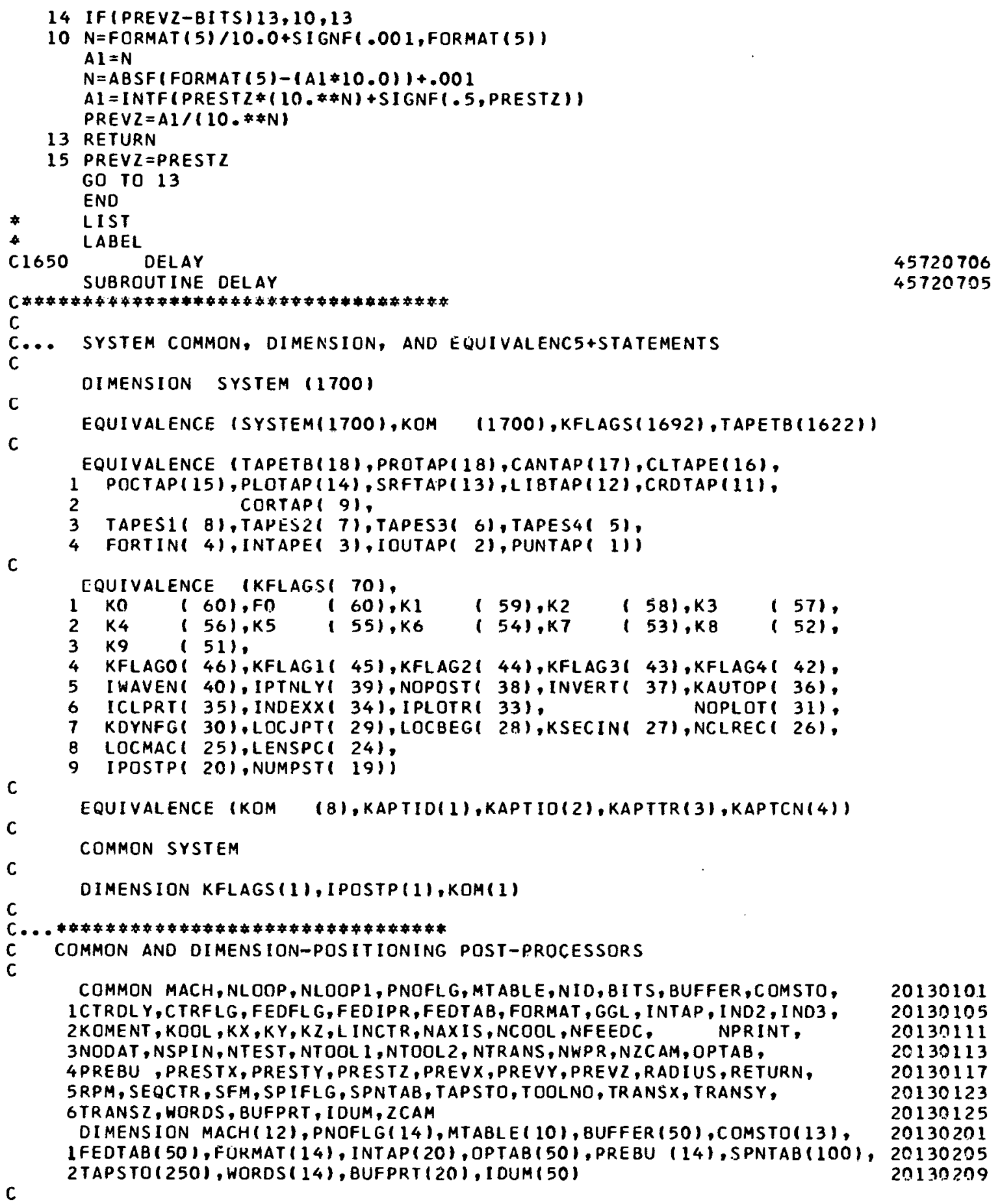




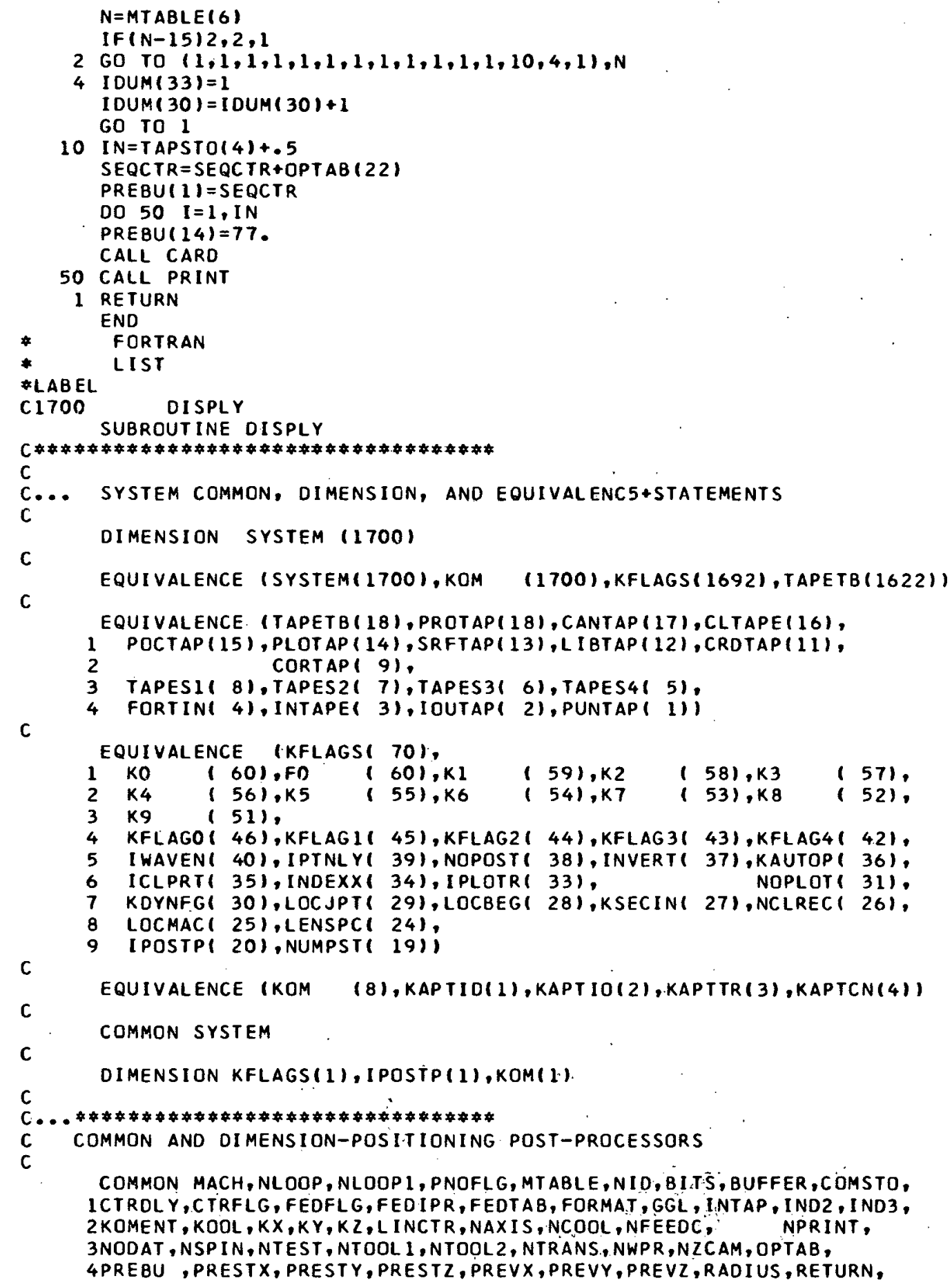

45720707 45720708 47490123 47490124 47490125 474901

45720710

45720712

45720713

45720711

45720714

45720715

45720716

45720717

45720718

20130101
20130101 20130105 20130111 20130113 
5RPM, SEOCTR, SFM, SPIFLG, SPNTAB, TAPSTO, TOOLNO, TRANSX, TRANSY, 6TRANSZ, WORDS, BUFPRT, I DUM, ZCAM

DIMENSION MACH (12), PNOFLG(14), MTABLE (10), BUFFER (50), COMSTO (13),

1 KOMENT $=2$

CALL COMENT

2 RETURN

3 ZCAM=TAPSTO(5)

$N Z C A M=1$

4 TOOLNO $=$ TAPSTO $(4)$

NTOOL $1=1$

NTOOL $2=1$

$N T=M T A B L E(6)$

IF (NT-15) $5,5,2$

5 Go TO $(2,2,2,2,2,2,2,2,6,7,2,2,2,2,2), \mathrm{NT}$

$6 \operatorname{OPTAB}(5)=0$.

GO TO 2

7 SEQCTR $=$ SEQCTR+OPTAB (22)

PREBU $(1)=S E Q C T R$

B PREBU(12) $=606360606060$

NTOOL $1=0$

NTOULL $2=0$

CALL CARD

CALL PRINT

GO TO 2

ENO

20130121

FORTRAN

20130123

* LIST

* LABEL

C1800 END

SUBROUTINE END

20130101

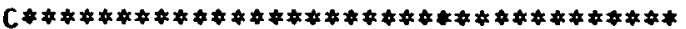

C

C... SYSTEM COMMON, DIMENSION, AND EQUIVALENC5+STATEMENTS

20130105

20130107

20130109

20130111

20130113

20130115

20130117

20130119

DIMENSION SYSTEM (1700)

EQUIVALENCE (SYSTEM(1700), KOM (1700), KFLAGS(1692), TAPETB(1622))

EQUIVALENCE (TAPETB $(18), \operatorname{PROTAP}(18), \operatorname{CANTAP}(17), \operatorname{CLTAPE}(16)$,

POCTAP (15), PLOTAP (14), SRFTAP (13), LIBTAP(12), CROTAP (11), CORTAP $(9)$,

TAPES $1($ 8), TAPES $2(7)$, TAPES31 6), TAPES4( 51,

C

FORTIN( 4$)$, INTAPE 31 , IOUTAPI 21 , PUNTAP ( 11

EQUIVALENCE (KFLAGS( 70$)$,
KO (60),FO ( 60$), K$
( 59$), \mathrm{K} 2$
$(58) . K 3$
(57),

K4 (56), K5

K9 ( 51$)$,

KFLAGO ( 46), KFLAG1 ( 45), KFLAG2 ( 44), KFLAG31 43), KFLAG4( 421 , IWAVEN( 40 ), IPTNLY( 39), NOPOST ( 38), INVERT( 37), KAUTOP( 36$)$, ICLPRT ( 35), INDEXX( 34), IPLOTR( 33), NOPLOT ( 31$)$, KDYNFG( 30$)$, LOCJPT( 29), LOCBEG( 28), KSECIN( 27), NCLREC( 26$)$, LOCMACI 25), LENSPC( 24),

C IPOSTP( 20), NUMPST( 19)) 


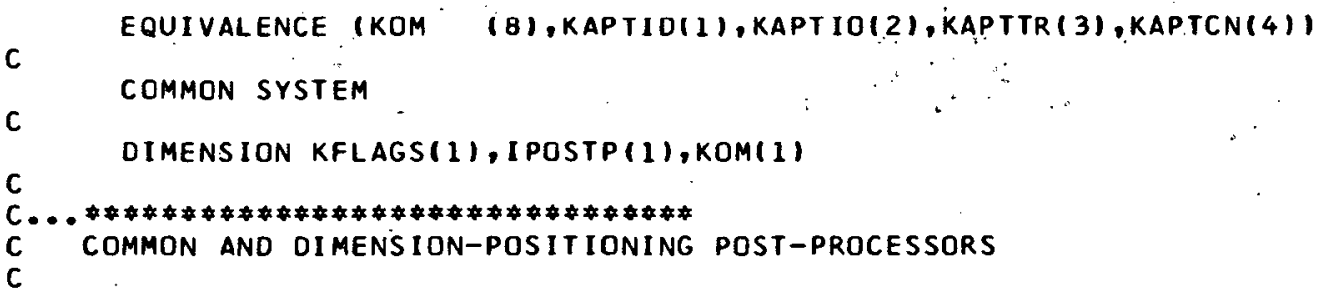

C COMMON MACH, NLOOP, NLOOPI, PNOFLG, MTABLE,NID, BITS, BUFFER, COMSTO, ICTROL $Y$, CTRFLG, FEDFL G, FED IPR, FEDTAB, FORMAT, GGL, INTAP, IND2, IND3, 2KOMENT, KOOL, KX, KY, KZ, LINCTR, NAXIS, NCOOL, NFEEDC, NPRINT, 3NODAT , NSP IN, NTEST, NTOOL 1 , NTOOL2, NTRANS, NWPR, NZCAM, OPTAB, 4PREBU ,PRESTX, PRESTY, PRESTZ, PREVX, PREVY, PREVZ, RADIUS, RETURN, 5RPM, SEQCTR, SFM, SPIFLG, SPNTAB, TAPSTO, TOOLNO, TRANSX, TRANSY, 6TRANSZ, WORDS, BUFPRT, IDUM, ZCAM

DIMENSION MACH (12), PNOFLG $(14)$, MTABLEI 10$),$ BUFFER $(50)$, COMSTO $(13)$, IFEDTAB (50), FORMAT ( 14), INTAP (20), OPTAB (50), PREBU (14), SPNTAB (100),

C 2TAPSTO $(250)$, WORDS $(14)$, BUFPRT(20), IDUM(50)

20130101 20130105 20130111 20130113 20130117 20130123 20130125 20130201 20130205 20130209

20130103

45720613 20130107 20130108

4 CALL ENDMA4 GO TO 20

6 CALL ENDMAG GO TO 20

10 PREBU $(14)=2$.

CALL CARO

CALL PRINT

$20 N N=-276$

$J=1$

$K=0$

CALL PUNCHA (NN, BUFFER, J,K)

1 RETURN

END

* fortran

* LIST

B EL

C1900 ENDMA4

SUBROUT INE ENDMA4

C

C

C... SYSTEM COMMON, DIMENSION, AND EQUIVALENC5+STATEMENTS

C

DIMENSION SYSTEM $(1700)$

C

EQUIVALENCE (SYSTEM(1700),KOM (1700),KFLAGS(1692),TAPETB(1622)

C

EQUIVALENCE (TAPETB(18), PROTAP( 18$),$ CANTAP (17), CLTAPE $(16)$,

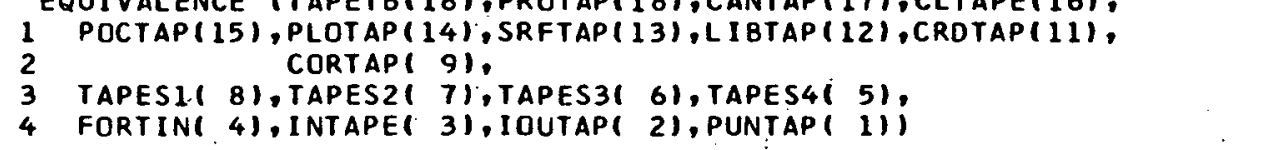

C

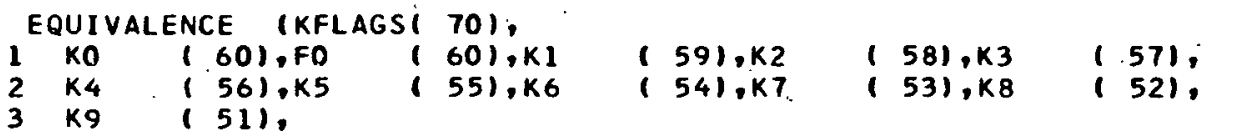

20130109 20139113

20130115

20130117

20130119

20130101
20130111 


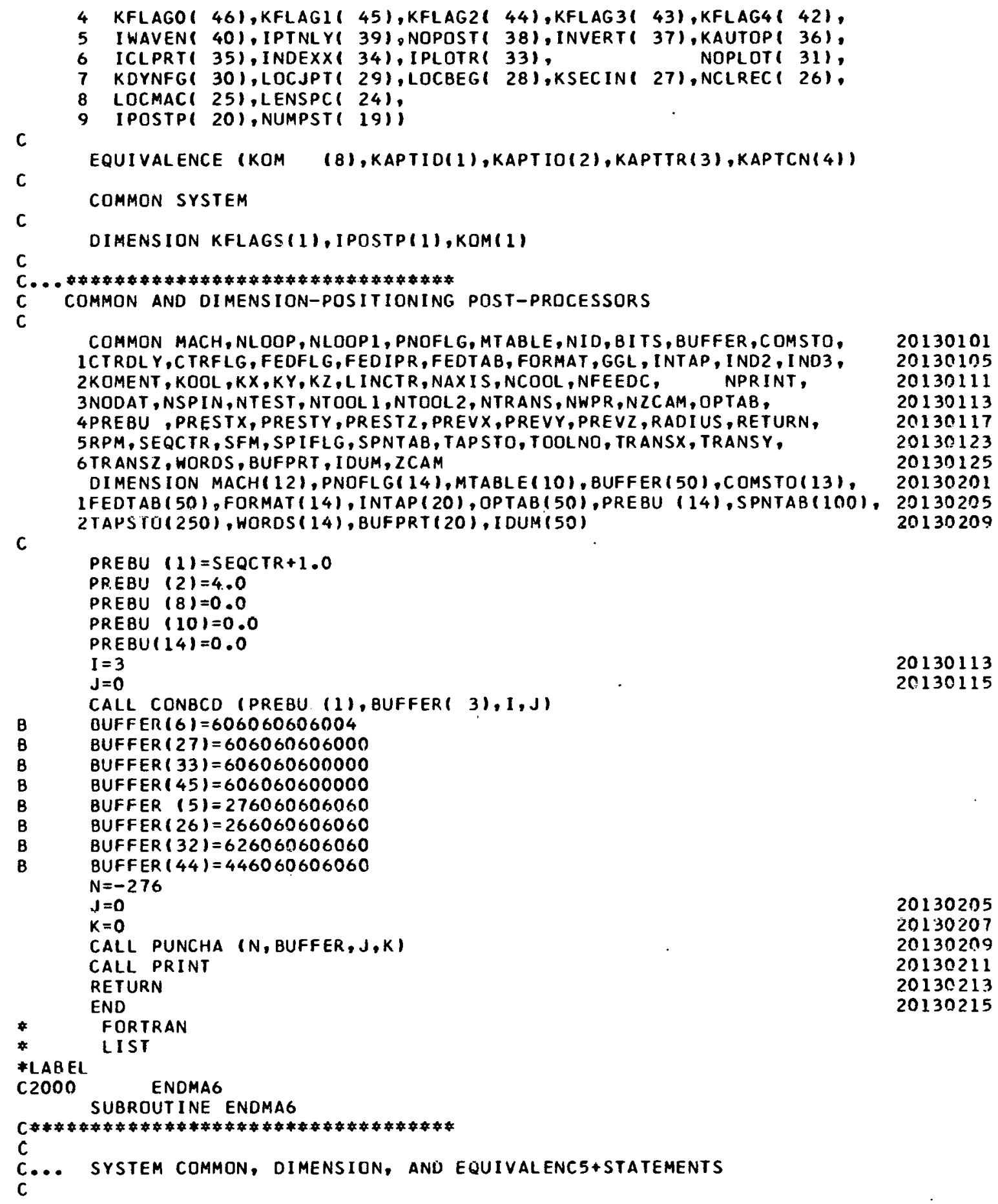




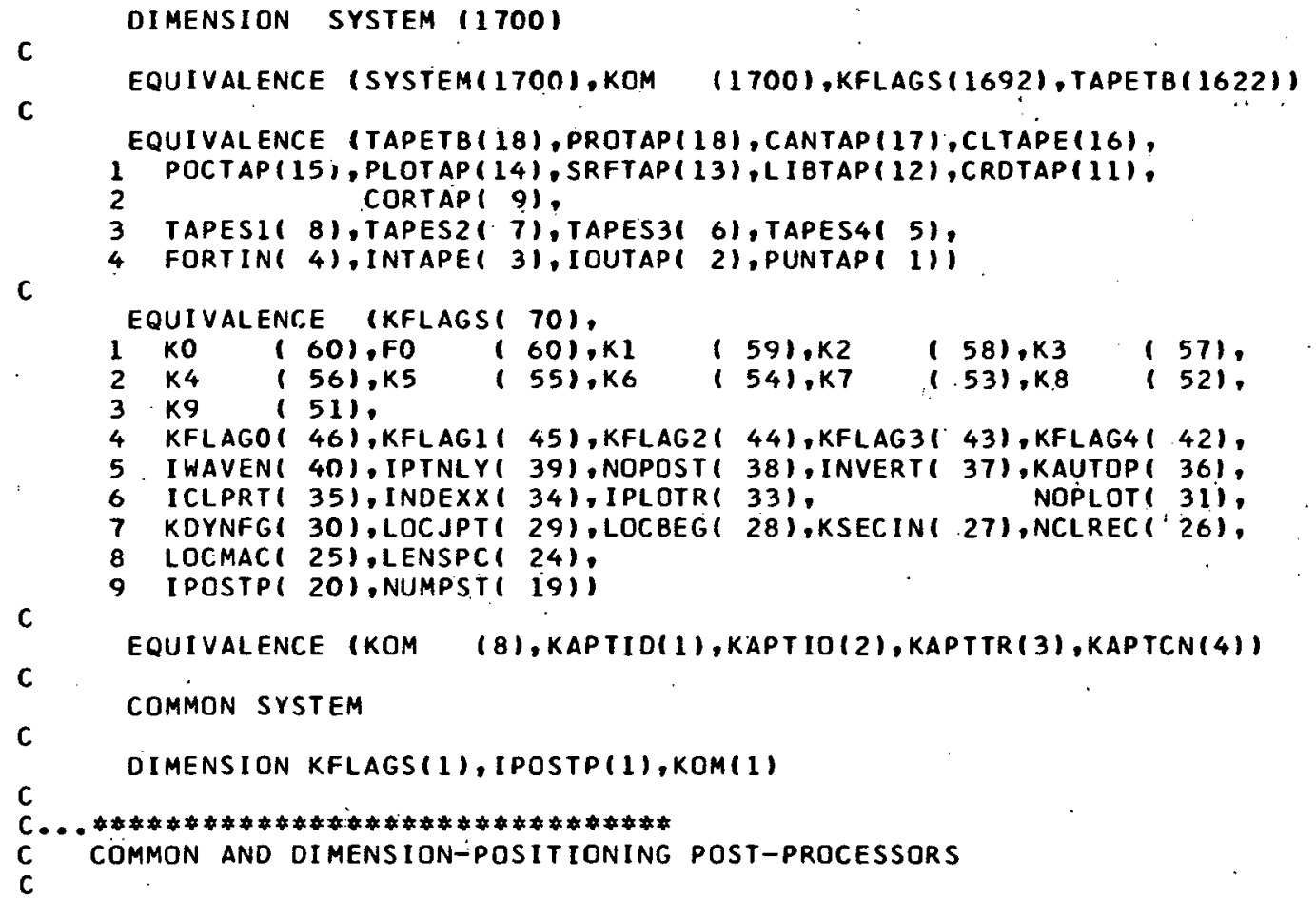

COMMON MACH,NLOOP, NLOOPI, PNOFLG, MTABLE,NID,BITS,BUFFER, COMSTO, 20130101 ICIRDL Y, CTRFLG, FEDFLG, FEDIPR, FEOTAB, FORMAT, GGL, INTAP, IND2; IND3, 20130105 2KOMENT, KOOL, KX, KY,KZ,LINCTR, NAXIS, NCOOL, NFEEDC, NPRINT, 3 NODAT, NSPIN, NTEST, NTOOL 1 , NTOOL 2 , NTRANS, NHPR, NZCAM, OPTAB, 4PREBU ,PRESTX, PRESTY, PREST Z, PREVX, PREVY, PREVZ, RADIUS, RETURN, SRPM. SEQCTR, SFM, SPI IFLG, SPNTAB, TAPSTO, TOOLNO, TRANSX, TRANSY, 6TRANSZ, WORDS, BUFPRT, I DUM, ZCAM DIMENS I ON MACH ( 12$)$, PNOFLG $(14)$, MTABLE $(10)$, BUFFER $(50)$, COMSTO $(13)$,

- IFEDTAB(50), FORMAT (14), INTAP (20), OPTAB (50), PREBU $(14)$, SPNTAB $(100)$. 2TAPSTO (250), WORDS $(14)$, BUFPRT $(20)$, I DUM(50) 20130111 20130113 20130117 20130123 20130125 20130201 20130205 20130209

C

DIMENSION HELP(4)

$D 02 \mathrm{~J}=1,3$

B $2 \operatorname{HELP(J)=000000000000}$

B $\quad$ HELP(4) $=536060606060$

$005 M=1,4$

$N=-24$

$J=0$

$K=0$

5 CALL PUNCHA $(N, H E L P, J, K)$

RETURN

END

- Fortran

* LIST

* $\mathrm{ABEEL}$

C2100 FEDC1

SUBROUTINE FEDC1

20130101 


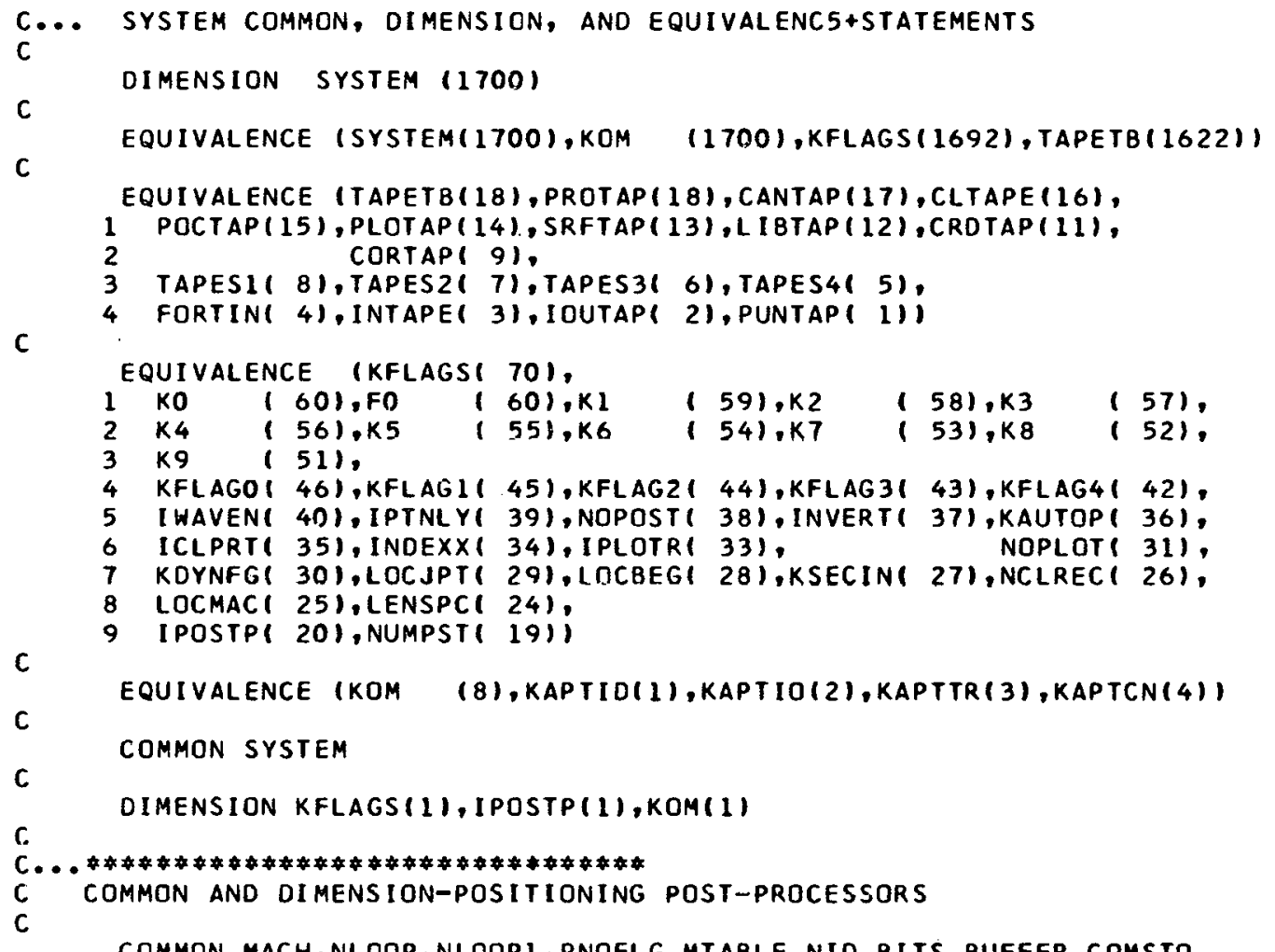
C

COMMON MACH, NLOOP, NLOOPI, PNOFLG, MTABLE, NID, BITS, BUFFER, COMSTO, ICTROLY, CTRFLG, FEOFLG, FEDIPR, FEOTAB, FORMAT, GGL, INTAP, IND2, IND3, 2KOMENT, KOOL, KX, KY, KZ,LINCTR, NAXIS, NCOOL, NFEEDC, NPRINT, 3NODAT, NSPI N, NTEST, NTOOL. 1 , NTOOL2, NTRANS, NWPR, NZCAM, OPTAB, 4PREBU, PRESTX, PRESTY, PRESTZ, PREVX, PREVY, PREVZ, RADIUS, RETURN, 5RPM, SEQCTR, SFM, SP IFLG, SPNTAB, TAPSTO, TOOLNO, TRANSX, TRANSY, 6TRANSZ, WORDS, BUFPRT, IDUM, ZCAM

DIMENSION MACH (12), PNOFLG( 14$)$, MTABLE $(10)$, BUFFER (50), COMSTO (13), IFEDTAB (50), FORMAT (14), INTAP $(20)$, OPTAB (50), PREBU $(14)$, SPNTAB $(100)$, 2TAPSTO $(250)$, WORDS $(14)$, BUFPRT.(20), I DUM(50)

20130101 20130105 20130111 20130113 20130117 20130123 20130125 20130201 20130205 20130209

IF ( FED I PR ) $30,31,30$

$30 I=O P T A B(6)$ I F(FED IPR-FEDTAB(I) ) $1,1,5$

1 KOMENT $=5$ NFEE DC $=$ I

2 CALL COMENT GO TO 21

3 RETURN

31 NFEEOC $=0$ GO TO 3

$5 I=O P T A B(14) * O P T A B(13)-O P T A B(13)+O P T A B(6)+.1$ I FIFED I PR-FEDTABI I) I0, 7,6

7 NFEEDC $=1$ GO TO 21

6 KOMENT $=6$ NFEEDC $=$ I
20130105

20130109

20130111

20130117 20130119 
GO TO 21

$10 K=O P T A B(6)$

$L=O P T A B(13)$

$0015 \mathrm{~J}=\mathrm{K}, \mathrm{I}, \mathrm{L}$

IF(FEDIPR-FEOTAB( J) )20,15,15

15 CONT INUE

16 ERROR=2.0

20130207

CALL PERROR (ERROR)

20 NFEEOC $=J-L$

21 CALL FEDCOD

GO TO 3

END

20130213

* fortran

* LIST

* LABEL

LIST

C2200 FEDCOD

SUBROUTINE FEDCOD

C

C

C... SYSTEM COMMON, DIMENSION, "AND EQUIVALENC5+STATEMENTS

C

C

DIMENSION SYSTEM $(1700)$

C

EQUIVALENCE (SYSTEM(1700), KOM (1700),KFLAGS(1692),TAPETB(1622))

EQUIVALENCE (TAPETB $(18)$, PROTAP(18), CANTAP(17), CLTAPE (16),

1 POCTAP (15), PLOTAP (14), SRFTAP (13), LI BTAP (12), CRDTAP (11).

2 CORTAP( 9$)$,

3 TAPES1( 8), TAPES $2(7)$, TAPES3 3 , 1 ,TAPES4(,5),

FORTIN( 4). INTAPE( 31 , IOUTAP( 2$)$, PUNTAP( 11$)$

EQUIVALENCE (KFLAGSI 70),

$\begin{array}{lllllll}1 & K O & (60), F O & (60), K 1 & (59), K 2 & (58), K 3 & (57), \\ 2 & K 4 & (56), K 5 & (55), K 6 & (54), K 7 & (53), K 8 & (52) !\end{array}$

$3 \mathrm{KO}(511)$

KFLAGO ( 46), KFLAGI ( 45), KFLAG2 ( 44), KFLAG31 43), KFLAG4 ( 42$)$, I WAVEN ( 40 ), IPTNLY( 39 ), NOPOST $(38)$, INVERT 37 ), KAUTOP( 36 ), ICLPRT ( 35), INDEXX( 34), IPLOTR ( 33), NOPLOT( 31$)$, KDYNFG( 30$),$ LOCJPT ( 29), LOCBEG ( 28), KSECINI 27), NCLREC ( 26),

LOCMAC ( 25), LENSPC( 24$)$,

9 IPOSTP( 20$)$, NUMPST( 19)!

C

(8), KAPTID(1), KAPTIO (2), KAPTTR(3), KAPTCN $(4))$

C

c COMMON SYSTEM

C

DIMENSION KFLAGS $(1), I P O S T P(1), \operatorname{KOM}(1)$

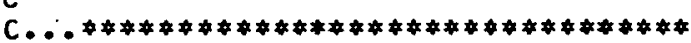

C COMMON ANO DIMENSION-POSITIONING POST-PROCESSORS

C

COMMON MACH, NLOOP, NLOOPI, PNOFLG, MTABLE, NID, BITS,BUFFER, COMSTO, ICTROLY, CTRFLG, FEDFLG, FEDIPR, FEDTAB, FORMAT, GGL, INTAP, IND2, IND3, 2KOMENT, KOOL, KX, KY, KZ,LINCTR, NAXIS, NCOOL, NFEEDC, NPRINT, 3NODAT, NSP I N, NTEST, NTOOL 1 , NTOOL 2 , NTRANS, NWPR, NZCAM, OPTAB; 4PREBU ,PRESTX, PRESTY, PRESTZ, PREVX, PREVY, PREVZ, RADIUS, RETURN, 5RPM, SEQCTR, SFM, SPI FLG, SPNTAB, TAPSTO, TOOLNO, TRANSX, TRANSY, 6IRANSZ; HORDS, BUFPRT, I DUM, ZCAM

DIMENSION MACH (12), PNOFLG (14), MTABLE( 10$)$, BUFFER (50),COMSTO (13), 
1FEDTAB (50), FORMAT (14), INTAP(20), OPTAB (50), PREBU (14), SPNTAB (100), 20130205

C 2 TAPSTO $(250)$, WORDS $(14)$, BUFPRT ( 20$)$, I DUM (50)

DIMENSION IFCODE(50)

$N T=M T A B L E(6)$

$N=N F E E D C$

IF (NT -15$) 2,2,1$

1 RETURN

2 GO TO $(1,1,1,1,1,1,1,8,9,10,1,1,1,14,1), N T$

8 NFEEDC $=\mathrm{N} / 2$

I $F(N F E E D C-7) 1,1,16$

$16 \mathrm{NFEEDC}=\mathrm{NFEEDC}+2$

GO TO 1

$9 \operatorname{IFCODE}(1)=110$

IFCODE (2) $=114$

IFCODE (3) $=116$

$\operatorname{IFCODE}(4)=120$

IFCOOE (5) $=128$

$\operatorname{IFCODE}(6)=136$

$\operatorname{IFCODE}(7)=141$

IFCODE (8) $=152$

IFCODE $(9)=181$

$\operatorname{IFCODE}(10)=210$

I $F C O D E(11)=212$

IFCODE $(12)=215$

I $F C O D E(13)=220$

I FCODE $(14)=226$

$\operatorname{IFCODE}(15)=230$

$\operatorname{IFCODE}(16)=238$

20 NFEEOC $=$ IFCODE (N)

GO TO 1

10 IF $(N) 11,1,11$

$11 \mathrm{NFEEDC}=(\mathrm{N}-1) / 3+1$ GO TO 1

$14 \operatorname{IFCODE}(1)=03$

$\operatorname{IFCODE}(2)=05$

IFCODE (3) $=06$

$\operatorname{IFCODE}(4)=08$

$\operatorname{IFCODE}(5)=10$

$\operatorname{IFCODE}(6)=12$

$\operatorname{IFCODE}(7)=13$

$\operatorname{IFCODE}(8)=15$

$\operatorname{IFCODE}(9)=17$

$\operatorname{IFCOOE}(10)=19$

$\operatorname{IFCOUE}(11)=20$

I $F C O D E(12)=22$

I FCODE $(13)=24$

$\operatorname{IFCOOE}(14)=25$

I FCODE $(15)=27$

$\operatorname{IFCOOE}(16)=29$

IFCODE $(17)=31$

I FCODE $(18)=32$

$\operatorname{IFCODE}(19)=34$

$\operatorname{IFCODE}(20)=36$

$\operatorname{IFCODE}(21)=37$

I FCOOE $(22)=39$

IFCODE $(23)=41$

I FCODE $(24)=43$

IFCODE $(25)=44$ 


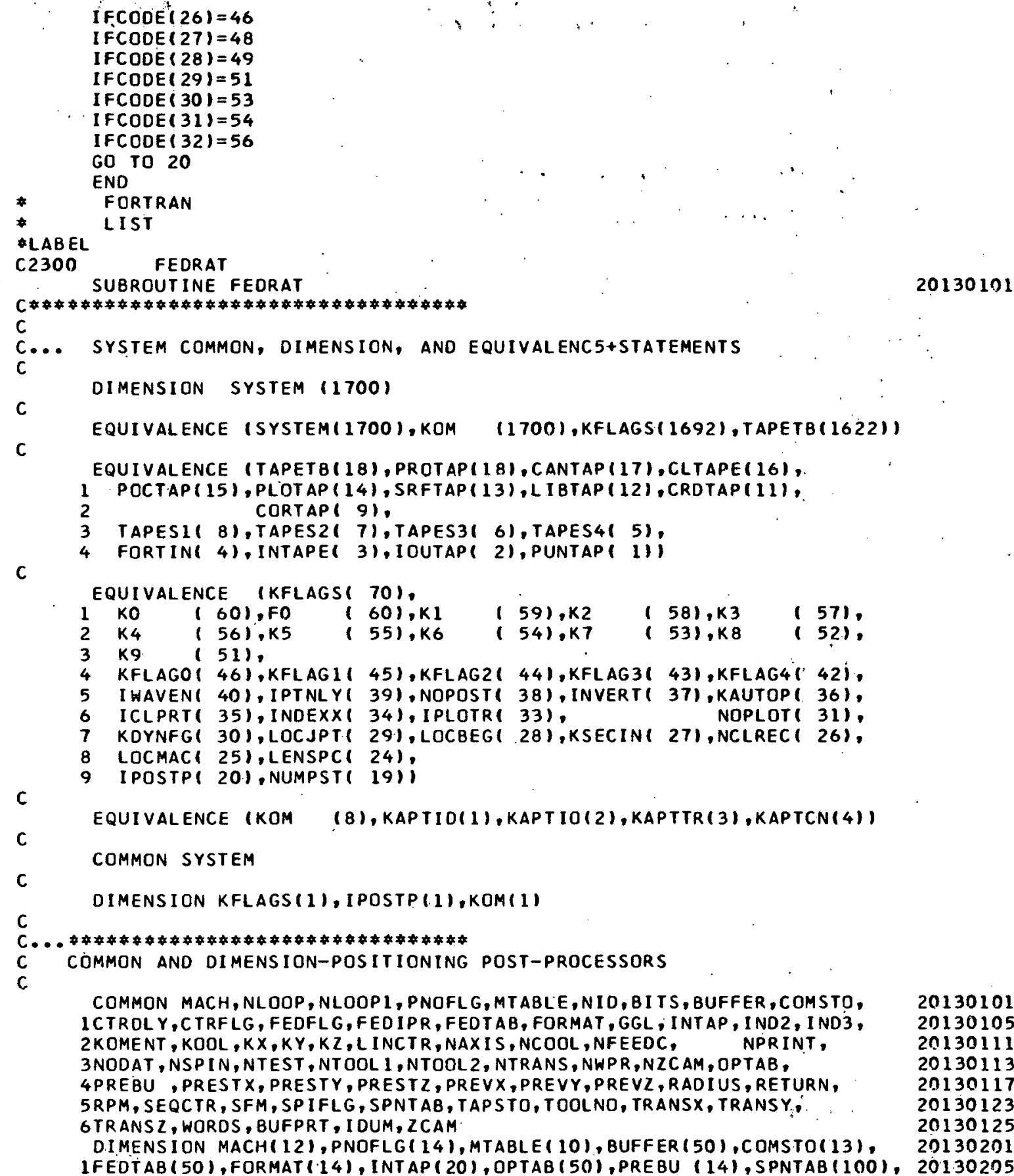

IFCODE $(26)=46$

I $F C O D E(27)=48$

FORTRAN

LIS

* LABEL

FEDRAT

0130101

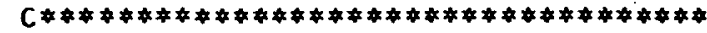

C... SYSTEM COMMON, DIMENSION, AND EQUIVALENC5+STATEMENTS

DIMENSION SYSTEM (1700)

EQUIVALENCE (TAPETB(18), PROTAP(18),CANTAP (17),CLTAPE 16$)$

$C$

C COMMON AND DIMENSION-POSITIONING POST-PROCESSORS

COMMON MACH, NLOOP, NLOOPL, PNOFLG, MTABLE, NID, BIIS, BUFFER, COMSTO, CTROLY,CTRFLG, FEDFLG, FED IPR, FEDTAB, FORMAT, GGL, INTAP, IND2, IND3, OTRANSZ, WORDS, BUFPRT, IDUM, ZCAM DEDTAB (50) ( 
1 KOMENT $=3$

21 CALL COMENT

2 FED I PR $=O P T A B(9)$

20130107

GO TO 10

70 IF(IDUM(28)) $7,7,14$

5 I F (INTAP ( 5$)-74) 6,7,13$

13 KOMENT $=4$ GO TO 21

6 IF(INTAP(5)-73)13,14,13

14 I $F(N-8) 40,15,40$

40 IF $(N-13) 13,15,15$

15 FED IPM $=$ TAPSTO $(4)$ FEDFLG $=1.0$ GÓ TO 9

27 I F $(N-13) 42,43,36$

43 I F(FED I PM-10.। $50,45,45$

50 IF IFED I PM-1.151,44,44

51 FEDI $P M=1$. KOMENT $=5$ CALL COMENT

44 NFEEDC $=400$ + FEDIPM $* 10$. GO TO 19

45 IFIFEDI PM-70.146,46,47

47 FEDI $P M=70$. KOMENT $=6$ CALL COMENT

46 NFEEDC $=500 .+$ FEDIPM 20130111 GO TO 19

42 NFEEDC =FEOIPM $\$ 2.0+.0001$ IF (NFEEDC-99)19,19,18

18 NFEEDC $=99$ KOMENT $=6$ CALL COMENT

19 FEDFLG $=1.0$ GO TO 30

7 FEDIPR $=$ TAPSTO(4)

IC FEDFLG $=1.0$ IF $(N-15) 11,11,20$

11 GO TO $(20,12,12,12,20,20,20,16,12,12,20,20,16,16,20), \mathrm{N}$

16 FEDIPM $=$ OPTAB $(26)$ \#FEDIPR I $F(N-13) 52,72,9$

45721109 47490201

52 IFIFEDI PM- $.5129,9,9$

72 IFIFEDIPM-1.173,9,9

73 FED IPM= 1 . GO TO 9

29 FEDIPM $=.5$

9 IF(NWPR 7$) 17,3,3$

3 IF(INTAP $(6)-145) 17,4,17$

4 IF(INTAP (7)-61)26,23,22

22 IF (INTAP ( 7)-62) $26,24,28$

28 IF(INTAP(7)-63) 26,25,26

23 TAPSTO $(7)=2$. GO TO 26

24 TAPSTR $(7)=3$. GO TO 26

25 TAPSTO $(7)=1$

26 IF (TAPSTO(7)-OPTAB (13)-.118,17,17

20130119 20130120

47490203

47490204

45721116 45721117

45721118

45721112 


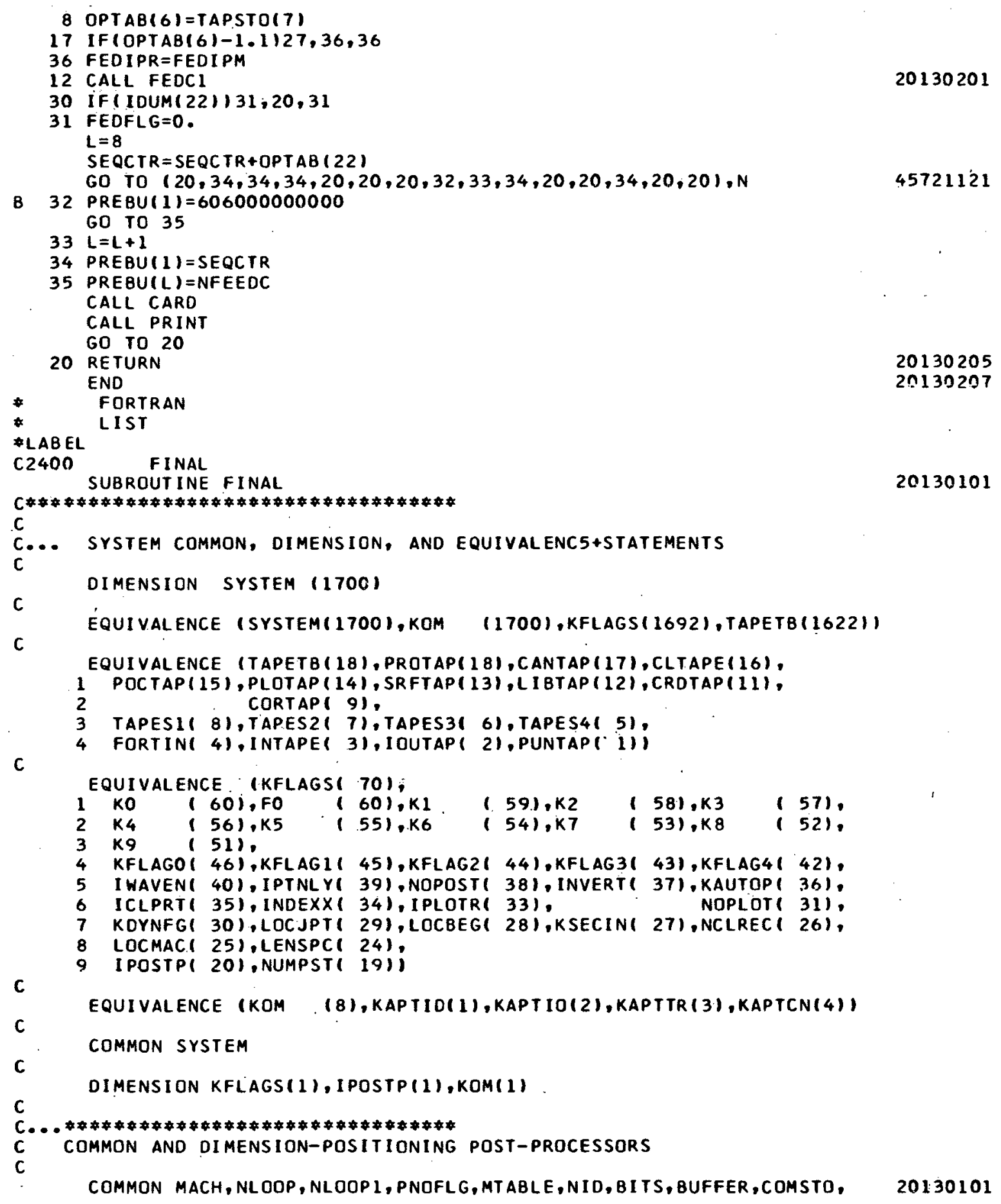




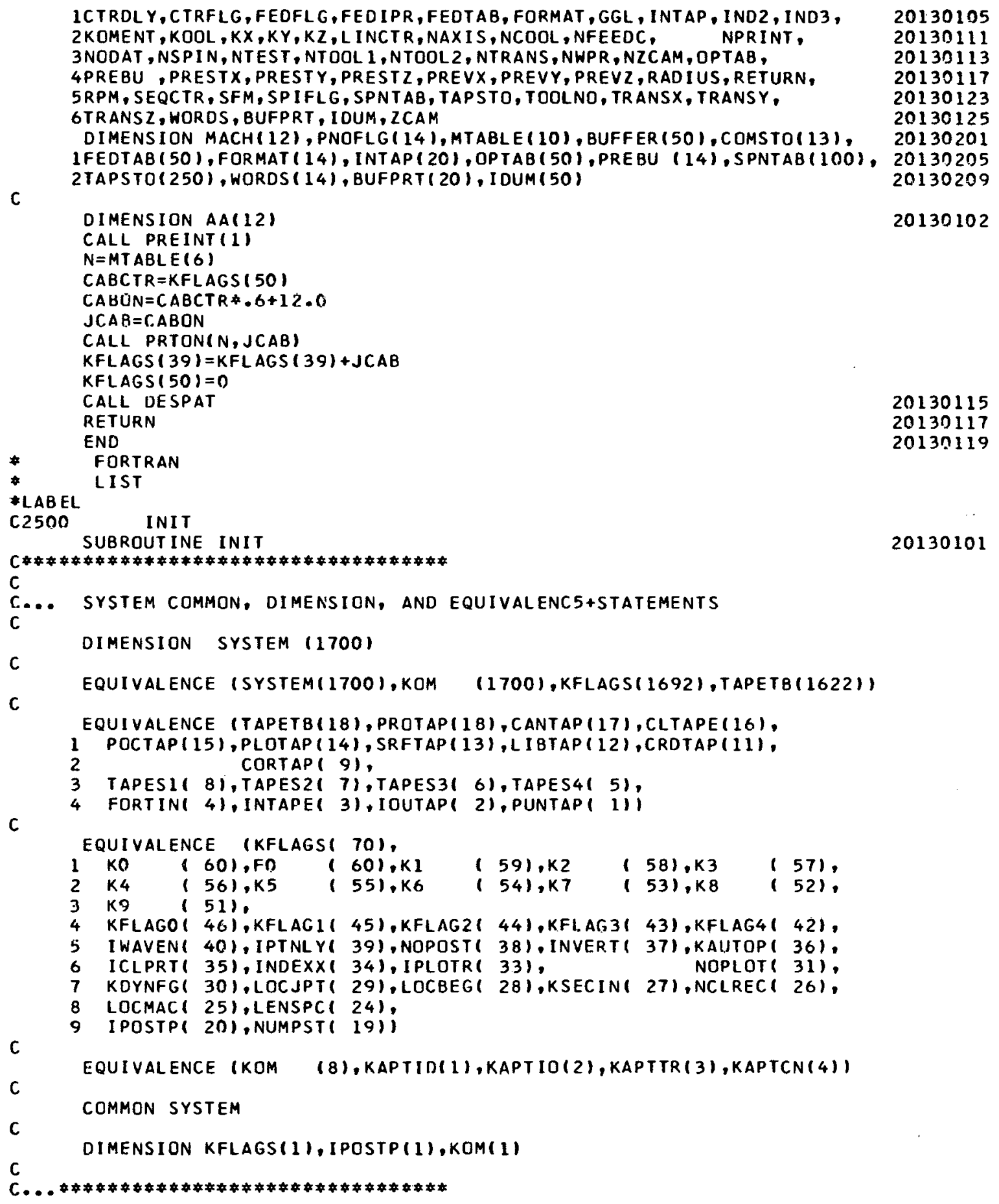


C

COMMON AND DIMENSION-POSITIONING POST-PROCESSORS

C

COMMON MACH, NLOOP, NLOOP1, PNOFLG, MTABLE,NID,BITS, BUFFER, COMSTO, 20130101 1CTRDLY, CTRFLG, FEDFLG, FEDIPR, FEDTAB, FORMAT, GGL, INTAP, IND2, IND3, 20130105 $2 K O M E N T, K O O L, K X, K Y, K Z$, LINCTR, NAXIS, NCOOL, NFEEDC, NPRINT, 3 NODAT, NSPIN, NTEST, NTOOL 1, NTOOL2, NTRANS, NHPR, NZCAM, OPTAB, 20130111 4PREBU , PRESTX, PRESTY, PRESTZ, PREVX, PREVY, PREVZ, RADIUS, RETURN, 20130113 20130117 5RPM, SEQCTR, SFM, SPIFLG, SPNTAB, TAPSTO, TOOLNO, TRANSX, TRANSY, 6TRANSZ, WORDS, BUFPRT, I DUM, ZCAM

DIMENSION MACH $(12)$, PNOFLG $(14)$, MTABLE $(10)$, BUFFER $(50)$, COMSTO $(13)$, 20130123 20130125 2TAPSTO (250), WORDS $(14)$, BUFPRT $(20)$, IDUM(50)

20130209

CALL INITI

20130103

DO $30 \quad I=1,14$

B WOROS(I) $=606060606060$

FORMAT $(I)=B I T S$

30 PREBU $(I)=B I T S$

$O P T A B(6)=1.0$

$O P T A B(7)=1.0$

$O P T A B(22)=1.0$

OPTAB $(23)=B$ ITS

IDUM ( 29) =1

$N=M \cdot T A B L E(6)$

If (N-15)18,18,19

20130105

18 GO TO $(1,2,3,4,5,6,7,8,9,10,11,12,13,14,15), N$

1 CALL MACHO1

GO TO 20

2 CALL MACHO2

GO TO 20

3 CALL MACHO3

GO TO 20

4 CALL MACHO 4

GO TO 20

5 CALL MACHOS

GO TO 20

6 CALL MACHOG

GO TO 20

7 CALL MACHOG GO TO 20

8 CALL MACHOB GO TO 20

9 CALL MACHO9

GO TO 20

10 CALL MACHIO

GO TO 20

11 CALL MACHII

GO TO 20

12 CALL MACHI2

GO TO 20

13 CALL MACH13 GO TO 20

45720118

45720119

$4 \mathrm{CALL} \mathrm{MACH} 14$

47490206

GO TO 20

15 GO TO 20

19 ERROR $=5.0$

CALL PERROR (ERROR)

20 RE TURN

20130109

20130111

20130113

20130115

20130117

20130119

20130121

20130123

END

20130124

20130125 


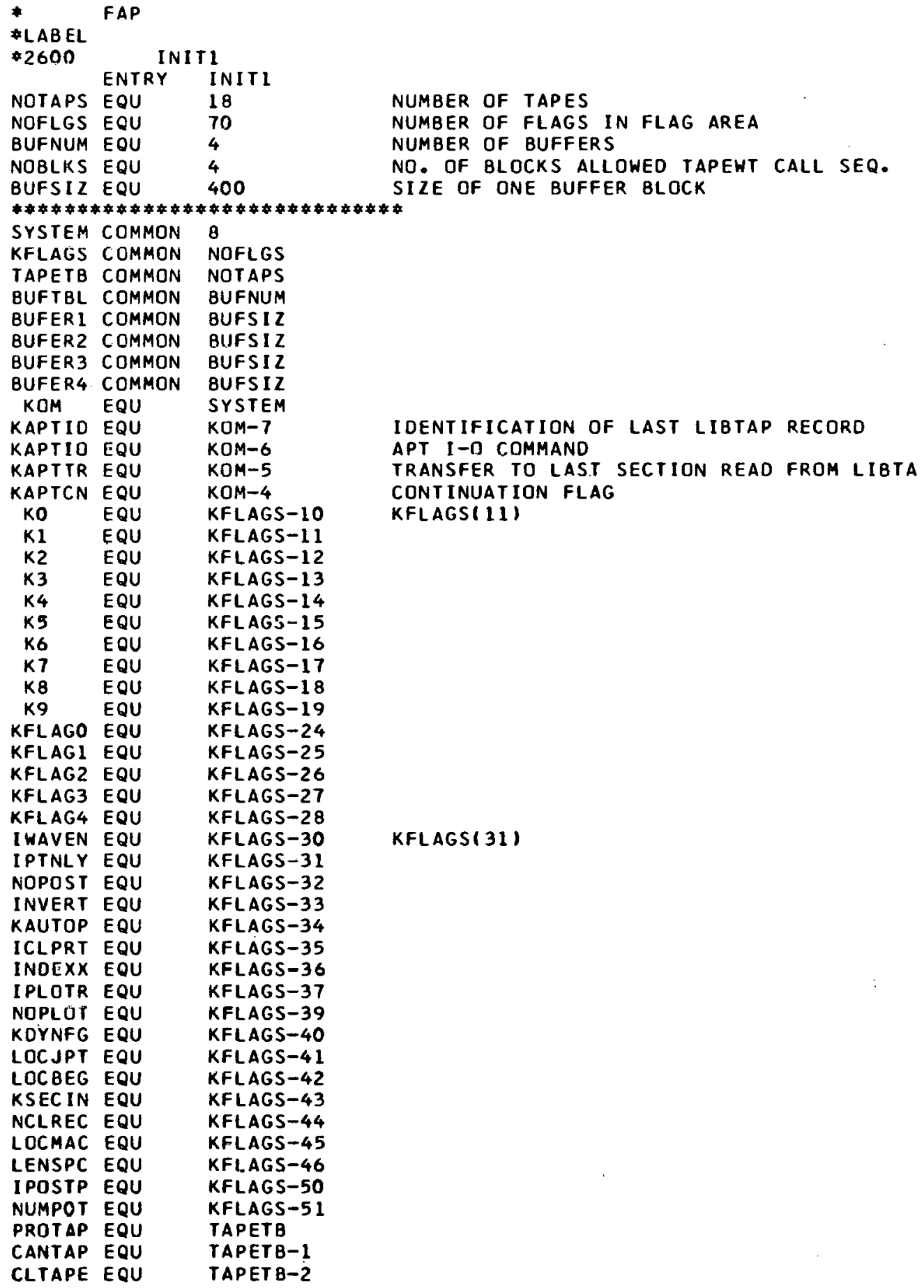




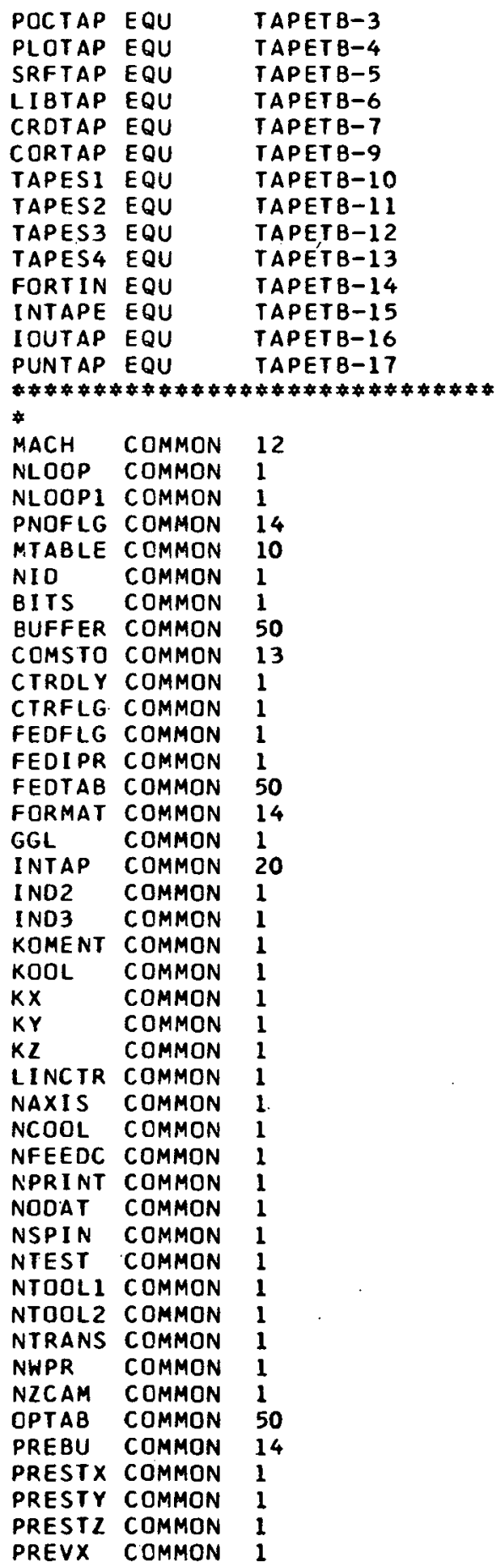




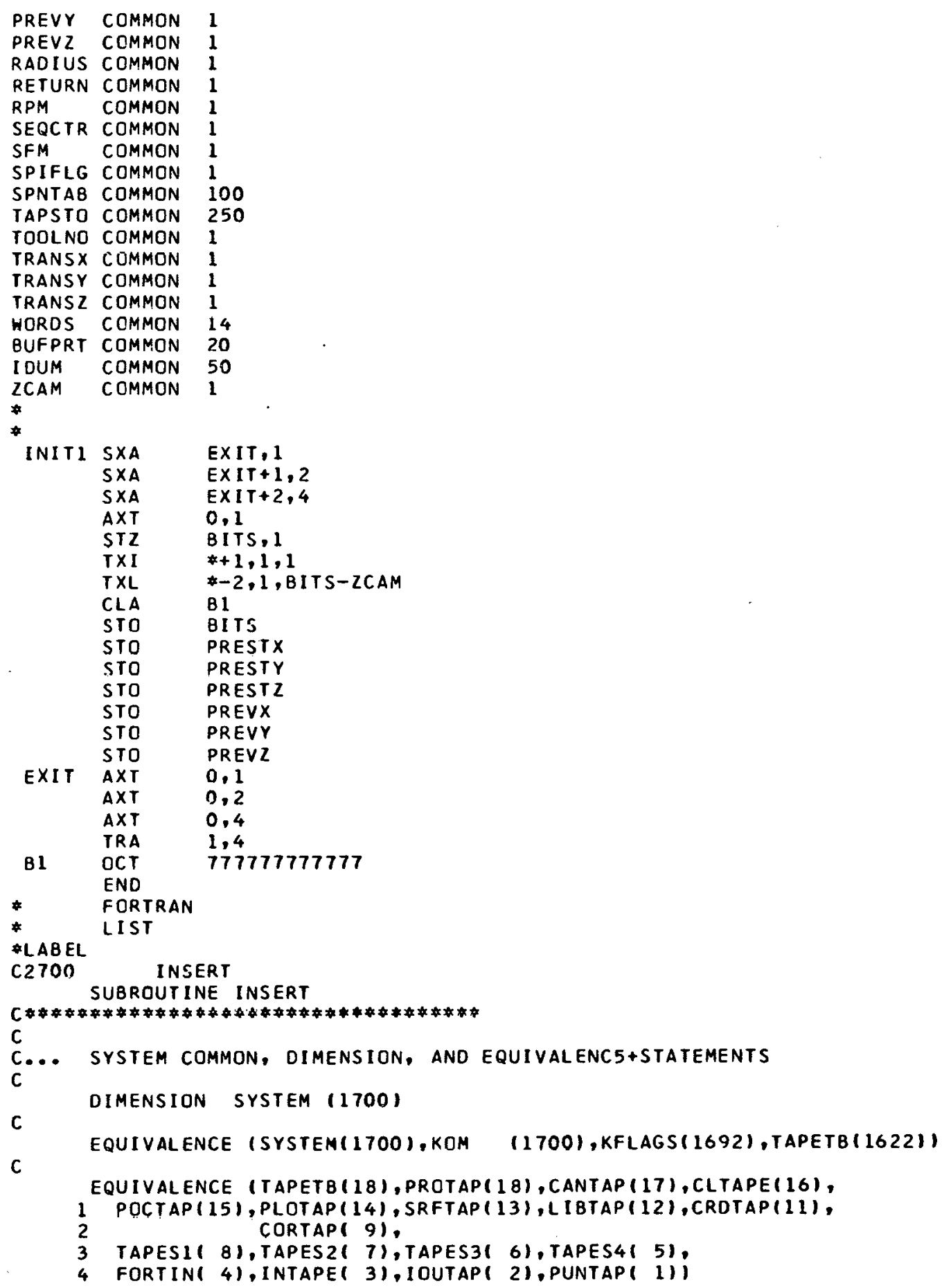


C

$\begin{array}{lllllll}\text { EQUIVALENCE } & \text { (KFLAGS }(70), \\ 1 & \text { KO } & (60), \text { FO } & (60), K 1 & (59), K 2 & (58), K 3 & (57), \\ 2 & K 4 & (56), K 5 & (55), K 6 & (54), K 7 & (53), K 8 & (52),\end{array}$

$3 K 9(51)$,

4 KFLAGO ( 46), KFLAG1 ( 45), KFLAG21 44), KFLAG3( 431 , KFLAG4 ( 421 , I HAVEN( 40 ), IPTNLY( 39), NOPOST ( 38), INVERT ( 37), KAUTOP( 36$)$, ICLPRT ( 35), INDEXX( 34), IPLOTR( 33), NOPLOT 31 ), 7 KOYNFG( 30$)$, LOCJPT( 29$)$, LOCBEG 28 ), KSECIN( 271 , NCLREC 261 , 8 LOCMAC ( 25), LENSPC ( 24),

C IPOSTP( 20), NUMPST( 19)

C

EQUIVALENCE (KOM (8), KAPTID(1), KAPTIO(2), KAPTTR(3), KAPTCN(4))

C COMMON SYSTEM

C DIMENSION KFLAGS 11 , I POSTP(1),KOM(1)

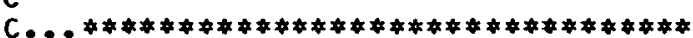

C COMMON AND DIMENSION-POSITIONING POST-PROCESSORS

$\mathrm{C}$

COMMON MACH, NLOOP, NLOOPI, PNOFLG, MTABLE, NID, BITS, BUFFER, COMSTO, 1 CTROL Y, CTRFLG, FEDFL G, FED IPR, FEDTAB, FORMAT, GGL, INTAP, IND2, IND3, 2 KOMENT, KOOL, KX, KY, KZ, LINCTR, NAXIS, NCOOL, NFEEDC, NPRINT, 3NODAT, NSPIN, NTEST, NTOOL 1 , NTOOL 2 , NTRANS, NWPR, NZCAM, OPTAB, 4PREBU, PRESTX, PRESTY, PRESTZ, PREVX, PREVY, PREVZ, RADIUS, RETURN, 5RPM, SEQCTR, SFM, SP IFLG, SPNTAB, TAPSTO, TOOLNO, TRANSX, TRANSY, 6TRANSZ, WORDS, BUFPRT, I DUM, ZCAM

DIMENSION MACH (12), PNOFLG $(14)$, MTABLE $(10), B U F F E R(50), C O M S T O(13)$, IFEDTAB (50), FORMAT (14), INTAP (20), OPTAB (50), PREBU $(14)$, SPNTAB(100),

C 2 TAPSTO $(250)$, WORDS $(14)$, BUFPRT $(20), 1$ DUM $(50)$

20130101 20130105 20130111 20130113 20130117 20130123 20130125 20130201 20130205 20130209

CALL PUNCHA1-66,TAPSTO $(4), 0,0)$

CALL MESSAG

RETURN

END

$+$

FORTRAN

* LIST

C2730 LEADER

SUBROUT INE LEADER

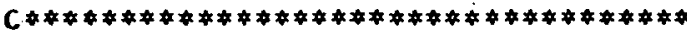

$C$

C... SYSTEM COMMON, DIMENSION, AND EQUIVALENC5+STATEMENTS

C

DIMENSION SYSTEM (1700)

C

EQUIVALENCE (SYSTEM(1700), KOM (1700),KFLAGS(1692), TAPETB(1622)

C

EQUIVALENCE (TAPETB (18), PROTAP (18),CANTAP (17), CLTAPE $(16)$, 1 POCTAP (15), PLOTAP (14), SRFTAP (13), LIBTAP $(12)$, CROTAP $(11)$,

CORTAP ( 9$)$,

3 TAPES 11 81, TAPES $2($ 7), TAPES3( 6$)$,TAPES 4151,

C

EQUIVALENCE (KFLAGS( 70$)$,
$1 \mathrm{KO}$
( 60), FO
( 60$), K 1$
( 56$), \mathrm{K} 5$
$(55), K 6$. ( 54$), K 7$
( 51 ).
KFLAGO ( 46), KFLAGI (45), KFLAG2 144
$591, k 2 \cdot 1581,1 k 3$
( $531, K 8$
(57).
$2 K 4$

4 


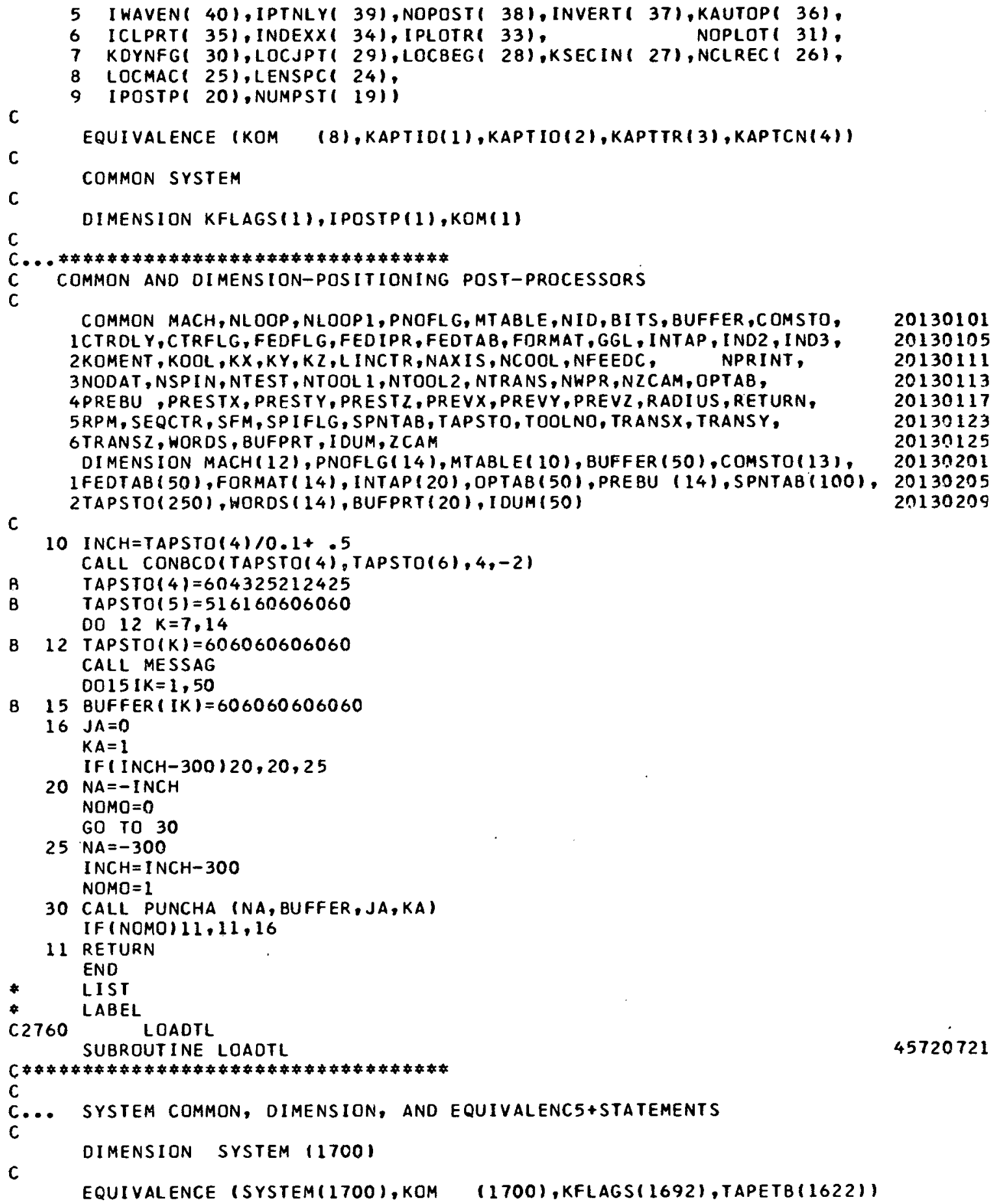


C

EQU IVALEÑCE (TAPPETB (18), PROTAP $(18), C A N T A P(17), C L T A P E(16)$, 1 POCTAP (15), PLOTAP (14), SRFTAP (13), LI BTAP $(12)$, CRDTAP $(11)$, CORTAP ( 9$)$

3 TAPES $1(8)$, TAPES $2(7)$, TAPES 3161 , TAPES $4(51$,

C FORTIN( 4$)$, INTAPE( 3 ), IOUTAP( 2$)$, PUNTAP( 11$)$

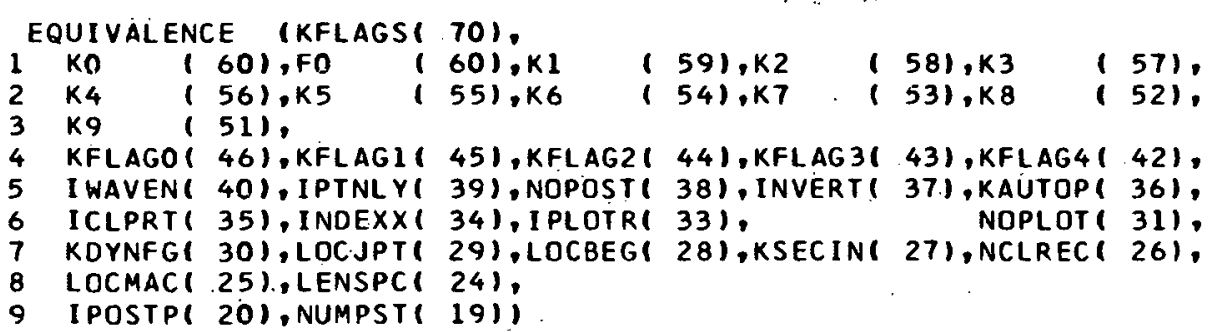

C

C

EQUIVALENCE (KOM (8), KAPTIO(1), KAPTIO(2),KAPTTR(3),KAPTCN(4))

C COMMON SYSTEM

C DIMENSION KFLAGS 11$),$ IPOSTP $(1), K O M(1)$

C . .

C COMMON AND DIMENSION-POSITIONING POST-PROCESSORS

C

C

COMMON MACH, NLOOP, NLOOP1, PNOFLG, MTABLE, NID, BITS, BUFFER, COMSTO, ICTRDLY, CTRFLG, FEDFLG, FED IPR , FEDTAB, FORMAT , GGL, INTAP, IND2, IND3, 2KOMENT, KOOL, KX,KY,KZ,L INCTR, NAXIS, NCOOL, NFEEDC, NPRINT, 3NODAT, NSPIN, NTEST, NTOOL 1 , NTOOL 2 , NTRANS, NWPR, NZCAM, OPTAB, 4PREBU , PRESTX, PRESTY, PRESTZ, PREVX, PREVY, PREVZ, RADIUS, RETURN, 5RPM, SEQCTR, SFM, SPI FLG, SPNTAB, TAPSTO, TOOLNO, TRANSX, TRANSY, 6TRANSZ, WORDS, BUFPRT, IDUM, ZCAM

DIMENSION MACH (12), PNOFLG (14), MTABLE (10), BUFFER (50),COMSTO 131 , IFEDTAB (50), FORMAT $(14)$, INTAP $(20)$, OPTAB $(50)$, PREBU $(14)$, SPNTAB $(100)$, 2TAPSTO(250), WORDS $(14)$, BUFPRT $(20)$, I DUM(50)

20130101 20130105 20130111 20130113 20130117 20130123 20130125 20130201 20130205 $2013 n 209$

45720722 45720723 IF $(N-15) 2,2,1$

2 GO TO $(1,1,1,1,1,1,1,1,1,1,1,1,10,30,1), N$

10 SEQCTR $=\$ E Q C T R+O P T A B(22)$ IF ( PREVZ -13.9985$) 21,20,20$

21 PREVZ $=13.999$

20 PREBU $(1)=$ SEQCTR PREBU (5) = PREVZ PREBU $(14)=6$. PREBU $(8)=599$. FEDFLG $=1$.

30 IF (NWPR-4) $1,31,32$

$31 \mathrm{NT}=4$ GO TO 34

$32 \mathrm{NT}=8$ OPTAB(28) = TAPSTO(10)

34 IF $(N-13) 9,33,9$

9 SEQCTR=SEQCTR+OPTAB (22) PREBU(1)=SEQCTR PREBU $(14)=6$. PREBU $(12)=T A P S T O(N)$
45720725

45720802 


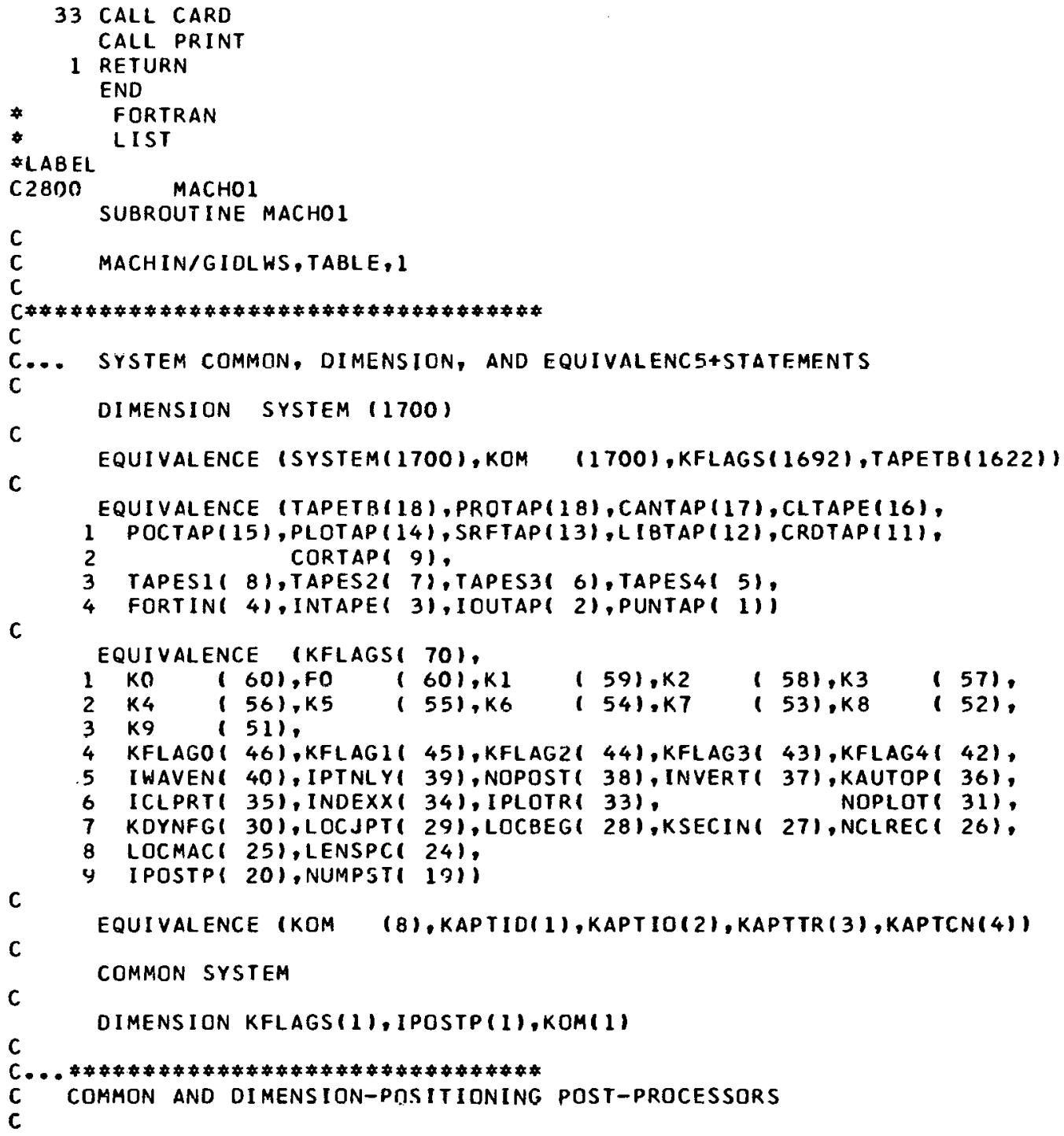

COMMON MACH, NLOOP, NLOOPI, PNOFLG, MTABLE, NID, BITS, BUFFER, COMSTO, ICTRDLY, CTRFLG, FEDFL G, FEDIPR, FEDTAB, FORMAT, GGL, INTAP, IND2, IND3, 2KOMENT, KOOL, KX,KY,KZ, LINCTR, NAXIS, NCOOL, NFEEDC, NPRINT, 3NODAT, NSPIN, NTEST, NTOOL 1 , NTOOL 2 , NTRANS, NWPR, NZCAM, DPTAB, 4PREBU ,PRESTX, PRESTY, PRESTZ, PREVX, PREVY, PREVZ, RADIUS, RETURN, 5RPM, SEQCTR, SFM, SP IFLG, SPNTAB, TAPSTO, TOOLNO, TRANSX, TRANSY, GTRANSZ, WOROS, BUFPRT, IDUM, ZCAM DIMENSIUN MACH(12), PNOFLG (14), MTABLE (10), BUFFER (50), COMSTO(13), IFEDTAB (50), FORMAT (14), INTAP (20), OPTAB (50), PREBU (14), SPNTAB (100),

C 2TAPSTO $(250)$, WORDS $(14)$, BUFPRT $(20)$, I DUM (50)

20130101 20130105 20130111 20130113 20130117 20130123 20130125 20130201 20130205 20130209 20130103 20130105 
2KOMENT, KOOL, KX, KY, KZ, L INCTR, NAXIS, NCOOL, NFEEDC,

NPRINT,

20130111

3NODAT, NSP IN, NTEST, NT OOL 1, NTOOL 2, NTRANS, NWPR , NZCAM, OPTAB,

4PREBU, PRESTX, PRESTY, PRESTZ, PREVX, PREVY, PREVZ, RADIUS, RETURN,

20130113

5RPM, SEQCTR, SFM, SPIFLG, SPNTAB, TAPSTO, TOOLND, TRANSX, TRANSY,

20130117

GIRANSZ, WORDS, BUFPRT, IDUM, ZCAM

20130123

20130125

DIMENS ION MACH (12), PNOFLG( 14 ), MTABLE( 10), BUFFER (50), COMSTO(13),

20130201

IFEDTAB (50), FORMAT (14), INTAP (20), OPTAB (50), PREBU (14), SPNTAB (100), 20130205

C

2 TAPSTO(250), WORDS $(14)$, BUFPRT $(20)$, I DUM (50)

20130209

FORMAT $(1)=30.0$

20130103

FORMAT $(3)=-64.0$

FORMAT $(4)=-64.0$

20130105

FORMAT $(5)=-64.0$

20130107

FORMAT $(8)=10.0$

20130109

FORMAT $(10)=20.0$

WURUS(I) $=606060604560$

WORDS $(3)=606760606060$

WORDS $(4)=607060606060$

WOROS $(5)=607160606060$

WORDS(8) $=606060606026$

$\operatorname{WORDS}(10)=606060606062$

WORDS $(14)=604460606060$

OPTAB $(9)=.002$

20130123

OPTAB $(10)=21.0$

20130125

$O P T \wedge B(11)=1.0$

OPTAB $(12)=16,0$

20130201

20130203

OPT $A B(13)=1.0$

$\operatorname{OPTAB}(14)=8.0$

$O P T A B(15)=2.0$

NAXIS =OPTAB (15)

20130205

20130207

20130209

20130211

$\operatorname{OPT} A B(16)=70.0$

20130213

$O P T A B(17)=56.0$

20130215

$\operatorname{IDUM}(14)=1.0$

SPNTAB $(1)=21.0$

20130219

20130221

SPNTAB $(2)=27.0$

20130223

20130225

20130301

20130303

20130305

20130307

20130309

20130311

20130313

20130315

20132317

20130319

20130321

20130323

20130401

20130403

20130405

20130407

20130409

20130411

$2013 n 413$

20130415

20130417

FEDTAB $(8)=.024$

RETURN

20130419 


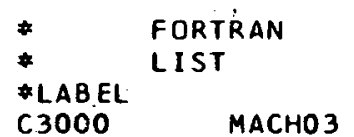

COMMON MACH, NLOOP, NLOOP I, PNOFLG, MTABLE, NID, BITS, BUFFER, COMSTO, ICTRDLY, CTRFLG, FEDFL G, FED IPR, FEDTAB, FORMAT, GGL, INTAP, IND2, IND3, 2KOMENT, KOOL, KX,KY,KZ, LINCTR, NAXIS, NCOOL, NFEEOC, NPRINT, 3NDDAT, NSPIN, NTEST, NTOOL 1 , NTOOL2, NTRANS, NWPR, NZCAM, OPTAB, 4PREBU ,PRESTX, PRESTY, PREST Z; PREVX, PREVY, PREVZ, RAO IUS, RETURN, SRPM, SEQCTR, SFM, SP IFLG, SPNTAB, TAPSTO, TOOLNO, TRANSX, TRANSY, GTRANS Z, WORDS, BUFPRT, I DUM, ZCAM

DIMENSION MACH (12), PNOFLG $(14)$, MTABLE $(10)$, BUFFER (50), COMSTO $(13)$, LFEDTAB (50), FORMAT (14), INTAP (20), OPTAB (50), PREBU (14), S PNTAB (10D), 2TAPSTO $(250)$, HORDS $(14)$, BUFPRT $(20)$, IDUM $(50)$

C

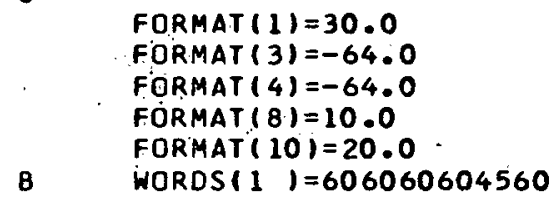




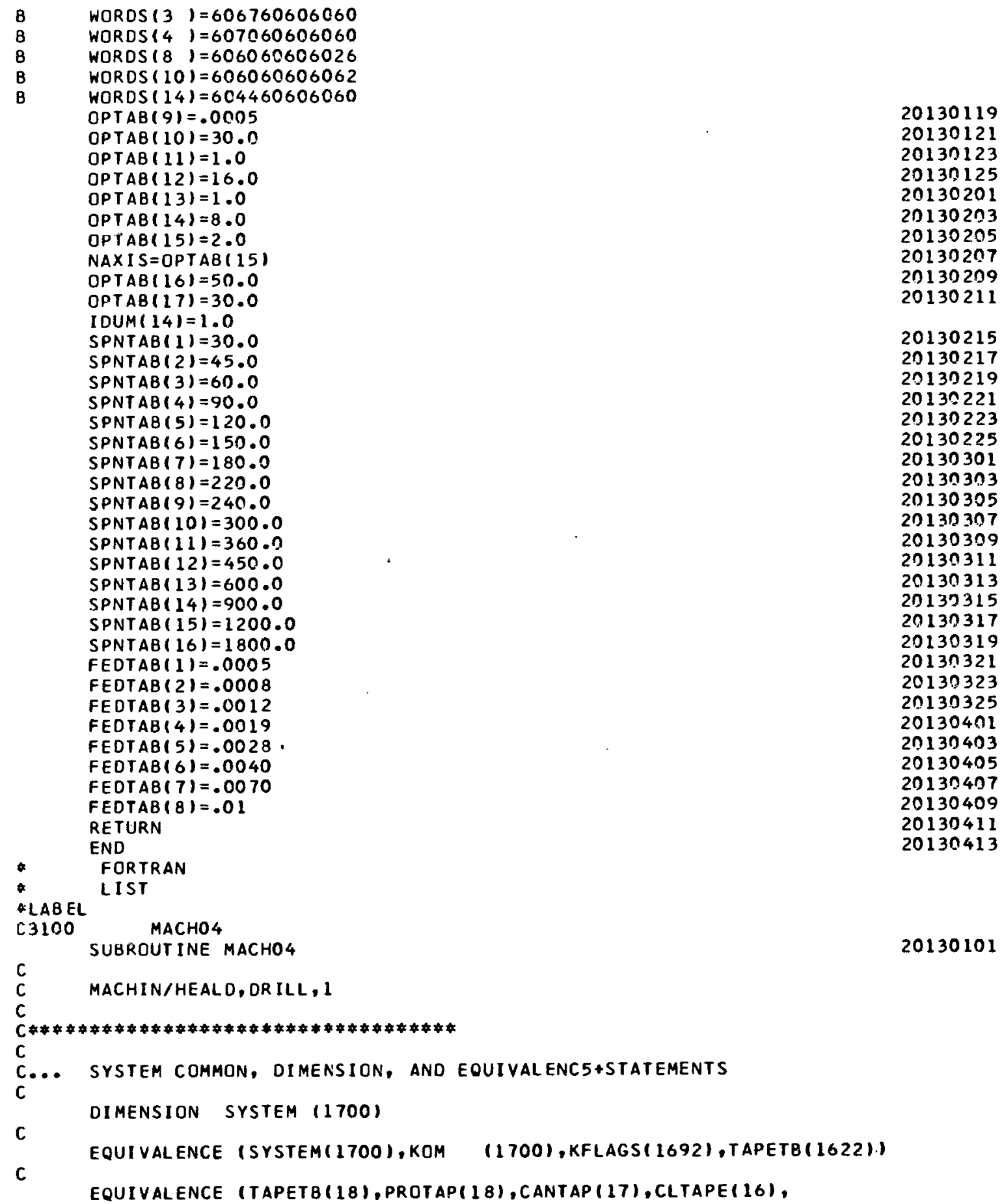




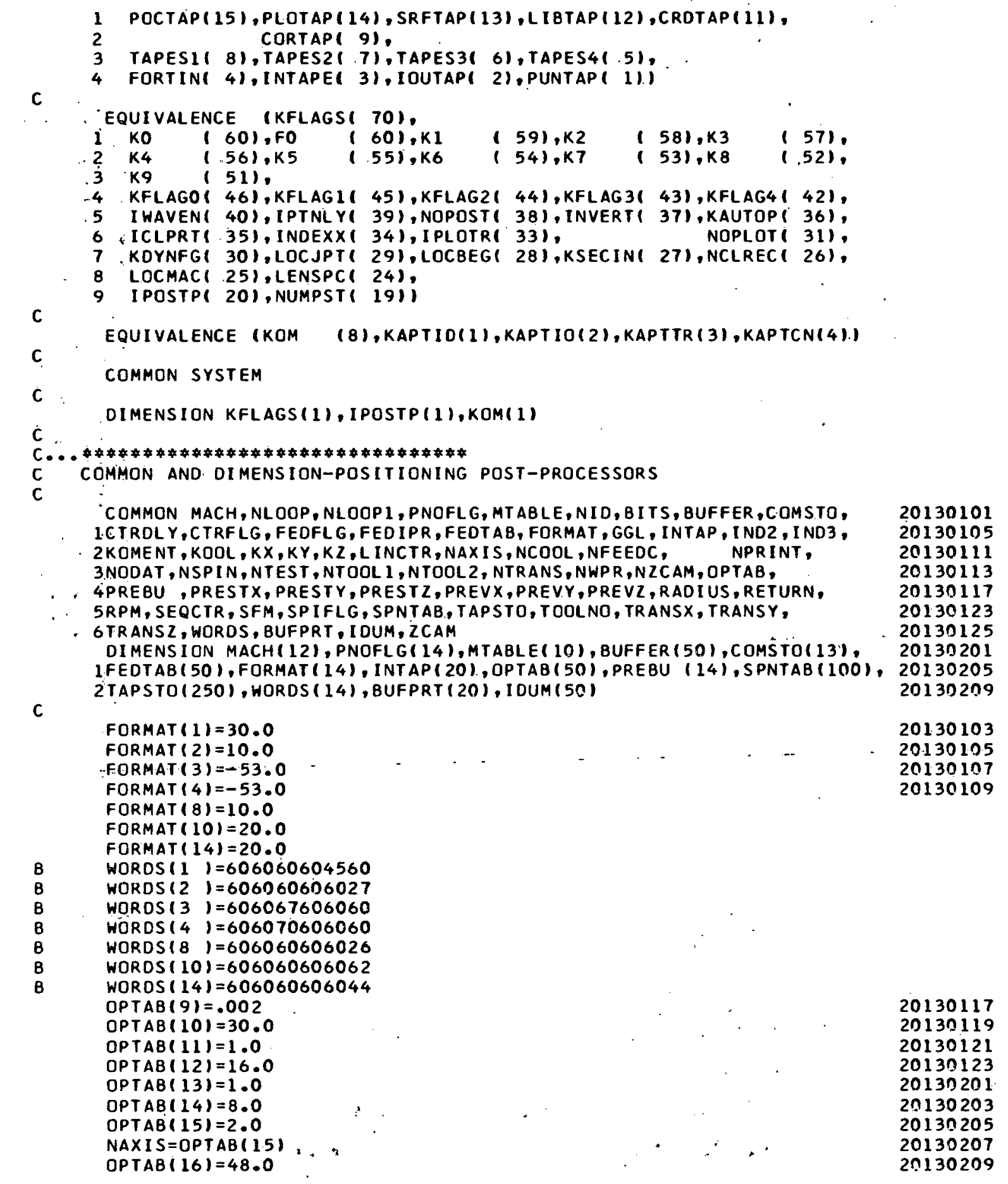




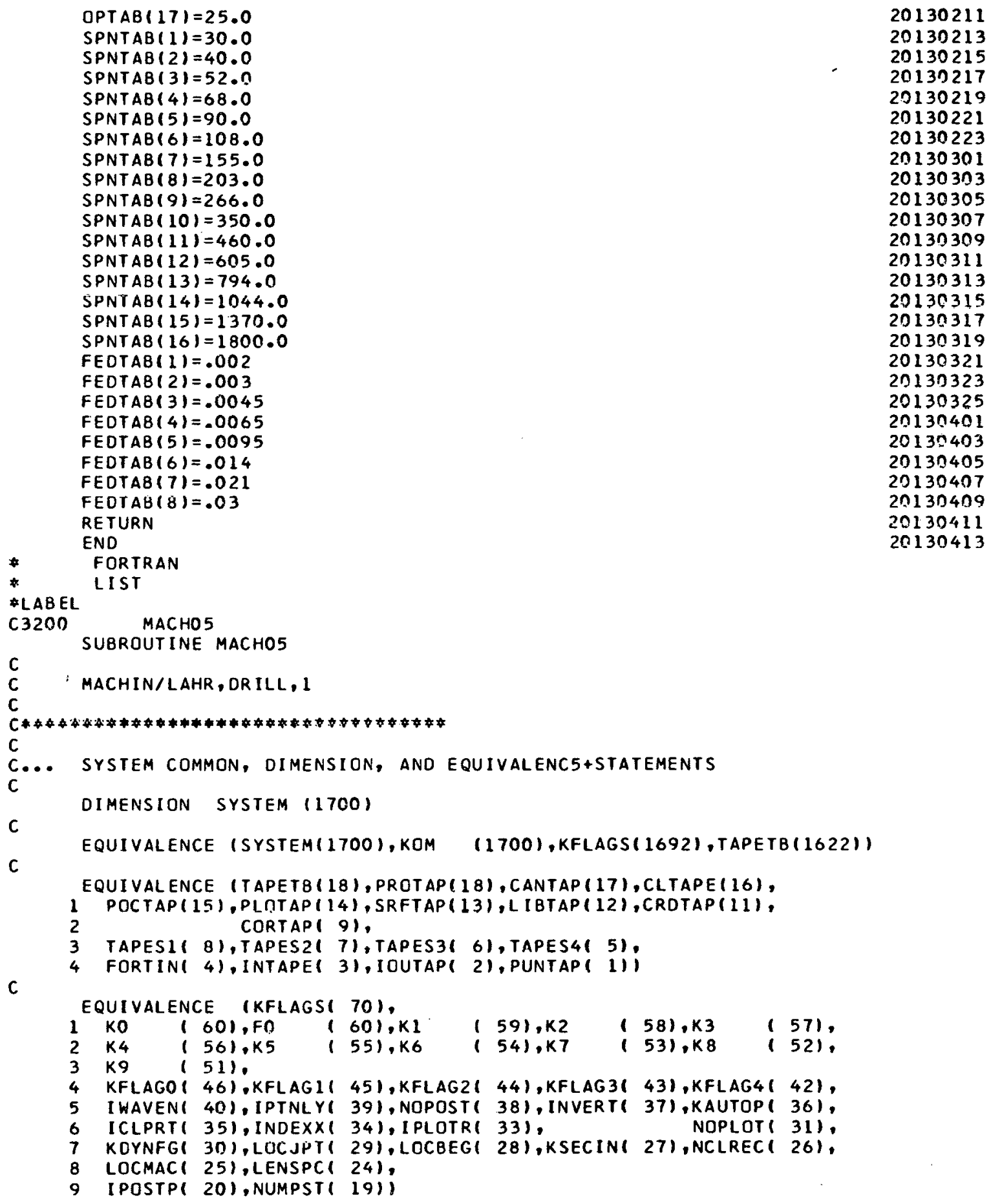


C

EQUIVALENCE (KOM (8),KAPTIO(1),KAPTIO(2),KAPTTR(3), KAPTCN(4))

COMMON SYSTEM

C

DIMENSION KFLAGS $(1)$, IPOSTP(1),KOMI 1$\}$

C

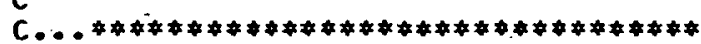

C. COMMON AND DIMENSION-POSITIONING POST-PROCESSORS

C

COMMON MACH, NLOOP, NLOOP 1, PNOFLG, MTABLE, NID, BITS, BUFFER, COMSTO, ICTRDL Y, CTRFLG, FEDFLG, FEDIPR, FEDTAB, FORMAT, GGL, INTAP, IND2, IND3,

- 2KOMENT, KOOL, KX, KY, KZ, LINCTR,NAXIS, NCOOL, NFEEOC, NPRINT,

3NODAT, NSPIN, NTEST, NTOOL 1 , NTOOL 2 , NTRANS, NWPR, NZCAM, OPTAB,

4PREBU ,PRESTX, PRESTY, PRESTZ, PREVX, PREVY, PREVZ, RADIUS, RETURN,

5RPM, SEQCTR, SFM, SP IFLG, SPNTAB, TAPSTO, TOOLNO, TRANSX, TRANSY,

GTRANSZ, WORDS, BUFPRT, I DUM, ZCAM

: DIMENSION MACH(12), PNOFLG (14), MTABLE(10), BUFFER (50),COMSTO(13),

IFEDTAB (50), FORMAT ( 14), INTAP (20), OPTAB (50), PREBU $(14)$, SPNTAB $(100)$,

2TAPSTO(250), WORDS (14), BUFPRT (20), I DUM(50)

FORMAT $(1)=30.0$

F.ORMAT $(3)=64.0$

FORMAT $(4)=64.0$

B WORDS (1) $=606060604560$

WORDS( 3$)=606760606060$

WORDS( 4$)=607060606060$

C

$O P T A B(15)=2.0$

$O P T A B(16)=50.0$

OPTAB $(17)=50.0$

NAXIS $=2.0$

RETURN

END

* FORTRAN

* LIST.

* $\angle A B E L$

C3300 MACHOG

SUBROUTINE MACHOG

C MACHIN/AMERCN, TABLE, 1

C MACHIN/KNIGHT, JIGBOR,I

C

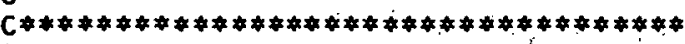

C

C... SYSTEM COMMON, DIMENSION, AND EQUIVALENC5+STATEMENTS

C

C

OIMENSION SYSTEM 117001

C

EQUIVALENCE (SYSTEM(1700),KOM (1700),KFLAGS(1692), TAPETB(1622))

EQUIVALENCE (TAPETB(18), PROTAP(18), CANTAP (17),CLTAPE(16),

1 POCTAP (15), PLOTAP (14), SRFTAP(13), L IBTAP (12), CRDTAP $(11)$,

2 CORTAP( 9$).$

3 TAPES1( 81 ,TAPES $2(7)$,TAPES31 6), TAPES41 51 ,

C FORTIN( 4), INTAPE( 3), IOUTAP( 2),PUNTAPI 11)

equivalence IKflagsi 70 ), 
$\mathrm{C}$

C

C

C

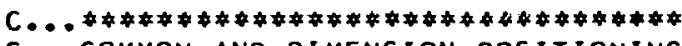

C COMMON AND DIMENSION-POSITIONING POST-PROCESSORS

COMMON MACH, NLOOP, NLOOP I, PNOFLG, MTABLE, NID, BITS, BUFFER, COMSTO, ICTRDL Y, CTRF LG, FEDFLG, FED IPR, FEDTAB, FORMAT, GGL, INTAP, I ND2, IND3, 2 KOMENT, KOOL, KX,KY,KZ,LINCTR, NAXIS, NCOOL, NFEEDC, NPRINT, 3 NODAT, NSP I N, NTEST, NTOOL 1 , NTOOL2, NTRANS, NWPR, NZCAM, OPTAB, 4PREBU , PRESTX, PRESTY, PREST Z, PREVX, PREVY, PREVZ, RADIUS, RETURN, SRPM, SEQCTR, SFM, SPIFLG, SPNTAB, TAPSTO, TOOLNO, TRANSX, TRANSY, OTRANSZ, WORDS, BUFPRT, IDUM, ZCAM

DIMENS ION MACH (12), PNOFLG $(14)$, MTABLE ( 10$)$, BUFFER $(50), C O M S T O(13)$, IFEDTAB ( 50$)$, FORMAT ( 14), INTAP (20), OPTAB (50), PREBU (14), SPNTAB(100),

C 2TAPSTO(250), HORDS $(14)$, BUFPRT $(20)$, IOUM(50)

DIMENSION HELP(4)

FORMAT $(3)=64.0$

FORMAT $(4)=64.0$

FORMAT $(14)=64.0$

WORDS( $3 \quad 1=606760606060$

WORDS $(4)=607060606060$

$\operatorname{WORDS}(14)=606044606060$

$O P T A B(1)=1.0$

$O P T A B(15)=2.0$

$O P T A B(16)=50.0$

$O P T A B(17)=50.0$

$O P T A B(20)=1.0$

$\operatorname{IDUM}(25)=1$

NAXIS $=2$

$D 02, J=1,3$

8

$2 \operatorname{HELP}(J)=000000000000$

HELP( 4$)=536060606060$

D05 $M=1,3$

$N=-24$

$J=0$

$\mathrm{K}=\mathrm{O}$

B

5 CALL PUNCHA (N,HELP,J,K)

HELP $(3)=040000000000$

CALL PUNCHA $(N, H E L P, J, K)$

NTOOL $1=0$

RETURN

END

FORTRAN
20130101 20130105 20130111 20130113 20130117 20130123 20130125 20130201

20130205 20130209 


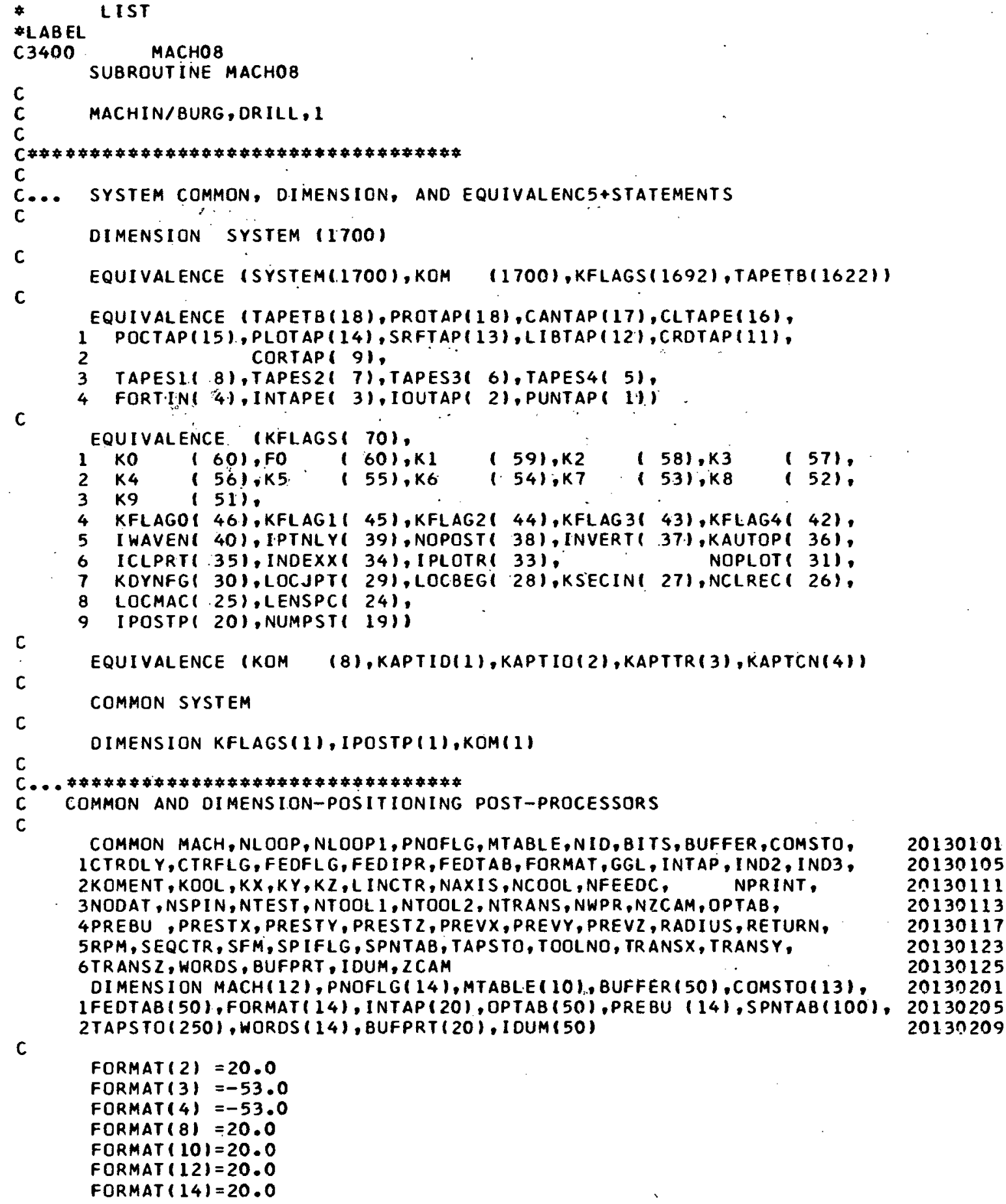

COMMON MACH, NLOOP, NLOOPI, PNOFLG, MTABLE, NID, BITS, BUFFER, COMSTO, ICTRDLY, CTRFLG, FEDFLG, FED IPR , FEDTAB, FORMAT, GGL, INTAP, IND2, IND3, 2KOMENT, KOOL, KX, KY, KZ, LINCTR, NAXIS, NCOOL, NFEEOC, NPRINT, 3NODAT, NSPIN, NTEST, NTOOL 1 , NTOOL2, NTRANS, NWPR, NZCAM, OPTAB, 4PREBU , PRESTX, PRESTY, PREST Z, PREVX, PREVY, PREVZ, RADIUS, RETURN, 5RPM, SEQCTR, SFM, SP IFLG, SPNTAB, TAPSTO, TOOLNO, TRANSX, TRANSY, 6TRANSZ, WORDS, BUFPRT, IDUM, ZCAM

DIMENS I ON MACH 12 , , PNOFLG (14), MTABLE (10), BUFFER(50), COMSTO (13), 1FEDTAB (50), FORMAT ( 14), INTAP (20), OPTAB (50), PREBU $(14)$, SPNTAB $(100)$, 2TAPSTO(250), WORDS $(14)$, BUFPRT $(20)$, I DUM $(50)$

20130101 20130105 20130111 20130113 20130117 20130123 20130125 20130201 20130205 20130209 


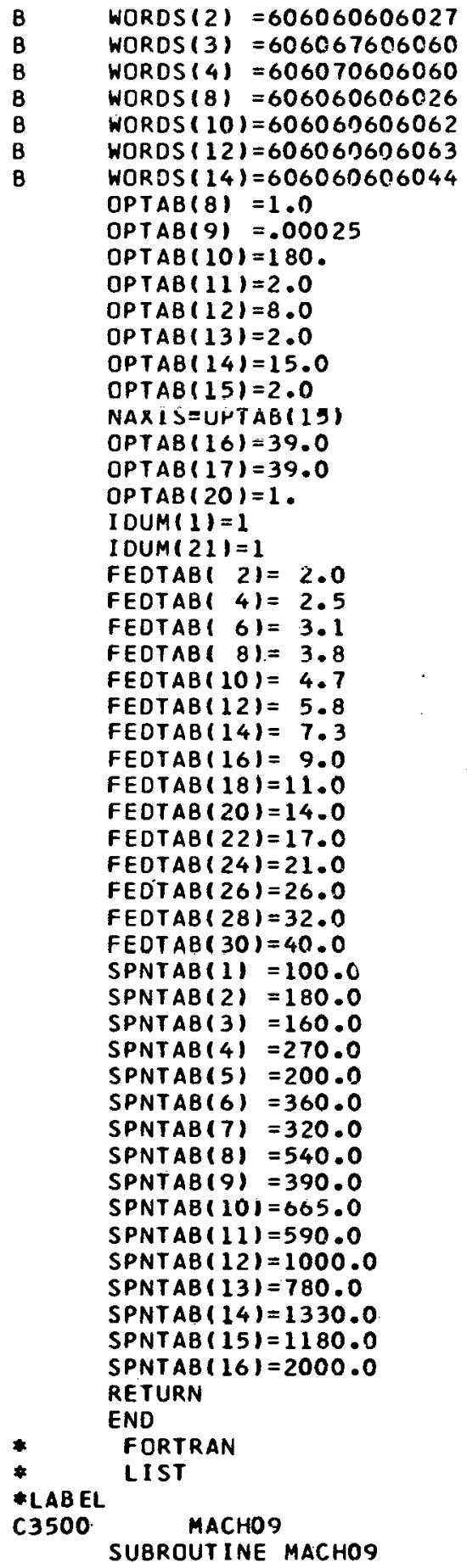




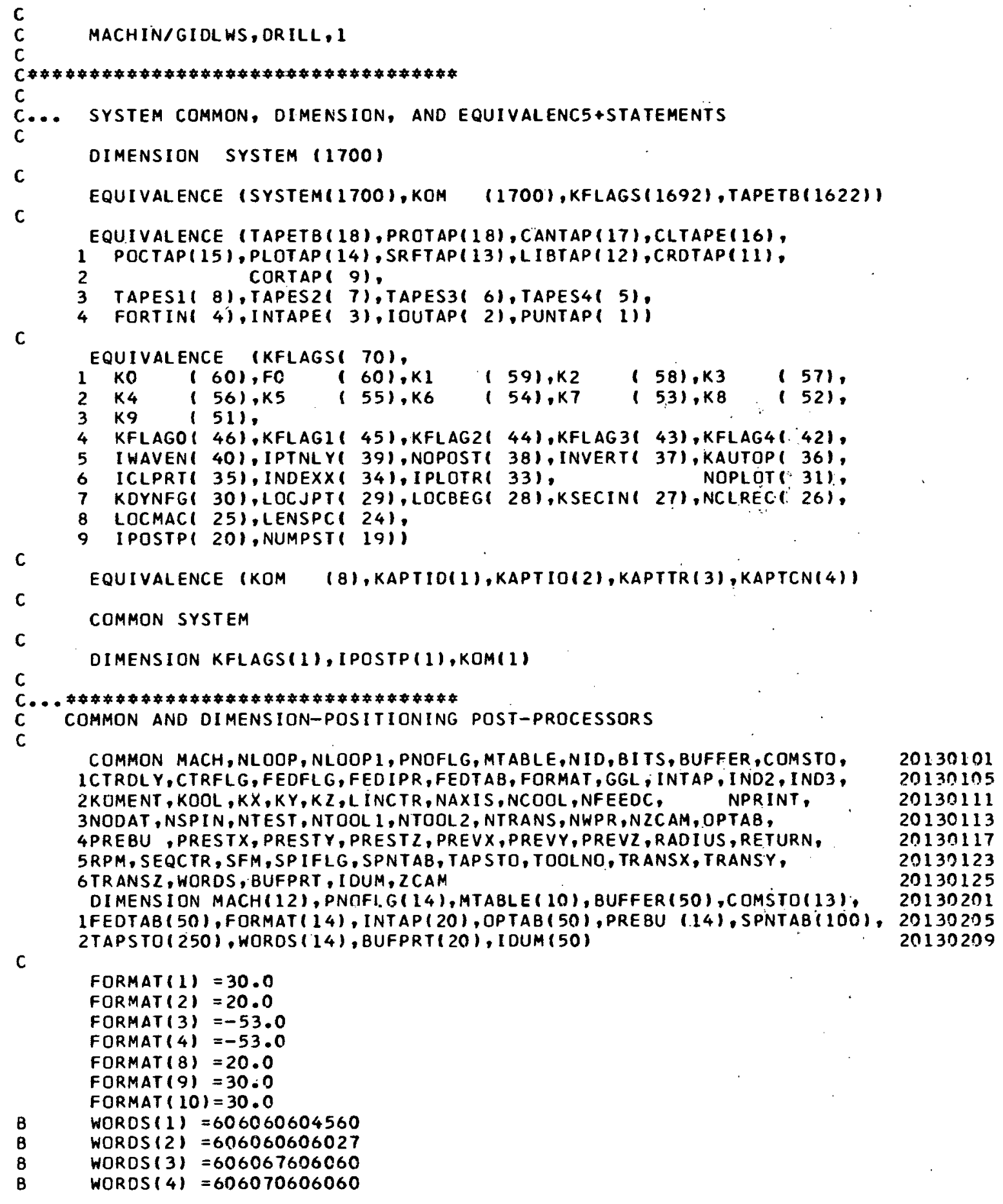




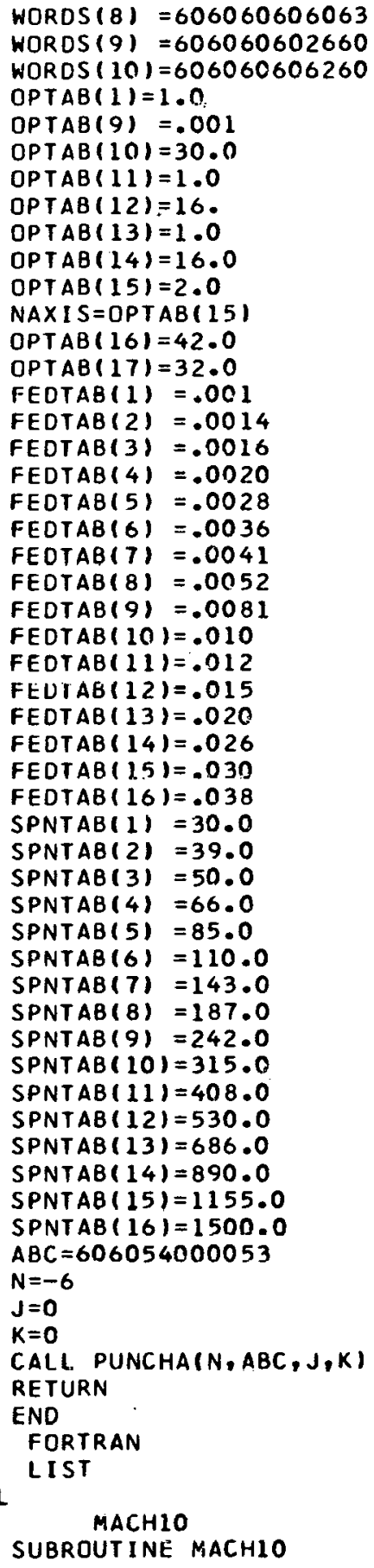




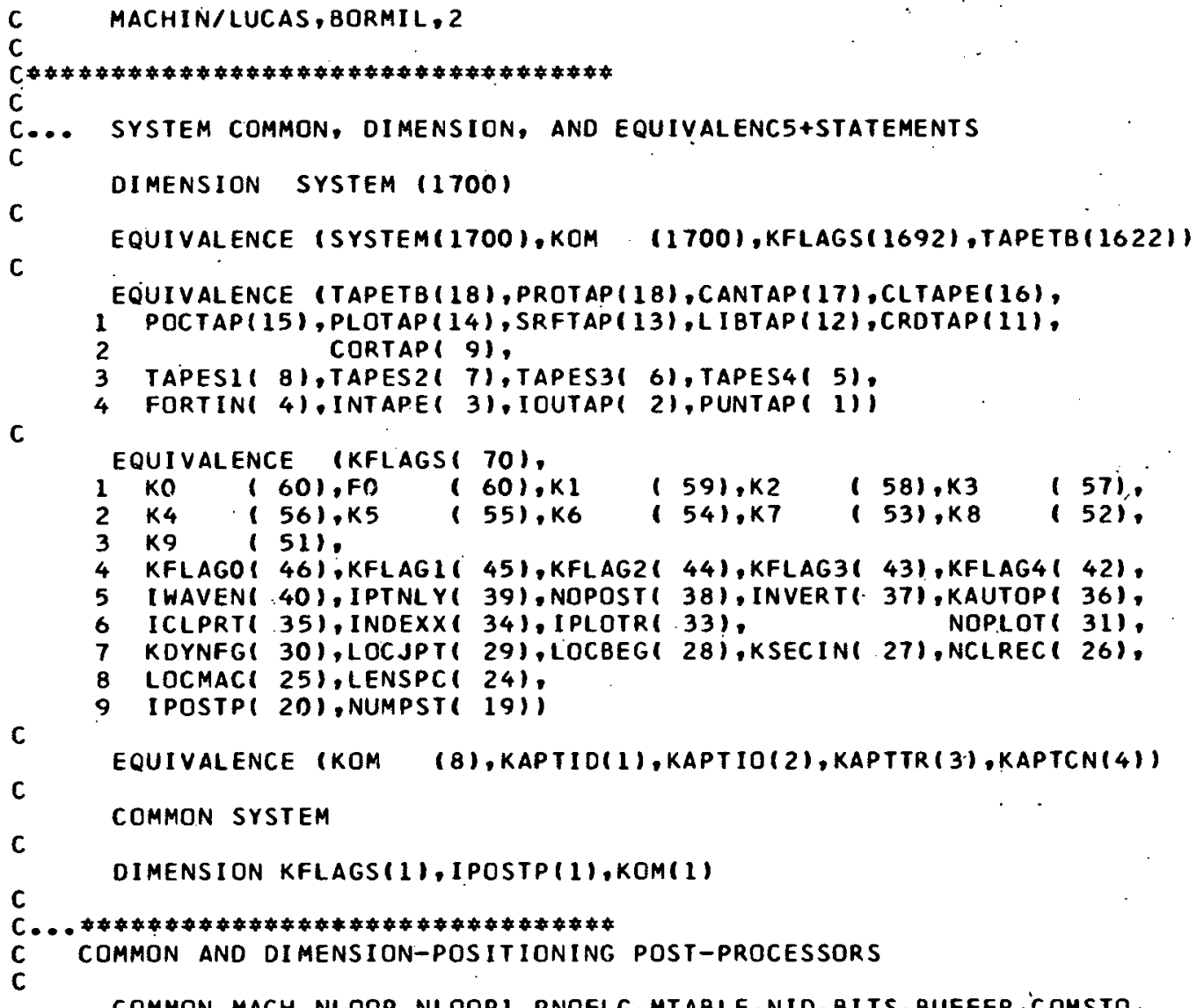

COMMON MACH, NLOOP, NLOOP1, PNOFLG, MTABLE,NID, BITS,BUFFER,COMSTO, 20130101 ICTRDL $Y$, CTRFLG, FEDFL G, FEDIPR, FEDTAB, FORMAT, GGL, INTAP, IND2, IND3, . 20130105 2KOMENT, KOOL, KX,KY,KZ,LINCTR, NAXIS,NCOOL, NFEEOC, NPRINT, 20130111 3NODAT, NSP IN, NTEST, NT OOL 1, NTOOL2, NTRANS, NWPR, NZCAM, OPTAB, . $\quad .20130113$ 4PREBU, PRESTX, PRESTY,PRESTZ, PREVX,PREVY,PREVZ,RADIUS, RETURN, 20130117 5RPM, SEQCTR, SFM, SPIFLG, SPNTAB, TAPSTO, TOOLNO, TRANSX, TRANSY, $\quad 20132123$ GTRANSZ, WORDS, BUFPRT, I DUM, ZCAM 20130125

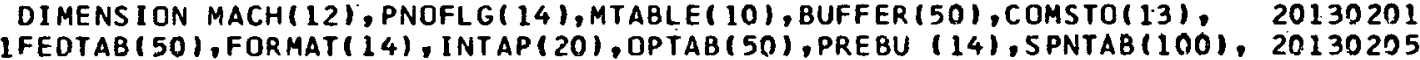

C 2TAPSTO (250), HORDS $(14)$, BUFPRT $(20)$, IOUM(50) 


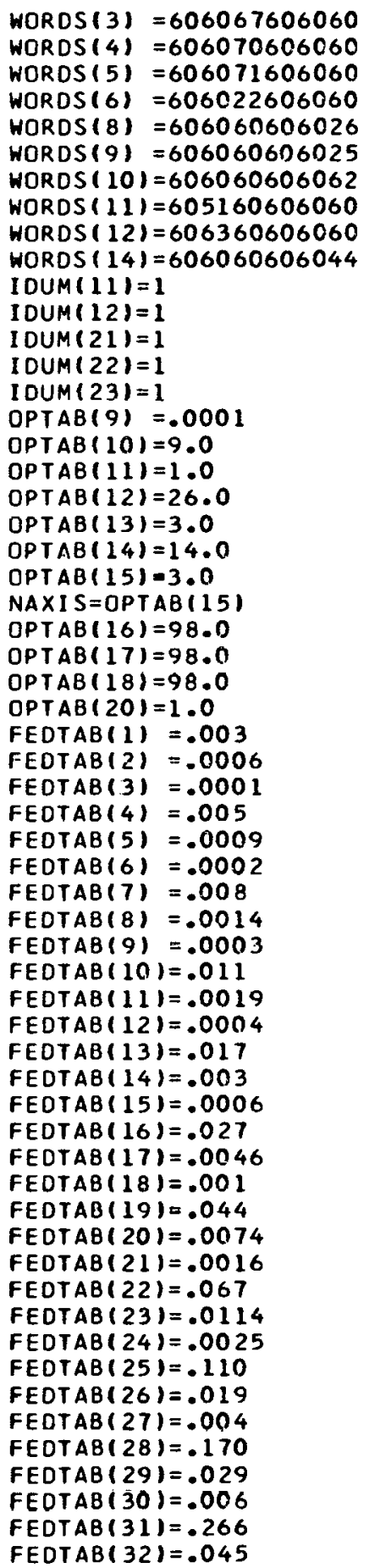




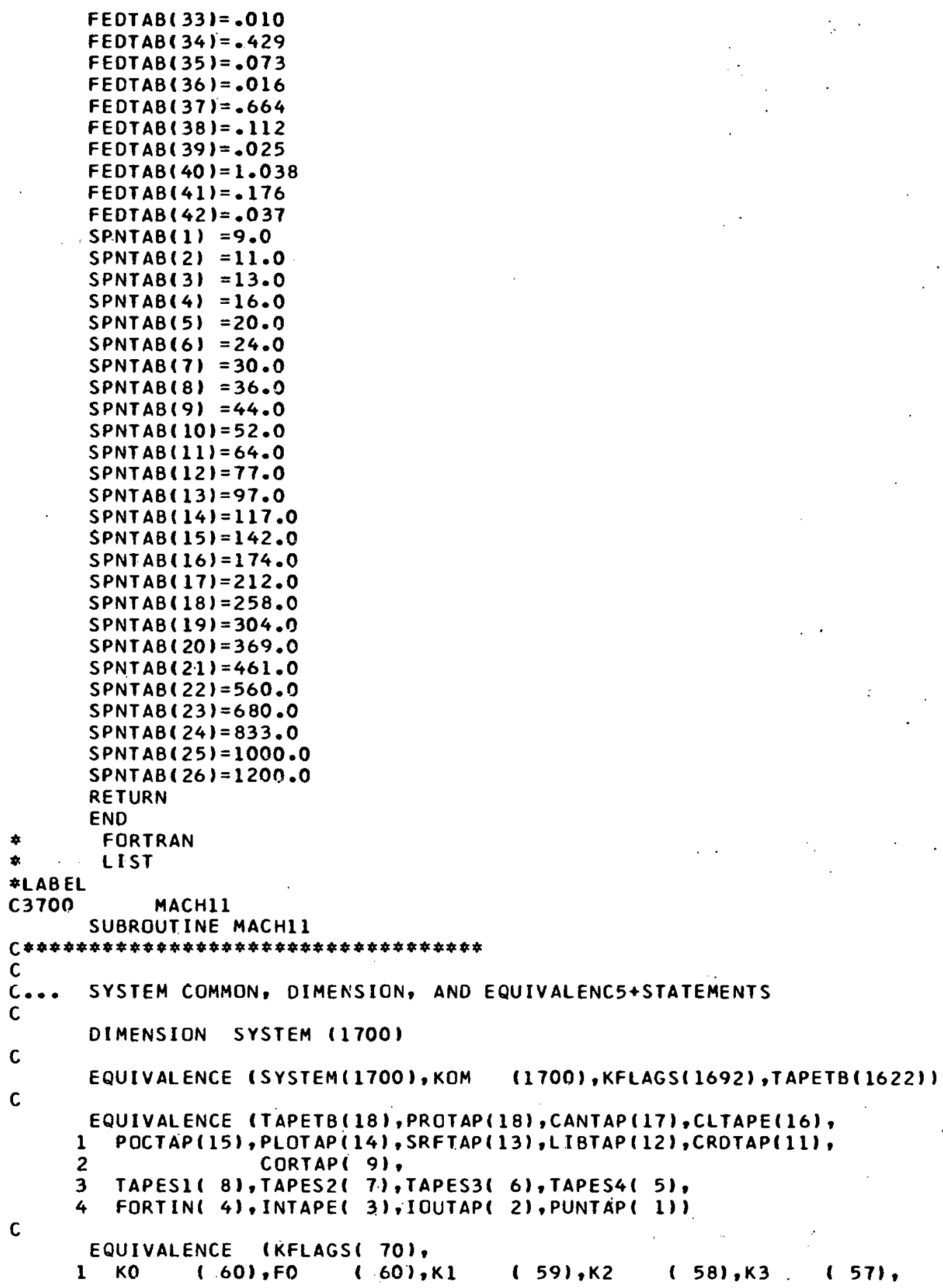




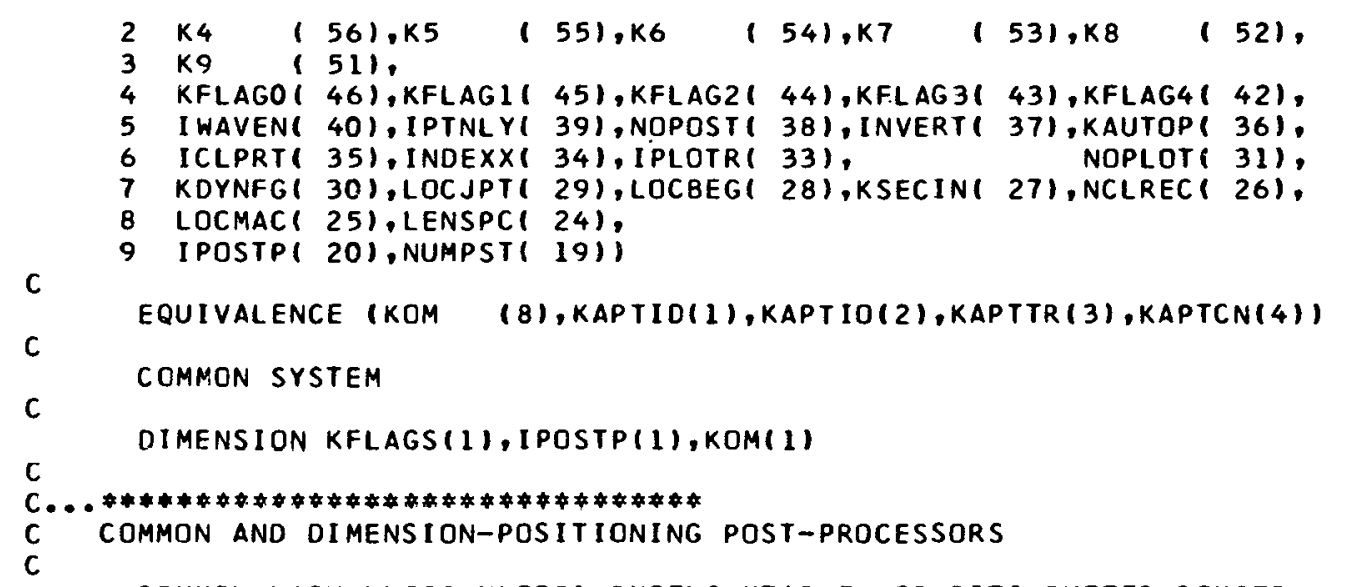

COMMON MACH, NLOOP, NLOOPI, PNOFLG, MTABLE,NID,BITS, BUFFER, COMSTO, ICTROLY, CTRFLG, FEDFL G, FED IPR, FEDTAB, FORMAT, GGL, INTAP, IND2, IND3, 2KOMENT, KOOL, KX, KY, KZ, LINCTR, NAXIS, NCOOL, NFEEDC, NPRINT, 3NODAT, NSPIN, NTEST, NTOOL 1, NTOOL2, NTRANS, NWPR , NZCAM, OPTAB, 4PREBU ,PRESTX, PRESTY, PRESTZ, PREVX,PREVY, PREVZ, RADIUS, RETURN, 5RPM. SEQCTR, SFM, SP I FLG, SPNTAB, TAPSTO, TOOLNO, TRANSX, TRANSY, 6TRANSZ, WORDS, BUFPRT, IDUM, ZCAM

DIMENSION MACH (12), PNOFLG 14 ), MTABLE( 10$)$, BUFFER (50), COMSTO(13), 20130105 20130111 20130113 20130117 20130123 20130125 IFEDTAB (50) FORMAT (14), INTAP (20), OPTAB (50) OPREBU $(14)$ OSPNTAB $(100), 20130205$

C 2TAPSTO $(250)$, WORDS $(14)$, BUFPRT $(20)$, IDUM(50)

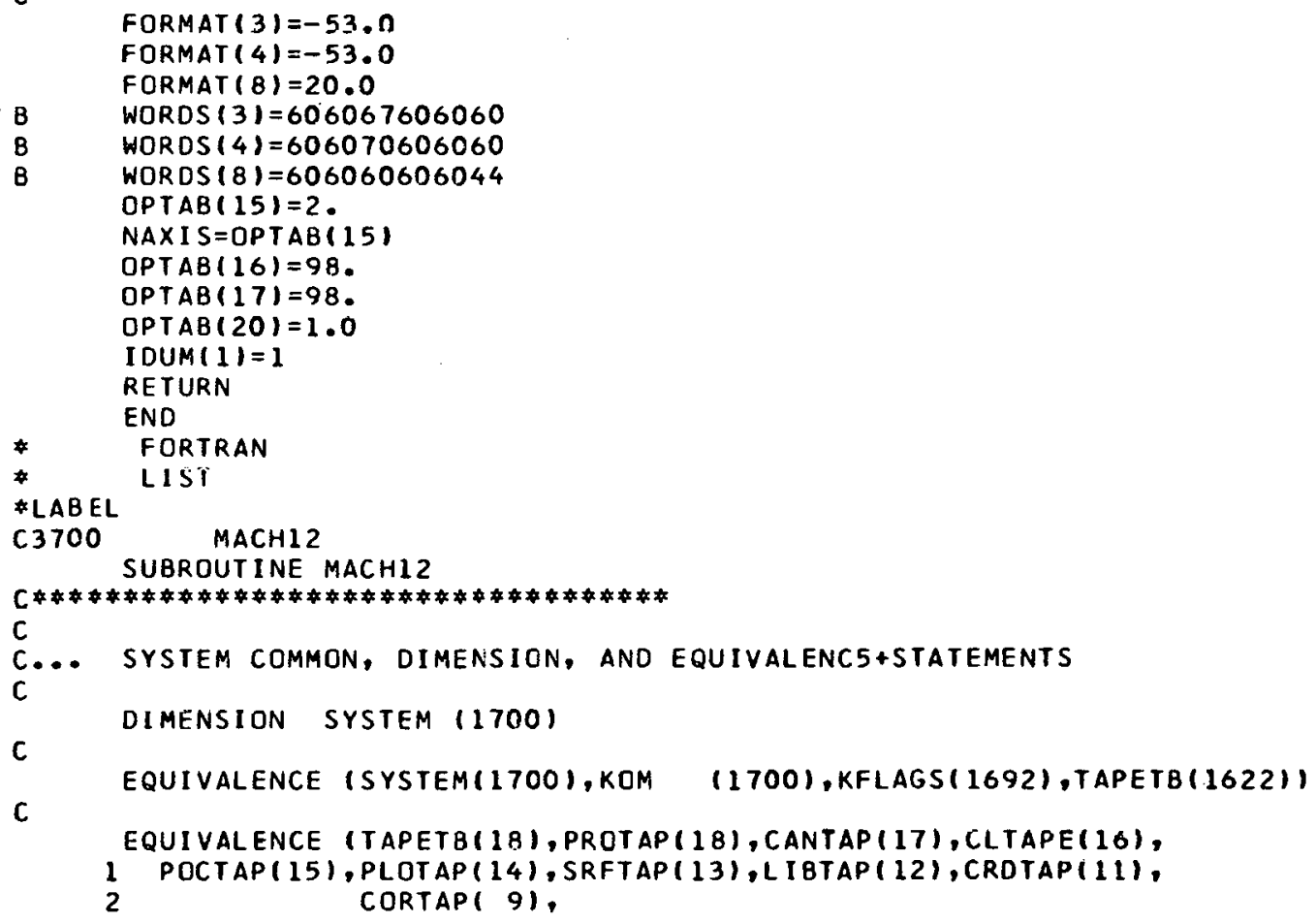




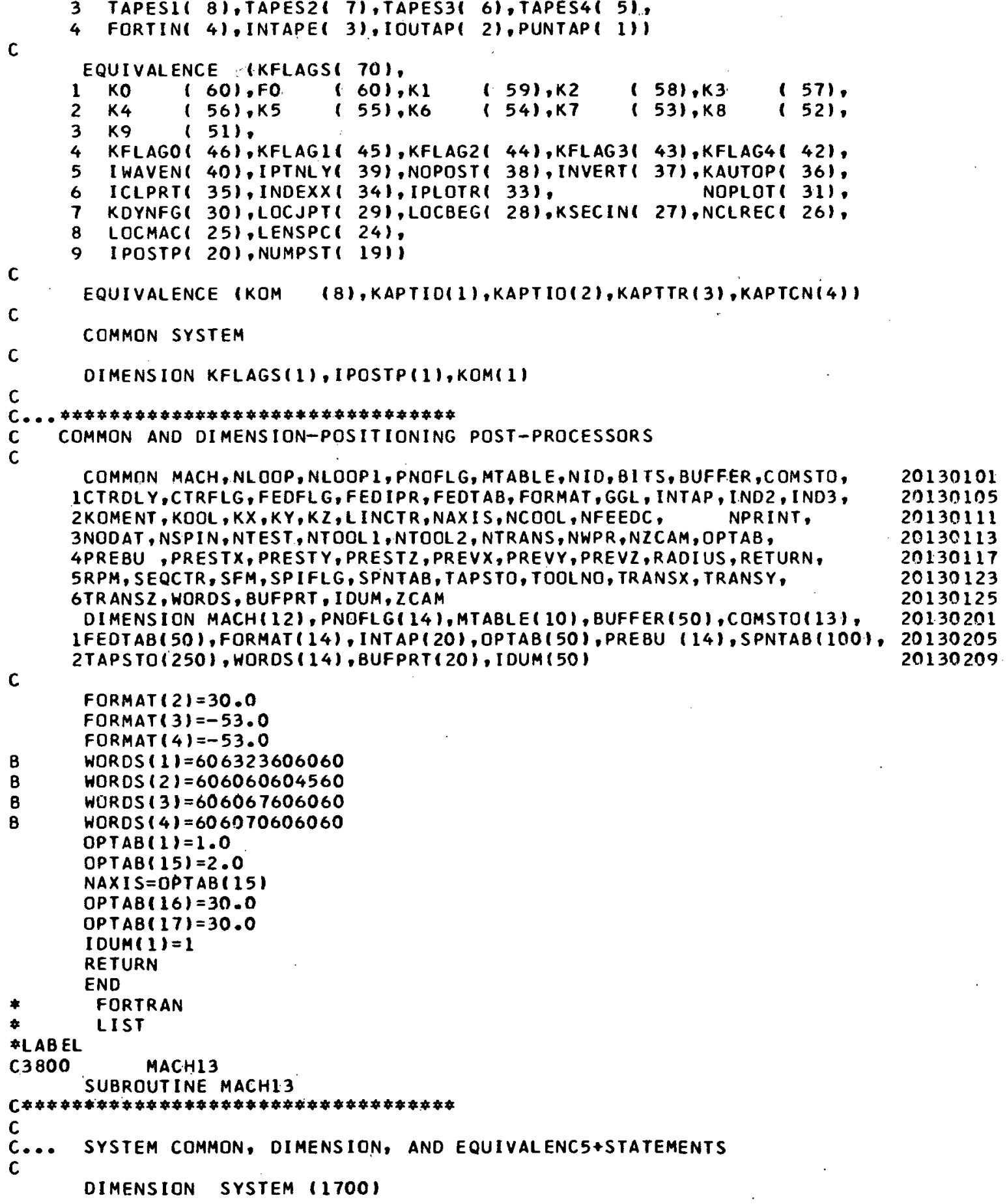


C

C EQUIVALENCE (SYSTEM(1700),KOM (1700),KFLAGS(1692), TAPETB(1622))

EQUIVALENCE (TAPETB(18),PROTAP(18), CANTAP $(17), \operatorname{CLTAPE}(16)$,

1 POCTAP (15), PLOTAP (14), SRFTAP (13), LIBTAP (12),CRDTAP(11).

2 CORTAP $(9)$,

3 TAPES1 $(8)$, TAPES $2(7)$,TAPES3 3 , ,TAPES $4(5)$,

C

4 FORTIN( 4), INTAPE( 31 , IOUTAP( 2), PUNTAP( 1)

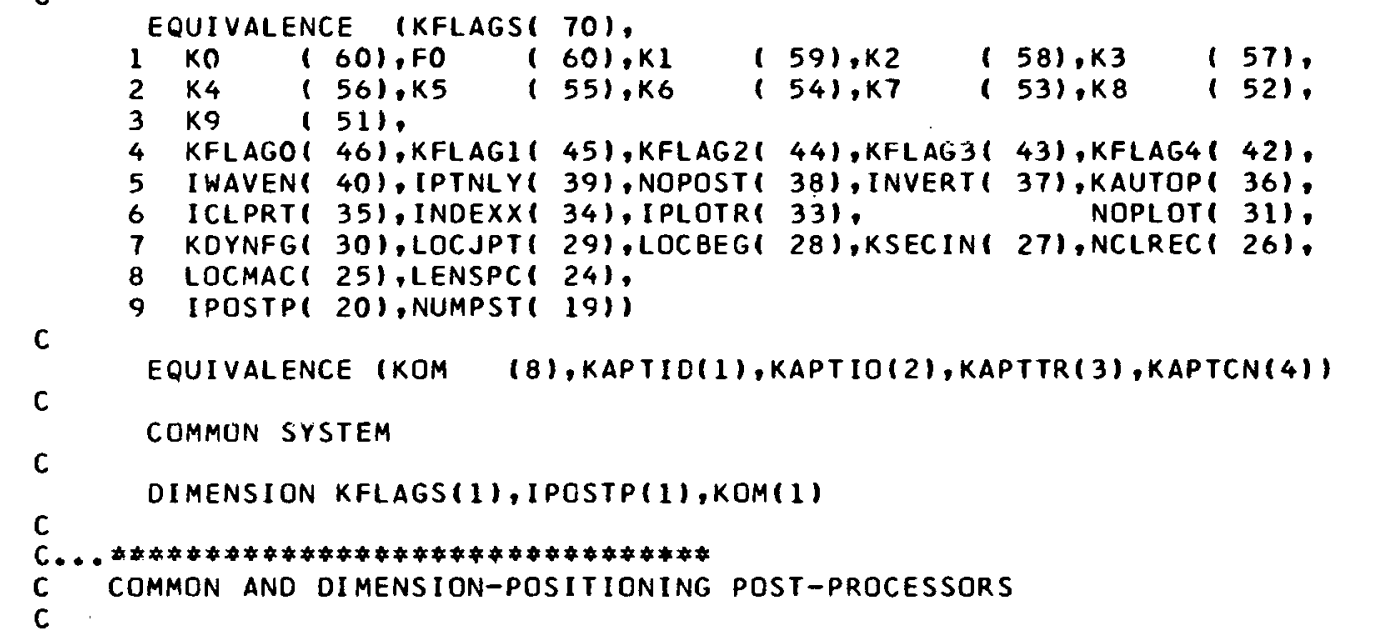

COMMON MACH, NLOOP, NLOOP1, PNOFLG, MTABLE, NID, BITS, BUFFER, COMSTO, 20130101 ICTRDLY, CTRFLG, FEDFL G, FEOIPR, FEOTAB, FORMAT, GGL, INTAP, IND2, IND3, $2 K O M E N T, K O O L, K X, K Y, K Z, L$ INCTR, NAXIS, NCOOL, NFEEDC, NPRINT, 3NODAT, NSPIN, NTEST, NTOOL 1 , NTOOL 2, NTRANS, NWPR, NZCAM, OPTAB, 4PREBU, PRESTX, PRESTY, PRESTZ, PREVX, PREVY, PREVZ, RAOIUS, RETURN, 5RPM, SEQCTR, SFM, SP I FLG, SPNT AB, TAPSTO, TOOLNO, TR ANSX, TRANSY, 6TRANSZ, WORDS, BUFPRT, IDUM, ZCAM

DIMENS ION MACH $(12)$, PNOFLG $(14)$, MTABLE $(10), B U F F E R(50), C O M S T O(13)$, IFEDTAB (50), FORMAT (14), INTAP (20), OPTAB (50), PREBU (14), SPNTAB $(100)$,

C 2TAPSTO $(250)$, WORDS $(14)$, BUFPRT $(20)$, I DUM(50)

FORMAT $(1)=30.0$

FORMAT $(3)=-53.0$

FORMAT $(4)=-53.0$

FORMAT $(5)=-53.0$

FORMAT $(8)=30.0$

FOP.MAT $(10)=20.0$

FURMAT $(14)=20.0$

$\operatorname{WORDS}(1)=606060604560$

WOROS $(3)=606067606060$

WORDS $(4)=606070606060$

WOROS $(5)=606071606060$

WOROS $(8)=606060606026$

WORDS $(10)=606060606062$

45720319

45720320

45720321

45720322

45720323

45720324

45720325

45720401

45720402

45720403

45720404

45720405

45720406

45720407

UPTAB $(8)=0$.

45720409

OPTAB $(9)=70$

OPTAB $(11)=1$.

OPTAB (12) $=32$.

45720410

45720411

OPTAB $(15)=3$. 
$N A X I S=O P T A B(15)$

45720413

OPTAB $(16)=99$.

$O P T A B(17)=99$. OPTAB $(18)=99$. OPTAB $(20)=0$. $O P T A B(29)=9.0$ $\operatorname{DUM}(21)=1$ $\operatorname{IDUM}(23)=1$ $\operatorname{IDUM}(28)=1$

SPNTAB $(1)=100$

SPNTAB (2) $=111$

SPNTAB (3) $=125$

SPNTAB $(4)=138$

SPNTAB $(5)=153$

SPNTAB $(6)=169$

SPNTAB $(7)=190$

SPNTAB $(8)=210$

SPNTAB $(9)=253$

SPNTAB $(10)=279$

SPNTAB $(11)=315$

SPNTAB $(12)=347$

SPNTAB $(13)=385$

SPNTAB $(14)=423$

SPNTAB (15) $=478$

SPNTAB(16) $=528$

SPNTAB (17) $=564$

SPNTAB $(18)=620$

SPNTAB $(19)=700$

SPNTAB $\left(20^{\circ}\right)=772$

SPNTAB $(21)=857$

SPNTAB $(22)=944$

SPNTAB (23) $=1064$

SPNTAB (24) $=1178$

SPNTAB $(25)=1420$

SPNTAB $(26)=1562$

SPNTAB $(27)=1764$

SPNTAB $(28)=1942$

SPNTAB $(29)=2160$

SPNTAB $(30)=2378$

SPNTAB $(31)=2682$

SPNTAB $(32)=2970$

RETURN

END

* Fortran

* L LIST

* LAB EL

SUBROUTINE MACHI 4

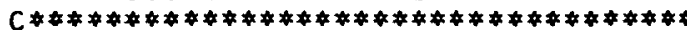

C

C... SYSTEM COMMON, DIMENSION, AND EQUIVALENC5+STATEMENTS

C

DIMENSION SYSTEM $(1700)$

$c$

C

EQUIVALENCE (SYSTEM(1700), KOM (1700),KFLAGS(1692), TAPETB(1622))

EQUIVALENCE (TAPETB(18), PROTAP(18), CANTAP (17), CLTAPE 16 ),

1 POCTAP (15), PLOTAP (14), SRFTAP 13$)$, LI BTAP $(12)$, CROTAP $(11)$,

2 CORTAPI 91,

3 TAPES 1181 , TAPES 21 7), TAPES3( 61, TAPES $4(5)$,

45720414

45720415

45720417

45720418

45720419

45720420

45720421

45720422

45720423

45720424

45720425

45720501

45720502

45720503

45720504

45720505

45720506

45720507

45720508

45720509

45720510

45720511

45720512

45720513

45720514

45720515

45720516

45720517

45720518

45720519

45720520

45720521

45720522

45720523

45720524 
4 FORTINI 4), INTAPEI 3), IOUTAPI 2),PUNTAP ( 111

C equivalence (KFlagsi 70$)$,

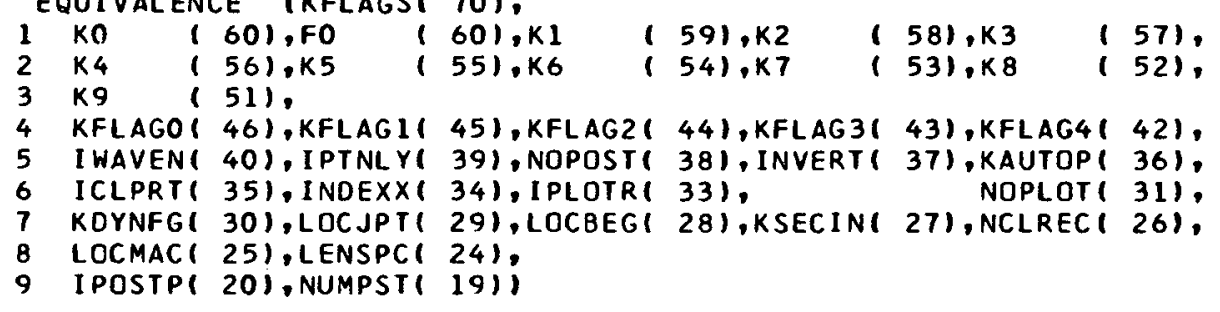

C

c

EQUIVALENCE (KOM (8),KAPTID(1), KAPTIO $(2), \operatorname{KAPTTR}(3), \operatorname{KAPTCN}(4))$

C

C

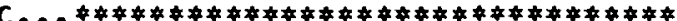

C

C

COMMON AND DIMENSION-POSITIONING POST-PROCESSORS

COMMON MACH, NLOOP, NLOOPL, PNOFLG, MTABLE, NID, BITS, BUFFER, COMSTO, ICTROL Y, CTRFLG, FEDFLG, FEDIPR, FEDTAB, FORMAT, GGL, INTAP, IND2, IND3, 2KOMENT, KOOL, KX,KY,KZ, L INCTR, NAXIS, NCOOL, NFEEOC, NPRINT, 3NODAT, NSPIN, NTEST, NTOOL 1 , NTOOL2, NTRANS, NWPR , NZCAM, OPTAB, 4PREBU, PRESTX, PRESTY, PRESTZ, PREVX, PREVY, PREVZ, RAOIUS, RETURN, 5RPM, SEQCTR, SFM, SP IFLG, SPNT AB, TAPSTO, TOOLNO, TR ANSX, TRANSY, 6TRANSZ, WORDS, BUFPRT, IDUM, ZCAM

OIMENSION MACH (12), PNOFLG (14), MTABLE (10), BUFFER (50), COMSTO (13), IFEDTAB (50), FORMAT (14), INTAP (20), OP TAB (50), PREBU (14), SPNTAB (100),

C 2TAPSTO(250), WORDS (14), BUFPRT (20), I DUM(50)

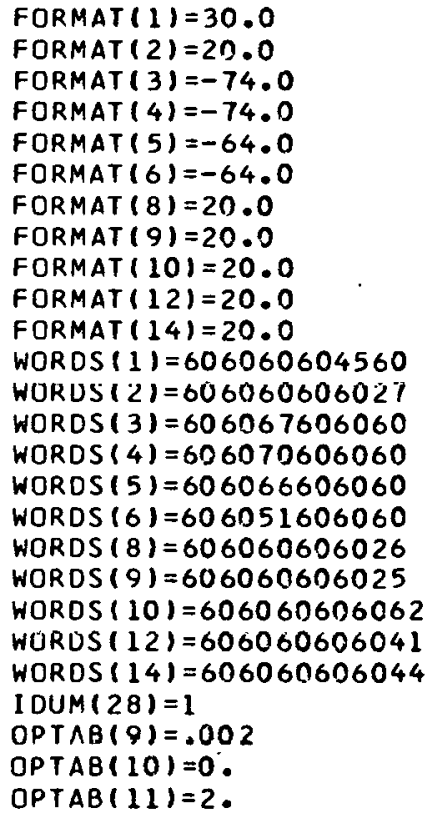




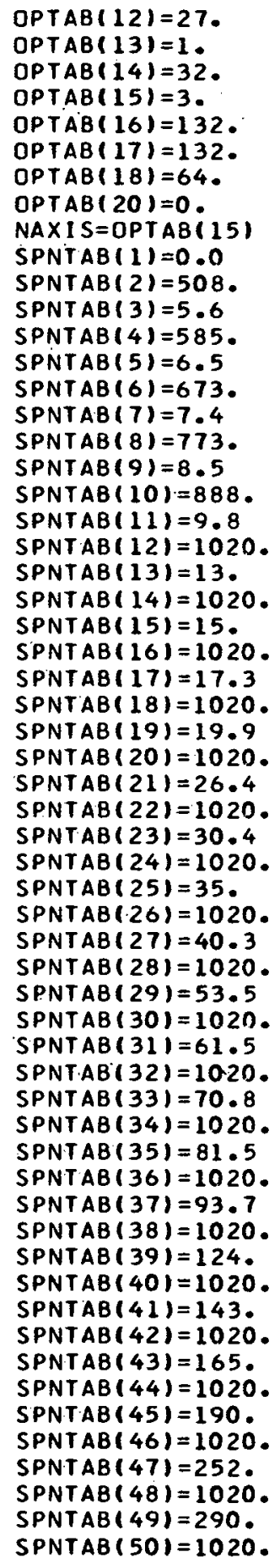


$\operatorname{SPNTAB}(51)=334$.

47490408

SPNTAB $(52)=1020$.

47490409

SPNTAB $(53)=384$.

SPNTAB $(54)=1020$.

47490410

FEDTAB $(1)=.012$

FEDTAB $(2)=.016$

FEDTAB $(3)=.021$

FEDTAB $(4)=.029$

FEOTAB $(5)=.039$

FEOTAB $(6)=.054$

FEDT $A B(7)=.070$

FEDTAB $(8)=.096$

FEDTAB $(9)=.130$

FEOTAB $(10)=.180$

FEDTAB $(11)=.230$

FEDTAB $(12)=.320$

FEOTAB $(13)=.420$

FEDTAB $(14)=.570$

FEDTAB (15) $=.750$

$\operatorname{FEDTAB}(16)=1.000$

FEDTAB $(17)=1.4$

FEOTAB $(18)=1.9$

FEDTAB $(19)=2.5$

FEOTAB $(20)=3.4$

FEDT $A B(21)=4.5$

FEDTAB $(22)=6.2$

FEDTAB $(23)=8.1$

FEOTAB $(24)=11$.

FEDTAB $(25)=15$.

FEDTAB $(26)=20$.

FEDTAB $(27)=27$.

FEDTAB $(28)=36$.

FEDTAB $(29)=49$.

FEDTAB $(30)=67$.

FEDTAB $(31)=87$.

FEOTAB $(32)=120$.

RETURN

END

47490411

47490412

47490413

47490414

47490415

47490416

47490417

47490418

47490419

47490420

47490421

47490422

47490423

47490424

47490425

47490501

47490502

47490503

47490504

47490505

47490506

47490507

47490508

47490509

47490510

47490511

47490512

47490513

47490514

47490515

47490516

47490517

47490518

47490519

$4749052 n$

* FORTRAN

* LIST

\# $\mathrm{ABEL}$

C4100 MESSAG

SUBROUTINE MESSAG

45720810

45720809

C

C

C... SYSTEM COMMON, DIMENSION, AND EQUIVALENC5 +STATEMENTS

C

C

DIMENSION SYSTEM $(1700)$

C EQUIVALENCE (SYSTEM(1700), KOM (1700),KFLAGS(1692), TAPETB(1622))

EQUIVALENCE (TAPETB(18), PROTAP $(18), \operatorname{CANTAP}(17), \mathrm{CLTAPE}(16)$,

1 POCTAP (15), PLOTAP (14), SRFTAP (13), L IBTAP (12), CRDTAP (11), CORTAPI 91 ,

TAPES1( 8$)$, TAPES $2(7)$, TAPES31 6), TAPES4( 51 ,

FORTIN( 4 ). INTAPE ( 3), IOUTAPI 2), PUNTAP( 11 )

C

EQUIVALENCE (KFLAGS( 70$)$.
1 KO
$(601, F O$
( 60$), K 1$
$1591, \mathrm{~K} 2$
$(58), K 3$
(57), 


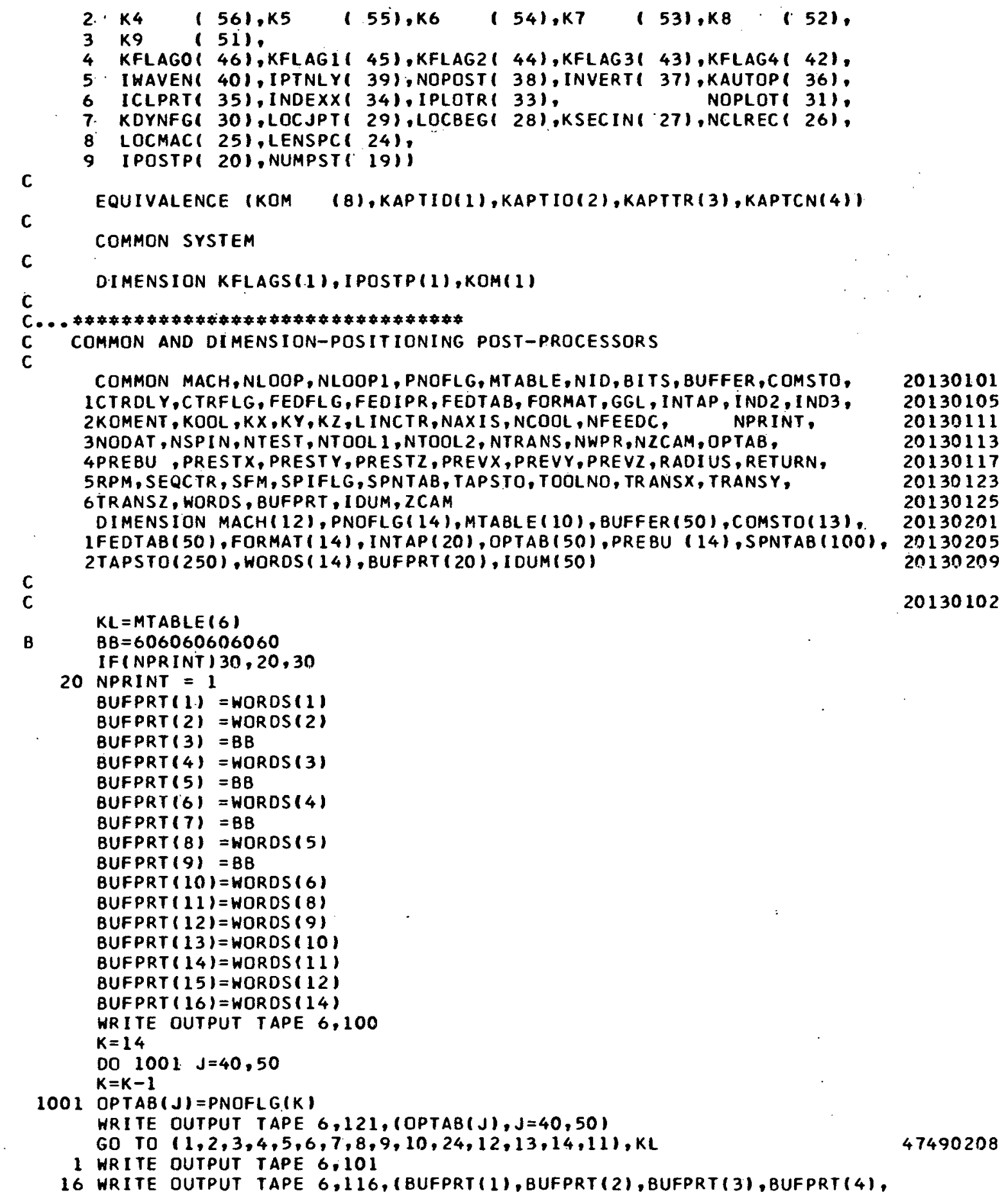


1BUFPRT (5), BUFPRT (6), BUFPRT (7), BUFPRT (8), BUFPRT(11), BUFPRT (13), 2BUFPRT (16))

GO TO 11

2 HRI TE OUTPUT TAPE 6.102 GO TO 16

3 WRITE DUTPUT TAPE 6,103 GO TO 16

4 WRITE OUTPUT TAPE 6,104 GO TO 16

5 WRITE OUTPUT TAPE 6,105 GO TO 16

6 WRITE OUTPUT TAPE 6,106

17 WRITE OUTPUT TAPE $6,117,1$ BUFPRT (2), BUFPRT (3), BUFPRT (4), 1BUFPRT (5), BUFPRT (6), BUFPRT (7), BUFPRT ( 8), BUFPRT (11), BUFPRT (13), 2BUFPRT ( 15), BUFPRT (16)) GO TO 11

7 WRITE OUTPUT TAPE 6,107 GO TO 17

8 WRITE DUTPUT TAPE 6,108 GO TO 17

9 WRITE OUTPUT TAPE 6,109

23 WRITE DUTPUT TAPE $6,120,($ BUFPRT $(I), I=1,16)$ GO TO 11

10 WRITE OUTPUT TAPE 6,110 GO TO 23

24 WRITE OUTPUT TAPE 6,109 GO TO 17

12 WRITE OUTPUT TAPE 6,112 GO TO 23

13 WRITE OUTPUT TAPE 6,113 GO TO 16

14 WRITE OUTPUT TAPE 6,114 GO TO 23

11 LINCTR $=0$

30 IF (LINC TR-45) 18,20,20

18 LINCTR $=$ LINCTR+1 WRITE OUTPUT TAPE $6,1000,($ TAPSTŨ $(J), J=4,14)$

1000 FORMAT $(2 X, 11 A 6)$

100 FORMAT ( $1 \mathrm{H} 1)$

101 FORMAT $(26 H$ G. + L. POSITIONING TABLE///I

102 FORMAT (17H DEVLIEG JIG MILL///)

103 FORMAT (28H FOSDICK POSITIONING MACHINE///)

104 FORMAT ( $18 \mathrm{H}$ HEALD - HEALDRILL///)

105 FORMAT ( $13 \mathrm{H}$ LAHR - DRILL///)

106 FORMAT ( $27 H$ AMERICAN - DRILL///)

107 FORMAT (17H KNIGHT JIG BORER///)

108 FORMAT (I3H BURG - DRILL////

109 FORMAT $(14 \mathrm{H} \mathrm{G}$. + L. DRILL///I

110 FORMAT (18H LUCAS BORING MILL///)

112 FORMAT (20 H PRATT-WHITNEY ORILL///)

113 FORMAT (13H KRNTR-MCHCNT///)

114 FORMAT ( $15 \mathrm{H} \mathrm{G}$. + L. BORMIL///)

116 FORMAT $(11 \mathrm{AG} / / / /)$

117 FORMAT(6H N, $1146 / / / 1$

118 FORMAT (16A6)

119 FORMAT $(1 X, 15,1146)$

120 FORMAT $(16 A 6 / / /)$

121 FORMAT (1H, $11 A 6,53 \times)$ RETURN

45720107 45720108 47490269 47490210 


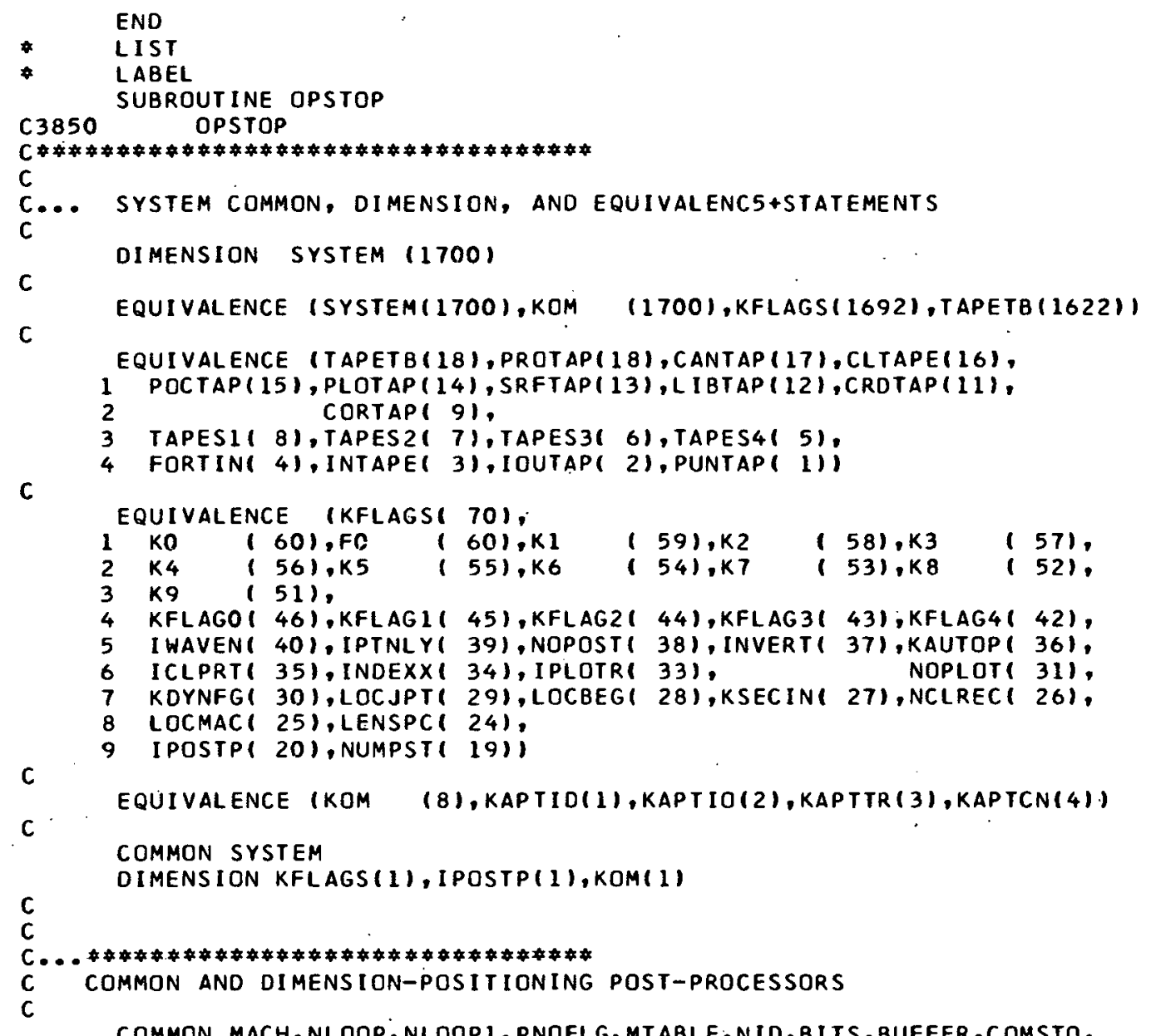

COMMON MACH, NLOOP, NLOOP1, PNOFLG, MTABLE, NID,BITS,BUFFER,COMSTO, 20130101 1CTROL Y, CTRFLG, FEDFLG, FEO IPR, FEDTAB, FORMAT, GGL, INTAP, IND2, IND3, . 20130105 2KOMENT, KOOL, KX,KY,KZ,LINCTR, NAXIS, NCOOL, NFEEDC, NPRINT, 3NODAT, NSPIN, NTEST, NTOOL 1 , NTOOL2, NTRANS, NHPR, NZCAM, OPTAB, 4PREBU, PRESTX, PRESTY, PRESTZ, PREVX, PREVY, PREVZ, RADIUS, RETURN, SRPM, SEQCTR, SFM, SPIFLG, SPNTAB, TAPSTO, TOOLNO, TRANSX, TRANSY, GTRANSZ, WORDS, BUFPRT, IDUM, ZCAM

DIMENS ION MACH $(12)$, PNOFLG $(14)$, MTABLE $(10)$, BUFFER $(50)$, COMSTO $(13)$, IFEDTAB (50), FORMAT ( 14), INTAP (20), OPTAB (50), PREBU $(14)$, SPNTAB (100); 2TAPSTO (250), WORDS $(14)$, BUFPRT $(20)$, IDUM(50)

C $N=M T A B L E(6)$ 20130209

If $(\mathrm{N}-15) 2,2,1$

2 Go To $(1,1,1,1,1,1,1,1,1,1,1,1,10,10,1), N$

10 PREQU $(14)=1$.

$S E Q C T R=S E Q C T R+O P T A B(22)$

PREBU ( I) = SEQCTR

CALL CARD

CALL PRINT

1 RETURN 


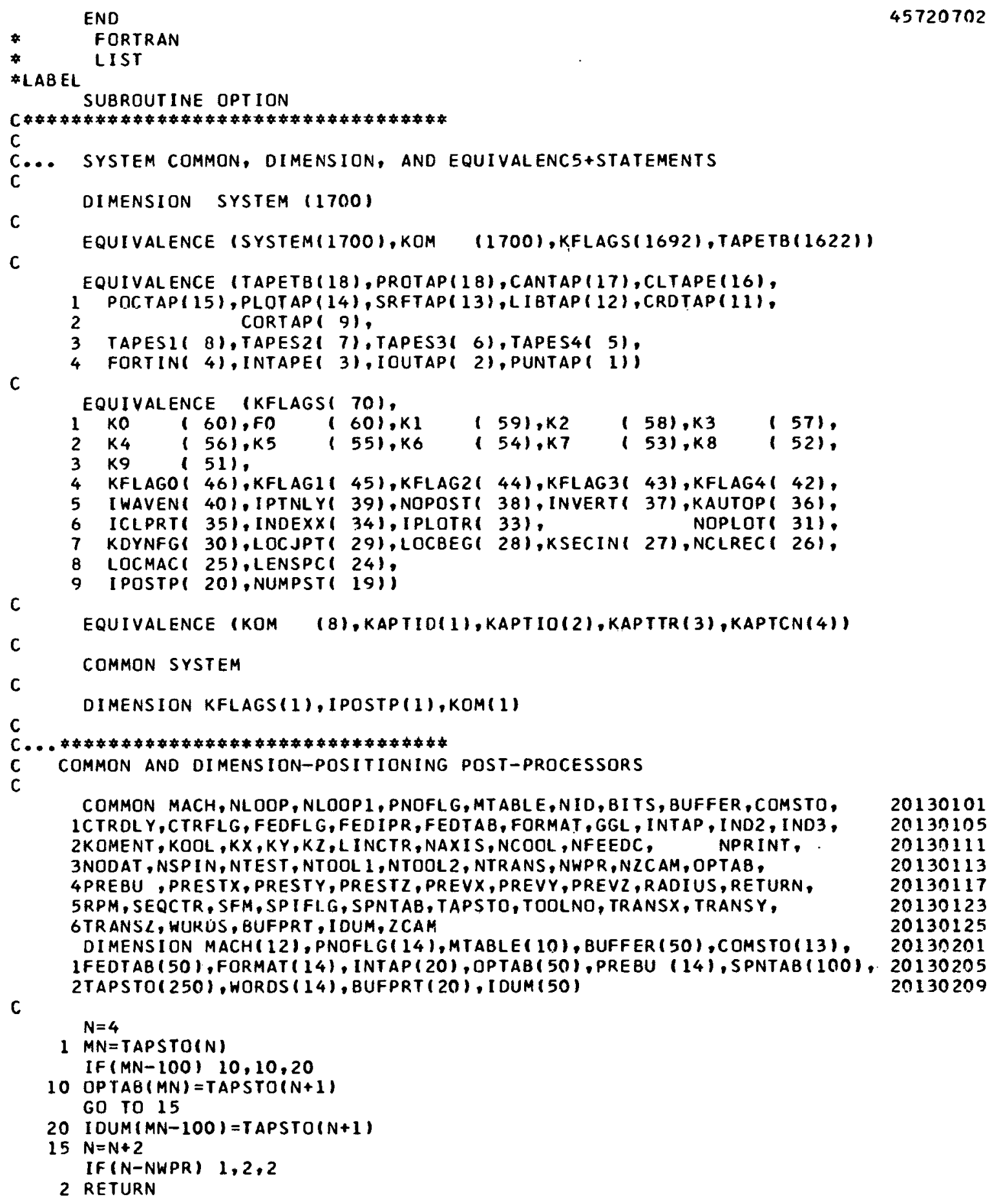




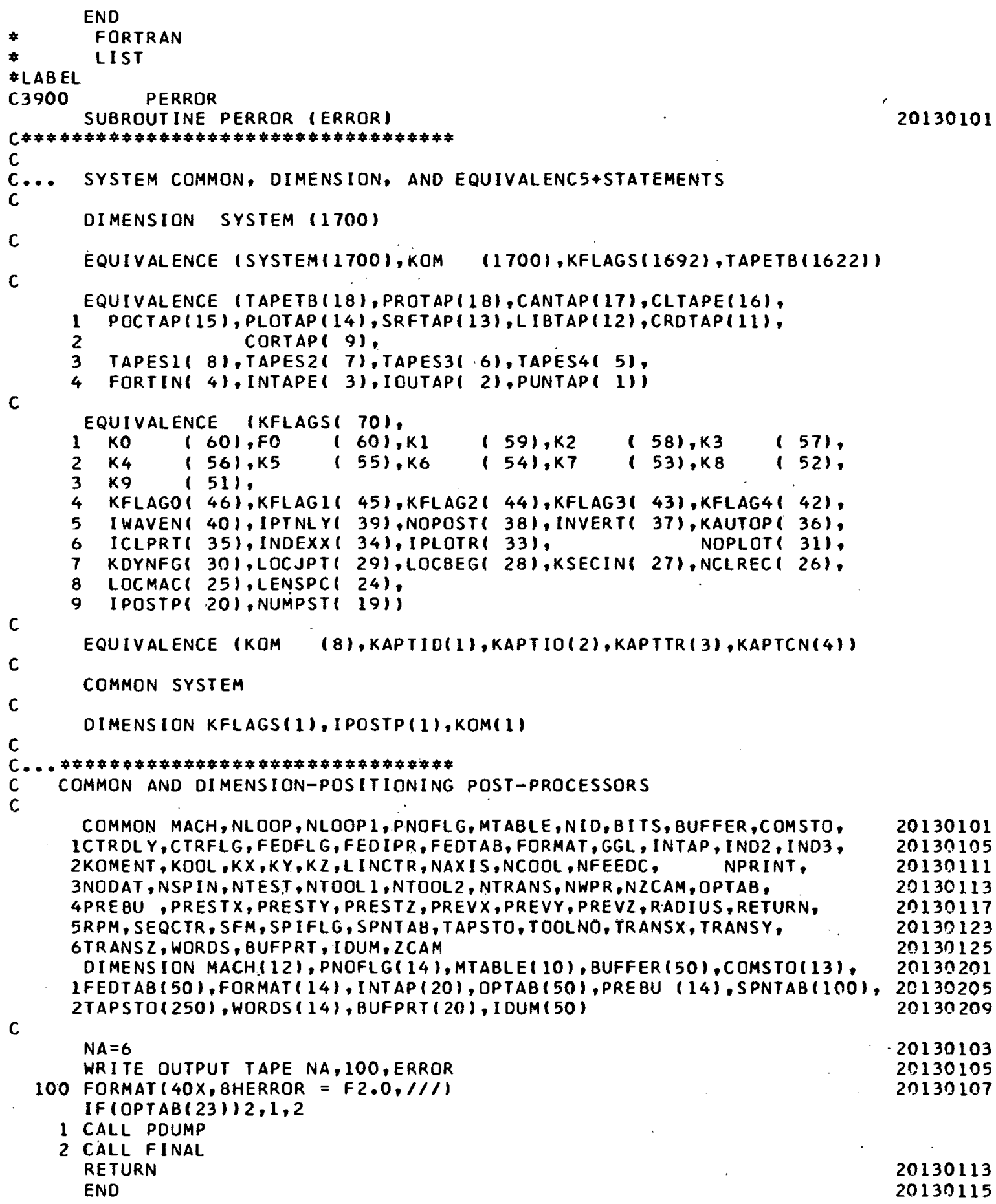


$\begin{array}{ll}* & \text { FORTRAN } \\ \text { * } & \text { LIST } \\ \text { *LABEL } & \\ \text { C4000 } & \text { POSITN }\end{array}$

SUBROUTINE POSITN

20130101

C

C

C... SYSTEM COMMON, DIMENSION, AND EQUIVALENC5+STATEMENTS

DIMENSION SYSTEM $(1700)$

C

EQUI VALENCE (SYSTEM(1700), KOM (1700),KFLAGS(1692),TAPETB(1622))

EQUIVALENCE (TAPETB(18), PROTAP $(18)$, CANTAP $(17), C L T A P E(16)$,

1 POCTAP (15), PLOTAP (14), SRFTAP (13), L IBTAP ( 12), CRDTAP (11).

2 CORTAP 9 ),

3 TAPES1 1 8),TAPES2 ( 7),TAPES3( 6),TAPES4( 5$)$,

C

FORTINI 4), INTAPE( 3), IOUTAP( 2 ), PUNTAP ( 11 )

EQUIVALENCE (KFLAGS(70),

$1 K 0 \quad(60), F 0 \quad(60), K 1 \quad(59), K 2 \quad(58), K 3)(57)$,

$2 K 4(561, K 5 \quad(55), K 6 \quad(54), K 7 \quad(53), K 8 \quad(52)$,

3 K9 (51),

4 KFLAGO ( 46), KFLAG1 ( 45), KFLAG2 ( 44), KFLAG3 ( 43), KFLAG4 ( 42),

5. I WAVEN ( 40), IPTNLY( 39), NOPOST (38), INVERT( 37 ), KAUTOP ( 36$)$,

6 ICLPRT( 35), INDEXX( 34), IPLOTR( 33), NOPLOT( 31$)$,

7 KOYNFG( 30$)$, LOCJPTI 29), LOCBEG( 28 ), KSECIN( 271 , NCLREC 26$)$,

8 LOCMACI 25), LENSPCI 241 ,

C

IPOSTP( 201 , NUMPSTI 19)

EQUIVALENCE (KOM (8), KAPTID(1), KAPTIO(2), KAPTTR(3), KAPTCN(4))

C

COMMON SYSTEM

$\mathrm{C}$

DIMENSION KFLAGS(1), IPOSTP(1),KOM(1)

$\mathrm{C}$

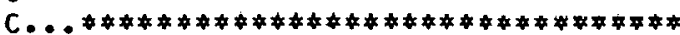

C COMMON AND DIMENSION-POSITIONING POST-PROCESSORS

C

C

C

COMMON MACH, NLOOP, NLOOP 1 , PNOFLG, MTABLE, NID, BITS, BUFFER, COMSTO, 1CTRDLY, CTRFLG, FEDFLG, FED IPR, FEOTAB, FORMAT, GGL, INTAP, IND2, IND3, 2KOMENT, KOOL, KX,KY,KZ, LINCTR, NAXIS, NCOOL, NFEEOC, NPRINT. 3NODAT, NSP I N, NTEST, NTOOL 1 , NTOOL 2 , NTRANS, NWPR, NZCAM, OPTAB, 4PREBU, PRESTX, PRESTY, PRESTZ, PREVX, PREVY, PREVZ, RADIUS, RETURN, SRPM, SEQCTR, SFM, SPIFLG, SPNTAB, TAPSTO, TOOLNO, TRANSX, TRANSY,

- OTRANSZ, HORDS, DUFPRT, IDUM, ZCAM

DIMENSION MACH (12), PNOFLG( 14$)$, MTABLE( 10), BUFFER (50),COMSTO (13), IFEOTAB (50), FORMAT $(14)$, INTAP $(20)$, OPTAB $(50)$, PREBU $(14)$, SPNTAB $(100)$, 2TAPSTO $(250)$, WOROS $(14)$, BUFPRT $(20)$, I OUM (50)

MAIN CONTROL OF POSITIONING POSTPROCESSORS

20130101 20130105 20130111

20130113 20130117 20130123 20130125 20130201

20130205 20130209

20130102 20130103 20130105

$\operatorname{KFLAGS}(50)=0$

CALL PREINT(O)

CALL INIT

$K L=M T A B L E(6)$

1 CALL READER IF (RETURN) $3,6,4$
20130107

20130109 


$$
\begin{aligned}
& \text { C } \\
& \text { C } \\
& \text { C } \\
& \\
& \text { C } \\
& \text { C } \\
& \text { C } \\
& \\
& \text { C } \\
& \text { C } \\
& \text { C }
\end{aligned}
$$

ERROR RETURN

$3 E R R O R=1.0$

2 CALL PERROR (ERROR)

20130113

20130114

20130115

20130117

C

END OF FILE RETURN

4 CALL TAPEOP (CLTAPE, 3, RETURN)

20130121

20130122

20130124

20130201

20130203

20130205

20130206

20130207

$6 N=I D U M(29)$ GO TO $(53,51,55,51,51), N$

53 SEQCTR = FLOATF(INTAP (1))-OP TAB(22) GO TO 51

55 SEQCTR = INTAP $(1)$

51 IND2 = INTAP ( 2)/1000 IFI IND2-16I5,5,1

5 GO TO $(1,10,1,1,30,1,1,1,1,1,1,1,1,4,1,1)$, INO2

$\mathbf{C}$
$\mathbf{C}$
$\mathbf{C}$

AUXILIARY FUNCTIONS

10 IF(INTAP(3)-1)1,11;7

11 CALL ENO

CALL FINAL

CALL PDUMP

CALL DESPAT

7 IF (INTAP $(3)-3) 8,38,9$

8 CALL STOP

GO TO 1

38 CALL OPSTOP GO TO 1

9 IF (INTAP (3)-5) $1,12,15$

12 CALL RAPID GO TO 1

15 IF (INTAP (3)-1000) 1,1,16

16 IND3=INTAP(3)-1000 IF(IND3-61) 13,1,1

1360 To $11,1,1,1,1,25,1,1,18,39$,

$1 \quad 1,1,36,1,1,1,1,1,23,1$.

$2 \quad 19,26,1,1,27,28,1,1,1,20$,

$3 \quad 21,1,1,1,1,1,22,1,1,1$.

$4 \quad 1,1,1,24,1,29,1,33,1,1$,

$5 \quad 34,1,1,1,37,1,46,1,1,1,1,1 N D 3$

18 CALL FEDRAT

GO TO 1

19 CALL DISPLY GO TO 1

20. CALL COOLNT GO TO 1

21 CALL SPINDL GO TO 1

22 CALL TRANS GO TO 1

23 CALL SEQNO(1) GO TO 1

24 CALL MESSAG

20130211

20130213

20130215

20130216

20130217

20130221

20130223

20130225

45720817

45720818

45720819

20130301

20130303

45720820

20130313

20130315

20130317

20130319

20130321

20130323

20130401

20130403

20130405

20130407

45720813 
GO TO 1

37 CALL LOADTL

GO TO 1

39 CALL DELAY

GO TO 1

25 CALL REWIND

26 CALL AUXFUN GO TO 1

27 CALL TOOLN GO TO 1

28 CALL ROTABL GO TO 1

29 CALL INSERT GO TO 1

33 CALL PREFUN GO TO 1

34 CALL OPIION GO TO 1

$36 \mathrm{CALL}$ LEADER GU TO 1

46 CALL. C.I.RSRF GO TO 1

30 IF(INTAP (3)-2) $1,1,31$

31 IF (INTAP (3)-4) 32,50,32

20130501

50 INTAP(3) $=5$

TAPSTO $(6)=$ TAPSTO $(6)+P R E V X$ TAPSTO $(7)=$ TAPSTO $(7)+$ PREVY TAPSTO $(8)=$ TAPSTO $(8)+P R E V Z$

$32 k X=6$

NTEST $=5$

$K Y=7$

$K Z=8$

35 CALL DATA

IF (NTEST) $1,41,41$

41 I $F(K L-13) 42,43,42$

43 DO $44 \quad I=3,5$

44 PREBU ( I ) =B I TS GO TO 45

42 CALL CARD CALL PRINT

45 NTEST $=$ NTEST +3 IF (NTEST-NWPR I 40,1,1

$40 k X=K X+3$

$K Y=K Y+3$

$K Z=K Z+3$

GO TO 35

END

20130505

20130506

20130507

20130509

20130511

45721101

45721102

45721.103

45721104

45721105

20130515

45721106

20130519

20130521

20130523

20130524

20130525

20130526

* Fortran

* List

* $\angle A B E L$

C4200 PREFUN

SUBROUTINE PREFUN

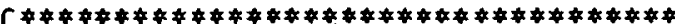

C

C... SYSTEM COMMON, DIMENSION, AND EQUIVALENC5+STATEMENTS

C

C

DIMENSION SYSTEM 117001 


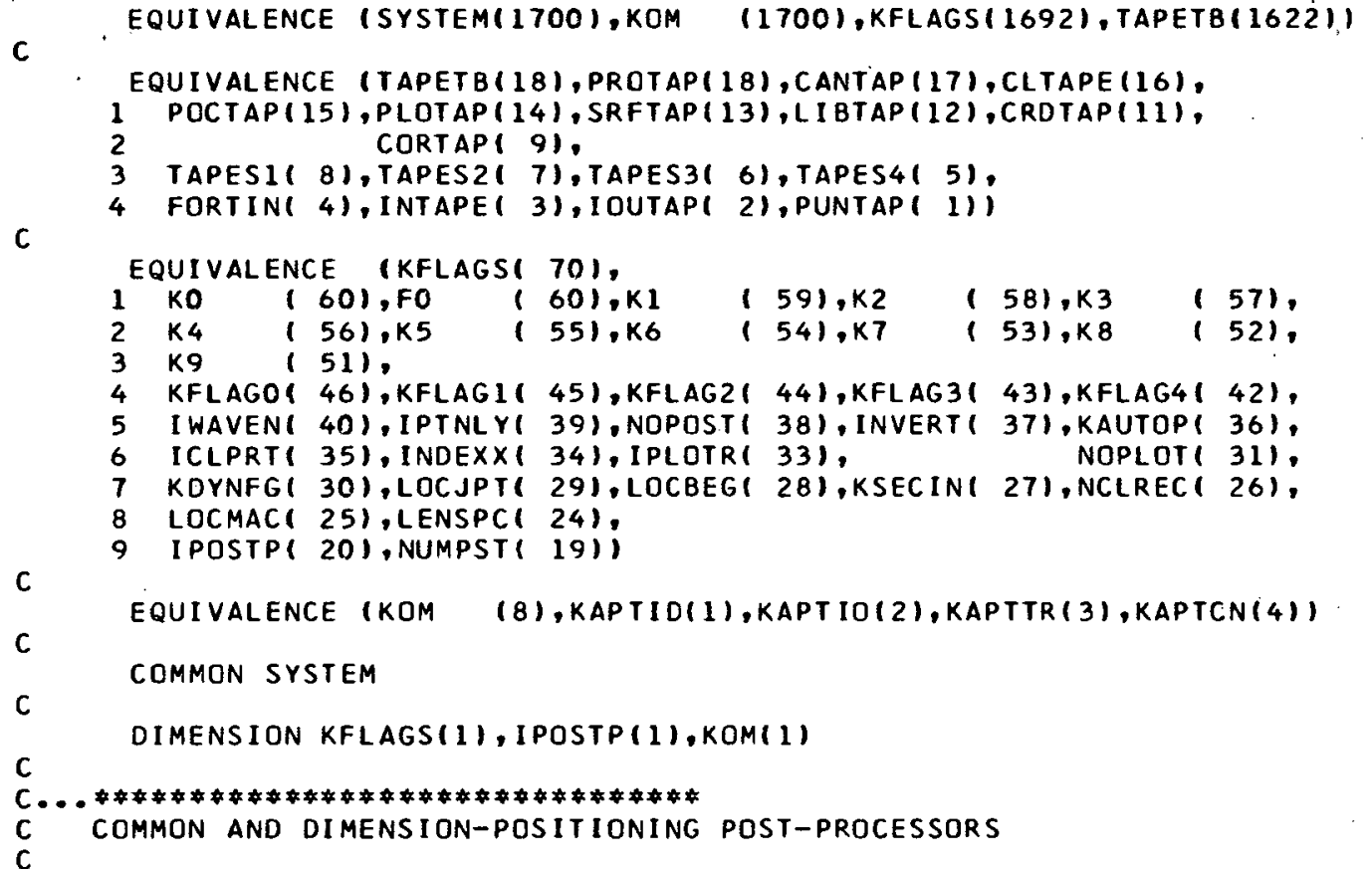

COMMON MACH,NLOOP,NLOOPI, PNOFLG, MTABLE,NID,BITS,BUFFER,COMSTO, 20130101 1CTRDLY,CTRFLG, FEDFL G, FEDIPR, FEOTAB, FORMAT, GGL, INTAP, IND2, IND3, 20130105 2KOMENT, KOOL, KX, KY, KZ, LINCTR, NAXIS, NCOOL, NFEEDC, NPRINT, 3NODAT, NSPIN, NTEST, NTOOL 1, NTOOL2, NTRANS, NHPR, NZCAM, OPTAB, 4PREBU, PRESTX, PRESTY, PRESTZ, PREVX, PREVY, PREVZ, RADIUS, RETURN, SRPM, SEQCTR, SFM, SP I FLG, SPNTAB, TAPSTO, TOOLNO, TRANSX, TRANSY, GTRANSZ, WORDS, BUFPRT, IOUM, ZCAM DIMENSION MACH (12), PNOFLG ( 14), MTABLE( 10), BUFFER(50),COMSTO (13), 1FEDTAB (50), FORMAT (14), INTAP(20), OP IAB (50), PREBU (14), SPNTAB (100), 20130205 2TAPSTO $(250)$, WOROS $(14)$, BUFPRT $(20)$, IDUM $(50)$ $201301-11$ 20130113 $2 n 130117$ 20130123 $2 n 130125$ 20130201 20130209

C.

$N=M T A B L E(6)$

IF $(N-15) 2,2,1$

2 GO TO $(1,1,1,1,1,1,1,3,3,3,1,1,1,3,1), \mathrm{N}$

3 IF (NWPR -4$) 1,4,4$

4 IF(IDUM(16)-1)11,10,11

10 IFLAG $=1$ PRE SAV $=O P T A B(5)$

11 I DUM (16) =1 OPTAB (5) = TAPSTO(4) IF $(N-8) 1,5,1$

5 IF (IFLAG-1)13,12,13

12 OPTAB (5)=PRESAV GO TO 7

$13 L=O P T A B(5)+.1$ I $F(L-61) 6,1,6$

6 IF (L-80) 7, I, 7

7 SEQCTR $=S E Q C T R+O P T A B(22)$ PREBU(1) $=606000000000$ $\operatorname{IDUM}(16)=0$ PREBU $(2)=O P T A B(5)$ 


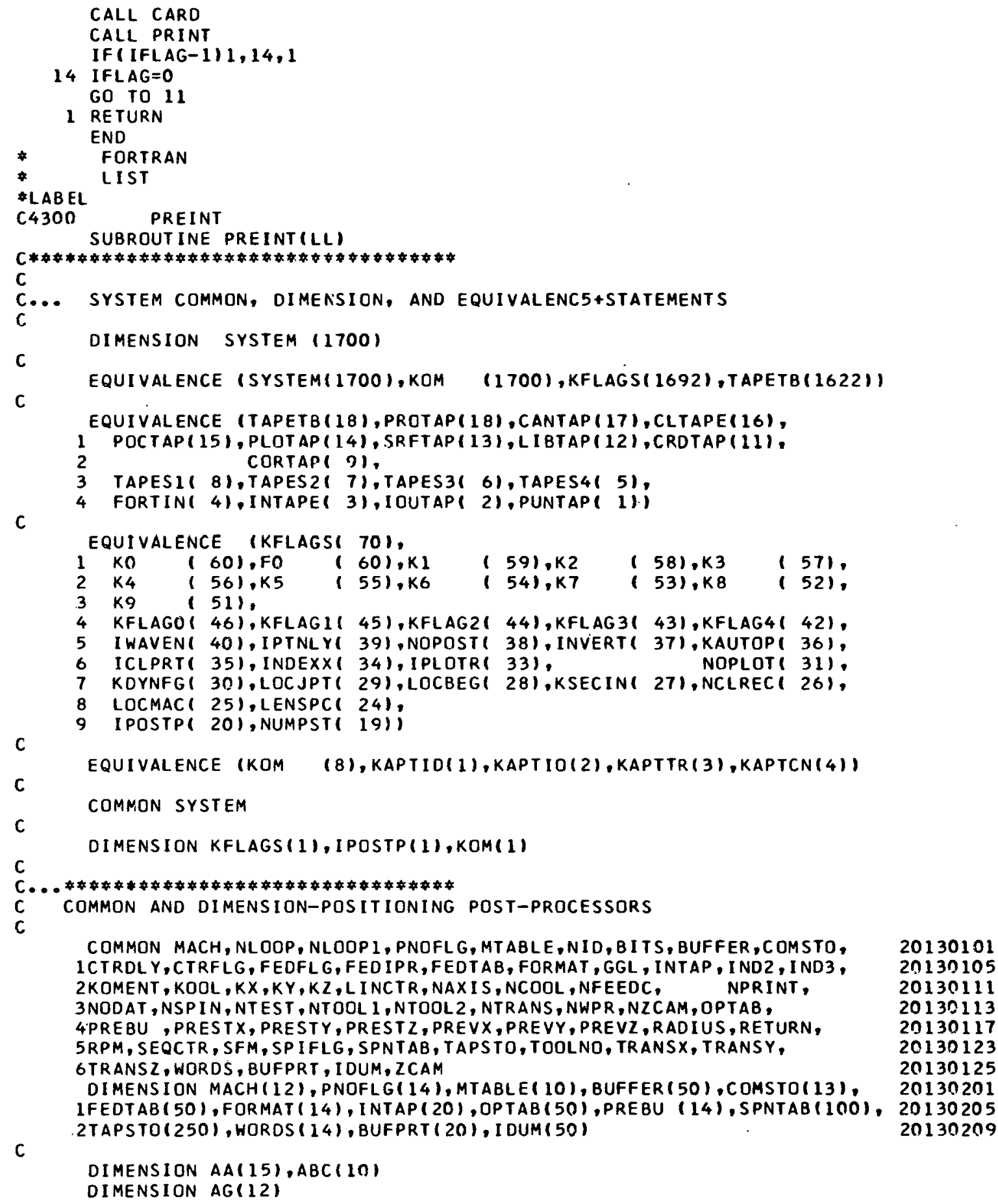


$N N=M T A B L E(6)$

IFILL) $301,300,301$

B $300 A E=606060606060$

DO $799 \quad J 7=1,12$

$799 A G(J 7)=A E$

$A G(9)=606044000053$

$A G(10)=606044000053$ $A G(11)=440000757575$ $N=-72$

$\mathrm{J}=0$

$K=1$

CALL PUNCHA $(N, A G, J, K)$

$N=-6$

CALL PUNCHA $(N, A G(10), J, K)$

$N=-12$

CALL PUNCHA(N,AG(11),J,K)

301 IF (NN-15) 15,15,35

15 GO TO $(1,2,3,4,5,6,7,8,9,10,11,12,13,14,35), \mathrm{NN}$

$1 A A(1)=273124436662$

$A A(2)=736063212243$

$A A(3)=257360016060$

$A A(4)=606060606060$

GO TO 25

$2 A A(1)=246543312527$

$A A(2)=736041312744$

$A A(3)=314373600160$

$A A(4)=606060606060$ GO TO 25

47490215

3 AA $(1)=264662243142$ $A A(2)=736041312722$ $A A(3)=.465173600160$ $A A(4)=606060606060$ GO TO 25 .

20130121

$4 A A(1)=302521432473$ $A A(2)=602451314343$ $A A(3)=736001606060$ $A A(4)=606060606060$ GO TO 25

$5 A A(1)=432151305173$ $A A(2)=602451314343$ $A A(3)=736001606060$ $A A(4)=606060606060$ GO TO 25

6 AA $(1)=214425512345$ $A A(2)=736063212243$ $A A(3)=257360016060$ $A A(4)=606060606060$ GO TO 25

$7 A A(1)=424531273063$ $A A(2)=736041312722$ $A A(3)=465173600160$ $A A(4)=606060606060$ GO TO 25

8 AA $(1)=602264512773$ $A A^{\prime}(2)=2451.31434373$ $A A(3)=600160606060$ $A A(4)=606060606060$ GO TO 25

9 AA $(1)=273124436662$ 
$A A(2)=736024513143$

$A A(3)=437360016060$

$A A(4)=606060606060$ GO TO 25

$10 A A(1)=436423216273$

$A A(2)=602246514431$

$A A(3)=437360026060$

$A A(4)=606060606060$ GO TO 25

11 AA $(1)=273124436662$ $A A(2)=736024513143$ $A A(3)=437360026060$ $A A(4)=606060606060$ GO TO 25

$12 A A(1)=475121636366$ $\triangle A(2)=736024513143$ $A A(3)=437360016060$ $A A(4)=606060606060$ GO TO 25

$13 A A(1)=425145635173$ $A A(2)=604423302345$ $A A(3)=637360622551$ $A A(4)=316225606060$ GO TO 25

$14 A A(1)=273124436662$ $A A(2)=736022465144$ $A A(3)=314373600631$ $A A(4)=452330606060$ GO TO 25

$25 K=14$

DO $30 \quad J=5,15$

$K=K-1$

45720101 45720102 45720103 45720104

47490216 47490217 47490218 47490219 47490220 $2013 \cap 201$

30.AA $(J)=P N O F L G(K)$

20130205 PNOFLG (14) = AA(1) GOTO $(26,26,26,26,27,26,26,27,27,26,27,27,27,27,27), N N$ 20130207

$26 \mathrm{~N}=+90$

CALL SWAP(AA)

CALL PARNEW(N, AA(15)) GO TO 331

$27 N=-90$

CALL PARNOM $(N, A A)$

20130211

$331 C=B$ ITS

CALL PUNIDN(C)

$B=7.0$

CALL PUNSEQ(B)

IFILL) $35,31,35$

$31 . M=-60$

$0033 L=1,9$

B $33 A B C(L)=131313131313$

GO TO $(36,36,36,36,36,36,36,39,36,36,36,36,36,36,36), \mathrm{NN}$

$\forall \quad 39 A B C(10)=131313133453$

GO TO 37

$8 \quad 36 \quad A B C(10)=131313131353$

$37 \mathrm{~J}=0$

$K=1$

34 CALL PUNCHAIM, ABC, J,K)

35 RETURN

20130213

END

FORTRAN 


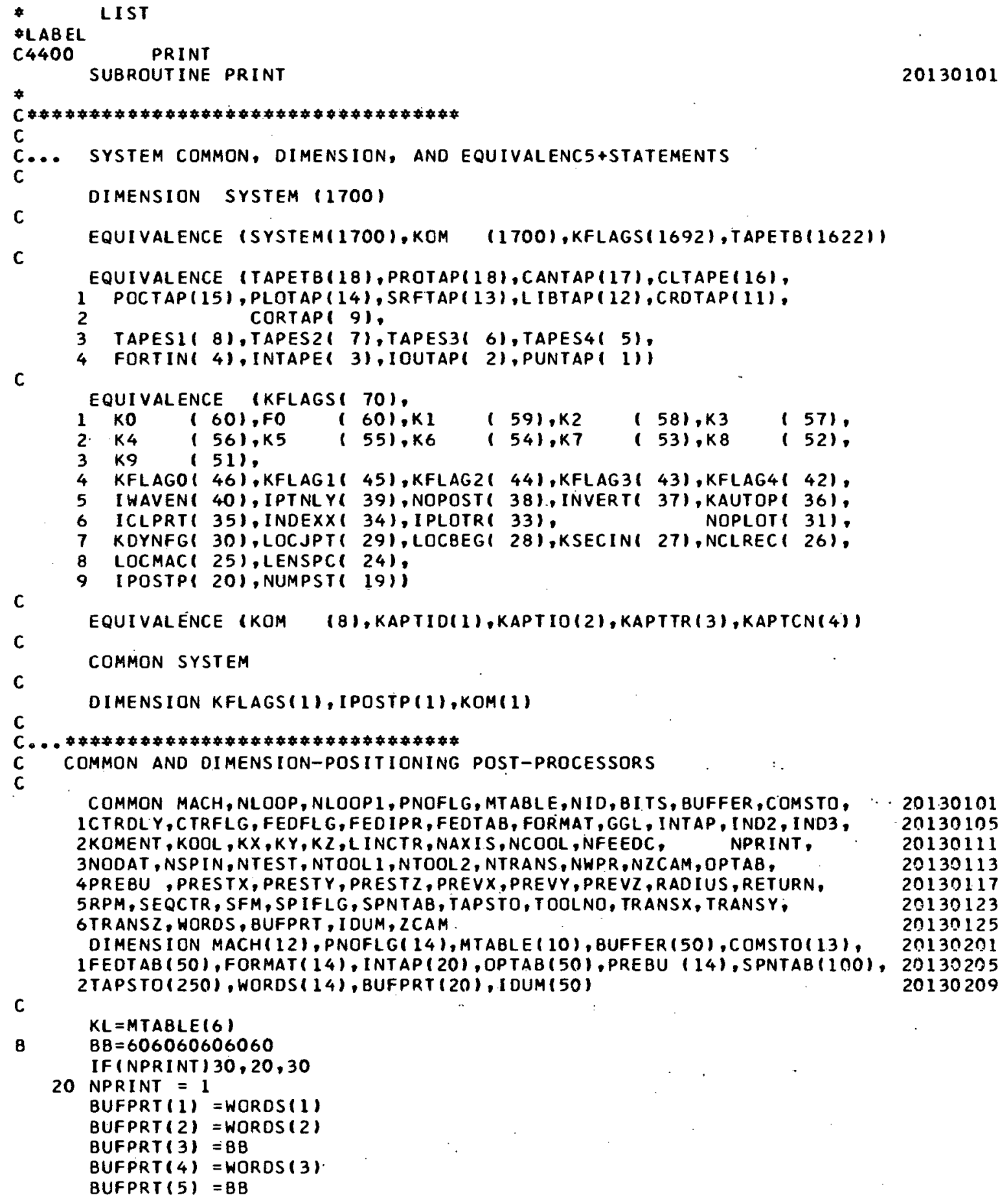




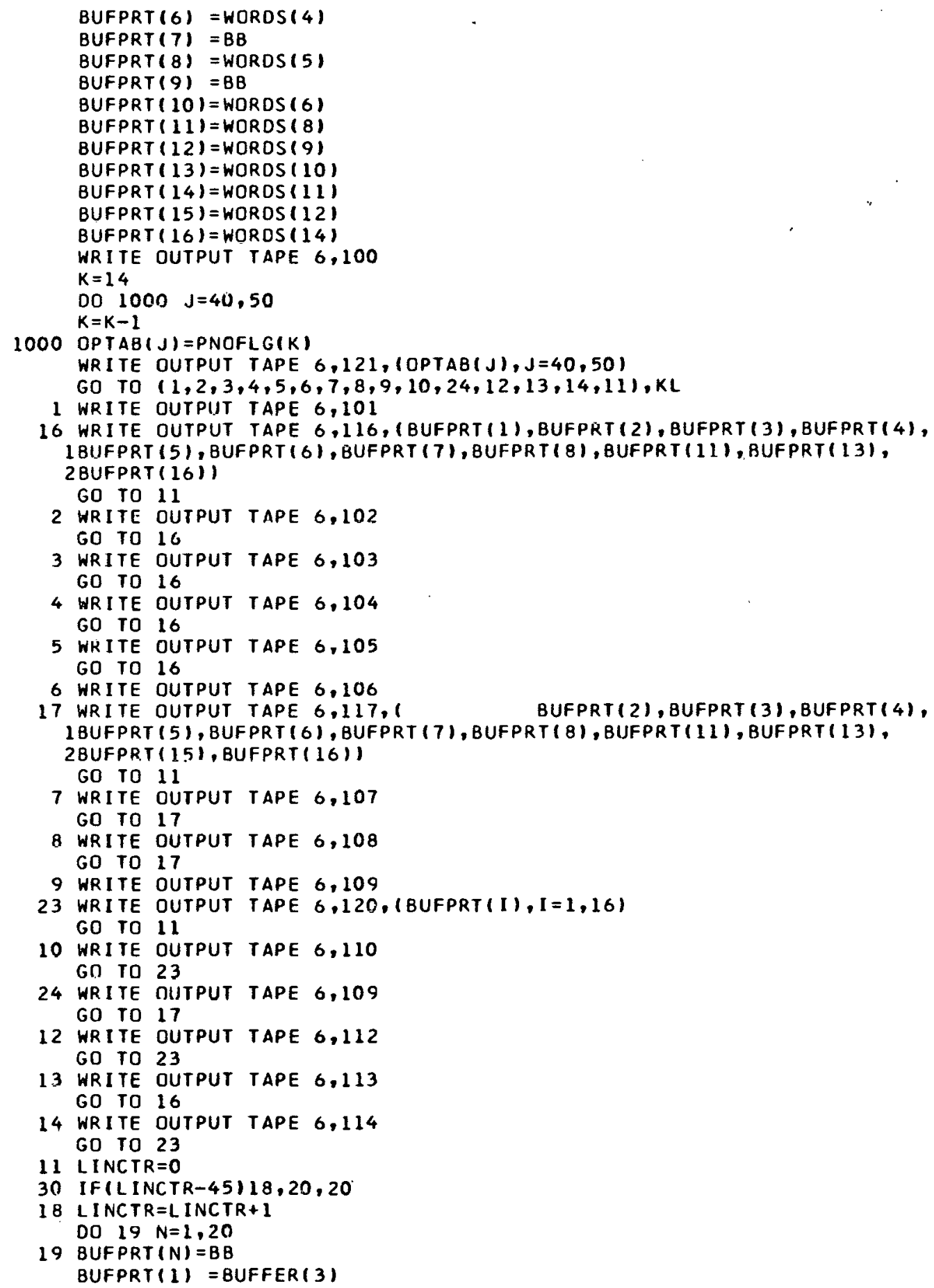


BUFPRT (2) = BUFFER $(6)$

BUFPRT $(11)=B U F F E R(27)$

BUFPRT $(12)=$ BUFFER $(30)$

BUFPRT (13)= BUFFER (33)

BUFPRT $(14)=$ BUFFER $(36)$

BUFPRT $(15)=$ BUFFER $(39)$

BUFPRT $(16)=$ BUFFER $(45)$

N3 $=3$

DO $21 \quad I=3,6$

IF(PREBU(I)-BITS) 22,21,22

$22 A A=F O R M A T(I) / 10.0$

$\mathrm{N} 2=\mathrm{AA}+.001$

$B=N 2$

$E=A B S F(F O R M A T(I)-(B * 10.01)+.001$

$N \mathbf{l}=-E$

CALL CONBCO (PREBU(I), BUFPRT(N3),N2,N1)

$21 N 3=N 3+2$

GO TO $(41,41,41,41,41,42,42,42,43,43,42,43,41,43,50), \mathrm{KL}$

47490305

41 WRITE OUTPUT TAPE 6,118 , (BUFPRT (1), BUFPRT $(2)$, BUFPRT $(3), B U F P R T(4)$, 1 BUFPRT ( 5 ), BUFPRT (6), BUFPRT (7), BUFPRT ( 8), BUFPRT 111, BUFPRT (13), 2BUFPRT (.16))

GO TO 50

43 WRITE DUTPUT TAPE $6,118,($ BUFPRT $(I), I=1,16)$

GO TO 50

42 I BOB $=S E Q C T R$

WRITE OUTPUT TAPE $6,119,1$ (BOB,BUFPRT (2), BUFPRT(3), BUFPRT(4), 1BUFPRT (5), BUFPRT (6), BUFPRT (7), BUFPRT ( 81, BUFPRT 1111 , BUFPRT (13), 2BUFPRT (15), BUFPRT $(16))$

50 DO $51 \quad I=1,14$

51 PREBU $(I)=B I T S$

RETURN

100 FORMAT (IHI)

101 FORMAT (26H G. + L. POSITIONING TABLE///)

102 FORMAT (ITH DEVLIEG JIG MILL/I/)

103 FORMAT (28H FOSDICK POSITIONING MACHINE///)

104 FORMAT ( $18 \mathrm{H}$ HEALD - HEALDRILL///)

105 FORMAT (13H LAHR - DRILL///)

106 FORMAT (17H AMERICAN - DRILL///)

107 FORMAT (17H KNIGHT JIG BORER///)

108 FORMAT (13H BURG - DRILL///I

109 FORMAT (14H G. + L. DRILL///)

110 FORMAT (18H LUCAS BORING MILL///)

112 FORMAT 20 H PRATT-HHITNEY DRILL///

113 FORMAT ( 13 H KRNTR-MCHCNT///)

114 FORMAT (15H G. + L. BORMIL///)

45720110

116 FORMAT $(11 A 6 / / / 1$

117 FORMATI6H N,11A6///I

118 FORMAT(16A6)

119 FORMAT $(1 X, 15,1146)$

120 FORMAT ( $16 A 6 / / /)$

121 FORMAT (IH, 11 A6,53X)

END

* Fortran

SUBROUTINE P.RINTA

c

C... SYSTEM COMMON, DIMENSION, AND EQUIVALENC5+STATEMENTS

60009300 60009400 60009500 DIMENSION SYSTEM (1700) 60009600 
C

C

C

C

C

C

c

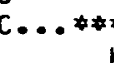

1

c
EQUIVALENCE (SYSTEM(1700), KOM

EQUIVALENC 1 POCTAP (15), PLOT AP (14), SRFTAP( 13$)$, LI I BTAP (12), CRDTAP (11), CORTAP( 9$)$,

3 TAPESI 18 ), TAPES 21 7), TAPES31 6), TAPES41 51 ,

4 FORTIN( 4$)$, INTAPE 3 ), IOUTAP( 2$)$, PUNTAP ( 111

EQUIVALENCE (KFLAGS( 70$)$,

$\begin{array}{llllllll}1 & K O & (60), F O & (60), K 1 & (59), K 2 & (581, K 3 & (57), \\ 2 & K 4 & (56), K 5 & (55), K 6 & (54), K 7 & (53), K 8 & (52) \text {, }\end{array}$

2 K 150$)$,

KFLAGO ( 46$)$; KF

$(54), K 7$

( 53$), K 8$

(52),

46), KFLAG1( 45), KFLAG2 ( 44), KFLAG31 43), KFLAG4 ( 42), IWAVEN( 40), IPTNLY( 39), NOPOST ( 38), INVERT( 37), KAUTOP( 36), ICLPRT ( 35), INDEXX( 34), IPLOTR( 33), NOPLOT 31 ), KDYNFG( 30$)$, LOCJPT( 29), LOCBEG( 28$),$ KSECINI 271 , NCLREC ( 26), LOCMACI 25), LENSPC( 24),

9 IPOSTPI 201 , NUMPST 19 )i

EQUIVALENCE IKOM

$(8)$, KAPTID $(1)$, KAPTIO (2), KAPTTR $(3), \operatorname{KAPTCN}(4))$

COMMON SYSTEM

DIMENSION KFLAGS(1), IPOSTP(1),KOM(1)

\section{$\operatorname{KFLAGS}(50)=\operatorname{KFLAGS}(50)+1$}

RETURN

ENO

*

* $\angle A B E L$

\$4500 PRTON

ENTRY PRTON

PRTON CLA 1,4

STO N

ARS 18

$X C A$

PXD 0.0

MPY NG

$X C A$

ADD $\quad A D R$

STA DO

SXA B.4

CLA $\quad 2,4$

STO BB

AXT 0.4

DO

CLA $\quad 0.4$

STO PRT +5.4

IXI $\quad+1,4,1$

TXL $\quad 00,4,5$

CALL (SPH),PRT

CALL (FIL)

LFTM

CALL

LDQ

STR

CALL

LFTM

(SPH), ACC

BB

(FIL)
60009700

60009800

60009900

60010000

60010100

$6001020 n$

60010300

60010400

6n01050n

60010600

60010700

60010800

60010900

60011000

60011100

60011200

60011300

60011490

6001150 .

60011600

$6001170 n$

60011800

60011900

60012000

60012100

$600122 n$

60012300 


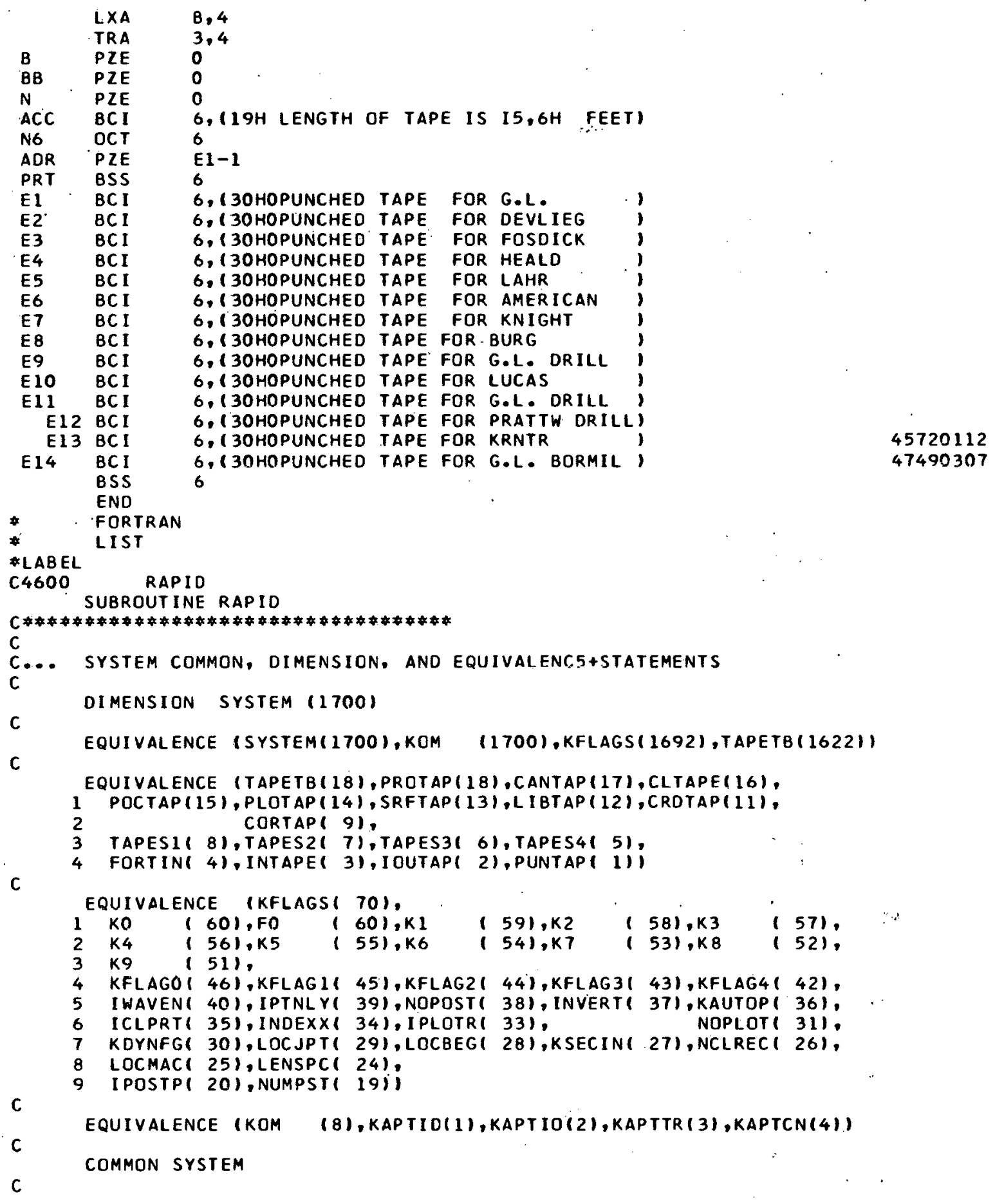




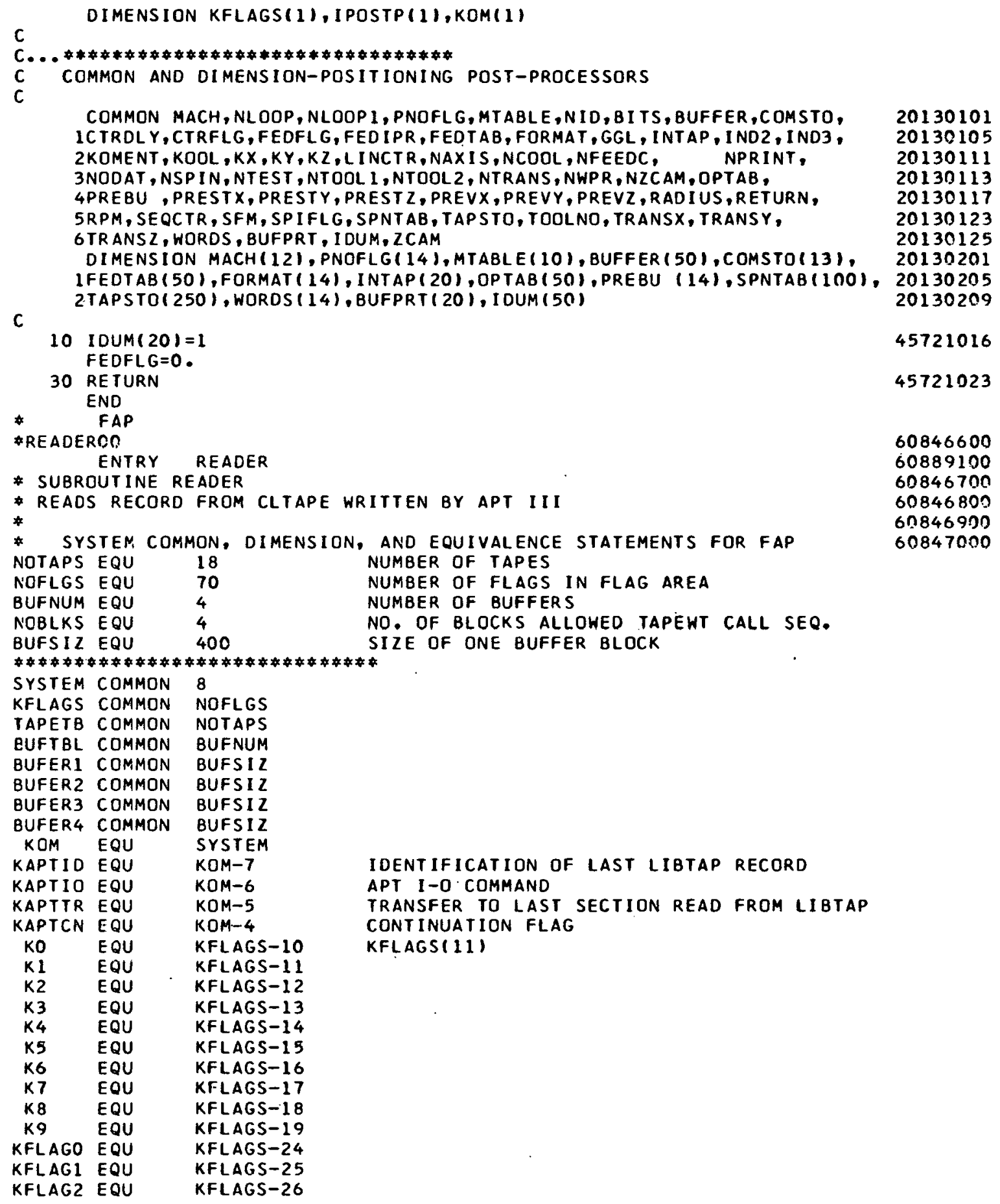




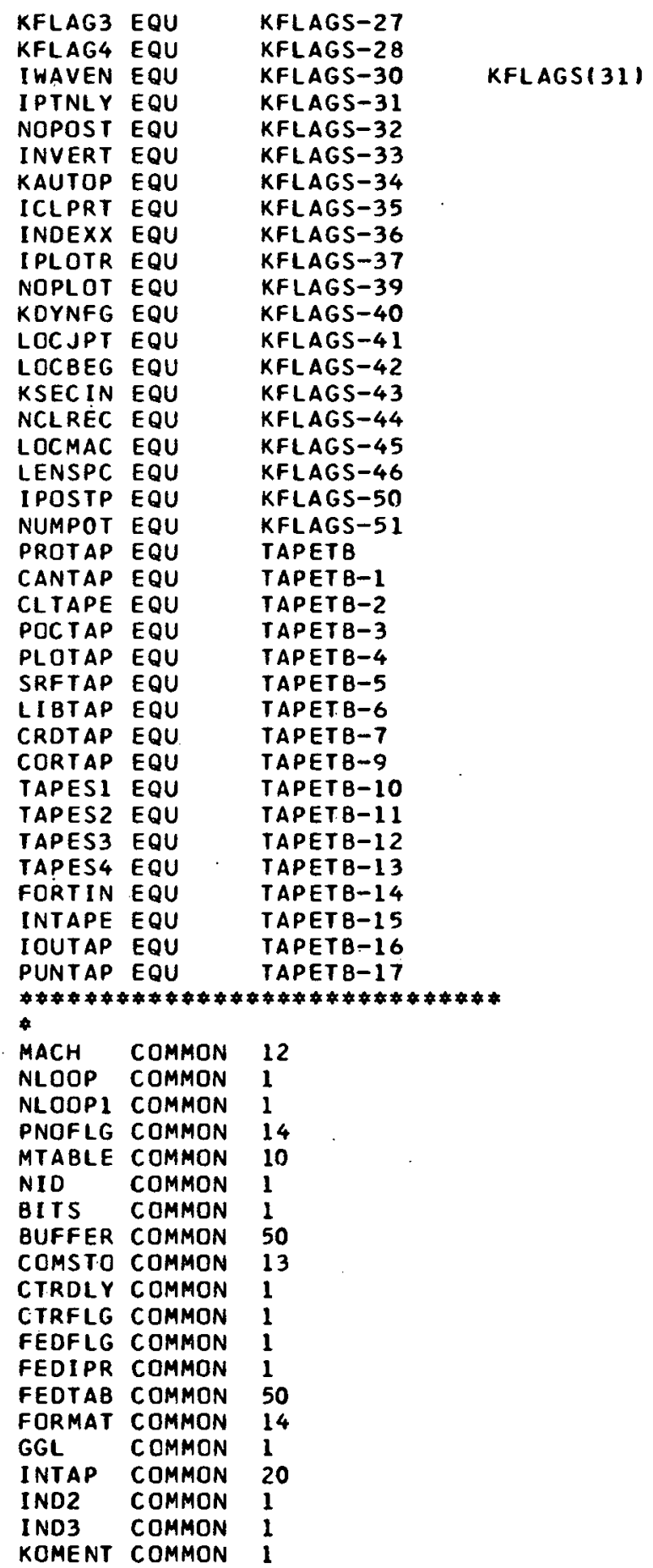




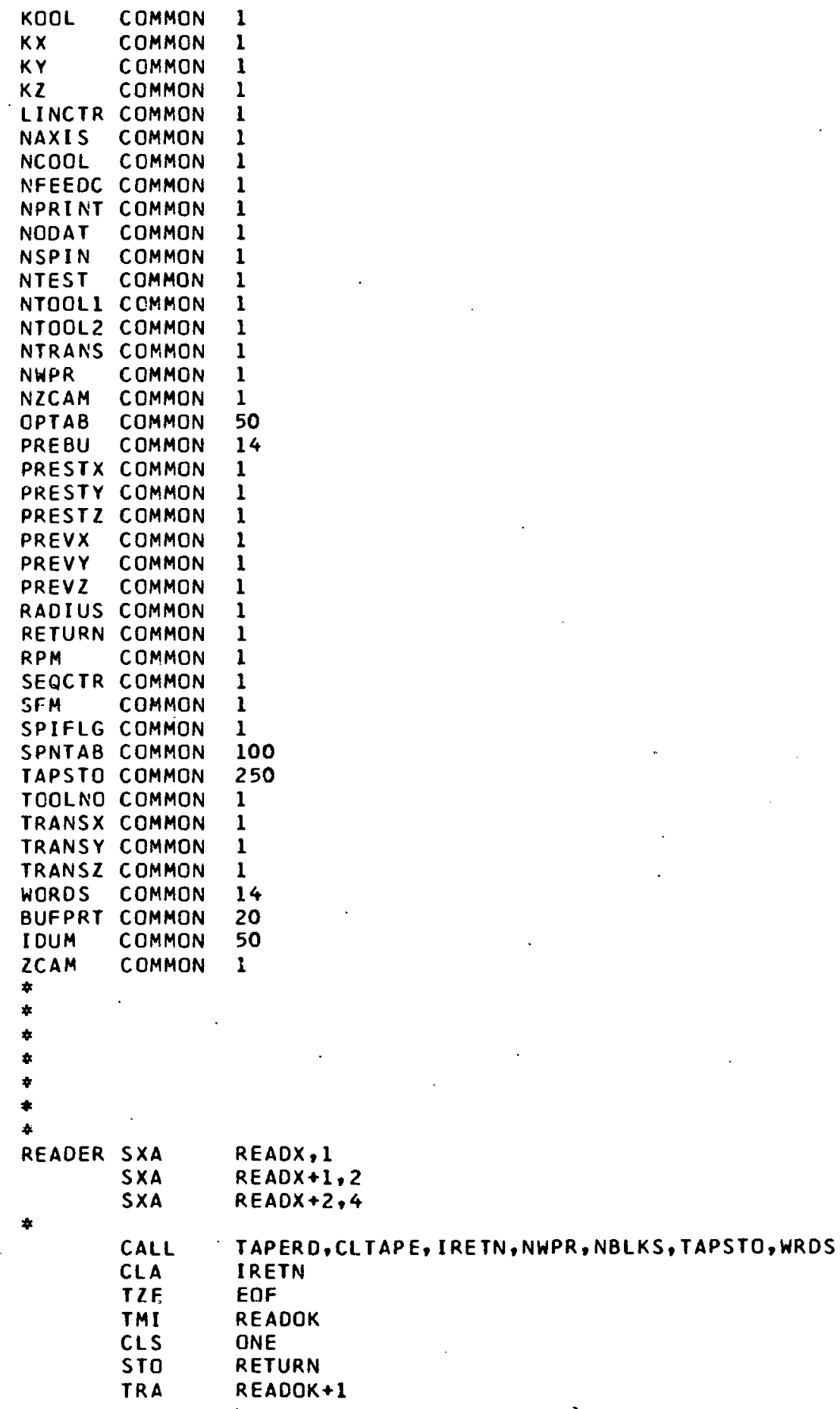

60889000 60889200 60889300 60889400 60889500 60889600 60889700 60889800 60889900 60890000 60890100 60890200 $6089030 n$ 6089040 n 60890500 60890600 


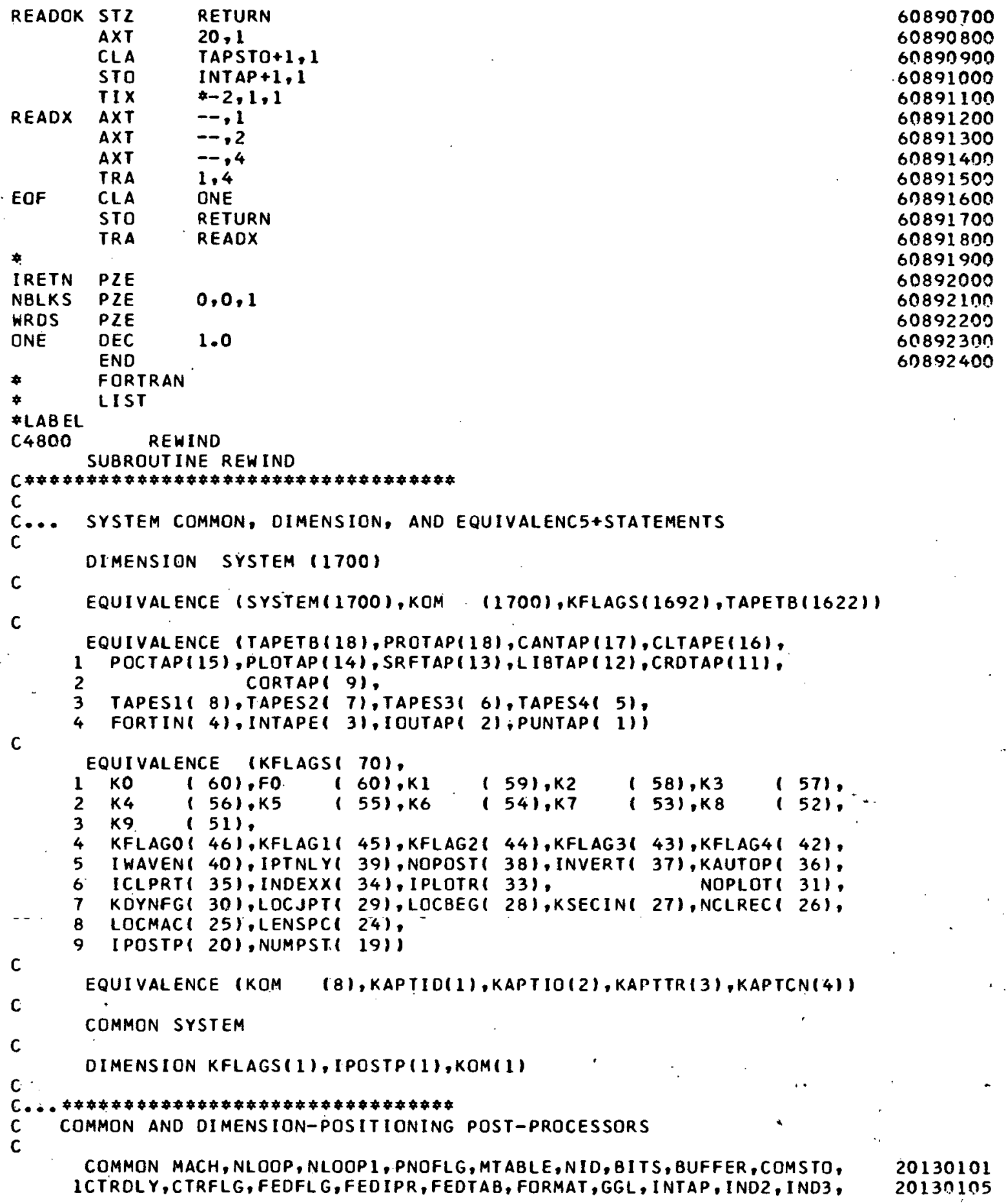


$C$

EQUIVALENCE (KOM (8), KAPTID(1),KAPTIO(2), KAPITR(3), KAPTCN(4))

COMMON SYSTEM

C

DIMENSION KFLAGS(1), IPOSTP(1),KOM(1)

C 


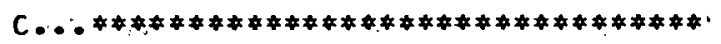

C COMMON AND DIMENSION-POSITIONING POST-PROCESSORS

C

COMMON MACH, NLOOP, NLOOP 1 , PNOFLG, MTABLE, NID, BI TS, BUFFER, COMSTO,

$201 \cdot 30101$

1CTRDL Y, CTRFLG, FEDFLG, FED IPR, FEDTAB, FORMAT, GGL, INTAP, IND2, IND3,

20130105

2KOMENT, KOOL, KX, KY, KZ, LINCTR, NAXIS, NCOOL, NFEEDC, NPRINT,

20130111

3NODAT, NSPIN, NTEST, NTOOL 1 , NTOOL 2 , NTRANS, NWPR, NZCAM, OPTAB,

20130113

4PREBU, PRESTX, PRESTY, PRESTZ, PREVX, PREVY, PREVZ, RAOIUS, RETURN, 20130117

5RPM, SEQCTR, SFM, SPIFLG, SPNTAB, TAPSTO, TOOLNO, TRANSX, TRANSY, $\quad 20130123$

6TRANSZ, WORDS, BUFPRT, IDUM, ZCAM

20130125

DIMENSION MACH (12), PNOFLG(14), MTABLE(10), BUFFER (50), COMSTO(13), IFEDTAB (50), FORMAT $(14)$, INTAP $(20)$, OPTAB $(50)$, PREBU $(14)$, SPNTAB $(100)$,

C 2TAPSTO $(250)$, HORDS $(14)$, BUFPRT $(20)$, IDUM $(50)$

20130201

20130205

20130209

DIMENSION SPEEO(10)

$N=M T A B L E(6)$

IF $(N-10) 1,10,27$

27 If $(N-13) 1,28,1$

10 IF (NWPR -4$) 1,2,3$

2 IF(INTAP. $(4)-1) 6,1,6$

6 OPTAB $(21)=T A P S T O(4)$

GO TO 4

3 IF (NWPR-6) 5, 8,8

5 IF (INTAP $(4)-1) 9,7,9$

7 OPTAB $(21)=$ TAPSTO $(5)$

GO TO 4

9 IFI INTAP (4)-78) 30,11,6

30 IF (INTAP (4)-66)6,29,6

28 IF(NWPR-5) $29,35,29$

29 WRITE OUTPUT TAPE 6,34

34 FORMAT ( 27 H ROTABL STATEMENT INCORRECT) GO TO 1

35 IFIINTAP $(4)-66) 29,33,29$

33 IANGL $=$ TAPSTO(5)+45.

I ANGL $=$ I ANGL $/ 90$

IF (PREVZ-13.9985) 71,70,70

45720917

71 PREVZ $=13.999$

70 PREBU $(1)=S E Q C T R$

PREBU $(5)=$ PREVZ

$S E Q C T R=S E Q C T R+O P T A B(22)$

PREBU $(1)=$ SEQCTR

DO $32 I=1$, I ANGL

PREBU ( 14$)=78$.

CALL CARD

32 CALL PRINT

GO TO 1

$11 \operatorname{OPTAB}(24)=T A P S T O(5)$

GO TO 20

8 IFIINTAP (4)-1)12,13,12

12 I F(INTAP (4)-78):15,11,15

15 OPTAB $(21)=$ TAPSTO $(4)$

IF (INTAP (5)-78) 4,14,4

$4 \operatorname{IDUM}(18)=1$

25. IDUM $(15)=1$

CALL DATA

CALL CARO

CALL PRINT

1 RETURN

14 OPTAB $(24)=$ TAPSTO $(6)$

45720923

45720920

45720921

45720924

45720922

45720925

45721001

45721002

45721003 


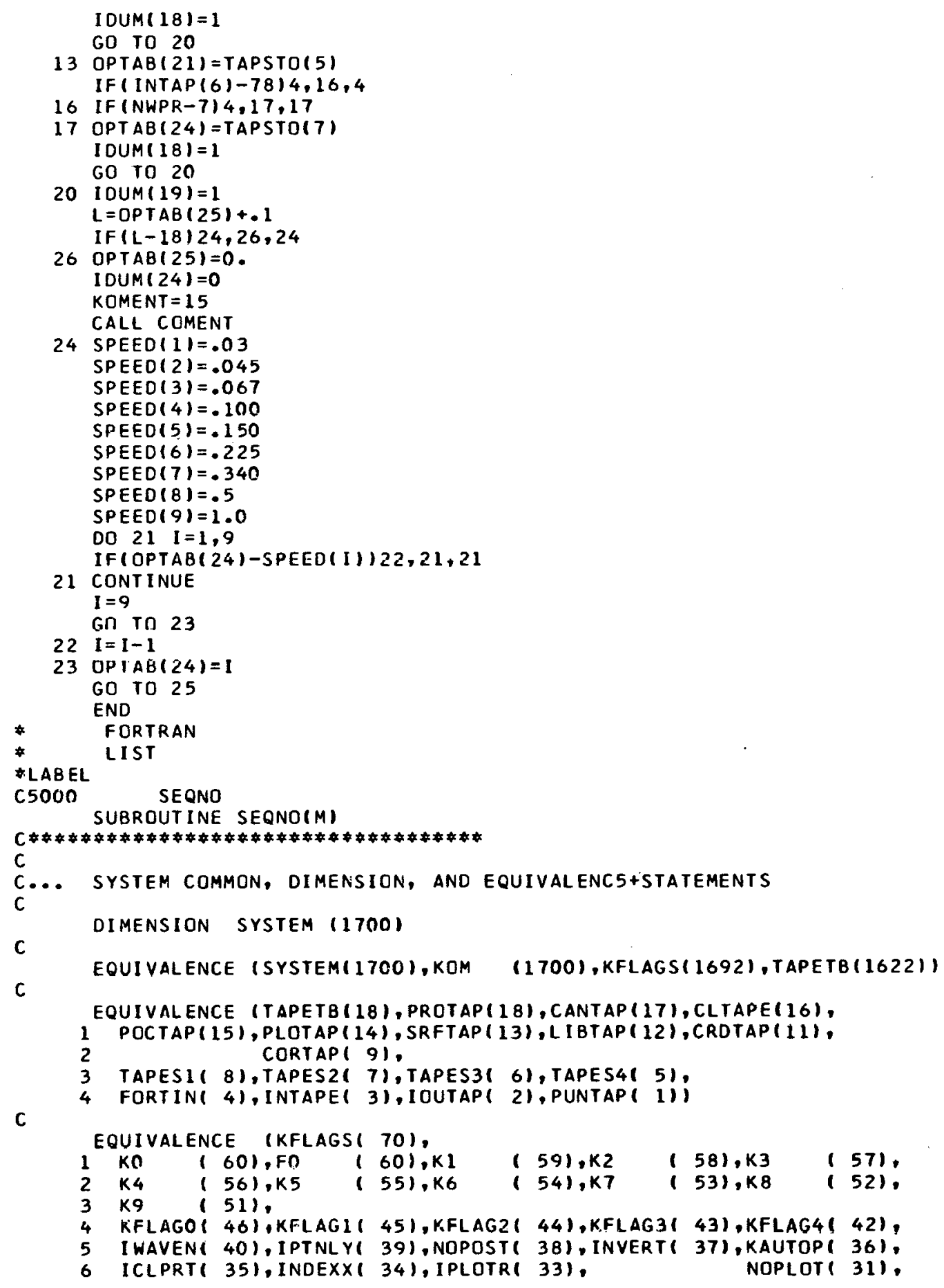

\begin{tabular}{|c|c|c|c|c|c|c|}
\hline & & & & & & \\
\hline & Ko & $601, F 0$ & $601, K 1$ & $591, \mathrm{K2}$ & $581, k 3$ & 571. \\
\hline & K4 & 5 & K6 & 67 & $K 8$ & 521, \\
\hline & K9 & $51 \%$, & & & & \\
\hline & KFLAGO I & FLAGII & FLAG2 I & $\angle A G 31$ & 43), KFLA & 421 \\
\hline & $\begin{array}{l}\text { IWAVENI } \\
\text { ICLPRT }\end{array}$ & $\begin{array}{l}\text { 40), IPTNLYY } \\
35 \text { ), INDEXX ( }\end{array}$ & $\begin{array}{l}\text { 391, NOPOST } \\
34 \% \text {, IPLOTRI }\end{array}$ & $\begin{array}{l}381 \text {, INVERT( } \\
331 \text {. }\end{array}$ & $\begin{array}{l}371 \text {. KAUTOP } \\
\text { NOPLOT }\end{array}$ & $\begin{array}{l}361: \\
311:\end{array}$ \\
\hline
\end{tabular}




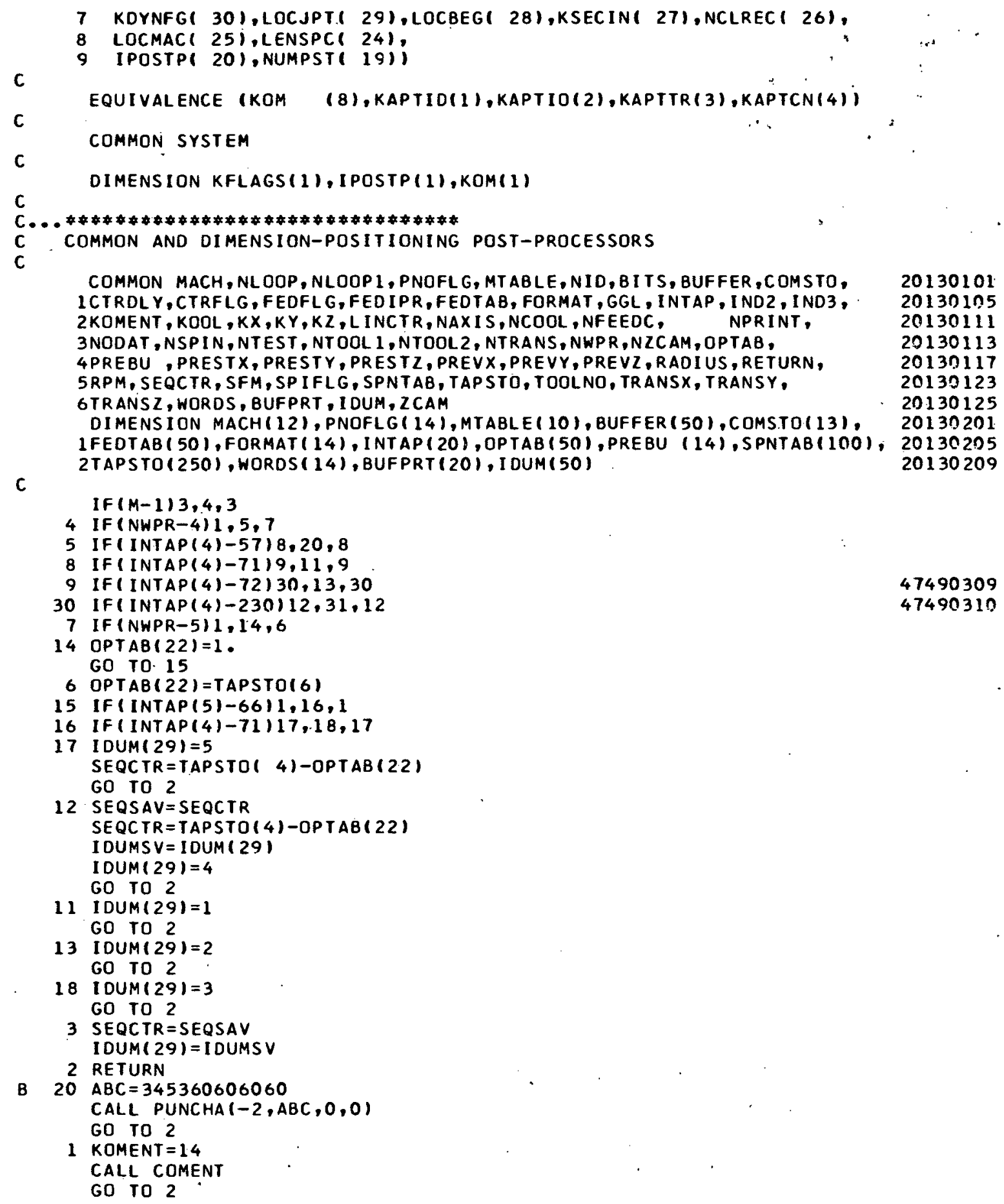


CALL PUNCHAI- $6, A, 0,01$

* fortran

* LABEL

C5100 SETUP

SUBROUTINE SETUP

20130101

C

C

C... SYSTEM COMMON, DIMENSION, AND EQUIVALENC5+STATEMENTS

C

C DIMENSION SYSTEM $(1700)$

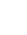

EQUIVALENCE (SYSTEM(1700), KOM (1700),KFLAGS11692), TAPETB(1622))

C

EQUIVALENCE (TAPET $8(18), P R O T A P(18)$, CANTAP $(17)$, CLTAPE $(16)$, I POCTAP (15), PLOTAP (14), SRFTAP (13), LIBTAP (12), CRDTAP( 11$)$, CORTAPI 9$)$,

TAPES1( 8$)$,TAPES $2(7)$, TAPES31 6$)$, TAPES $4(5)$,

C

FORTIN( 4). INTAPE( 3), IOUTAP( 2), PUNTAP( 1$)$ i

\section{EQUIVALENCE (KFLAGST 701 ,}

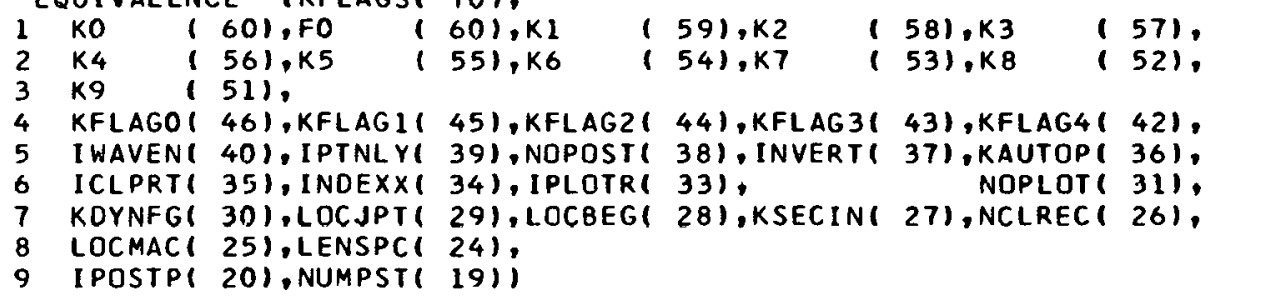

C

EQUIVALENCE (KOM (8), KAPTID(1),KAPTIO(2),KAPTTR(3),KAPTCN(4))

C

COMMON SYSTEM

C

DIMENSION KFLAGS(1), IPOSTP(1),KOM(1)

C

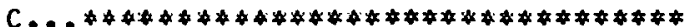

C COMMON AND DIMENSION-POSITIONING POST-PROCESSORS

C

COMMON MACH, NLOOP, NLOOP I, PNOFLG, MTABLE, NID, BI TS, BUFFER, COMSTO, 1CTRDLY,CTRFLG, FEDFLG, FEDIPR, FEDTAB, FORMAT, GGL, INTAP, IND2, IND3, $2 K O M E N T, K D O L, K X, K Y, K Z, L I N C T R, N A X I S, N C O O L, N F E E D C$, NPRINT, 3NODAT, NSPIN, NTEST, NTOOL 1, NTOOL2, NTRANS, NWPR, NZCAM, OPTAB, 4PRE 8U , PRESTX, PRESTY, PRESTZ, PREVX, PREVY, PREVZ, RADIUS, RETURN, 5RPM, SEQCTR, SFM, SP I FLG, SPNTAB, TAPSTO, TOOLNO, TRANSX, TRANSY, 6TR ANSZ, WORDS, BUFPRT, I DUM, ZCAM

DIMENSION MACH (12), PNOFL G( 14$)$, MTABLE( 10), BUFFER (50),COMSTO(13), IFEOTAB ( 50$)$, FORMAT (14), INTAP $(20)$, OPTAB (50), PREBU $(14)$, SPNTAB (100),

C 2TAPSTO(250), WORDS (14), BUFPRT (20), I DUM (50)

20130101 20130105 20130111 20130113 20130117 20130123 20130125 20132201 20130205 20130209

$N=M T A B L E(6)$

IFI IDUM $(18)-1) 27,8,27$

27 IF (OPTAB $(8)-1.0) 2,1,2$

1 IF(INTAP $(3)-3) 41,40,41$

41 PRESX $=O P T A B(2)$ 


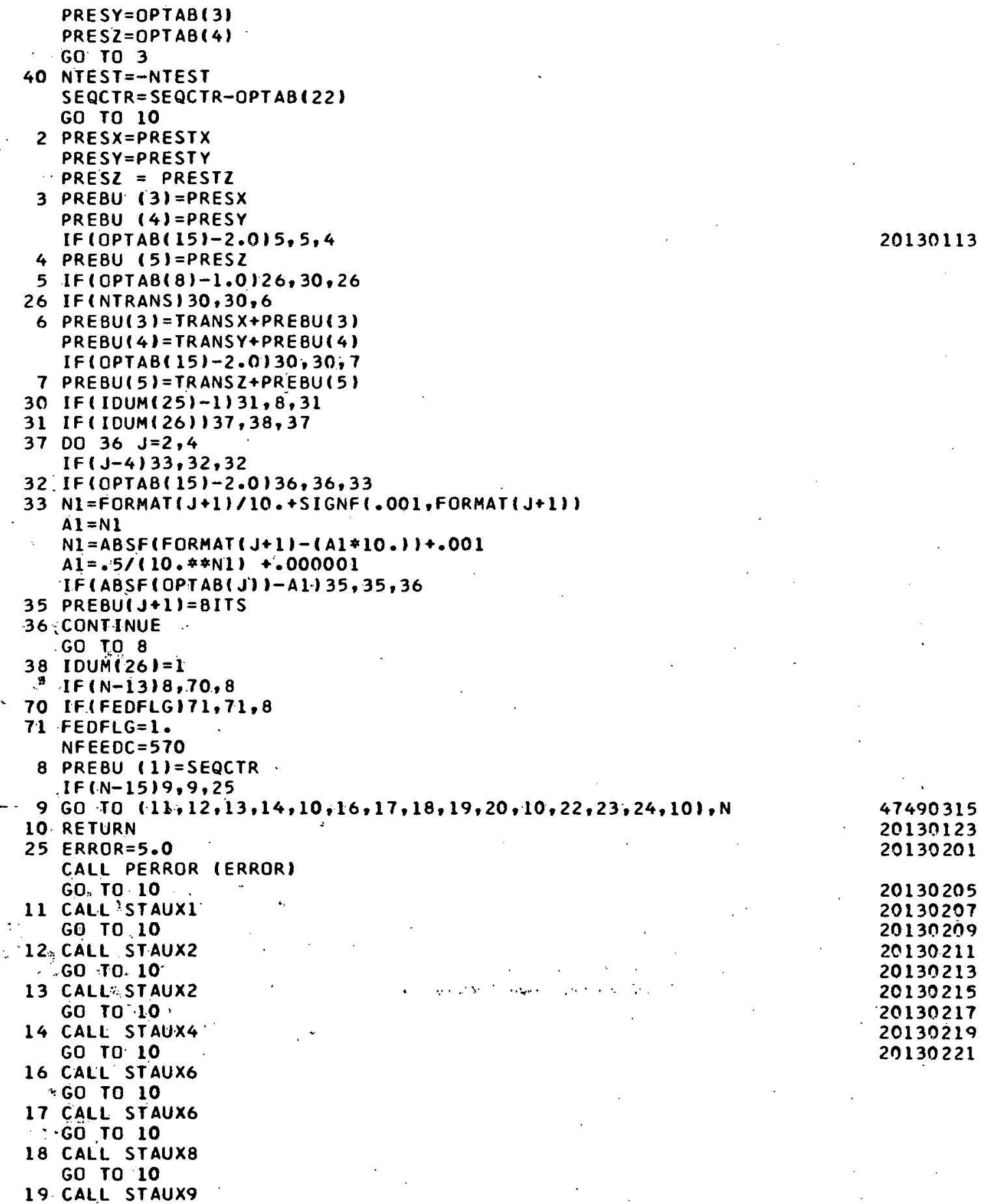


GO TO 10

20 CALL STAUIO

Go TO 10

22 CALL STAUI2

GO TO 10

23 CALL STAU13

GO TO 10

45720114

45720115

4 CALL STAUI4

GO TO 10

47490316

END

* Fortran

* LIST

* $\angle A B E L$

C5200 SPINCI

SUBROUTINE SPINCI

20130101

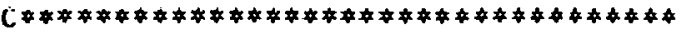

C... SYSTEM COMMON, DIMENSION, AND EQUIVALENC5+STATEMENTS

DIMENSION SYSTEM $(1700)$

EQUIVALENCE (SYSTEM(1700),KOM (1700),KFLAGS(1692),TAPETB(1622))

EQUIVALENCE (TAPETB(18), PROTAP (18), CANTAP (17),CLTAPE (16), POCTAP (15), PLOTAP (14), SRFTAP(13), LIBTAP (12), CRDTAP (11): CORTAP( 9$)$.

TAPES1( 8),TAPES2 $(7)$,TAPES3 61 , TAPES41 51 ,

FORTIN( 4$)$, INTAPE( 31 , IOUTAP( 21 , PUNTAP( 1$)$

C

EQUIVALENCE (KFLAGSI 701,
$\begin{array}{llll}K O & (60), F D & (60), K 1 \\ K 4 & (56), K 5 & (55), K 6\end{array}$
$(56), K 5$
$1591, K 2$
$(58), K 3$
$(57)$,
K9 (51),
KFLAGO ( 46), KFLAG1 451 , KFLAG2 ( 44), KFLAG3( 431 , KFLAG4( 421 ,
IHAVEN ( 40 ), IPTNLY( 39), NOPOST( 38 ), INVERT( 37), KAUTOP( 36$)$,
ICLPRT( 35), INDEXX (34), IPLOTRI 33), NOPLOT ( 31):
KOYNFG( 30$)$, LOCJPT ( 29), LOCBEG( 28$)$,KSECIN( 27), NCLREC ( 26),
LOCMAC ( 25), LENSPC( 24$)$.
9 IPOSTP( 20), NUMPSTI 19);

C

C

EQUIVALENCE IKOM

$(8), \operatorname{KAPTIO}(1), \operatorname{KAPTIO}(2), \operatorname{KAPTTR}(3), \operatorname{KAPTCN}(4))$

C

COMMON SYSTEM

C

DIMENSION KFLAGS(1), IPOSTP(1),KOM(1)

C . .

$C$ COMMON AND OIMENSION-POSITIONING POST-PROCESSORS

COMMON MACH, NLOOP, NLOOPI, PNOFLG, MTABLE, NID, BITS, BUFFER, COMSTO, ICTRDLY, CTRFLG, FEDFLG, FEDIPR, FEDTAB, FORMAT, GGL, INTAP, IND2, IND3, $2 K O M E N T, K O O L, K X, K Y, K Z, L I N C T R, N A X I S, N C O O L, N F E E D C$, NPRINT, 3 NODAT, NSPIN, NTEST, NTOOL 1 , NTOOL 2 , NTRANS, NWPR, NZCAM, OPTAB, 4PREBII ,PRESTX, PRESTY, PRESTZ, PREVX, PREVY, PREVZ, RAOIUS, RETURN, 5RPM, SEQCTR, SFM, SPIFLG, SPNTAB, TAPSTO, TOOLNO, TRANSX, TRANSY, 6TR ANSZ, WORDS, 8UFPRT, IDUM, ZCAM

DIMENSION MACH (12), PNOFLG $(14)$, MTABLE( 10), BUFFER (50), COMSTO 13$)$, 1FEDTAB (50), FORMAT ( 14), INTAP( 20), OPTAB (50), PREBU $(14)$, SPNTAB $(100)$, 2TAPSTO 250$)$, HORDS $(14), 8$ BFPRT $(20)$, I DUM(50)

20130101 20130105 20130111 20130113 20130117 20130123 20130125 20130201 20130205 20130209 
C

IF (RPM) $30,31,30$

$30 I=O P T A B(7)$

IF (RPM-SPNTAB( I ) )1,5,5

1 KOMENT $=9$

NSP IN = I

2 CALL COMENT

20130105

GO TO 24

3 RETURN

31 NSPIN $=0$

OPTAB (26) $=0$.

IF (MTABLE(6)-10)3,25,3

$5 I=0 P T A B(12) * O P T A B(11)-O P T A B(11)+O P T A B(7)+.1$

6 IFIRPM-SPNTAB(I) $10,4,7$

7 KOMENT $=10$

CALL COMENT

4 NSPIN $=$ I

GO TO 24

$10 K=O P T A B(7)$

$L=O P T A B(11)$

D015 J J K, I, L

IF (RPM-SPNTAB(J)) $20,15,15$

20130109

20130111

15 CONT INUE

$E R R O R=3.0$

CALL PERROR (ERROR)

20 . NSP IN $=J-L$

24 OPTAB (26) = SPNTAB (NSPIN)

25 CALL SPLCOD

GO TO 3

END

* . FORTRAN

* LIST

* LAB EL

C5300 SPINDL

SUBROUTINE SPINDL

20130117

20130119

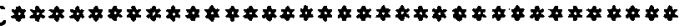

$C$

C... SYSTEM COMMON, DIMENSION, AND EQUIVALENC5+STATEMENTS

C

DIMENSION SYSTEM $(1700)$

C

c

EQUIVALENCE (SYSTEM(1700),KOM (1700),KFLAGS(1692), TAPETB(1622)

EQUIVALENCE (TAPETB $(18)$, PROTAP(18), CANTAP (17), CLTAPE (16),

1 POCTAP (15), PLOTAP (14), SRFTAP $(13)$, LIBTAP $(12)$, CROTAP 111 ),

2 CORTAP ( 9$)$,

3 TAPES1 $(8)$, TAPES $2(7)$, TAPES3 $(6)$,TAPES $4(5)$,

C

FORTINI 4), INTAPE( 3), IOUTAP( 2 ), PUNTAP ( 11$)$

equiVALENCE, (KFLAGSI 70),
KO 1601 , FO
( $601, K 1$
( 56),K5
( 55 ), K6
( 59$), \mathrm{K} 2$
$(58), K 3$
(57),
K9 (51),
( 54$), K 7$
( 53$), K 8$
(52),
KFLAGO (, 46), KFLAG1( 45), KFLAG2 ( 44), KFLAG3( 43), KFLAG4( 42), I WAVEN( 40 ), IPTNLY( 39), NOPOST ( 38), INVERT( 37), KAUTOP( 36 ), ICLPRT (35), INDEXX( 34), IPLOTRI 33), NOPLOT ( 31), KDYNFG( 30), LOCJPT( 29), LOCBEG( 28), KSECIN( 27), NCLREC( 26), LOCMACI 251, LENSPCI 241\%,
IPOSTPI 20I, NUMPSTI 19/) 
$\mathrm{C}$

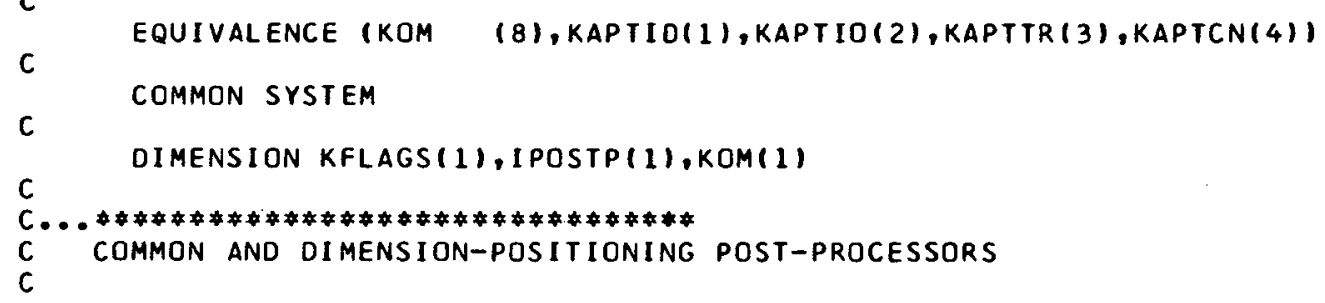

20130101 20130105 20130111 20130113 20130117 20130123 20130125 20130201 20130205 $2 n 13 n 2 n 9$

47490319

47490320

47490321

20130205

45720903

47490322

45720905

47490323

20130105

20130107

20130109

20130111

20130113

20130117 20130119

20130121

20130123

20130201

47490401 47490402 47490403 47490324 47490404 47490405 $474904 n 6$ 47490407 47490408 47490409 47490410 47490411 47490412

47490413 
26 TAPSTO $(L+1)=1$

47490414

19 IFITAPSTO(L+1)-DPTAB(1) $115,15,22$

47490415

15 OPTAB $(7)=$ TAPSTO $(L+1)$

47490416

GO TO 22

21 I $F(N W P R-(L+3)) 22,23,23$

23 IFI INTAP $(L+2)-145) 22,24,22$

24 IFI INTAP $(L+3)-61) 29,28,27$

27 IFI INTAP $(L+3)-63) 31,32,29$

47490417

47490418

47490419

47490420

28 TAPSTO $(L+3)=2$. GO TO 29

31 TAPSTO $(L+3)=3$. GO TO 29

32 TAPSTO $(L+3)=1$.

29 IFITAPSTO(L+3)-OPTAB(1) $125,25,22$

47490421

47490422

25 OPTAB $(7)=$ TAPSTO $(L+3)$

22 CALL SPINCI

IFI I DUM (23)) $34,30,34$

34. SPIIFLG $=0$.

PREBU $(10)$ i $=$ NSPIN

107 SEQCTR = SEQCTR+OPTAB (22) GO TO $(30,36,36,36,30,30,30,35,36,36,30,30,106,30,30), K$

47490423

47490424

47490425

20130209

35 PREBU(1) $=606000000000$ GO TO 37

106 PREBU $(14)=1$ DUM $(32)$.

36 PREBU ( 1 ) =SEQCTR

37 CALL CARD

CALL PRINT GO TO 30

30 RETURN

$33 N=O P T A B(19)+.1$ OPTAB $(7)=X M O D F(N, 2)+1$ GO TO 22

50 IFI INTAP(5)-115)5,51,1

51 SFM=TAPSTO(4)

IF ( INTAP (6)-23) $52,55,52$

20130217

20130219

20130221

52 IF(INTAP (8)-23)53,56,53

$53 \operatorname{IF}($ INTAP $(10)-23) 7,57,7$

55 RADIUS =TAPSTO $(7)$

GO TO 60

56. RADIUS $=$ TAPSTO $(9)$ GO TO 60

57 RADIUS= TAPSTO $(11)$ GO TO 60 .

60 RPM $=($ SFM*6.0)/(3.1.159*RADIUS)

GO TO 20

END

20130223

20130301

20130303

20130305

20130307

20130309

20130311

20130313

20130315

* Fortran

20130317

* : : LIST

* $\mathrm{ABEEL}$

C5400 SPLCOD

SUBROUTINE SPLCOD

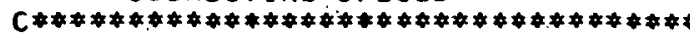

$C$

C... SYSTEM COMMON, DIMENSION, ANO EQUIVALENC5+STATEMENTS

C

C DIMENSION SYSTEM (1700)

C

EQUIVALENCE (SYSTEM(1700),KOM (1700),KFLAGS(1692), TAPETB(1622) 
COMMON MACH, NLOOP, NLOOP I, PNOFLG, MTABLE, NID, BITS, BUFFER, COMSTO, 1CTROL Y, CTRFLG, FEDFL G, FED IPR , FEDTAB, FORMAT, GGL, INTAP, IND2, IND3, 20130105 2KOMENT, KOOL, KX,KY,KZ, LINCTR, NAXIS, NCOOL, NFEEDC, NPRINT, 3NODAT, NSPIN, NTEST, NTOOL 1, NTOOL 2 , NTRANS, NHPR, NZCAM, OPTAB, 4PREBII , PRESTX, PRESTY, PRESTZ, PREVX, PREVY, PREVZ, RADIUS, RETURN, 5RPM, SEQCTR, SFM, SPIFLG, SPNTAB, TAPSTO, TOOLNO, TRANSX, TRANSY, 6TRANSZ, WORDS, BUFPRT, IDUM, ZCAM

DIMENSION MACH (12), PNOFLG $(14), M T A B L E(10)$, BUFFER $(50), C O M S T O(13)$, IFEDTAB (50), FORMAT (14), INTAP (20), OPTAB (50), PREBU (14), SPNTAB $(100)$,

C 2TAPSTO $(250)$, WORDS $(14)$, BUFPRT $(20)$, IDUM(50)

DIMENSION I SCODE (50)

$N T=M T A B L E(6)$

$N=N S P I N$

IF (NT-15) 2,2,1

1 RETURN

2 GO TO $(1,1,3,1,1,1,1,8,9,10,1,1,13,14,1)$ NT

47490501

$3 \operatorname{ISCODE}(1)=10$

$\operatorname{ISCODE}(2)=20$

I $S \operatorname{CODE}(3)=19$

I $\operatorname{SCOOE}(4)=29$

I SCODE $(5)=30$

I $\operatorname{SCODE}(6)=40$

I SCODE $(7)=50$

I SCODE $(8)=60$

$\operatorname{ISCODE}(9)=39$

I $\operatorname{SCODE}(10)=49$

I SCODE $(11)=59$

I $\operatorname{SCODE}(12)=69$

I $\operatorname{SCODE}(13)=70$

I SCODE $(14)=80$

I SCOOE $(15)=79$

I $S \operatorname{CODE}(16)=89$ 
$4 N S P I N=I S C O D E(N)$ GO TO 1

8 I $\operatorname{SCOOE}(1)=0$ I $S \operatorname{CODE}(2)=0$ I $\operatorname{SCODE}(3)=1$ I $\operatorname{COODE}(4)=1$ I $\operatorname{COODE}(5)=2$ I SCODE $(6)=2$ I SCODE $(7)=3$ I $S C O D E(8)=3$ I $\operatorname{SCODE}(9)=4$ I $S C O D E(10)=4$ I SCOOE $(11)=5$ I SCODE $(12)=5$ I $\operatorname{SCODE}(13)=6$ I SCODE (14) $=6$ I $S C O D E(15)=7$ I $\operatorname{SCODE}(16)=7$ GO TO 4

9 ISCOOE (1) $=530$ I SCODE $(2)=539$ I SCOOE (3) $=550$ I SCODE $(4)=566$ $1 S C O D E(5)=585$ I SCODE $(6)=611$ I SCODE $(7)=614$ I SCODE $(8)=619$ I SCODE $(9)=624$ I $\operatorname{SCODE}(10)=632$ I SCODE $(11)=641$ I $S C O D E(12)=653$ I SCODE (13) $=669$ I SCOOE (14) $=689$ ISCODE (15) $=712$ I SCODE $(16)=715$ GO 104

$13 \operatorname{ISCODE}(1)=1$ I $\operatorname{SCODE}(2)=2$ I SCOOE $(3)=3$ I SCODE $(4)=4$ ISCODE (5) = 5 I $S \operatorname{CODE}(6)=6$ I $\operatorname{SCODE}(7)=7$ I SCODE $(8)=8$ I $\operatorname{SCODE}(9)=9$ I $\operatorname{SCODE}(10)=10$ I $S C O D E(11)=11$ I $\operatorname{SCOOE}(12)=12$ I $S \operatorname{CODE}(13)=13$ I $\operatorname{SCODE}(14)=14$ I SCODE $(15)=15$ I SCODE $(16)=16$ $\operatorname{ISCODE}(17)=17$ I $S C O D E(18)=18$ $\operatorname{ISCODE}(19)=19$ I $\operatorname{SCODE}(20)=20$ I $S C O D E(21)=21$ I SCODE $(22)=22$ I SCODE $(23)=23$ 
I $\operatorname{SCODE}(24)=24$

I $\operatorname{SCODE}(25)=25$

I $\operatorname{SCOOE}(26)=26$

I $\operatorname{SCODE}(27)=27$

$1 \operatorname{SCODE}(28)=28$

$\operatorname{ISCODE}(29)=29$

$1 \operatorname{SCODE}(30)=30$

$1 \operatorname{SCODE}(31)=31$

I $\operatorname{SCODE}(32)=32$

GO TO 4

14 I $\operatorname{SCODE}(1)=00$

I $\operatorname{SCODE}(2)=37$

I $\operatorname{SCODE}(3)=11$

I $\operatorname{SCODE}(4)=38$

I $\operatorname{CODE}(5)=12$

I SCODE $(6)=39$

I $\operatorname{SCODE}(7)=13$

I $\operatorname{SCODE}(8)=40$

I $\operatorname{SCODE}(9)=14$

$1 \operatorname{SCODE}(10)=41$

$I S \operatorname{CODE}(11)=15$

I $\operatorname{SCODE}(12)=42$

I $\operatorname{SCODE}(13)=16$

I $\operatorname{SCODE}(14)=42$

$1 \operatorname{SCODE}(15)=17$

$\operatorname{ISCODE}(16)=42$

$I \operatorname{SCODE}(17)=18$

I $\operatorname{SCODE}(18)=42$

I SCONE $(19)=19$

$I \operatorname{SCODE}(20)=42$

I $S C O U E(21)=20$

I $S C O D E(22)=42$

I $\operatorname{SCODE}(23)=21$

I $\operatorname{SCODE}(24)=42$

I SCODE $(25)=22$

$1 \operatorname{SCODE}(26)=42$

$\operatorname{ISCODE}(27)=23$

I $\operatorname{SCODE}(28)=42$

I $\operatorname{SCODE}(29)=24$

I $\operatorname{SCODE}(30)=42$

I $\operatorname{SCODE}(31)=25$

I $\operatorname{SCODE}(32)=42$

I $\operatorname{SCODE}(33)=26$

I $\operatorname{SCODE}(34)=42$

I $\operatorname{SCODE}(35)=27$

$1 \operatorname{SCODE}(36)=42$

I SCODE $(37)=28$

I $\operatorname{SCODE}(38)=42$

I $\operatorname{SCODE}(39)=29$

I $\operatorname{SCODE}(40)=42$

I $\operatorname{SCODE}(41)=30$

I $\operatorname{SCODE}(42)=42$

$1 \operatorname{SCODE}(43)=31$

I SCODE $(44)=42$

I $\operatorname{CODE}(45)=32$

I $\operatorname{SCODE}(46)=42$

I SCÜUE $(47)=33$

$I \operatorname{SCODE}(48)=42$

I $\operatorname{SCODE}(49)=34$

47490502 47490503

47490504

47490505

47490506 47490507 47490508 47490509 47490510

47490511

47490512

47490513

47490514

47490515

47490516

47490517

47490518

47490519

47490520

47490521

47490522

47490523

47490524

47490525

47490601

47490602

47490603

47490604

47490605

47490606

47490607

47490608

47490609

47490610

47490611

47490612

47490613

47490614

47490615

47490616

47490617

47490618

47490619

47490620

47490621

47490622

47490623

47490624

47490625 


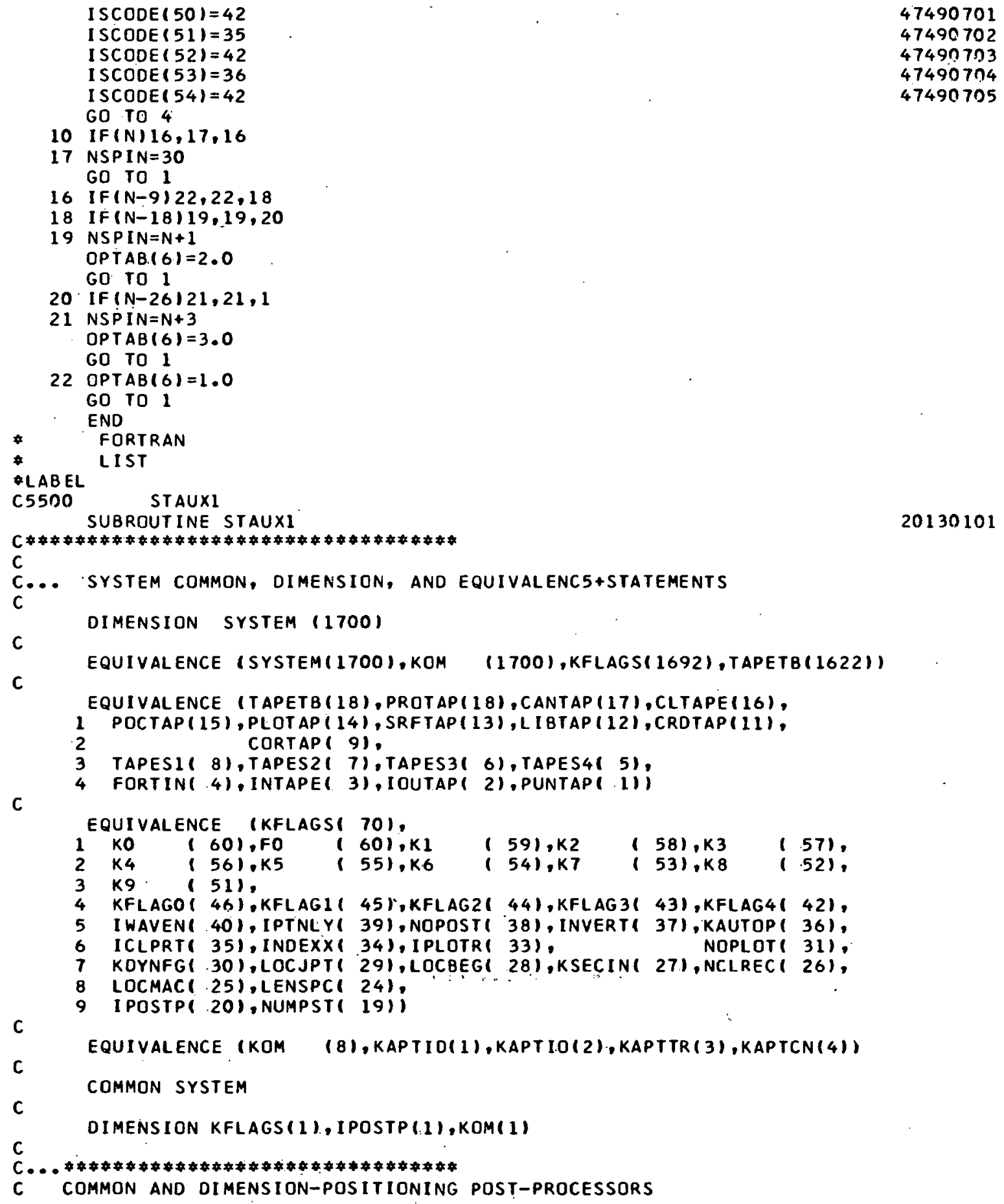


COMMON MACH, NLOOP, NLOOPI, PNOFLG, MTABLE,NID, BITS,BUFFER, COMSTO, 20130101 ICTRDLY, CTRFLG, FEDFLG, FEDIPR, FEDTAB, FORMAT, GGL, INTAP, IND2, IND3, 20130105 2KOMENT, KOOL, KX, KY, KZ, LINCTR, NAXIS, NCOOL, NFEEDC, NPRINT, 3NODAT, NSPIN, NTEST, NTOOL 1, NTOOL 2, NTRANS, NWPR, NZCAM, OPTAB, 20130111 4PREBU , PRESTX, PRESTY, PRESTZ, PREVX, PREVY,PREVZ, RAOIUS, RETURN, 5RPM, SEQCTR, SFM, SPIFLG, SPNT AB, TAPSTO, TOOLNO, TRANSX, TRANSY, 6TRANSZ, WORDS, BUFPRT, IDUM, ZCAM

DIMENSION MACH(12), PNOFLG(14), MTABLE(10), BUFFER (50), COMSTO (13), 20130123 20130125 IFEOTAB ( 50), FORMAT (14), INTAP (20), OPTAB (50), PREBU (14), SPNTAB (100), 20130205

C 2TAPSTO(250), WOROS (14), BUFPRT (20), I DUM(50)

IF(CTROLY) $1,2,1$

$1 \mathrm{GGL}=1.0$

PREBU $(2)=70.0$

GO TO 5

2 IF(GGL) $5,5,3$

$3 \mathrm{GGL}=0.0$ PREBU $(2)=0.0$

5 IF (NTOOL 1$) 6,10,6$

6 PREBU $(14)=$ TOOLNO NTOOL $1=0$ NTOOL $2=0$

10 RETURN END

* FORTRAN

LI ST

* LABEL

C5600 STAUX2

SUBROUTINE STAUX2

20130105

20130109

20130111

20130113

20130117

20130121

20130123

20130124

20130125

C

$C$

C... SYSTEM COMMON, DIMENSION, AND EQUIVALENC5+STATEMENTS

c DIMENSION SYSTEM (1700)

EQUIVALENCE (SYSTEM(1700),KOM (1700),KFLAGS(1692),TAPETB(1622))

EQU IVALENCE (TAPETB (18), PROTAP $(18)$, CANTAP (17), CLTAPE $(16)$,

1 POCTAP ( 15), PLOTAP (14), SRFTAP (13), LIBTAP (12), CROTAP (11),

2 CORTAP( 91,

TAPES1( 81 , TAPES2 $(7)$, TAPES3 61 ,TAPES4 51 ,

C

FORTIN( 4$)$, INTAPE( 3$),$ IOUTAP( 21 ,PUNTAP( 1$)$

EQUIVALENCE (KFLAGSI 70) 


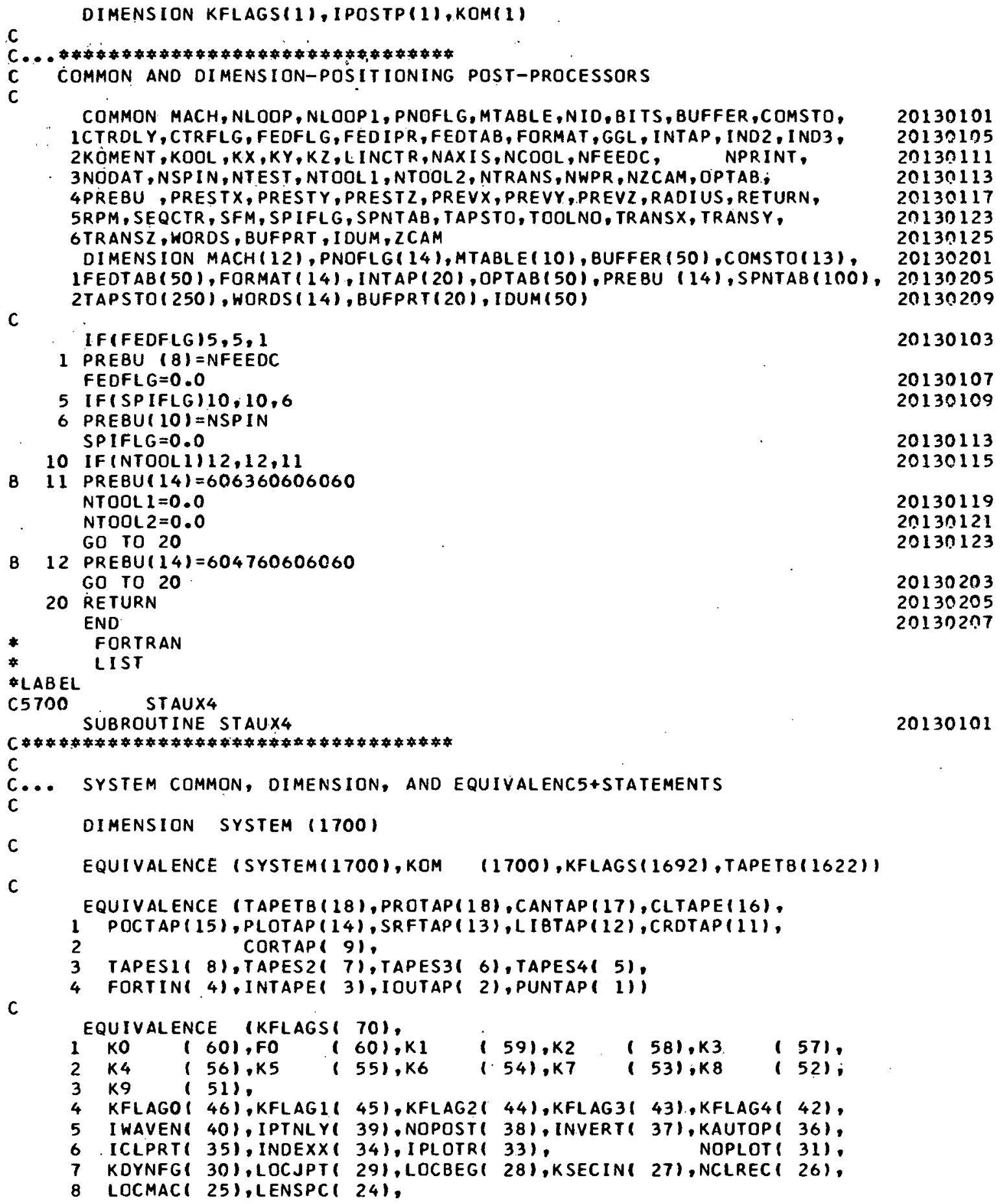


C

C

C

$\mathrm{C}$

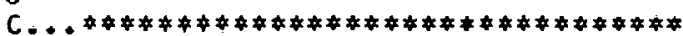

C COMMON AND DIMENSION-POSITIONING POST-PROCESSORS

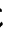

COMMON SYSTEM

DIMENSION KFLAGS(1), IPOSTP(1),KOM(1)

9 IPOSTP( 20), NUMPST( 19))

EQUIVALENCE (KOM (8),KAPTIDI1),KAPTIO(2),KAPTTR(3),KAPTCN(4))

COMMON MACH, NLOOP, NLDOP I, PNOFLG, MTABLE, NIO, BITS, BUFFER, COMSTO, 1CTRDLY,CTRFLG, FEOFLG, FEDIPR, FEDTAB, FORMAT, GGL, INTAP, IND2, IND3,

20130101 $2 K O M E N T, K O O L, K X, K Y, K Z, L I N C T R, N A X I S, N C O O L, N F E E D C$, NPRINT, 3NODAT, NSPIN, NTEST, NTOOL I, NTOOL 2 , NTRANS, NWPR, NZCAM, OPTAB, 4PREBU, PRESTX, PRESTY, PRESTZ, PREVX,PREVY,PREVZ, RADIUS, RETURN, 5RPM, SEQCTR, SFM, SP IFLG, SPNTAB, TAPSTO, TOOLNO, TRANSX, TRANSY. 6 TRANSZ, WORDS, BUFPRT, I DUM, ZCAM

DIMENSION MACH(12), PNOFLG(14), MTABLE( 10$), B U F F E R(50), C 0 M S T O(13)$, 1FEDTAB $(50)$, FORMAT $(14)$, INTAP $(20)$, OPTAB (50), PREBU $(14)$, SPNTAB (10O),

C 2TAPSTO(250), WORDS (14), BUFPRT (20), I DUM(50)

20130111

20130113

20130117

20130123

20130125

$201302 n 1$

20130205

20130209

IF (NTOOL 1$) 1,5,1$

20130103

1 IF (TOOLNO-1.0) 5,2,3

2 PREBU $(2)=1.0$

GO TO 4

20130105

3 PREBU $(2)=3.0$

4 NTOOL $1=0$

GO TO 10

5 I F (NTOOL 2$) 6,10,6$

6 IF (TOOLNO-1.0) 10.7 .8

7 PREBU $(2)=0.0$

GO TO 9

20130109

8 PREBU $(2)=2.0$

9 NTOOL2 $=0$

10 IF (NZCAM) $11,20,11$

11 GO TO 15

13 CALL PERROR (ERROR)

15 IF $(K O O L-1) 16,17,19$

16 TEMP $=0.0$ GO TO 18

17 TEMP $=10.0$

18 PREBU $(14)=T E M P+Z C A M$ GO TO 28

19 ERROR $=7.0$ GO TO 13

20 IF (NCOOL) $11,30,11$

28 N2CAM $=0$ $N C O O L=0$

30 IF (FEDFLG $140,40,31$

31 PREBU (8)=NFEEDC FEDFLG $=0.0$

20130113

20130115

20130117

20130119

20130123

20130203

20130205

40 IF(SPIFLG) $50,50,41$

41 PREBU $(10)=N 5 T I N$ SP I FLG $=0.0$

20130213

20130215

20130217

20130219

20130223

20130224

20130225

20130301

20130303

20130305

20130307

20130311

20130313

50 RETURN

END

20130317

20130319

$2 ? 139321$

* FORTRAN

* LIST 


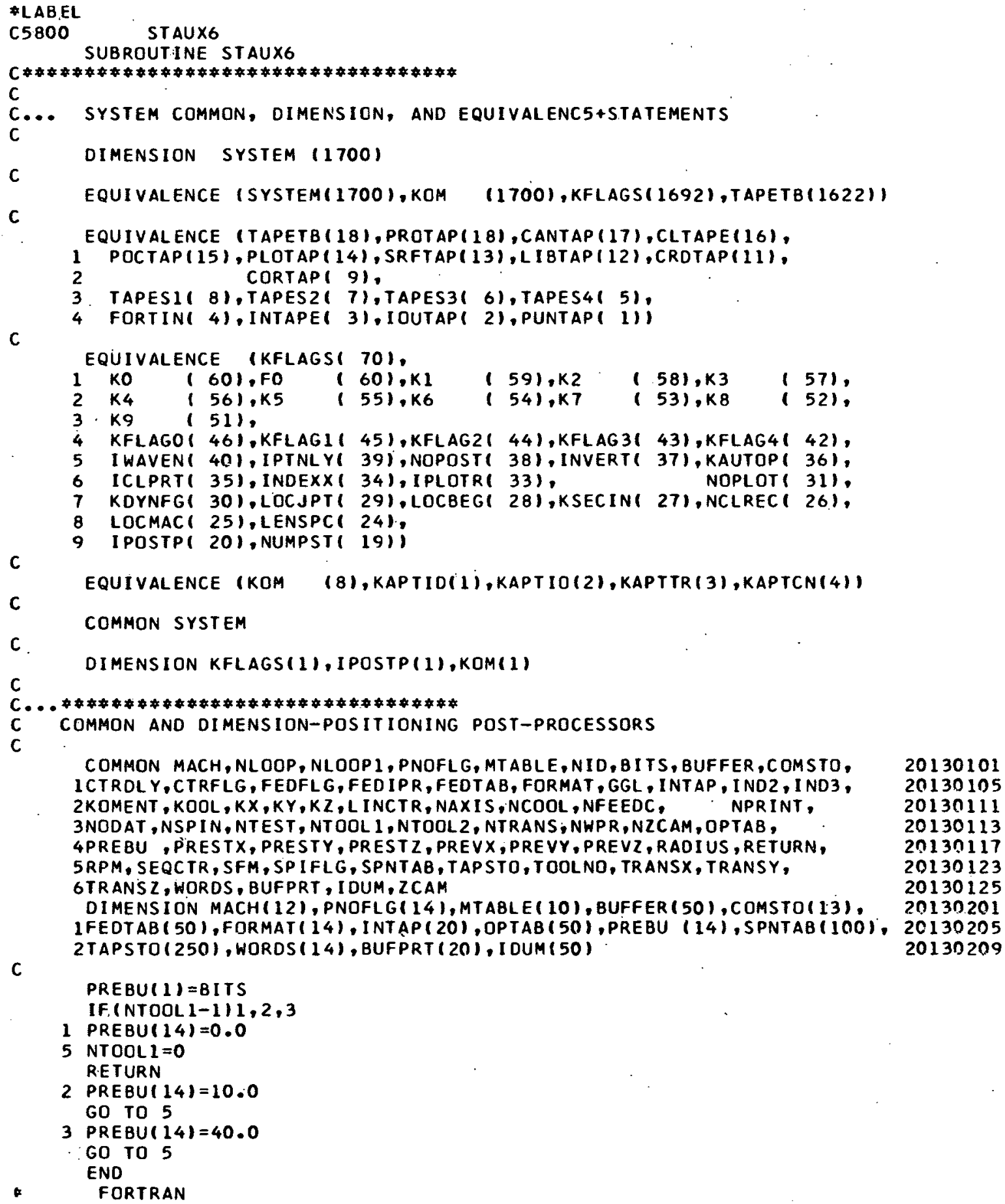

C

DIMENSION KFLAGS $(1), \operatorname{IPOSTP}(1), \operatorname{KOM}(1)$

C

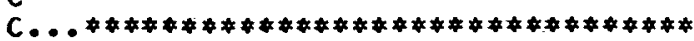

C COMMON ANO DIMENSION-POSITIONING POST-PROCESSORS

C

COMMON MACH, NLOOP, NLOOPI, PNOFLG, MTABLE,NID,BITS, BUFFER, COMSTO, ICTRDL Y, CTRFLG, FEDFLG, FED IPR, FEOTAB, FORMAT, GGL, INTAP, IND2, I ND3, 2KOMENT, KOOL, KX,KY,KZ, LINCTR, NAXIS, NCOOL, NFEEDC, NPRINT, 3NODAT, NSPIN, NTEST, NTOOL 1 , NTOOL2, NTRANS, NWPR, NZCAM, OPTAB, 4PREBU, PRESTX, PRESTY, PRESTZ, PREVX, PREVY, PREVZ, RADIUS, RETURN, 5RPM, SEQCTR, SFM, SPIFLG, SPNTAB, TAPSTO, TOOLNO, TRANSX, TRANSY, 6TRANSZ, WORDS, BUFPRT, IDUM, ZCAM

DIMENSION MACH (12), PNOFLG 141 , MTABLE(10),BUFFER(50), COMSTO (13). 1FEOTAB ( 50$)$, FORMAT 14$)$, INTAP (20), OPTAB (50), PREBU $(14)$, SPNTAB $(100)$, 2TAPSTO (250), WORDS $(14)$, BUFPRT $(20)$, I DUM(50)

20130101 20130105 20130111 20130113 20130117 20130123 20130125 20130201 20130205 20130209

C

PREBU (1) $=8$ ITS

IF. (NTOOL $1-1) 1,2,3$

1 PREBU $(14)=0.0$

5 NTOOLI $=0$ RETURN

2 PREBU $(14)=10.0$ GO TO 5

3 PREBU $(14)=40.0$ GO TO 5 END

* FORTRAN 


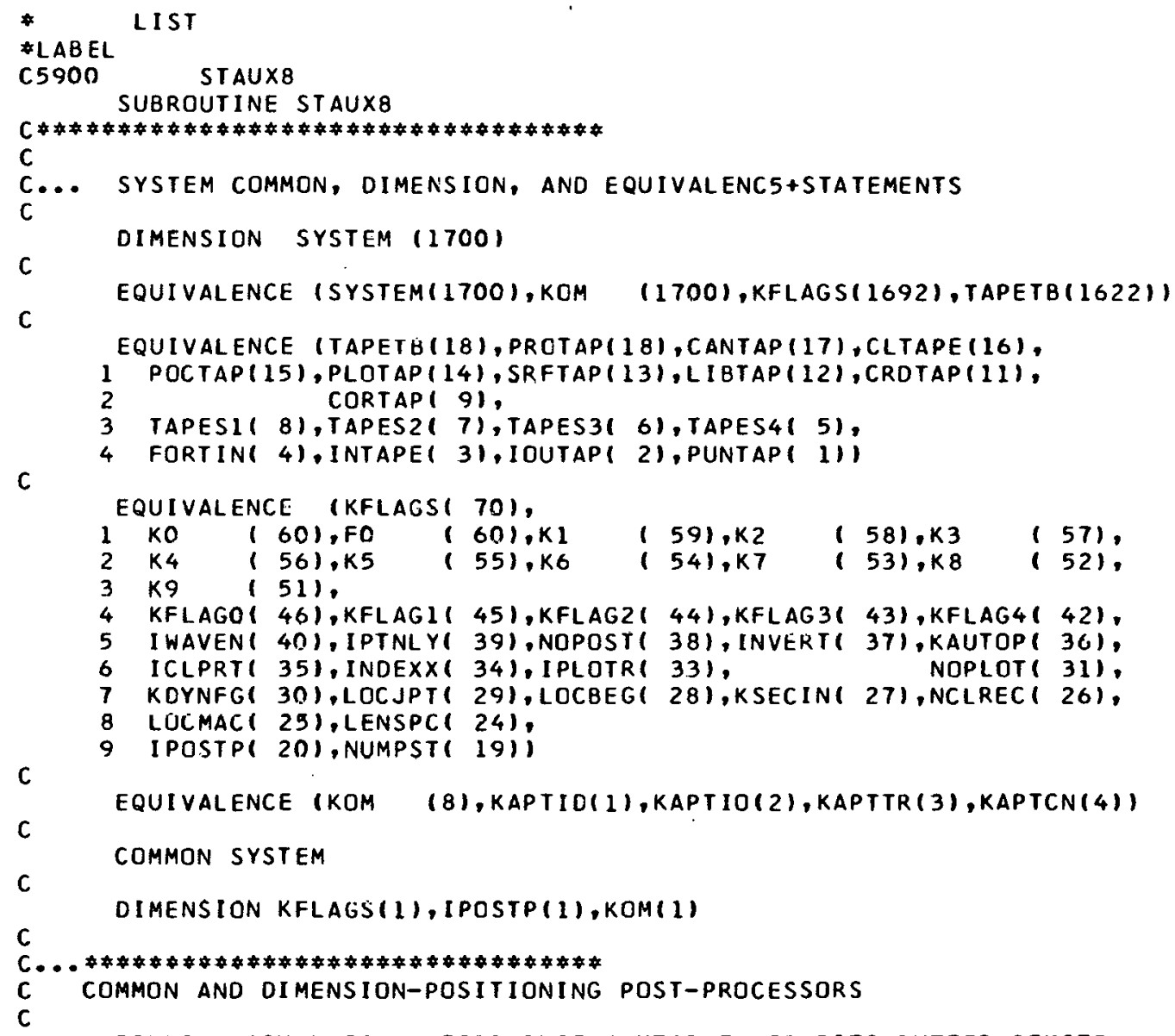

IF(FEDFLG) $5,5,1$

1 PREBU $(8)=N F E E D C$ FEDFLG $=0.0$

5 IF (SPIFLG) $10,10,6$

6 PRFRUI 10) $=$ NSP IN SPIFLG $=0.0$

B 10 PREBU(1) $=606000000000$

IF (NCODL) $11,1.2,11$

11 PREBU $(14)=9-\mathrm{KOOL}$ $\mathrm{NCOOL}=0$ 


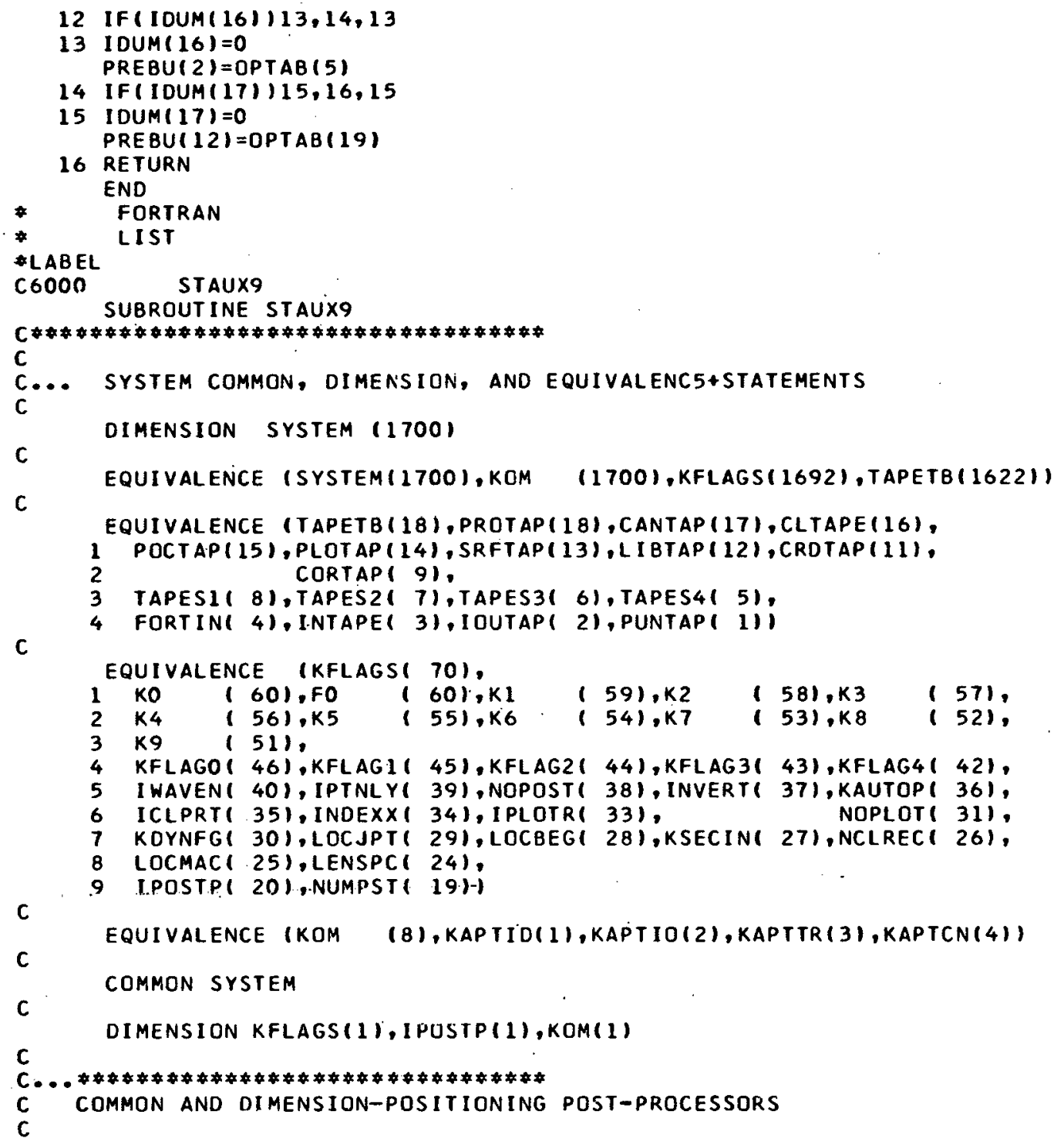

COMMON MACH, NLOOP, NLOOP 1, PNOFLG,MTABLE, NID,BITS,BUFFER,COMSTO, 20130101 1CTRDLY,CTRFLG, FEDFLG, FED IPR, FEDTAB, FORMAT, GGL ; INTAP, IND2, IND3, 20130105 2KOMENT, KOOL, KX, KY, KZ, LINCTR, NAXIS, NCOOL, NFEEOC, NPRINT; 3NODAT, NSPIN, NTEST, NTOOL 1 , NTOOL 2 , NTRANS, NWPR, NZCAM, OPTAB, 4PREBU. , PRESTX, PRESTY, PRESTZ, PREVX, PREVY, PREVZ, RADIUS, RETURN, SRPM, SEQCTR, SFM, SPIFLG, SPNTAB, TAPSTO, TOOLNO, TRANSX, TRANSY, GTRANSZ, WORDS, BUFPRT, IDUM, ZCAM

DIMENSION MACH (12), PNOFLG(14), MTABLE(10), BUFFER 150$),$ COMSTO (13), 1FEDTAB (.50), FORMAT ( 14$)$, INTAP (20), OPTAB (50), PREBU (14), SPNTAB (100),

C 2TAPSTO(250), HORDS $(14)$, BUFPRT $(20)$, I DUM(50)

20130111 20130113 20130117 20130123 20130125 $2013 n 201$ 20130205 20130209

IFIFEDFL $15,5,1$ 


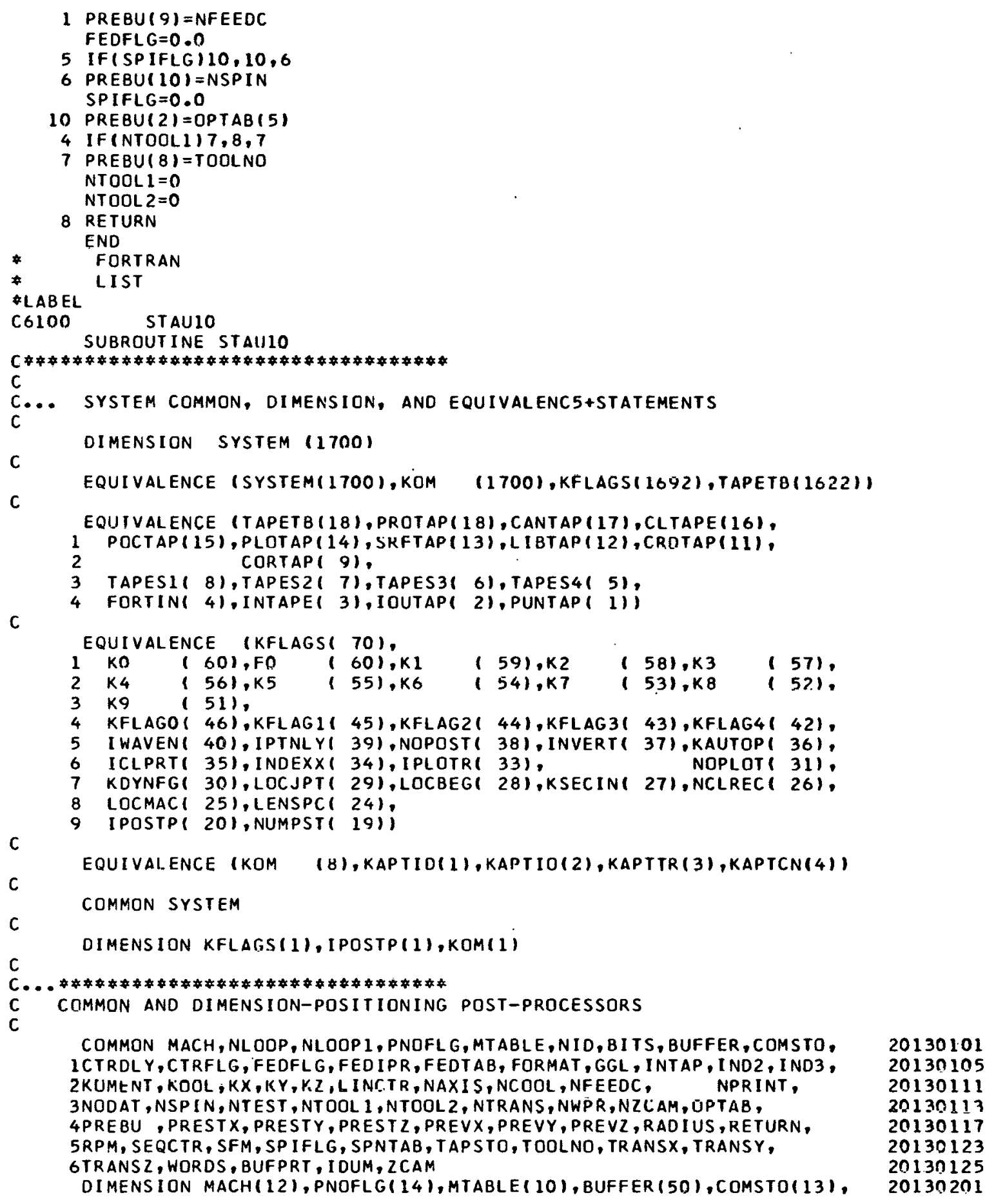


$c$ 2TAPSTO $(250)$, WORDS $(14)$, BUFPRT (20), IDUM(50)

IFIFEOFLG I5,5,1

1 PREBU $(8)=$ NFEEDC FEDFLG $=0.0$

5 IFISPIFLG) $10,10,6$

6 PREBU $(10)=N S P I N$ SPIFLG $=0.0$

10 IF (NCOOL I) 11,12,11

11 PREBU $(14)=9-K O O L$ $\mathrm{NCOOL}=0$

12 IFI IOUM(16) I13, 3,13

$13 \operatorname{IDUM}(16)=0$ PREBU $(2)=O P T A B(5)$

3. IFI I DUM ( 201$) 4,7,4$

B

4 PREBU (11) $=605160606060$ I DUM $(20)=0$

7 IFIIDUM( 18$)) 8,9,8$

$8 \operatorname{IDUM}(18)=0$ PREBU $(6)=0 P T A B(21)$

9 IFIIDUMI 19/115,16,15

$15 \operatorname{IDUM}(19)=0$ PREBU $(9)=$ OP TAB (24)

16 IF(IDUM(24)) 17,18,17

$17 \operatorname{IDUM}(24)=0$ PREBU ( 14) =OPTAB (25)

18 RETURN

END

*

* LABEL

C6200

FORTRAN

LIST

STAUI2

SUBROUTINE STAUI2

C

C.. SYSTEM COMMON, DIMENSION, AND EQUIVALENC5+STATEMENTS DIMENSION SYSTEM (1700)

C

C

EQUIVALENCE (SYSTEM(1700),KOM (1700),KFLAGS(1692),TAPETB(1622)

EQUIVALENCE (TAPETB $(18)$, PROTAP $(18)$, CANTAP $(17)$, CLTAPE $(16)$, 1 POCTAP (15), PLOTAP (14), SRFTAP( 13$),$ L IBTAP (12), CRDTAP (11), CORTAP( 9$)$.

TAPESI $(8)$,TAPES $2(7)$, TAPES3 $(6)$, TAPES4 $(.5)$,

C.

FORTIN( 41 , INTAPE 31 , IOUTAPI 21 , PUNTAP 113

EQUIVALENCE IKFLAGSI 70 ).
KO (60),FO
( $601, K 1$
$(56), K 5$
$(551 . K 6$
$1591, \mathrm{~K} 2$
$(58), K 3$
$(57)$
$\begin{array}{lll}K 4 & (56), K 5 \\ K 9 & (51) \text {, }\end{array}$
KFLAGO ( 46), KFLAG1 ( 45), KFLAG2 ( 44$)$, KFLAG3 ( 43), KFLAG4 ( 42), I WAVEN ( 40), IPTNLY( 39), NOPOST( 381 , INVERT( 37 ), KAUTOP ( 36): ICLPRT ( 35), INDEXX (34), IPLOTR 33), KDYNFG( 30$)$, LOCJPT (29), LOCBEG( 281, KSECIN( 27$)$, NCLREC $(26)$, LOCMAC ( 25), LENSPC( 24), IPOSTP( 20), NUMPST( 19)

C 


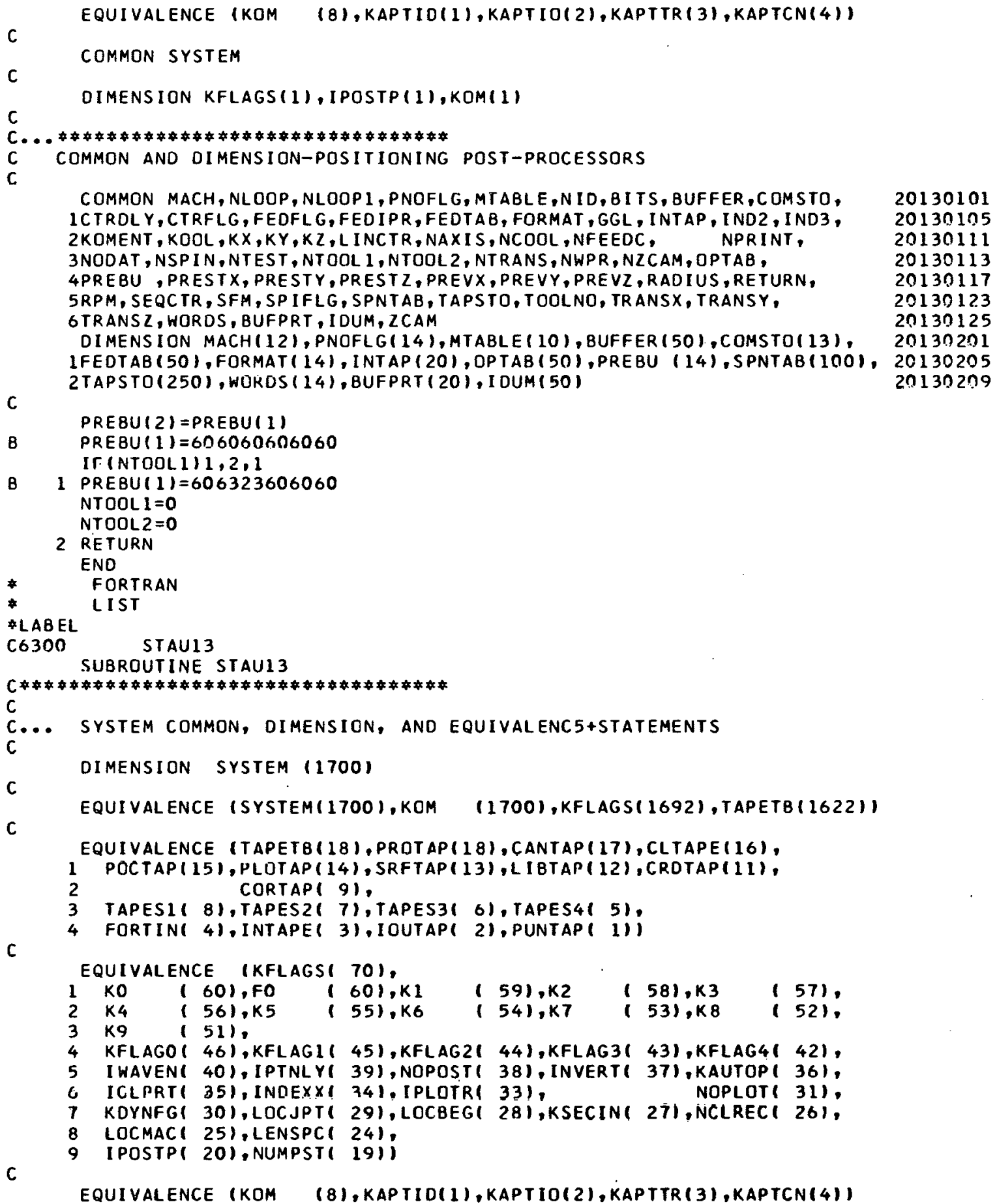




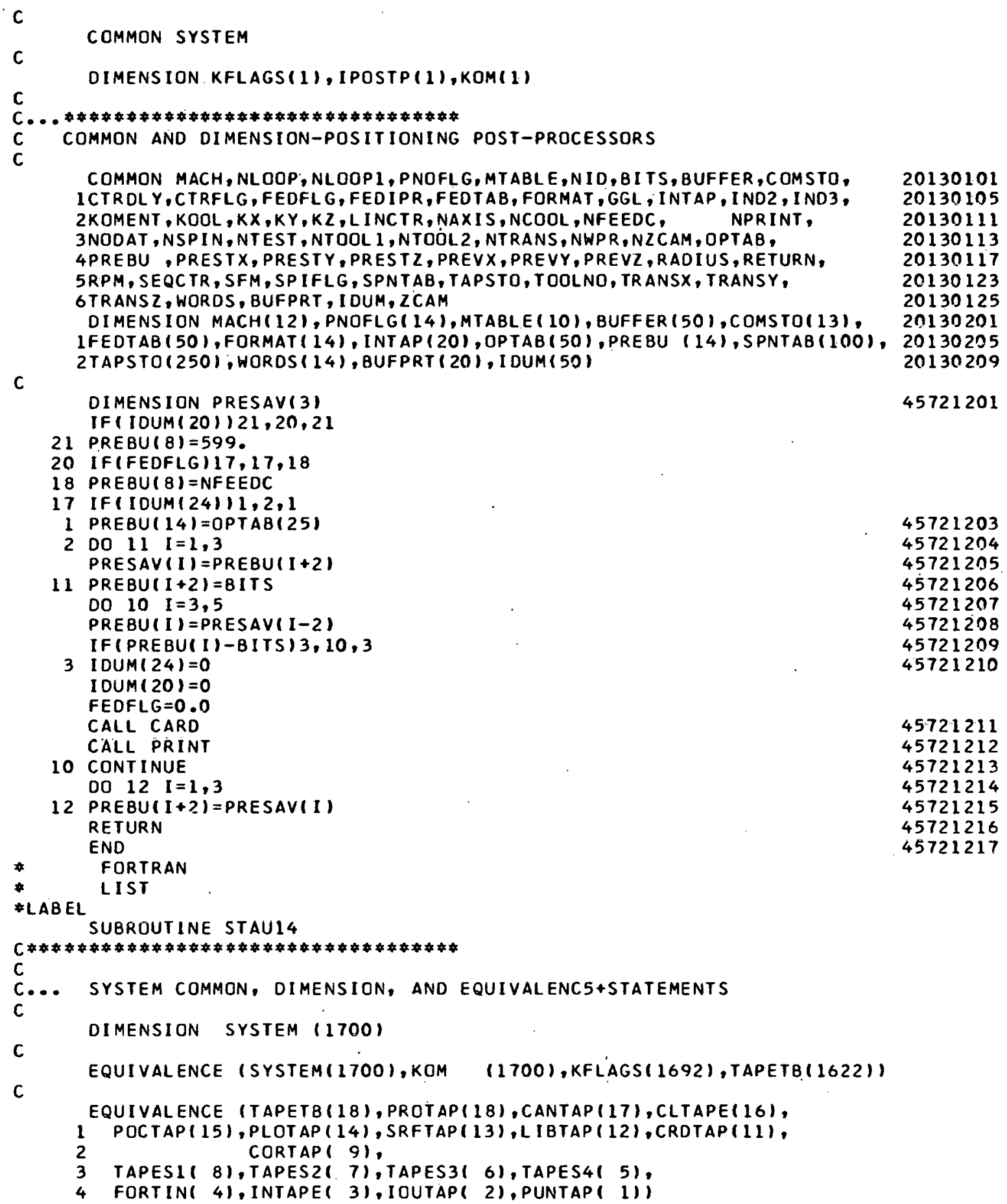


C

equivalence (KFLAGS( 70$)$,

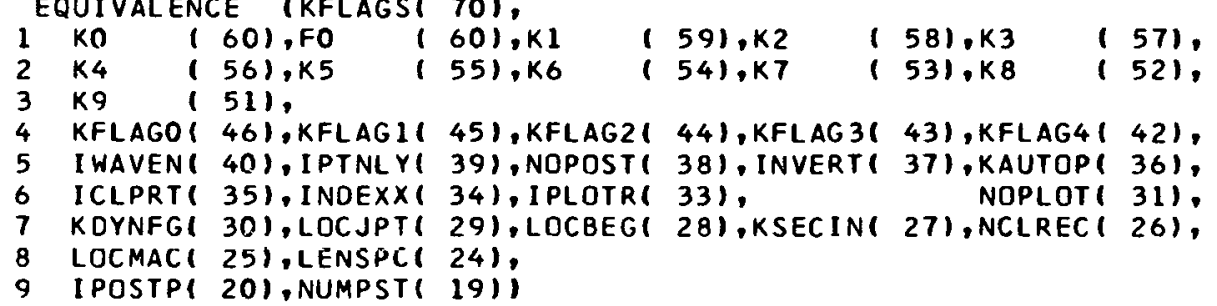

C

C

EQUIVALENCE (KOM (8),KAPTID(1),KAPTIO(2), KAPTTR(3),KAPTCN(4))

C

COMMON SYSTEM

C

DIMENSION KFLAGSI 1$)$, IPOSTP(1),KOMI 1 )

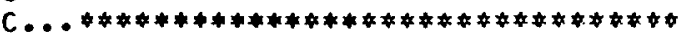

C COMMON AND DIMENSION-POSITIONING POST-PROCESSORS

C

C

COMMON MACH, NLOOP, NLOOP 1 , PNOFLG, MTABLE, NID, BITS, BUFFER, COMSTO, 1CTROL Y.CTRFLG, FEOFLG, FEDIPR, FEOTAB, FORMAT, GGL, INTAP, IND2, IND3, 2KOMENT, KOOL, KX, KY, KZ, L INCTR, NAXIS, NCOOL, NFEEOC, NPRINT, 3NDDAT, NSP IN, NTEST, NTOOL 1 , NTOOL 2 , NTRANS, NWPR, NZCAM, OPTAB, 4PREBU, PRESTX, PRESTY, PRESTZ, PREVX, PREVY, PREVZ, RADIUS, RETURN, SRPM, SEQCTR, SFM, SP I FLG, SPNTAB, TAPSTO, TOOLNO, TRANSX, TRANSY, 6TRANSZ, WIRDS, BUFPRT, IDUM, ZCAM

DIMENSION MACH (12), PNOFLG (14), MTABLE (10), BUFFER (50), COMSTO (13), IFEDTAB (50), FORMAT ( 14), INTAP (20), OPTAB (50), PREBU (14), SPNTAB $(100)$, 2 TAPSTO $(250)$, WORDS $(14)$, BUFPRT $(20)$, I DUM $(50)$

20130101 20130105 20130111 20130113 20130117 20130123 20130125 20130201 20130205 $2013 n 209$

DIMENSION SAVIT(3)

$N=I D U M(3 n)-1$

$M=I D U M(30)$

I $F(M-1) 16,5,6$

6 IF(IDUM( 33$)) 28,28,29$

$29 \operatorname{IDUM}(33)=M$

$28007 \quad I=1, N$

IF(NCOOL-I) $8,9,8$

9 PREBU $(14)=9-\mathrm{KOOL}$

NCONL $=0$

GO TO 10

8 IF(IUUM(31)-I $111,12,11$

$12 \operatorname{IOUM}(31)=0$

IF ( IDUM(32)-I SAVE) I5,7,15

15 PREBU $(14)=$ IDUM (32)

I SAVE = IDUM( 321

GO TO 10

$11 \operatorname{IF}(\operatorname{IDUM}(24)-1) 7,14,7$

14 PREBU (14) =OPTAB (25) $\operatorname{IDUM}(24)=0$

10 DO $13 \quad I=1,3$ SAVIT(I)=PREBU $(I+2)$

13 PREBU $(I+2)=8$ ITS

CALL CARD

CALL PRINT

PREBU $(1)=$ SEQCTR

DO $26 \quad I=1,3$
47490608 47490609 47490610

47490611

47490612

47490614

47490615

47490617

47490618

47490619

47490622

47490623

47490624

47490625

47490702 47490704 


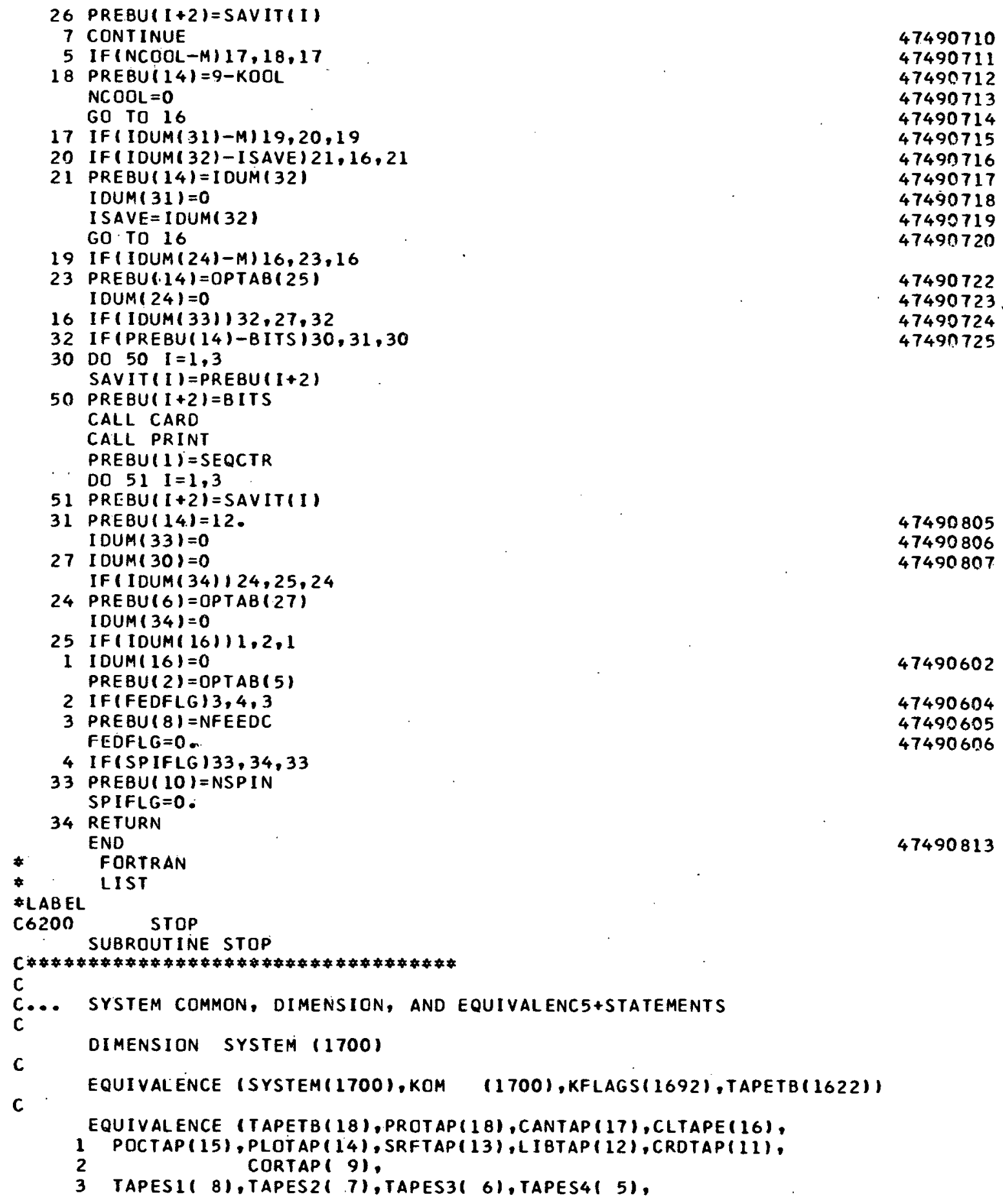


C

4 FORTIN( 4 ), INTAPEI 3), IOUTAPI 21 ,PUNTAP 111 EQUIVALENCE (KFLAGS( 70$)$

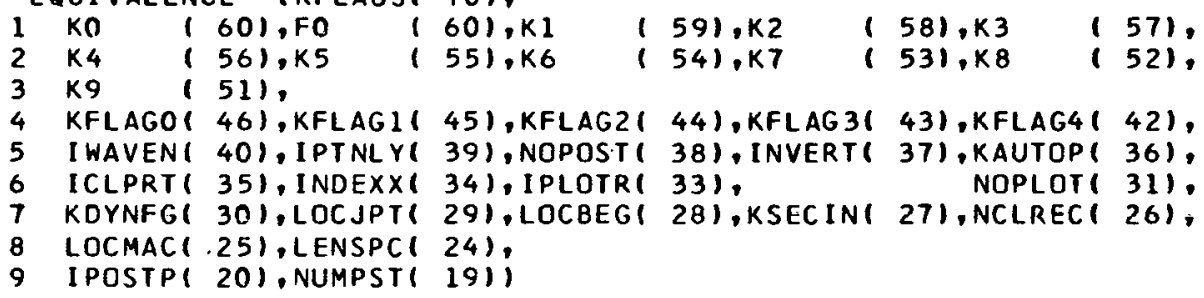

C

EQUIVALENCE (KOMM (8), KAPTID(1), KAPTIO(2), KAPTTR(3), KAPTCN(4))

C

COMMON SYSTEM

C

C

DIMENSION KFLAGS(1), IPOSTP(1),KOM(1)

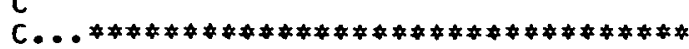

C COMMON ANO DIMENSION-POSITIONING POST-PROCESSORS

C

COMMON MACH, NLOOP, NLOOPL, PNOFLG, MTABLE, NID, BITS, BUFFER, COMSTO, 1CTRDLY, CTRFLG, FEDFLG, FED IPR, FEDTAB, FORMAT, GGL, INTAP, IND2, IND3, $2 K O M E N T, K O O L, K X, K Y, K Z, L I N C T R, N A X I S, N C O O L, N F E E D C$, NPRINT, 3NODAT, NSPIN, NTEST, NTOOL 1, NTOOL2, NTRANS, NWPR, NZCAM, OPTAB, 4PREBU ,PRESTX, PRESTY, PRESTZ, PREVX, PREVY, PREVZ, RADIUS, RETURN, 5RPM, SEQCTR, SFM, SPIFLG, SPNTAB, TAPSTO, TOOLND, TRANSX, TRANSY, GTRANSZ, WOROS, BUFPRT, IOUM, ZCAM

DIMENS ION MACH(12), PNOFLG $(14)$, MTABLE (10), BUFFER (50),COMSTO(13), IFEOTAB (50), FORMAT (14), INTAP (20), OPTAB (50), PREBU $(14)$, SPNTAB $(100)$,

C 2TAPSTO(250), WOROS $(14)$, BUFPRT $(20)$, I DUM(50)

20130101 20130105 20130111 20130113 20130117 20130123 20130125 20130201

20130205 20130209

FEOFLG $=0$.

NCOOL $=0$

NTOOLI $=0$

NTOOL2 $=0$

SPIFLG $=0$.

$\operatorname{IDUM}(16)=0$

$\operatorname{IDUM}(17)=0$

$\operatorname{IDUM}(18)=0$

IDUM $(19)=0$

$\operatorname{IDUM}(20)=0$

$\operatorname{IDUM}(24)=0$

$N=M T A B L E(6)$

IF $(\mathrm{N}-15) 2,2,1$

2 GO TO $1,1,1,1,1,1,1,8,9,10,11,1,10,10,1), N$

8 SEQCTR $=S E Q C T R+U P T A B(22)$

PREBU ( 1$)=606000000000$

PREBU (14) $=12$.

GO TO 20

9 PREBU $(2)=0$. $O P T A B(5)=0$.

$21 S E Q C T R=S E Q C T R+O P T A B(22)$

PREBU ( 1 ) = SEQCTR

GO TO 20

10 PREBU $(14)=0$.

GO TO 21

$11 \operatorname{PREBU}(8)=0$. 


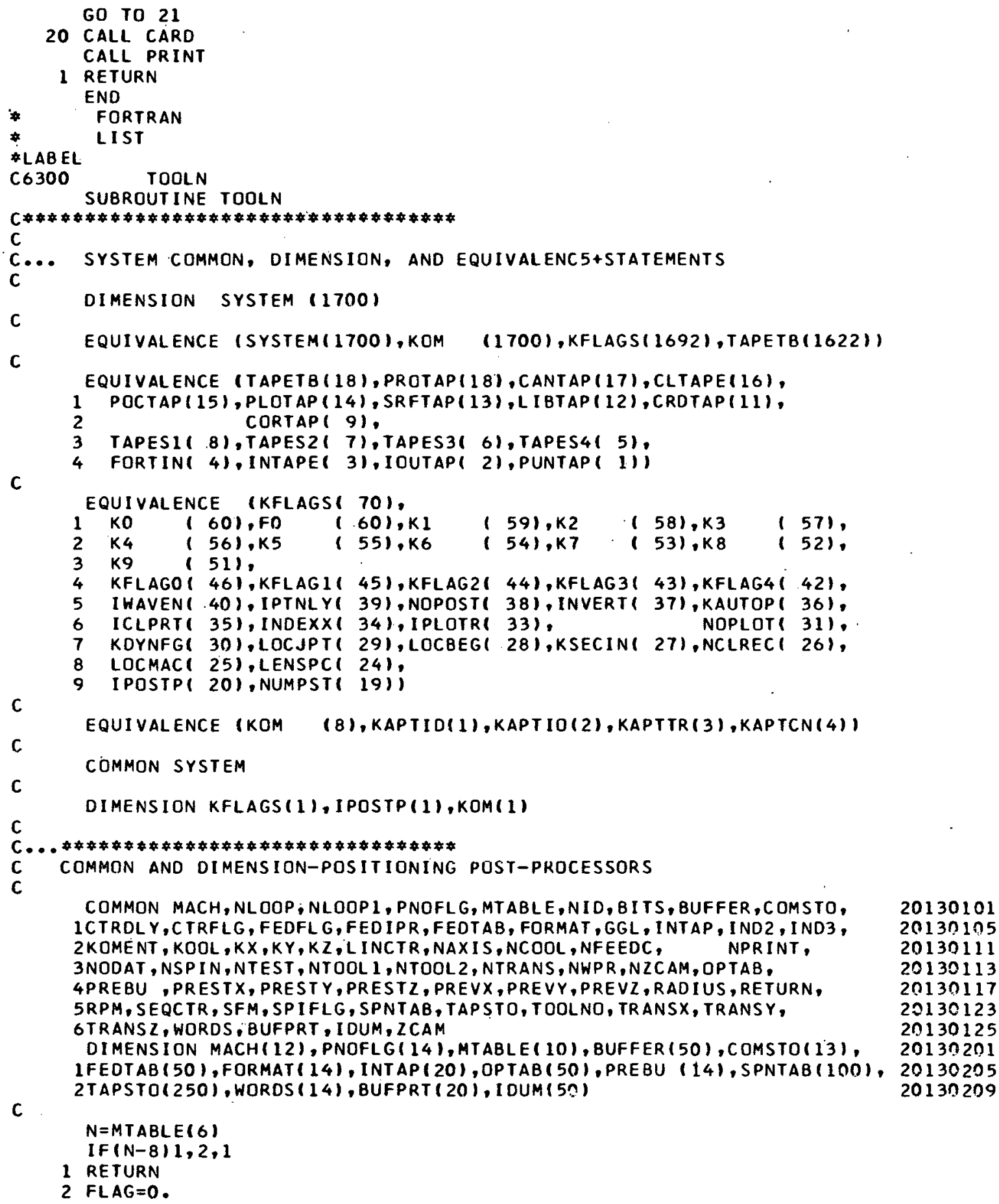

C

$$
N=M T A B L E(6)
$$




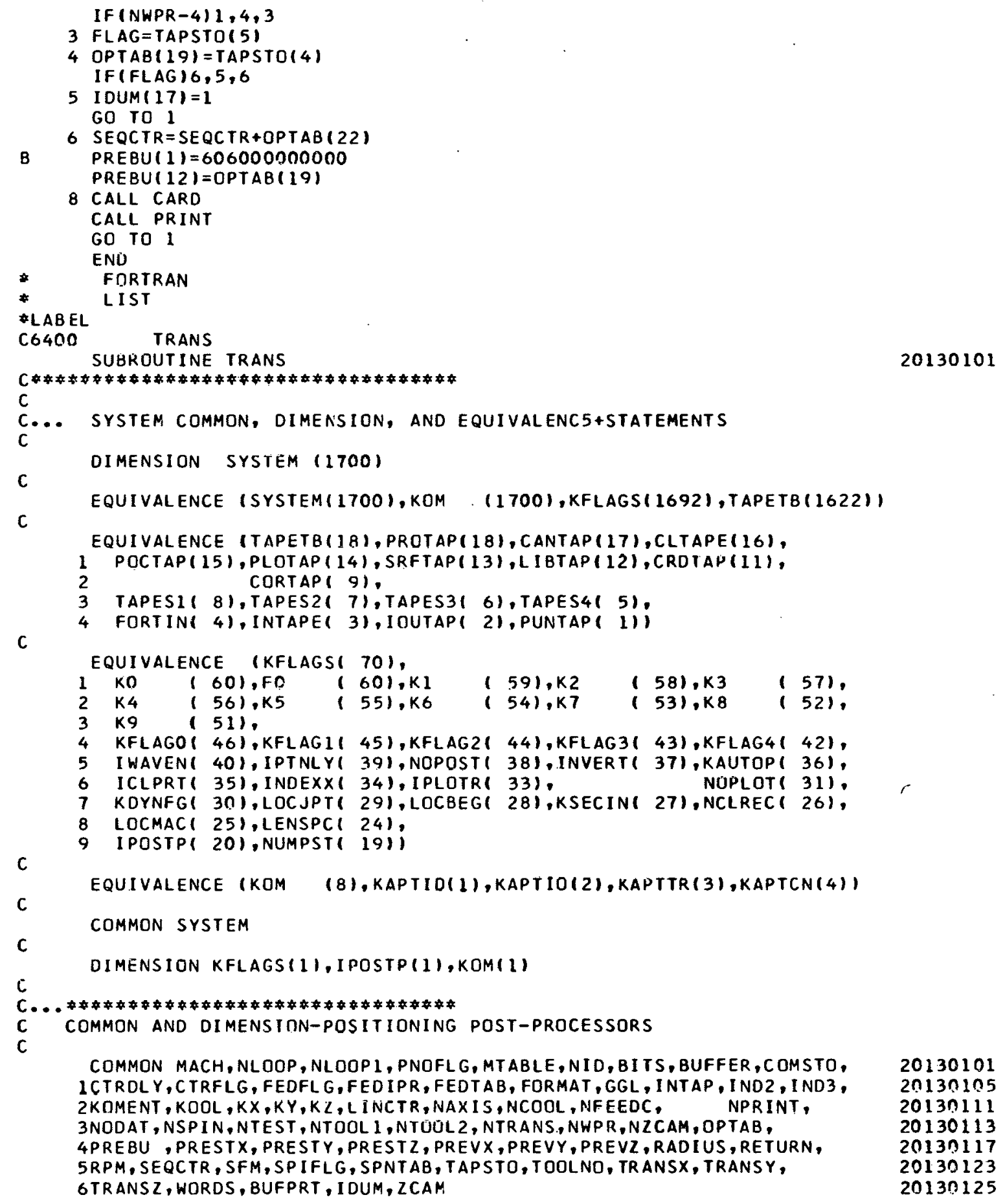

COMMON MACH, NLOOP, NLOOPI, PNOFLG, MTABLE, NID, BITS, BUFFER, COMSTO, ICTRDLY,CTRFLG, FEDFL G, FEDIPR, FEDTAB, FORMAT, GGL, INTAP, INO2, IND3, 2KOMENT, KOOL, KX, KY, KZ, L INCTR, NAXIS, NCOOL, NFEEDC, NPRINT, 3NODAT, NSPI N, NTEST, NTOOL 1 , NTUULL 2, NTRANS., NHPR, NZCAM, OPTAB, 4PREBU, PRESTX, PRESTY, PRESTZ, PREVX, PREVY, PREVZ, RADIUS, RETURN, 5RPM, SEQCTR, SFM, SPIFLG, SPNTAB, TAPSTO, TOOLNO, TRANSX, TRANSY, 6TRANSZ, WORDS, BUFPRT, IDUM, ZCAM 


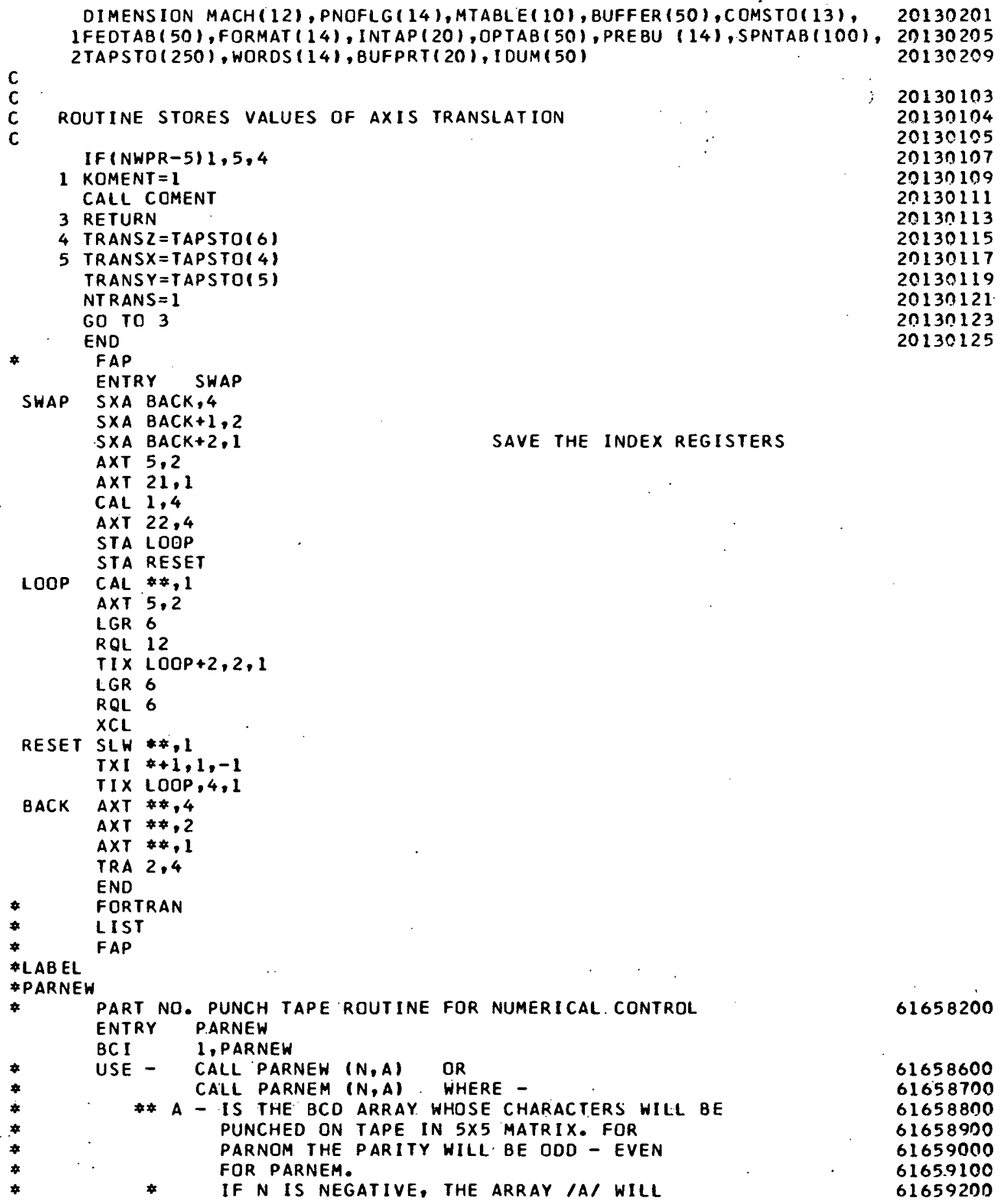




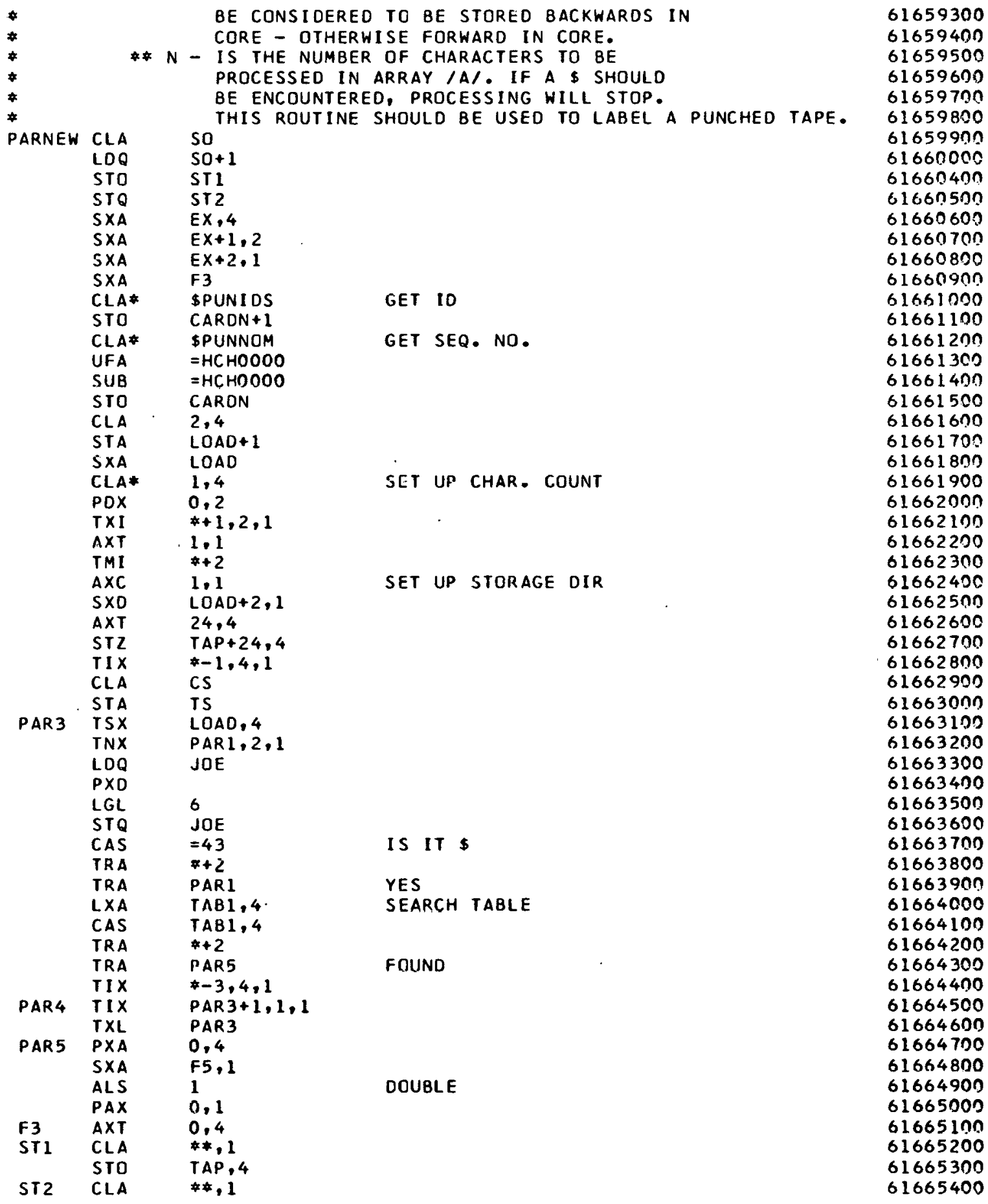




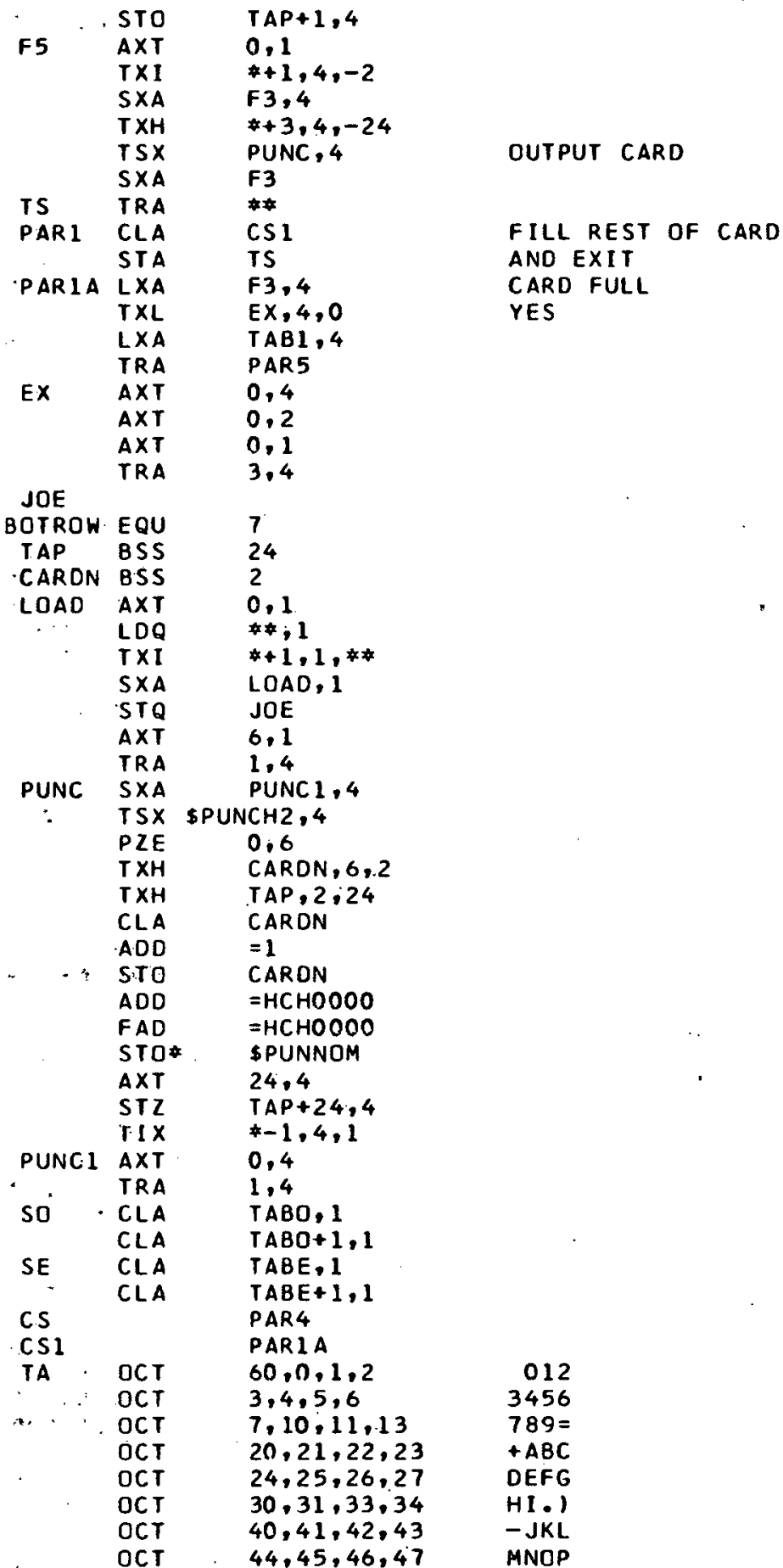

61665500

61665600

$6166570 n$

61665800

61665900

61666000

61666100

61666200

61666300

61666400

61666500

61666600

61666790

61666800

61666900

61667000

61667109

61667200

61667300

$616674 n n$

61667500

61667600

- 61667700

61667800

61667900

61668 inno

61668100

$616682 n n$

61668300

61668400

61668500

61668600

61668700

61668800

$616689 n 9$

61669000

61669100

.61669200

.61669300

61669400

61669500

61669600

61669702

$6166980 n$

61669900

61670000

61670100

61670200

61670300

61670400

61670500

61670600

61670700

616708 nn

61670900

61671000

61671100

61671200

$616713 n 0$ 


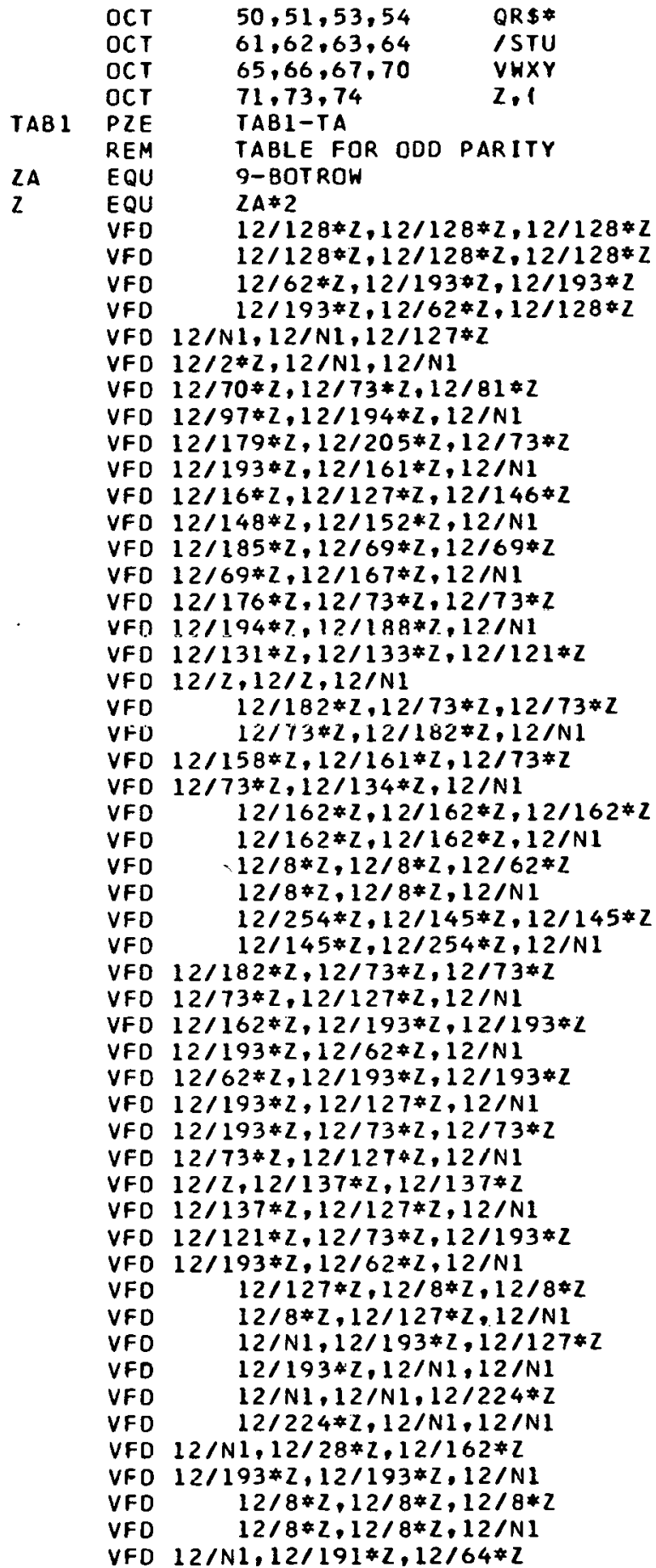

61671400

61671500 61671600

61671700

$6167180 n$

61671900

61672000

61672100

BL61672200

BL61672300

61672400

1

1

2

3

4

4

5

6

6

7

861674000 861674100 9

$=61674400$

$=61674500$

$+61674600$

$+61674700$

A61674800

A6167490n

B

B

C

C

D

E

E

$F$

G

H61676200

H61676300

I 61676400

161676500

.61676600

.61676700 i

)

$-61677000$

$-61677109$ 
VFD $12 / 64 * 2,12 / 32 * Z, 12 / N 1$

VFD $12 / 193 * Z, 12 / 162 * Z, 12 / 148 * Z$

VFD $12 / 8 * Z, 12 / 127 * Z, 12 / N 1$

VFD $12 / 64 * Z, 12 / 64 * Z, 12 / 64 * Z$

VFD $12 / 64 * Z, 12 / 127 * Z, 12 / N 1$

VFD $\quad 12 / 127 * Z, 12 / 2 * Z, 12 / 140 * Z$

VFD $\quad 12 / 2 * Z, 12 / 127 * Z, 12 / N 1$

VFD $12 / 127 * Z, 12 / 8 * Z, 12 / 4 * Z$

VFD $12 / 2 * 2,12 / 127 * 2,12 / N 1$

VFD $\quad 12 / 62 * Z, 12 / 193 * Z, 12 / 69 * Z$

VFD $\quad 12 / 67 * Z, 12 / 62 * Z, 12 / N 1$

VFD $12 / 134 * Z, 12 / 137 * Z, 12 / 137 * Z$

VFD $12 / 137 * 2,12 / 127 * 2,12 / N 1$

VFD $12 / 94 * Z, 12 / 161 * Z, 12 / 81 * Z$

VFD $12 / 193 * Z, 12 / 62 * Z, 12 / N 1$

VFD $12 / 70 * Z, 12 / 41 * Z, 12 / 25 * Z$

VFD $12 / 137 * Z, 12 / 127 * Z, 12 / N 1$

VFD $12 / 49 * 2,12 / 73 * 2,12 / 127 * 2$

VFD $12 / 73 * 2,12 / 70 * 2,12 / N 1$

VFD $\quad 12 / 8 * Z, 12 / 42 * Z, 12 / 28 * Z$

VFO $\quad 12 / 42 * Z, 12 / 8 * Z, 12 / N 1$

VFD $12 / 2 * Z, 12 / 4 * Z, 12 / 8 * Z$

VFD $12 / 16 * Z, 12 / 32 * Z, 12 / N 1$

VFD $12 / 49 * Z, 12 / 73 * Z, 12 / 73 * Z$

VFD $12 / 73 * Z, 12 / 70 * Z, 12 / N 1$

VFD $\quad 12 / 2,12 / 2,12 / 127 * Z$

VFD $\quad 12 / Z, 12 / Z, 12 / N 1$

VFD $\quad 12 / 191 * Z, 12 / 64 * Z, 12 / 64 * Z$

VFD $\quad 12 / 64 * 2,12 / 191 * 2,12 / N 1$

VFD $\quad 12 / 143 * Z, 12 / 176 * Z, 12 / 64 * Z$

VFD $\quad 12 / 176 * Z, 12 / 143 * Z, 12 / N 1$

VFD $\quad 12 / 191 * Z, 12 / 64 * Z, 12 / 188 * Z$

VFD $\quad 12 / 64 * 2,12 / 191 * Z, 12 / N 1$

VFD $\quad 12 / 227 * Z, 12 / 148 * Z, 12 / 8 * Z$

VFD $\quad 12 / 148 * 2,12 / 227 * 2,12 / N 1$

VFD $\quad 12 / 7 * Z, 12 / 8 * Z, 12 / 112 * Z$

VFD $\quad 12 / 8 * Z, 12 / 7 * Z, 12 / N 1$

VFD $12 / 67 * Z, 12 / 69 * Z, 12 / 73 * Z$

VFD $12 / 81 * Z, 12 / 97 * Z, 12 / N 1$

VFD $12 / N 1,12 / 176 * Z, 12 / 208 * Z$

VFD $12 / N 1,12 / N 1,12 / N 1$

VFD $12 / N 1,12 / 193 * Z, 12 / 193 * Z$

VFD $12 / 162 * Z, 12 / 28 * Z, 12 / N 1$

TABO BSS 0

$K 1$ EQU $192 * 2$

N1 EQU $128 * 2$

REM TABLE FOR EVEN PARITY

YFO $12 / K 1,12 / K 1,12 / K 1$

VFO $12 / \mathrm{Kl}, 12 / \mathrm{Kl}, 12 / \mathrm{Kl}$

VFD $\quad 12 / 190 * Z, 12 / 65 * Z, 12 / 65 * Z$

VFD $\quad 12 / 65 * Z, 12 / 190 * Z, 12 / K 1$

VFD $\quad 12 / K 1,12 / 130 * Z, 12 / 255 * Z$

VFD $12 / K 1,12 / K 1,12 / K 1$

VFD $\quad 12 / 66 * Z, 12 / 225 * Z, 12 / 209 * Z$

VFD $\quad 12 / 201 * Z, 12 / 198 * 2,12 / K 1$

VFD $\quad 12 / 33 * Z, 12 / 65 * Z, 12 / 201 * Z$

VFD $\quad 12 / 77 * 2,12 / 51 * Z, 12 / K 1$

VFO $\quad 12 / 24 * 2,12 / 20 * 2,12 / 18 * Z$

VFD $\quad 12 / 255 * Z, 12 / 144 * Z, 12 / K 1$

$J$
$k$
$K$
$L$

61677800 61677900

N

$N$

061678200

061678300

$\mathbf{P}$

Q.

R

R

$\$$

*61679200

*61679300

1

s

$S$

T61679800

T61679900

U6168000n

U61.680100

V616802n0

V61 $6803 n n$

H61680400

H61680500

$\times 61680600$

$\times 61680700$

Y 61680800

Yo1680900

$\mathbf{Z}$

Z

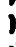

61681600

61681700

61681800

61681900

BL6168200n

BL61682100

61682200

61682300

$161682.4 \mathrm{nn}$

161682500

261682600

261682700

361682800

361682900

461683000

461683100 

661683500 761683600 761683700 $86168380 n$ 861683900 961684000 961684100 $=61684200$ $=616843 n n$ $+61684400$ $+61684500$ A61684600 A6168470n $B 61684800$ B61684900 C61685000 C61685100 61685200 061685300 61685400 E61685500 F61 1685600 F6168570n G61685800 G61685900 H61686000 H616861no $1616862 n 0$ I 61686300 .6168640 n .61686500 $16168660 n$ 161686700 $-61686800$ -6168690 n $J 61687000$ J6168710? K61687200 K6168730? L61687400 161687500 $616876 n 0$ 61687700 N61687800 N61687900 061688000 061688100 P61688200 P6168830n 061688400 Q61688500 R61688600 R61688700 $\$ 61688800$ $\$ 61688900$ *61689000 


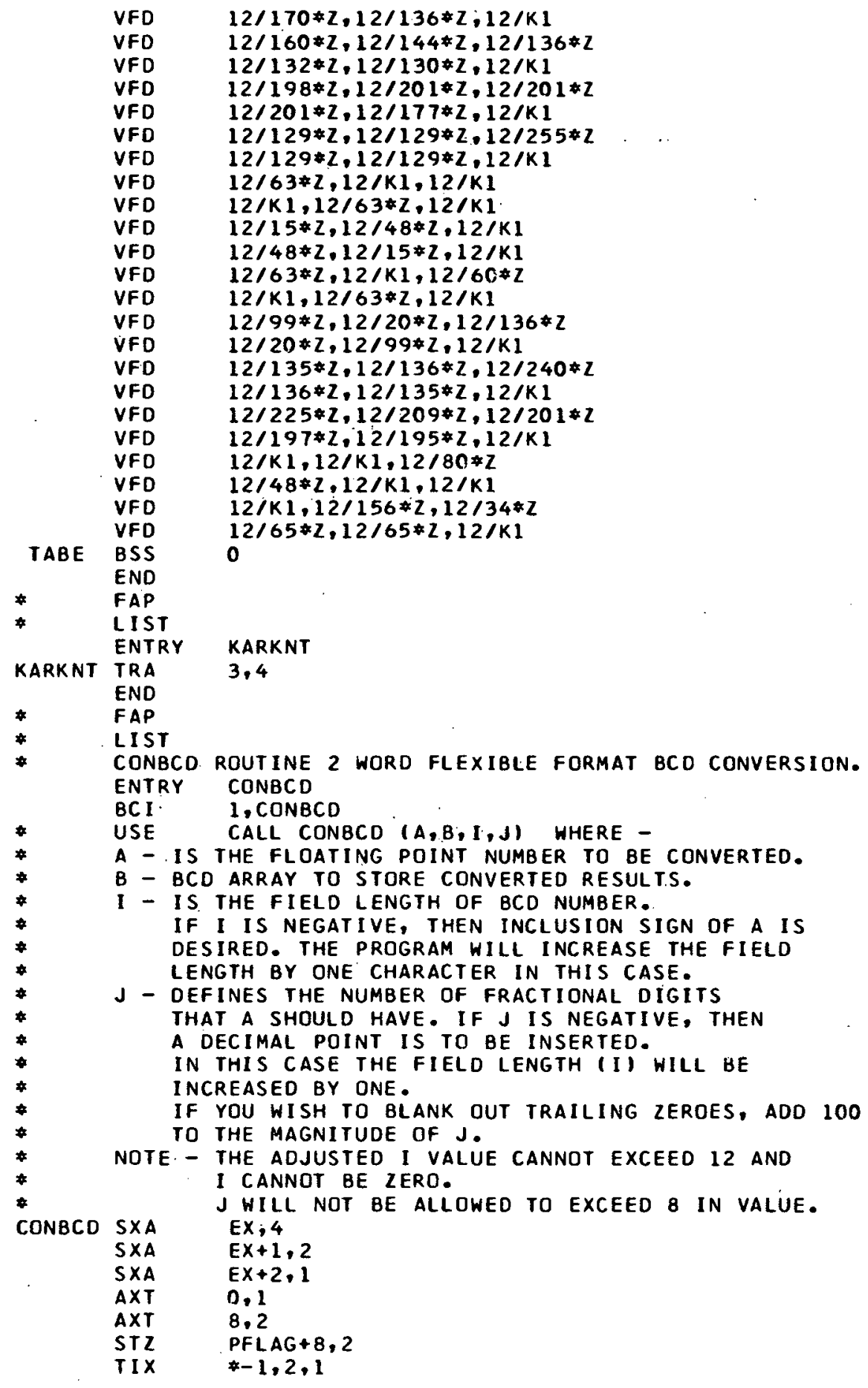

$\$ 61689100$

161689200

$16168930 n$

561689400

S6168950n

T61689600

T6168970n

U61689800

U61689900

V616900nn

V61690100

W61690200

$W 61690300$

$\times 61690400$

$\times 61690500$

61690600

Y61690700

Z61690800

261690900

.61691000

, 61691100

$616912 \mathrm{nn}$

161691300

$616914 n 0$

61691500

61409600

61409700

61409800

61409900

61410000

61410100

61410200

61410300

61410400

61410500

61410600

61410700

61410800

61410900

61411000

61411100

61411200

61411300

61411400

$6141150 ! 0$

61411600

61411700

61411800

61411900

61412000

61412100

61412200 


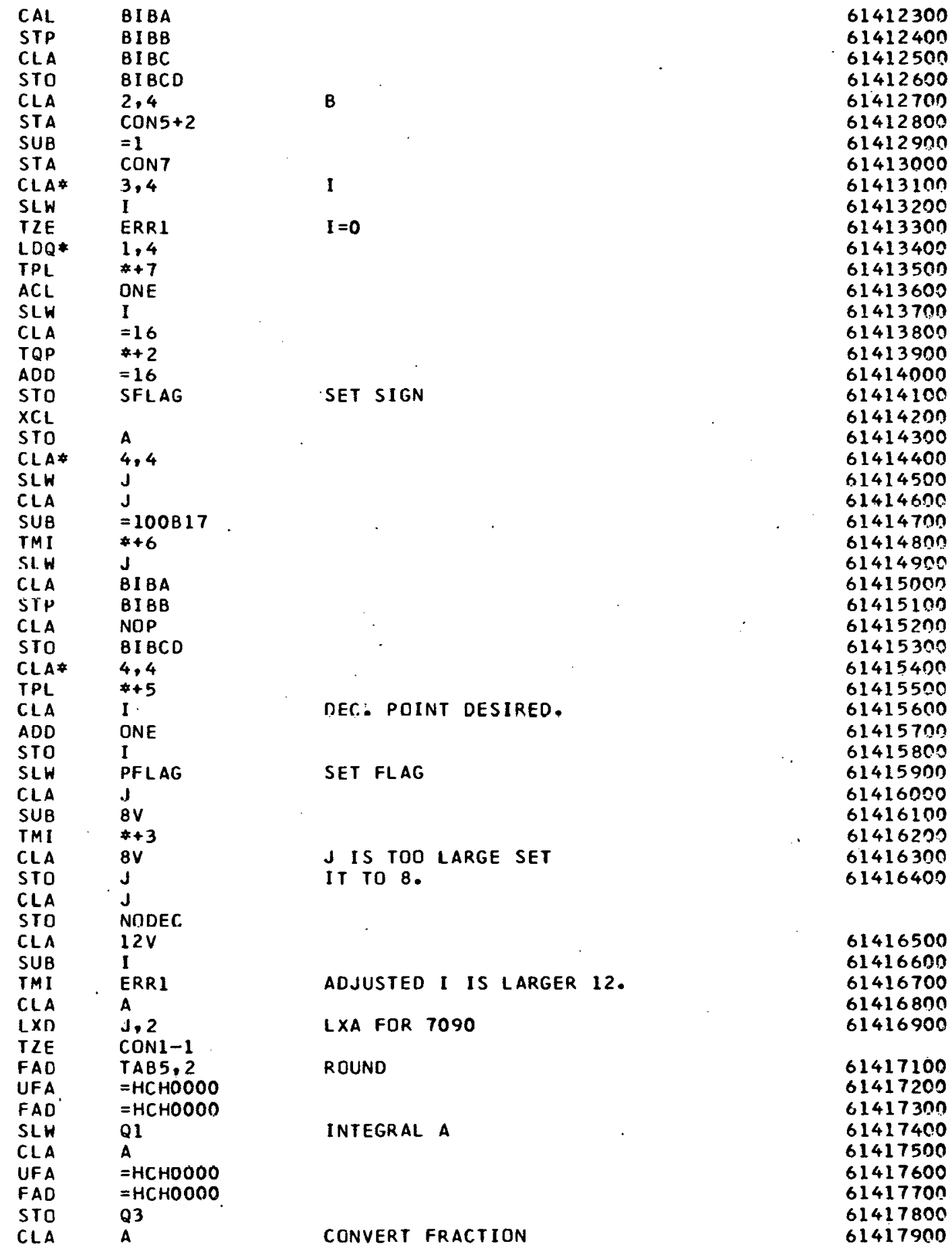




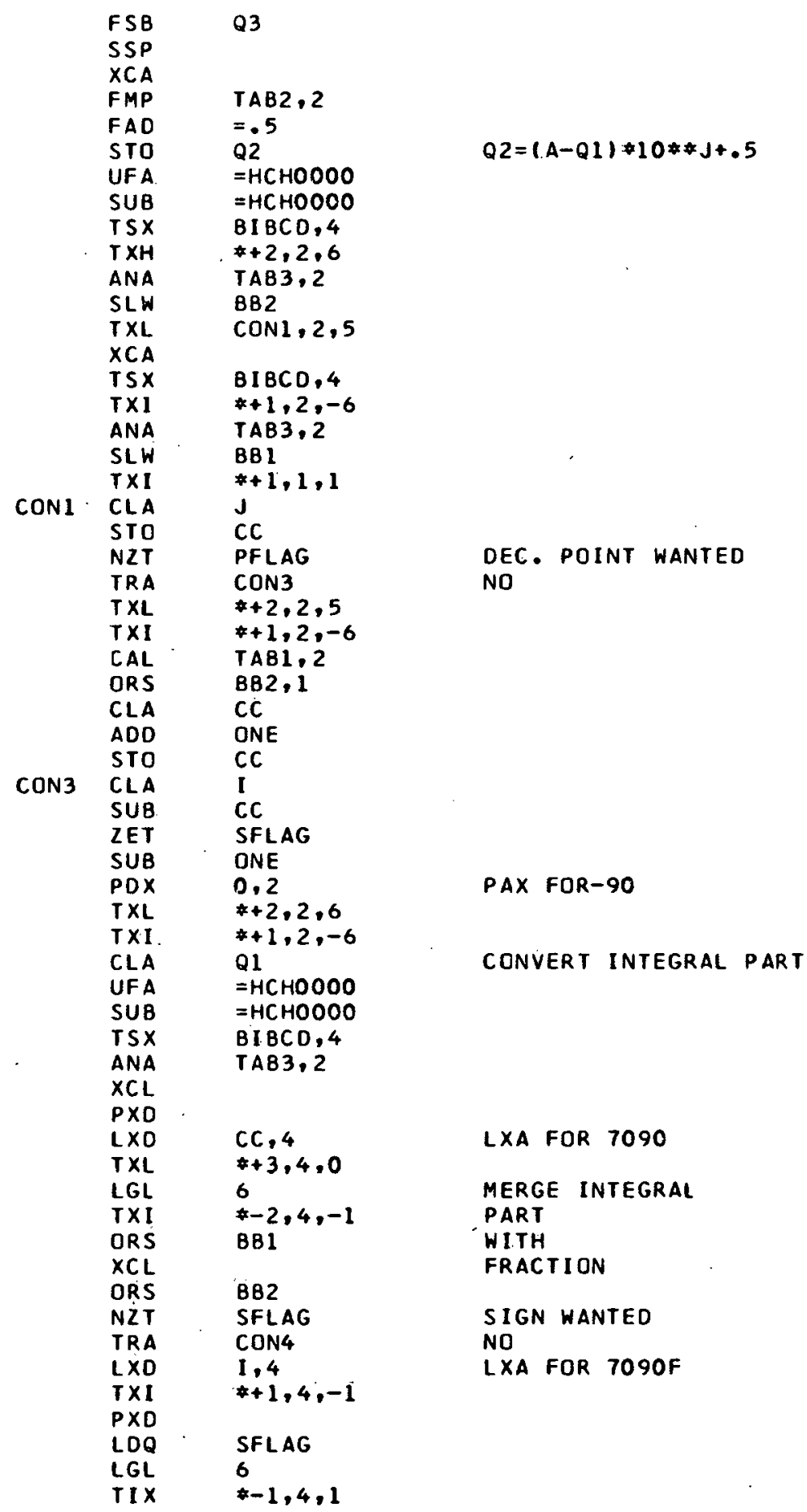

61418000

61418100

61418200

61418305

$614184 ! n$

61418500

61418600

61418700

61418800

61418900

61419000

61419100

61419300

61419400

61419500

61419600

61419700

61419800

61419900

61420000

61420100

61420200

61420400

61420500

61420600

61420700

61420800

$6142090 n$

61421000

61421100

61421200

61421300

61421400

61421500

61421600

61421700

61421800

61421900

$614220 n n$

61422100

61422200

61422300

61422400

61422500

61422600

61422700

61422800

61422900

61423000

61423100

61423200

61423300

61423400

61423500

61423600

61423700

61423800 


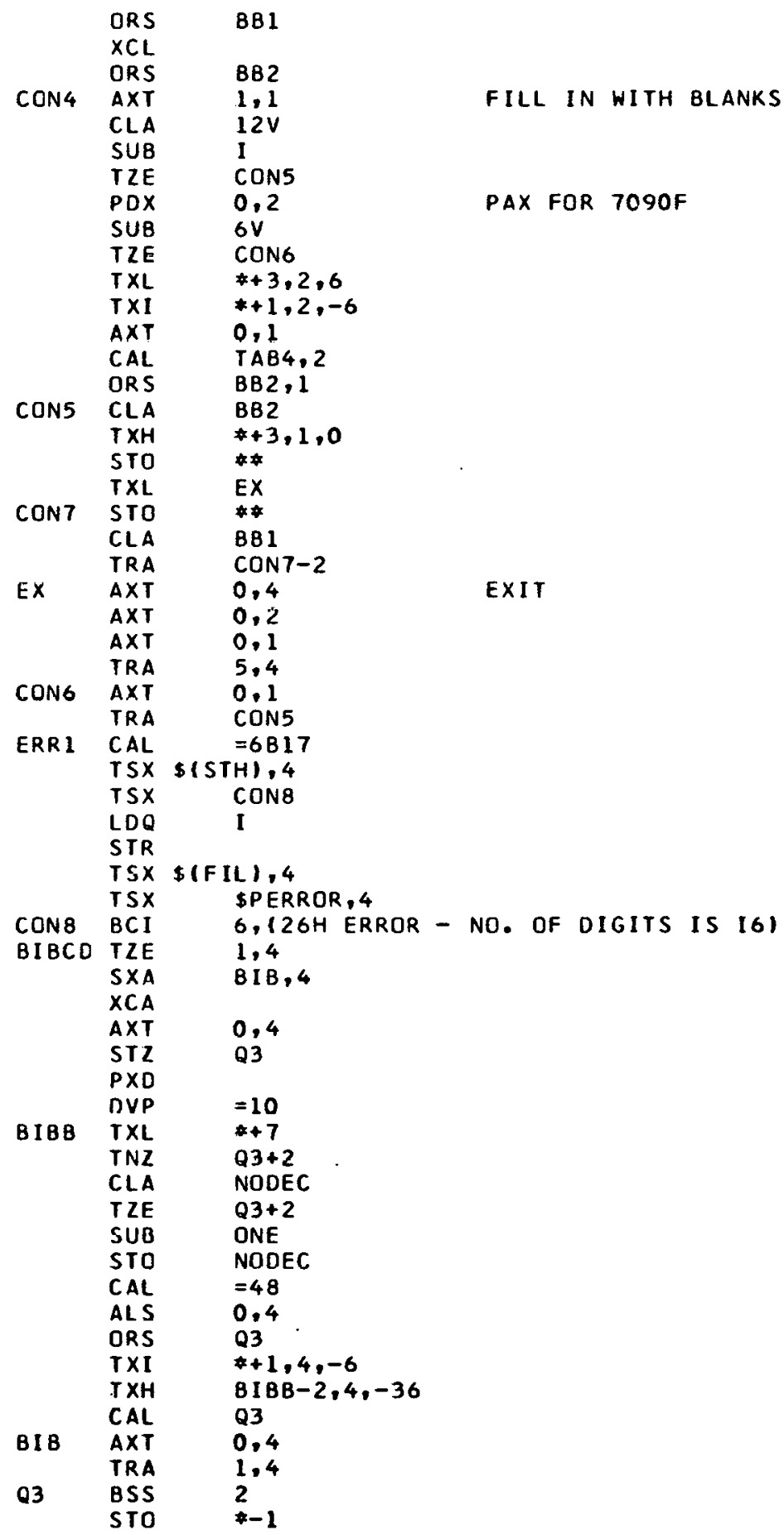

61423900

$6142400 n$

61424100

$614242 n n$

$6142430 n$

61424400

61424500

61424600

61424700

61424800

6142490 n

61425002

$6142510 n$

61425200

61425300

61425400

$614255 n$ ?

61425600

61425700

61425800

61425900

61426000

61426100

$614262 n$ ?

61426300

61426400

61426500

61426600

61426700

6142680 n

61426900

61427000

6142710 ?

61427200

61427300

61427400

61427500

61427600

$6142770 n$

61427800

61427900

61428000 $614281 \mathrm{n}$

61428300

61428400

61428500

61428600

61428700

61428800

61428900

61429000

61429100

61429200

61429300 


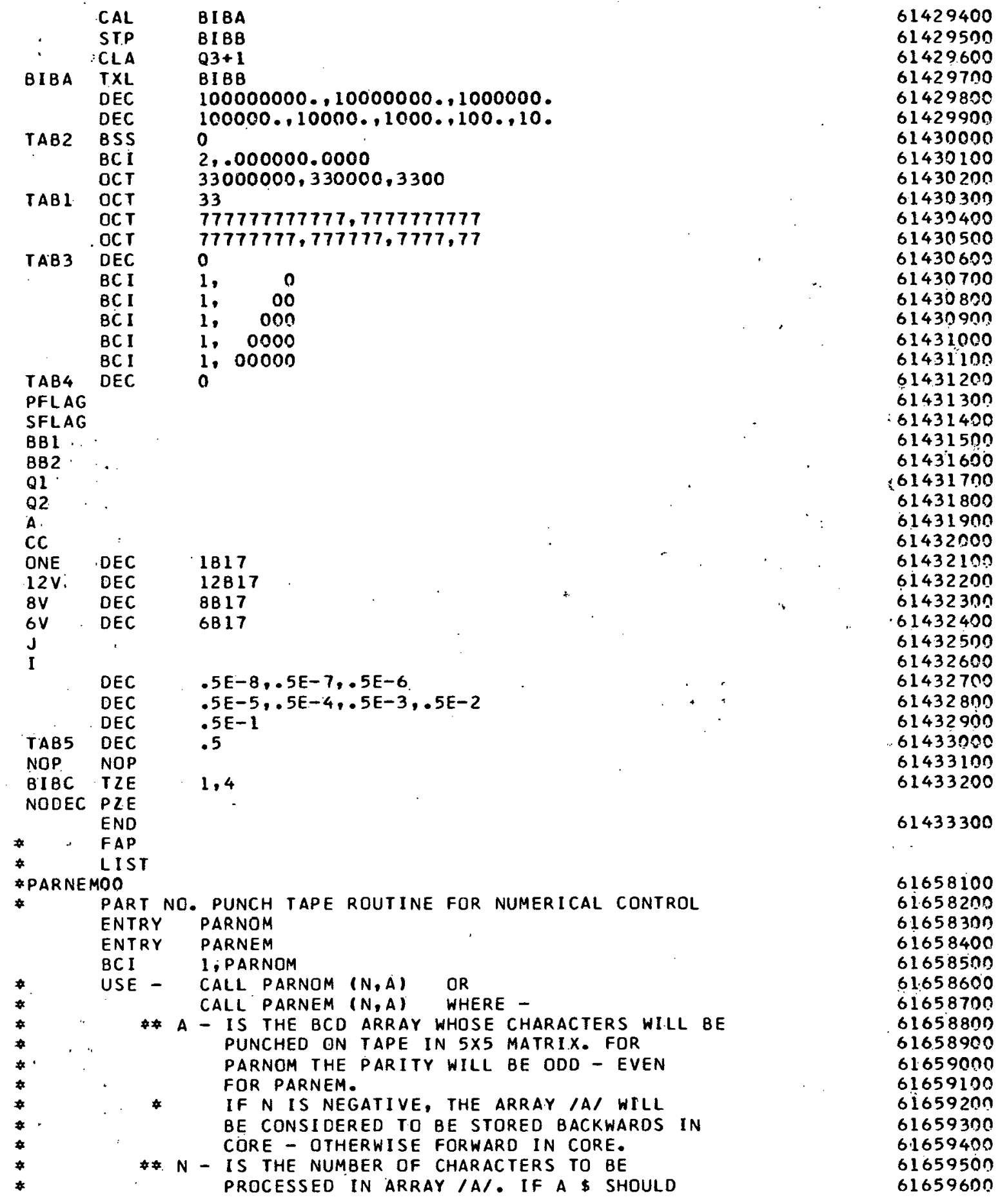


ఊ

PARNOM Cla

LDQ

$T X L$

PARNEM CLA

LDQ

STO

STQ

SXA

SXA

SXA

$S \times A$

CLA*

STO

CLA*

UFA

SUB

STO

CLA

STA

SXA

CLA *

PDX

TXI

AXT

TMI

AXC

$S \times D$

AXT

STZ

T I X

CLA

STA

PAR3 TSX

TNX

LDQ

$P \times D$

LGL

STQ

CAS

TRA

TRA

LXA

CAS

TRA

TRA

$T$ I $X$

PAR4 TIX

TXL

PAR5 PXA

SXA

ALS

PAX

F3 AXT

STI CLA

STO

ST 2

STO $50+1$

BE ENCOUNTERED, PROCESSING WILL STOP.

THIS ROUTINE SHOULD BE USED TO LABEL A PUNCHED TAPE. SO

PARNEM+2

SE

$S E+1$

ST 1

ST2

EX.4

$E X+1,2$

$E X+2,1$

F 3

\$PUNIOS

GET IO

CARON+1

\$PUNNOM

$=\mathrm{HCHDOOO}$

$=\mathrm{HCHOOOO}$

GET SEQ. NO.

CARDN

2,4

$\angle O A D+1$

LOAD

1,4

0.2

$*+1,2,1$

1,1

$++2$

1,1

$\angle O A D+2,1$

24,4

TAP $+24,4$

*-1,4,1

CS

IS

LOAD, 4

PAR 1, 2, 1

JOE

6

JOE

$=43$

$\star+2$

PAR 1

TAB 1, 4

$T A B 1.4$

$*+2$

PAR 5

* $-3,4,1$

GET SEQ. NO •

PAR $3+1,1,1$

PAR 3

0,4

F 5,1

1

0,1

0.4

$4 *, 1$

TAP, 4

$* *, 1$

IS IT

YES

SEARCH TABLE

FOUND

SET UP CHAR - COUNT

SET UP STORAGE DIR

$\mathrm{TAP}+1$

61659700

61659800

$6165990 n$

61660000

$6166 n 10 n$

61660200

61660300

61660400

61660500

$6166060 n$

61660700

61660800

61660900

61661000

61661100

6166120 ?

61661300

61661400

61661500

61661600

61661700

61661800

61661900

$616620 n 0$

61662100

61662200

61662300

61662400

61662500

$6166260 n$

61662700

61662800

61662900

61663000

$616631 \mathrm{nn}$

61663200

61663300

61663400

61663500

$6166360 n$

61663700

61663800

61663900

61664000

61664100

616642 nn

61664300

61664400

61664500

61664600

61664700

61664800

61664900

61665000

61665100

61665200

61665300

61665400

61665500 


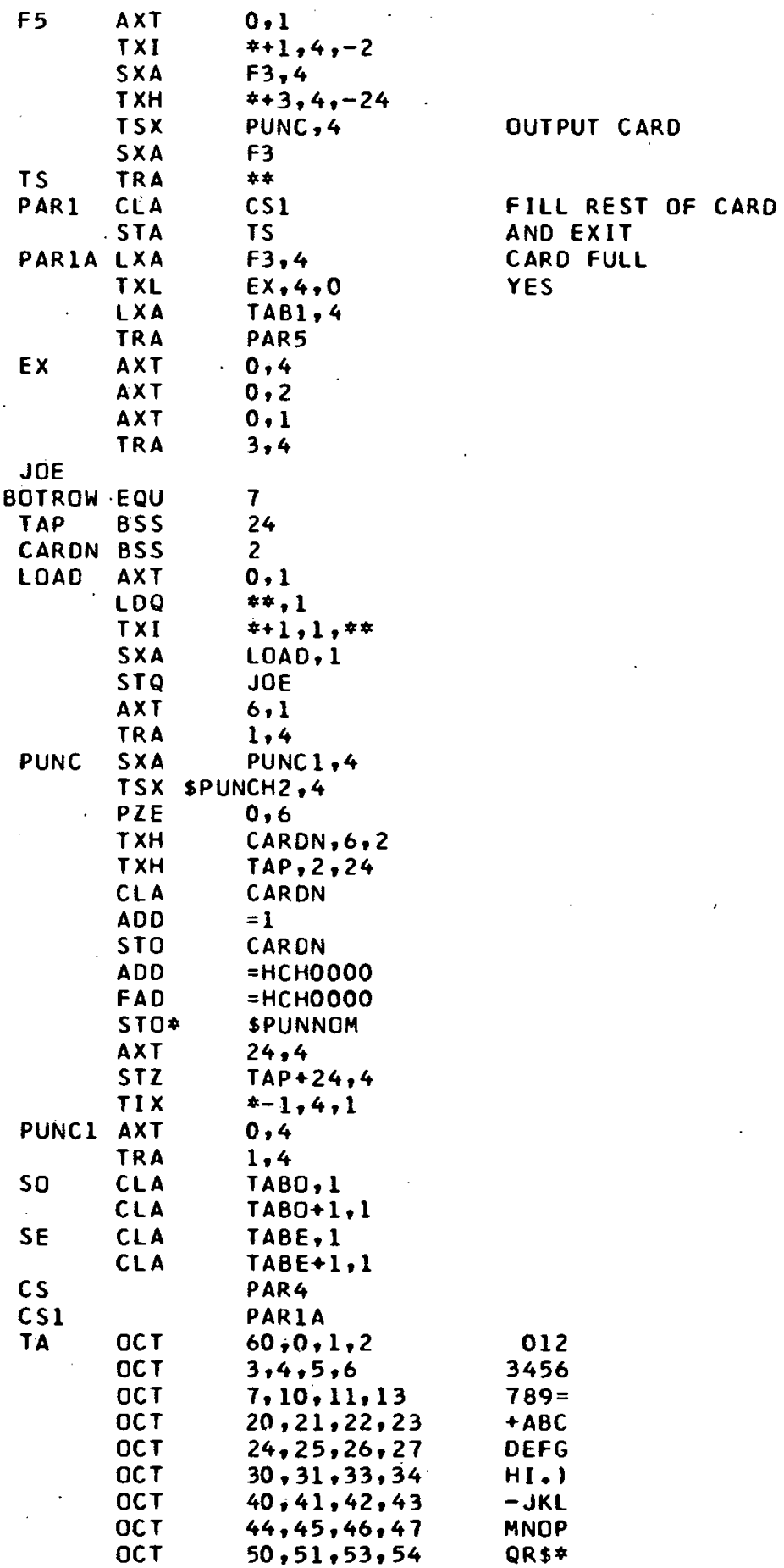

61666000

61666100

61666200

61666300

61666400

6166650 ?

61666600

61666700

61666800

61666900

61667000

61667100

61667200

61667300

61667400

61667500

61667600

61667700

61667800

61667900

$6166800 n$

61668100

61668200

61668300

61668400

61668500

61668600

61668700

61668800

61668900

61669000

61669100

61669200

$6166930 n$

61669400

61669500

61669600

61669700

616698 n

61669900

6167000 ?

61670100

616702 กn

$6167030 n$

61670400

6167050 n

61670600

61670710

61670800

61670900

61671000

61671100

61671200

$616713 n n$

61671400 


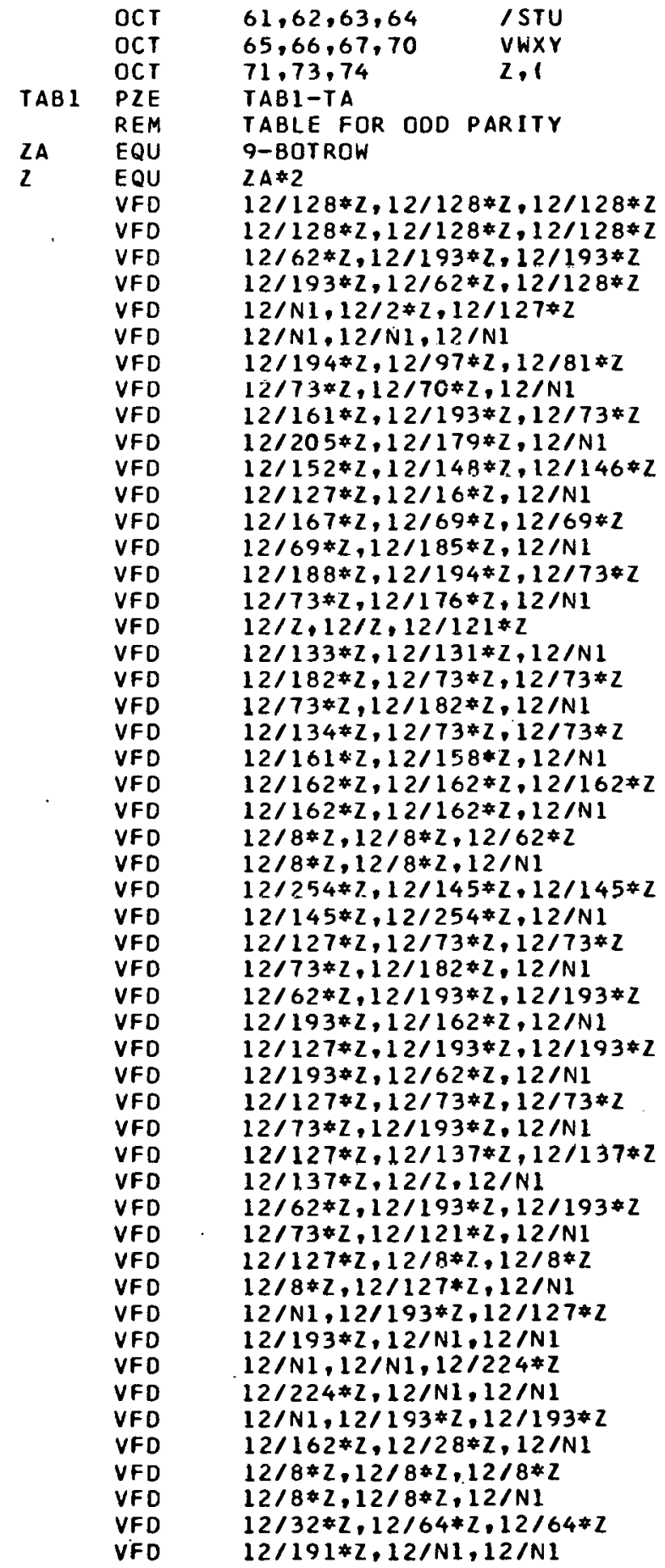

61671500 61671600 61671700 61671800 616719.20 61672000 $6167210 n$ BL61672200 BL61672300 61672400 61672500 $1616726 n n$ 161672700 $2616728 n$ ? 261672900 361673000 361673100 461673200 $4616733 n n$ 561673400 561673500 $6616736 n$ ? 661673700 761673800 761673900 861674000 861674120 961674200 $9616743 n n$ $=61674400$ $=6167450 n$ $+616746 n 0$ $+61674700$ A6167480? A61674900 B616750n0 B61675100 C61675200 C61675300 D61675400 D616755nO E61675600 E61675700 Fo1 675800 F61675900 G61676000 G61676100 H61676200 H61676300 I 61676400 161676500 .61676600 .61676700 $1616768 n 0$ 61676900 $-61677000$ $-61677100$ $J 61677200$ j61677300 
K61677400 K61677500 L61677600 L61677700 61677800 61677900 N6167800n N61678100 061678200 061678300 P61678400 P61678500 Q61678600 Q61678700 R6167880n R61678900 $\$ 61679000$ $\$ 6167910$ 2 *61679200 *61679300 161679400 161679500 S61679600 561679700 T61679800 T61679900 U6168000응 U61680100 V61680200 V61680300 W61680400 W6168n50n $\times 61680600$ $\times 61680700$ Y 61680800 Y61680900 261681000 261681100 .61681200 .61681300 61681400 161681500 6168160 ? 61681700 61681800 61681900 BL6168200n BL61682100 $616822 n 0$ 61682300 $16168240 n$ 16168250 n 261682600 261682700 361682800 361682900 461683000 $46168310 \mathrm{C}$ 56168320 n 
A6168460D

$A 61684700$

B61684800

B61684900

C.61685000

C61685100

61685200

061685300

6168540 n

E61685500

F6168560n

F6168570n

G61685800

G61685900

H61686000

H616861no

I 61686200

161686300

.61686400

.61686500

1616866 nn

161686700

-6168680 n

$-61686900$

J61687000

J616871nn

K61687200

K6168730n

L616874n?

L61687500

61687600

6168770 ?

N61687800

N616879n!

061688000

061688100

P616882nก

P61688300

Q61688400

061688500

R6168860n

R6168870n

$\$ 61688800$

$\$ 61688900$

*61689000

*61689.10n 


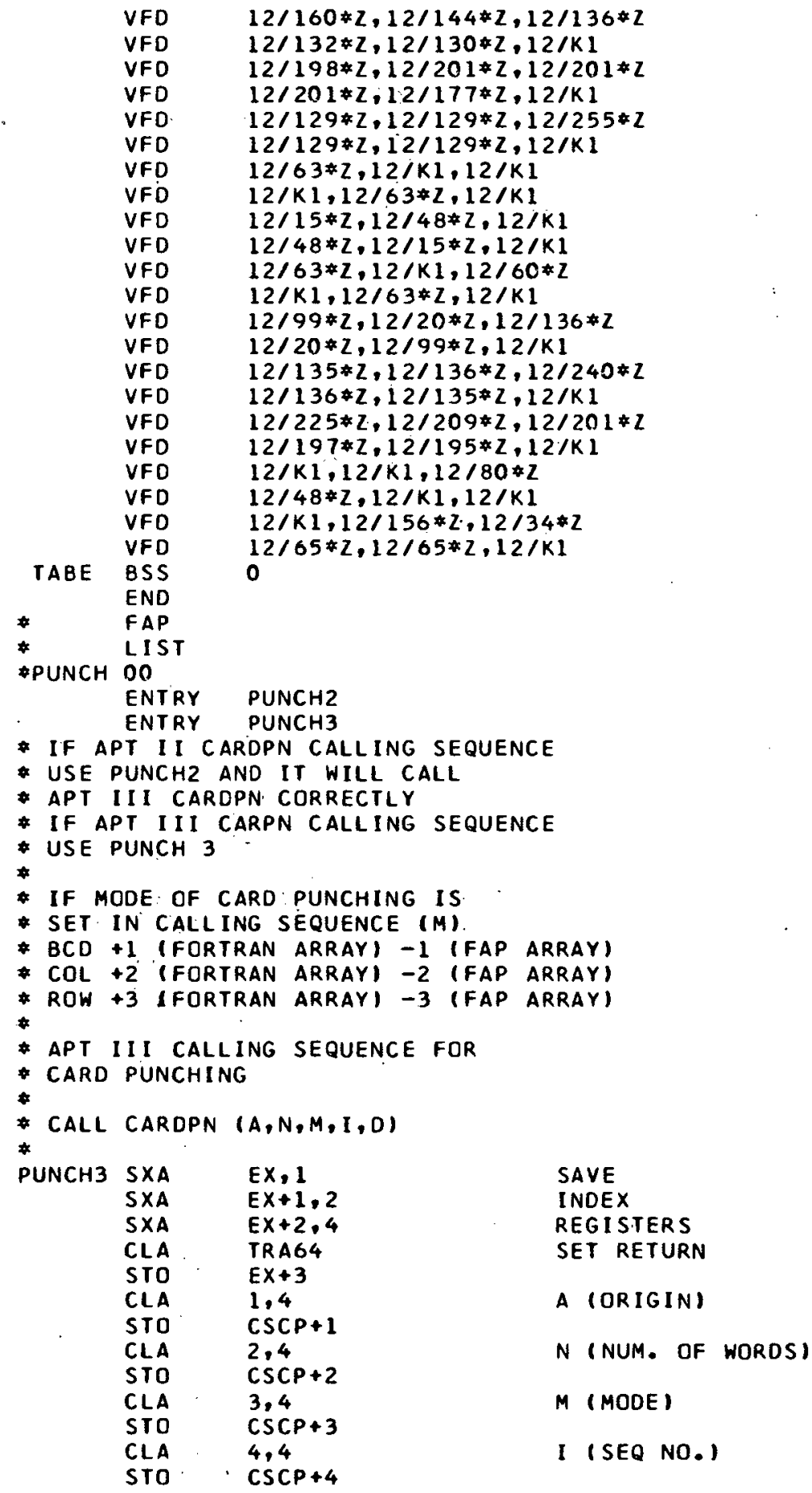

161689200 $16168930 n$ 561689400 S61689500 T61689600 T616897nn U61689800 U61689900 v616900nn V61690100 W6 1690200 W6169030D $\times 61690400$ $\times 61690500$ 61690600 Y6169070ก 261690800 261690900 .61691000 .61691100 $6169120 n$ 161691300 $616914 n n$ 61691500

61749800 61749900 61750000 61750100 61750200 $6175030 n$ 61750400 61750500 61750600 61750700 61750800 61750900 $6175100 n$ 61751100 61751209 61751300 $6175140 n$ 61751500 61751600 61751700 61751800 61751900 61752000 61752100 61752200 61752300 61752400 61752500 $617526 n 0$ 61752700 61752800 61752900 61753000 


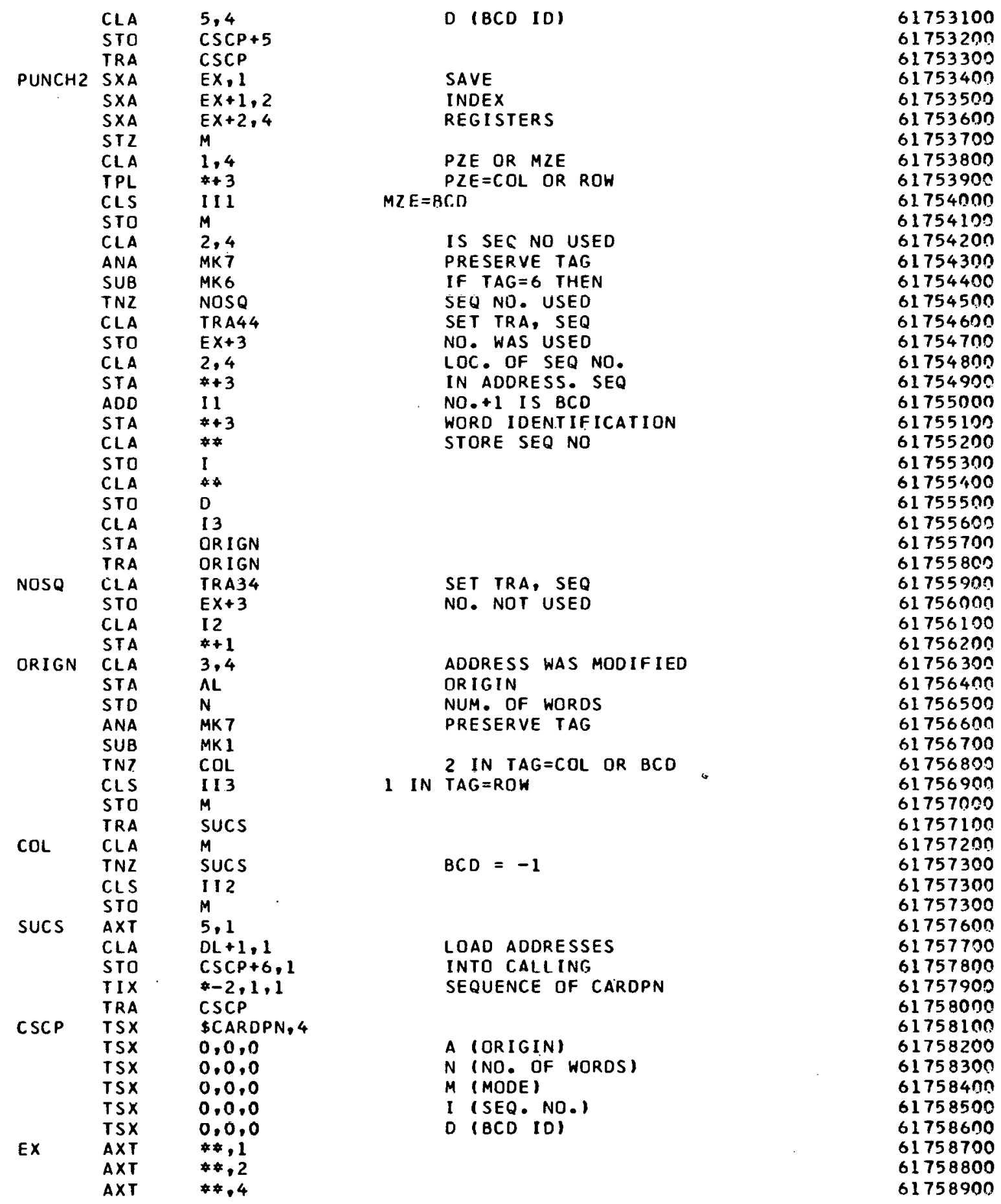




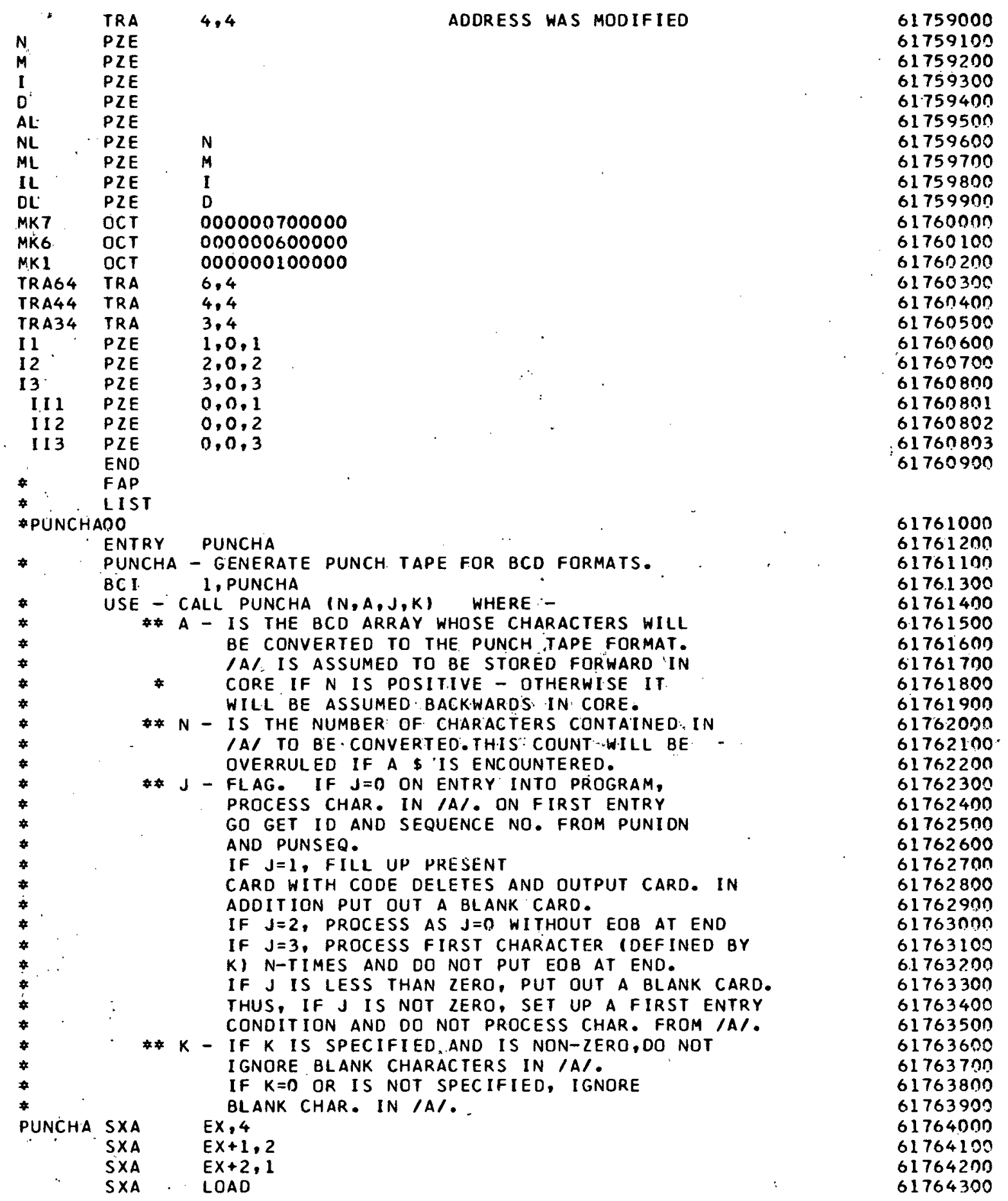




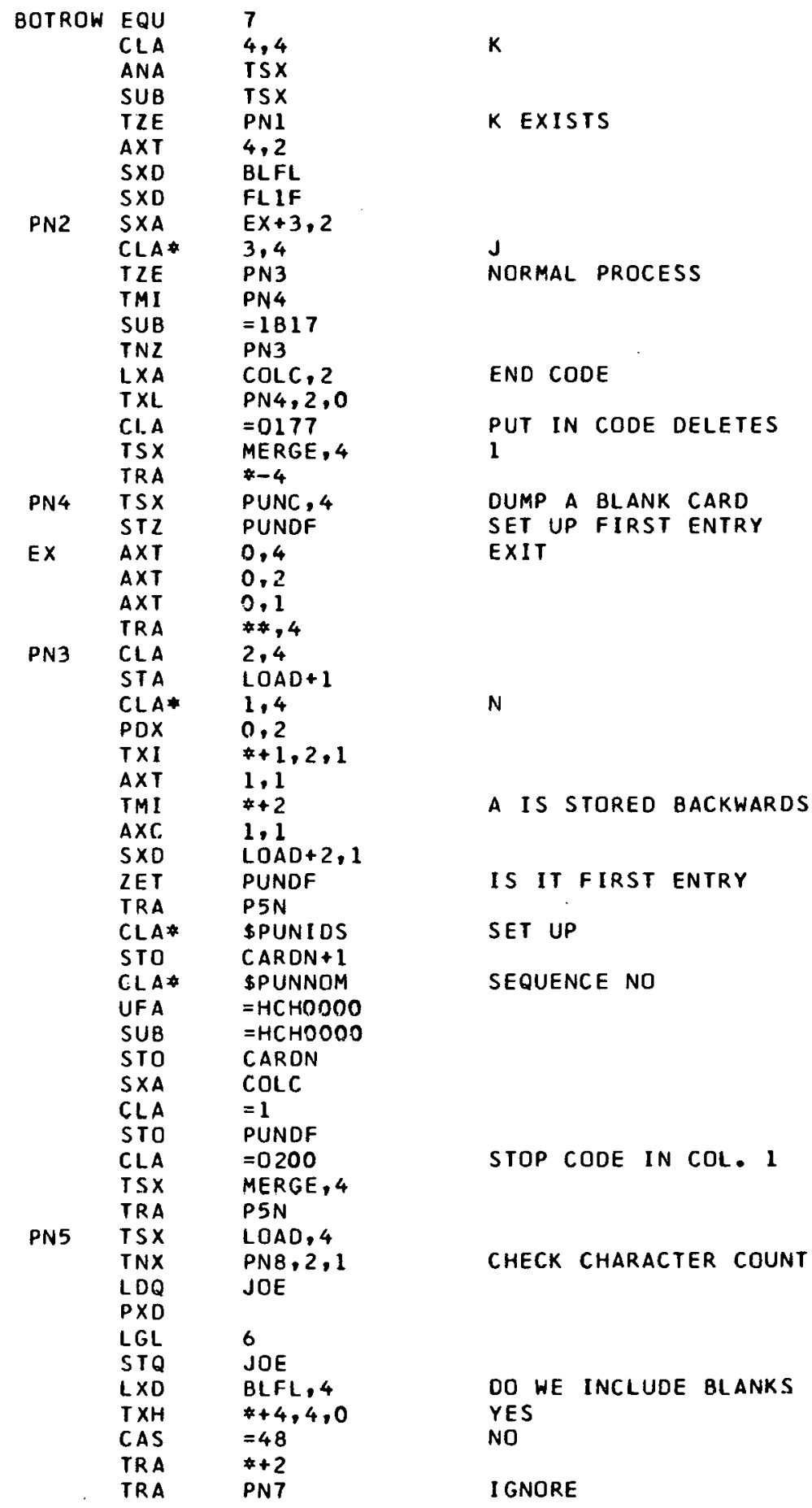

61764400

61764500

61764600

61764700

61764800

6176490 ?

61765000

61765100

61765200

61765300

617654 n!

61765500

61765600

61765700

61765800

61765900

61766000

61766100

61766200

61766300

61766400

61766500

61766000

61766700

617668 n

61766900

$6176700 n$

61767100

$617672 n 0$

61767300

61767400

$617675 n$

61767600

$6176770 n$

61767800

61767900

61768000

61768100

61768230

61768300

61768400

61768500

61768600

61768700

61768800

61768900

61769000

61769100

61769200

61769300

61769420

61769500

61769600

61769700

61769800

61769900

61770000

61770100

$6177020 n$ 


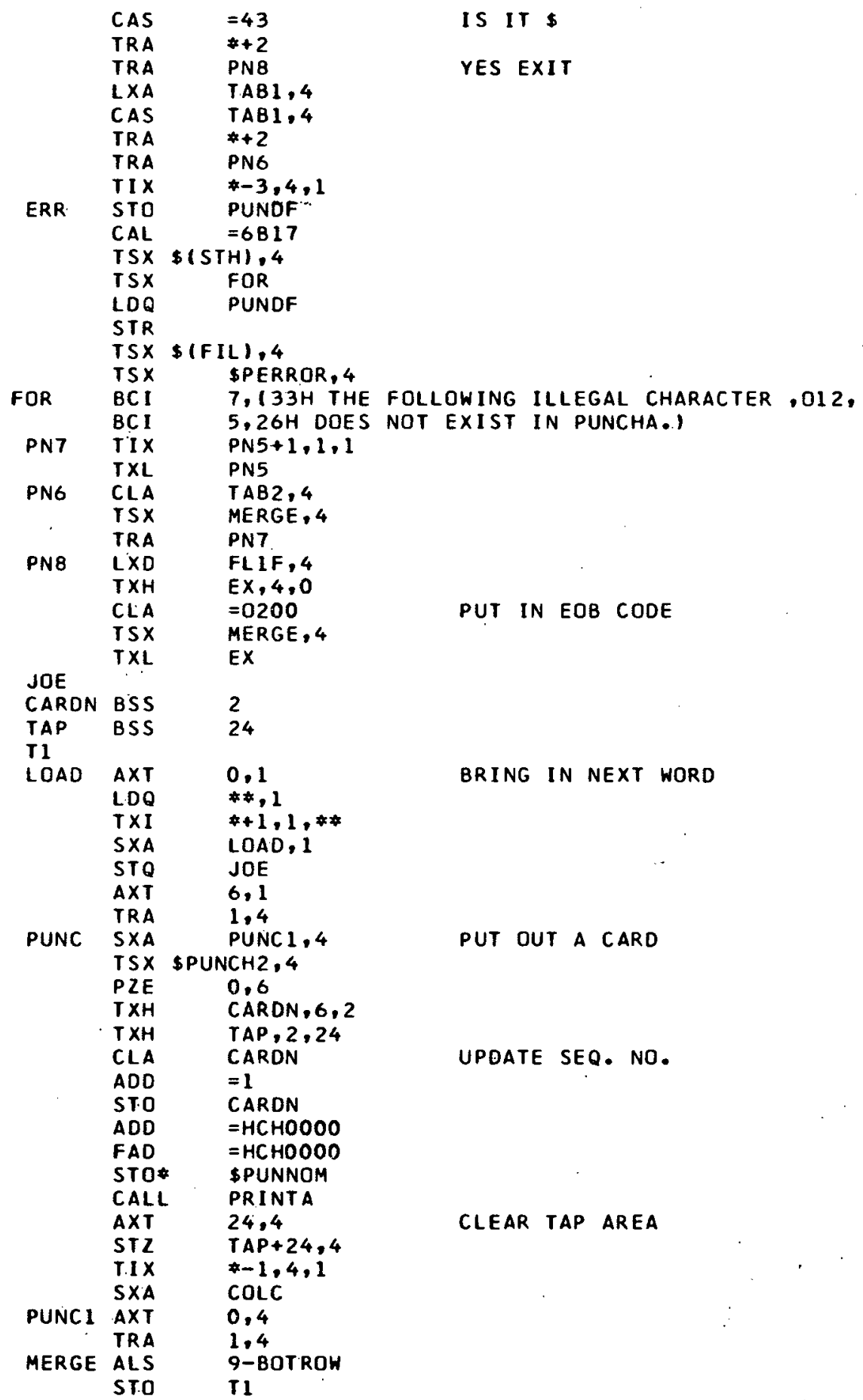

61770300

61770400

61770500

61770600

61770700

61770800

61770900

61771000

61771100

61771200

61771300

61771400

61771500

61771600

$6177170 n$

61771800

61771900

61772000

$6177210 n$

$617722 \mathrm{nn}$

61772300

617724 ?

61772500

61772600

61772700

61772800

61772900

61773000

61773100

61773200

61773300

61773400

61773.500

61773600

61773700

61773800

61773900

61774000

61774100

61774200

61774300

61774400

61774500

61774600

61774700

61774800

61774900

61775000

61775100

61775200

61775300

61775400

61775500

6177560 n

61775700

61775800

61775900

61776000 


\begin{tabular}{|c|c|c|c|}
\hline & $S \times A$ & EXI, 2 & \\
\hline & $S \times A$ & $E X I+1,4$ & \\
\hline $\operatorname{COLC}$ & $A \times T$ & 0,4 & COL. COUNT IN $\times 4$ \\
\hline & $\mathrm{TXH}$ & $M 2,4,71$ & CHECK COUNT \\
\hline & $T \times I$ & $*+1,4,1$ & CARD NOT FULL \\
\hline & $S \times A$ & $\operatorname{COLC}, 4$ & INDEX COUNT \\
\hline & $T \times H$ & $*+4,4,1$ & \\
\hline & SXA & M3 & \\
\hline & $A \times T$ & $-24,4$ & \\
\hline & SXA & $M 4,4$ & \\
\hline M3 & $\Delta \times T$ & 0,4 & WORO INDEX \\
\hline M4 & $A \times T$ & $-24,2$ & SHIFT COUNT INDEX \\
\hline MS & ALS & 0,2 & \\
\hline & ORS & TAP, 4 & \\
\hline & TXL & $M 6,2,0$ & IS PUNCH WORD FULL \\
\hline & $T \times I$ & $*+1,2,12$ & NO - INDEX SHIFT \\
\hline & $S \times A$ & $M 4,2$ & \\
\hline M7 & LXA & $\mathrm{COLC}, 4$ & IS CARD FULL \\
\hline & $\mathrm{TXH}$ & $M 8,4,71$ & YES \\
\hline EXI & $A \times T$ & 0,2 & EXIT \\
\hline & AXT & 0,4 & \\
\hline & TRA & 1.4 & \\
\hline M8 & T S $x$ & PUNC, 4 & PUNCH OUT CARD \\
\hline & $T X L$ & $E \times I$ & \\
\hline M6 & $A \times T$ & $-24,2$ & WORD FULL \\
\hline & $S \times A$ & $M 4,2$ & \\
\hline & $T \times I$ & $*+1,4,-1$ & INDFX WחRD \\
\hline & $S \times A$ & $M 3,4$ & \\
\hline & $T \times L$ & M7 & \\
\hline M2 & TSX & PUNC, 4 & CARD FULL \\
\hline & CLA & $\mathbf{T}$ & \\
\hline & $T X L$ & $\operatorname{COLC}$ & GO PROCESS \\
\hline $\begin{array}{l}\text { ZERO } \\
\text { PUNDF }\end{array}$ & & & \\
\hline TA & $\mathrm{OCT}$ & $0,1,2,3$ & $0-3$ \\
\hline & OCT & $4,5,6,7$ & 4-7 \\
\hline & OCT & $10,11,13,20$ & $89=+$ \\
\hline & OCT & $21,22,23,24$ & $A B C D$ \\
\hline & OCT & $25,26,27,30$ & EFGH \\
\hline & OCT & $31,33,34,40$ & $1.1-$ \\
\hline & OCT & $41,42,43,44$ & JKLM \\
\hline & OCT & $45,46,47,50$ & NOPQ \\
\hline & nC.T & $51.53 .54,60$ & $R \$$ \$ \\
\hline & OCT & $61,62,63,64,65$ & I STUV \\
\hline & OCT & $66,67,70,71$ & WXYZ \\
\hline & OCT & 73,74 & .1 \\
\hline TAB 1 & PZE & TAB1-TA & \\
\hline & REM & TABLE 2 & \\
\hline & OC T & $40,1,2,23$ & $0-3$ \\
\hline & OCT & $4,25,26,7$ & $4-7$ \\
\hline & DCT & $10,31,177,160$ & $89=+$ \\
\hline & OCT & $141,142,163,144$ & $A B C D$ \\
\hline & OCT & $165,166,147,150$ & EFGH \\
\hline & OCT & $171,153,13,100$ & $1 \cdot 1-$ \\
\hline & OCT & $121,122,103,124$ & JKLM \\
\hline . & OCT & $105,106,127,130$ & NOPQ \\
\hline & OCT & $111,200,76,20$ & $R \$ *$ \\
\hline & OCT & $61,62,43,64,45$ & I STUV \\
\hline & DCT & $46,67.70,51$ & WXYZ \\
\hline
\end{tabular}

61776100 61776200

61776300

$617764 \mathrm{nn}$

61776500

61776600

61776700

61776800

61776900

61777000

617771 n

61777200

$617773 n$

61777400

61777500

$617776 \mathrm{ng}$

61777700

61777800

61777900

61778000

$617781 \mathrm{nn}$

61778200

$6177830 n$

61778400

61778500

61778600

61778700

61778800

$6177890 \%$

$6177900 n$

61779100

61779200

61779300

61779400

61779500

61779600

61779700

61779800

61779900

61780000

61780100

61780200

61780300

61780400

61782500

61780600

61780700

$617808 n n$

61780900

61781000

61781100

61781200

$6178130 n$

61781400

61781500

61781600

61781700

61781800

61781900 


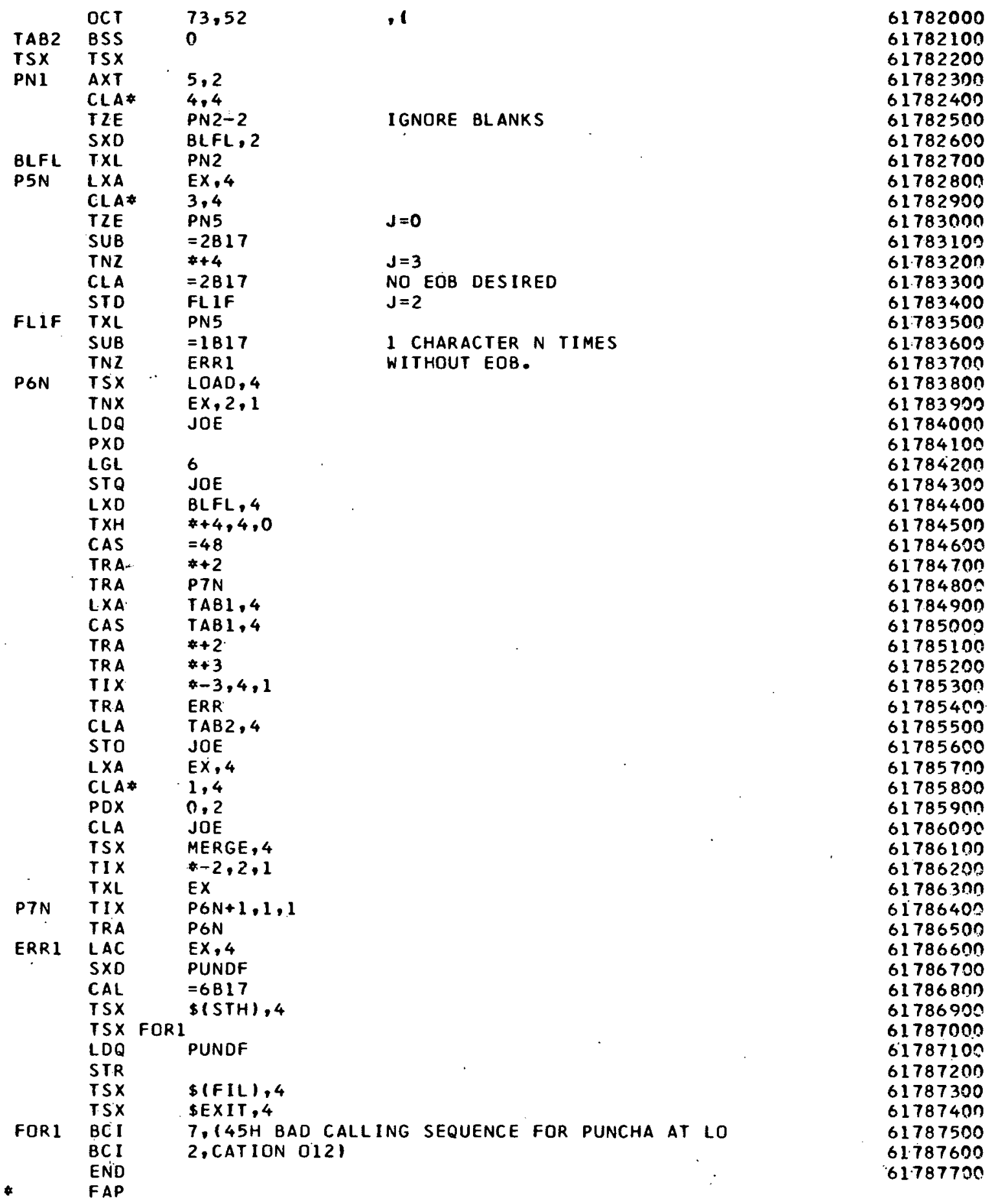


* LIST

* PUNCh CARd ID AND SEQUence nO. ROUTINe

ENTRY PUNIDN

ENTRY PUNSEQ

ENTRY PUNNUM

ENTRY PUNNOM

ENTRY PUNIDS
- PROGRAMMER USES - PUNION - TO SET UP ID

61842400

61842500

61842600

61842700

61842800

61842900

PROGRAMS USES - PUNIDS - INDIR. TO GET IO

61843000

PROGRAMMER USES - PUNSEQ - TO SET UP SEQ. NO.

61843100

* PROGRAMS UPDATES - PUNNOM - INDIR.
* PROGRAMMER INTEROGATES - PUNNUM - TO gET SEQ. NO.

61843200

61843300

61843400

CLA: $\quad 2,4$

SUB BITS

TZE P1

CLA $\quad 1,4$

TRA PZ

P1 CLA BLANKS

P2 STO PUNIDS

TRA 2,4

PUNIDS BSS 1

PUNNOM BSS 1

PUNSEQ CLA* 1,4

STO PUNNOM

TRA 2,4

PUNNUM CLA PUNNOM

STO* 1.4

TRA 2,4

BITS OCT $\quad-377777777777$

BLANKS BCI 1 ,

END

1.

00007760 LINES OF PRINT. UI.

61843410

$6184342 n$

61843430

61843440

61843450

61843460

61843600

61843700

$618438 \mathrm{nn}$

61843900

61844000

$6184410 n$

61844200

$618443 n n$

61844400

$6184441 n$

61844420

618445009977 


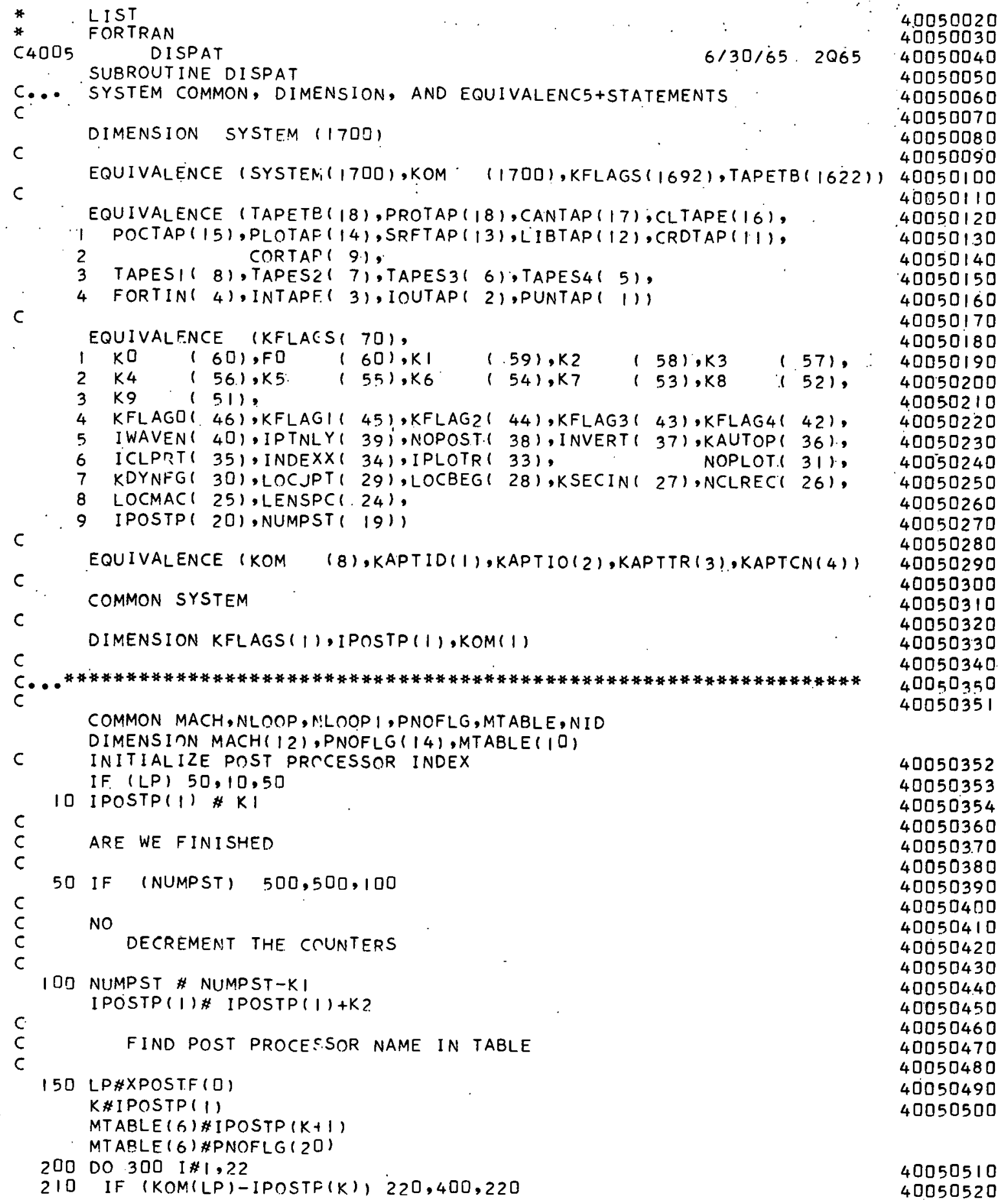




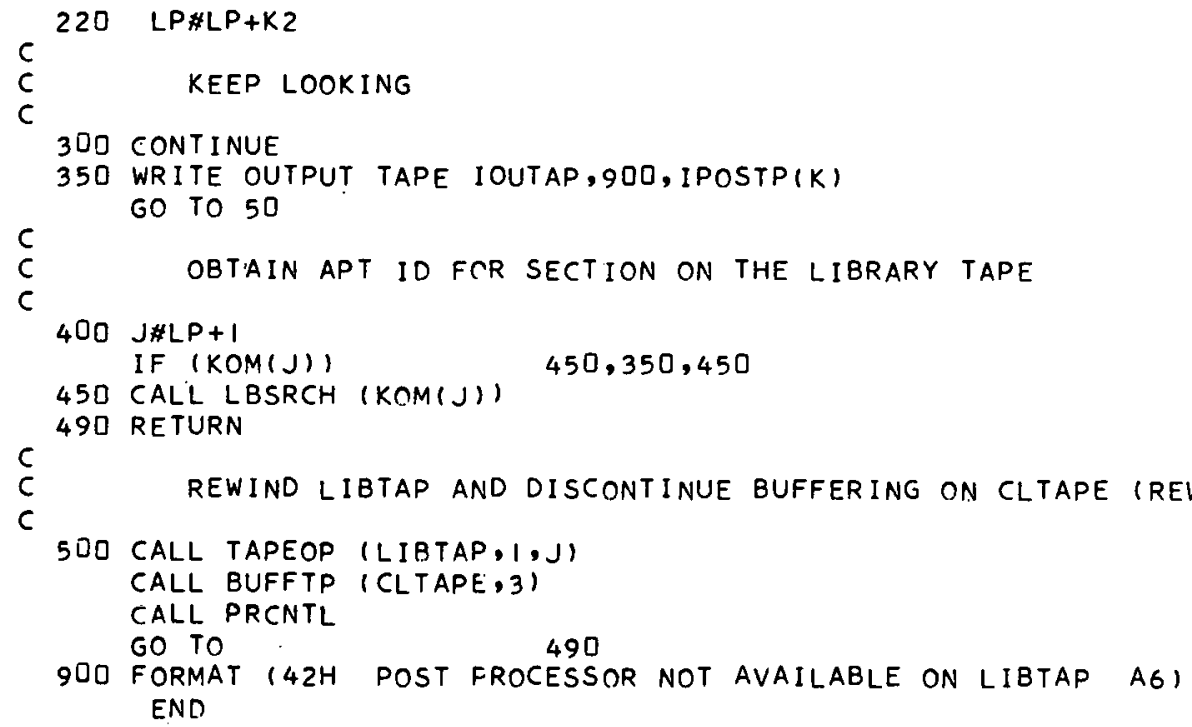

40050530 40050540

40050550

40050560

40050570

40050580

40050590

40050600

40050610

40050620

40050630

40050640

40050650

40050660

40050670

40050680

40050690

40050700

40050710

40050720

40050730

40050740

40050750 